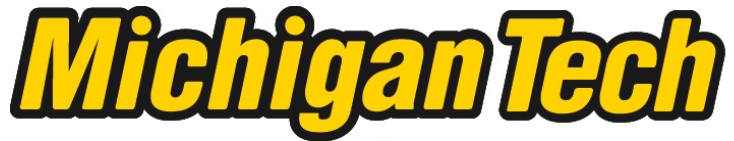 \\ Michigan Technological University Create the Future Digital Commons @ Michigan Tech
}

Dissertations, Master's Theses and Master's Reports - Open

Dissertations, Master's Theses and Master's

Reports

2011

\section{A STUDY ON THE FEASIBILITY OF UNIVERSAL CHIP CONTROL IN MACHINING}

Thimmaiah Kumbera Ganapathi

Michigan Technological University

Follow this and additional works at: https://digitalcommons.mtu.edu/etds

Part of the Mechanical Engineering Commons

Copyright 2011 Thimmaiah Kumbera Ganapathi

\section{Recommended Citation}

Kumbera Ganapathi, Thimmaiah, "A STUDY ON THE FEASIBILITY OF UNIVERSAL CHIP CONTROL IN MACHINING", Dissertation, Michigan Technological University, 2011.

https://doi.org/10.37099/mtu.dc.etds/731

Follow this and additional works at: https://digitalcommons.mtu.edu/etds

Part of the Mechanical Engineering Commons 


\title{
A STUDY ON THE FEASIBILITY OF UNIVERSAL CHIP CONTROL IN MACHINING
}

\author{
BY \\ THIMMAIAH KUMBERA GANAPATHI
}

\begin{abstract}
A DISSERTATION
Submitted in partial fulfillment of the requirements

for the degree of

DOCTOR OF PHILOSOPHY

(Mechanical Engineering - Engineering Mechanics)
\end{abstract}

MICHIGAN TECHNOLOGICAL UNIVERSITY

2011

Copyright $@$ C Thimmaiah G. Kumbera, 2011. 
This dissertation titled, "A Study on the Feasibility of Universal Chip Control in Machining", is hereby approved in partial fulfillment of the requirements for the degree of DOCTOR OF PHILOSOPHY in the field of Mechanical Engineering - Engineering Mechanics.

Department: Mechanical Engineering-Engineering Mechanics

Dissertation Advisor:

Dr. William J. Endres Date

Department Chair:

Dr. William W. Predebon Date 
Dedicated to:

My loving wife and best friend, Diya

My mother, Bollu

and,

$\mathcal{M}$ y sister, Shílpa. 


\section{ABSTRACT}

A novel solution to the long standing issue of chip entanglement and breakage in metal cutting is presented in this dissertation. Through this work, an attempt is made to achieve universal chip control in machining by using chip guidance and subsequent breakage by backward bending (tensile loading of the chip's rough top surface) to effectively control long continuous chips into small segments.

One big limitation of using chip breaker geometries in disposable carbide inserts is that the application range is limited to a narrow band depending on cutting conditions. Even within a recommended operating range, chip breakers do not function effectively as designed due to the inherent variations of the cutting process. Moreover, for a particular process, matching the chip breaker geometry with the right cutting conditions to achieve effective chip control is a very iterative process. The existence of a large variety of proprietary chip breaker designs further exacerbates the problem of easily implementing a robust and comprehensive chip control technique. To address the need for a robust and universal chip control technique, a new method is proposed in this work. By using a sin-

gle tool top form geometry coupled with a tooling system for inducing chip breaking by backward bending, the proposed method achieves comprehensive chip control over a wide range of cutting conditions.

A geometry based model is developed to predict a variable edge inclination angle that guides the chip flow to a predetermined target location. Chip kinematics for the new tool geometry is examined via photographic evidence from experimental cutting trials. Both 
qualitative and quantitative methods are used to characterize the chip kinematics. Results from the chip characterization studies indicate that the chip flow and final form show a remarkable consistency across multiple levels of workpiece and tool configurations as well as cutting conditions. A new tooling system is then designed to comprehensively break the chip by backward bending. Test results with the new tooling system prove that by utilizing the chip guidance and backward bending mechanism, long continuous chips can be more consistently broken into smaller segments that are generally deemed acceptable or good chips. It is found that the proposed tool can be applied effectively over a wider range of cutting conditions than present chip breakers thus taking possibly the first step towards achieving universal chip control in machining. 


\section{ACKNOWLEDGEMENTS}

This dissertation was the outcome of support and help of many people over the years. I would like to extend my acknowledgments all those who were responsible for my journey so far.

Foremost, I would like to thank my advisor, Dr. William Endres, for his teaching, motivation and guidance over the course of this dissertation. More importantly, Bill always urged me to discover the many mysteries of the art of machining. I also gratefully acknowledge the financial and logistical support that he extended through his business venture, Endres Machining Innovations, LLC, where this research bore fruit.

I sincerely thank my committee members, Drs. John Sutherland, Michelle Miller and Mark Johnson, for their gracious consent to guide this dissertation effort. Their comments and advice are deeply appreciated. Thanks are also due to Dr. William Predebon, Chair, ME-EM, for his continued support over the course of this work.

I would also like to express my gratitude to the ME-EM department at Michigan Tech for financially supporting me over the years. In particular, to Dr. Craig Friedrich, for ensuring I was supported through my last semester of dissertation writing. To Mike Lacourt, for his ever happy countenance and words of wisdom over my years at MTU.

Thanks are also due to my former employers, The Timken Company, for giving me a sabbatical to pursue my dream. In particular, the motivation and advice of Dr. Fukuo Hashimoto helped overcome the initial jitters of heading back to graduate school. Thanks 
also to my colleagues in the manufacturing group, who believed more in my dream than I ever imagined. Their camaraderie will be missed.

I also acknowledge the encouragement and motivation from my erstwhile advisors at UNC-Charlotte, Drs. John Patten and Harish Cherukuri.

To my many wonderful friends at MTU and in the Upper Peninsula area who helped me find my way through all the snow, I am a lucky man to have friends like you. I also thank the many practitioners of the trade who helped me learn more about the art of machining. Special mention must be made of Gary, Doug, Eric and John at EMI for the many constructive ideas and questions that helped me shape my research work.

I am forever thankful to my loving wife, Diya, who bore all my idiosyncrasies with the patience only love can bring. To my mother, Bollu, and sister, Shilpa, I owe sincere gratitude for their steadfast love and support from forever. I thank my uncle, Prof K. B. Chinappa, the former Dean of Bangalore University, for pointing me in this direction. Lastly, to my many friends and relatives, and in particular, Shree, George and Mithra, for prodding me to my last degree - cheers! 


\section{CONTENTS}

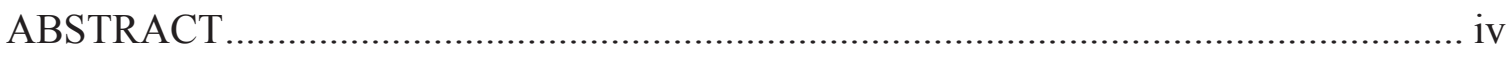

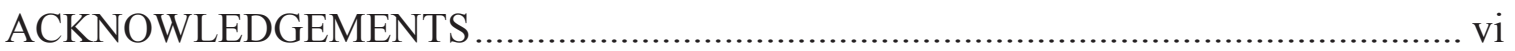

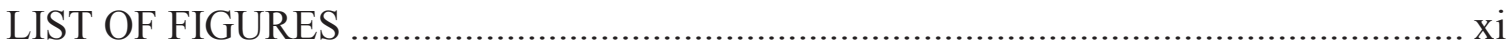

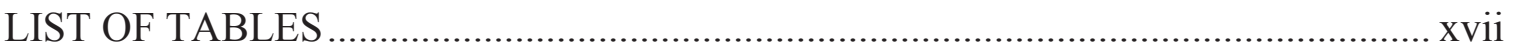

LIST OF SYMBOLS ..................................................................................

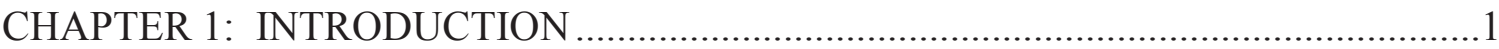

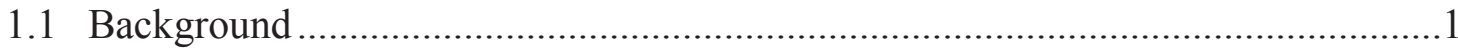

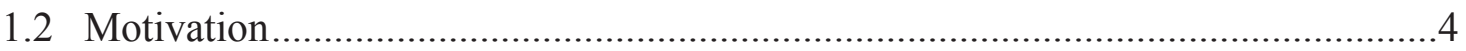

1.3 Document Overview .................................................................................

CHAPTER 2: LITERATURE REVIEW ...............................................................

2.1 Analytical Models of the Machining Process ..............................................

2.2 Chip Formation and Classification .......................................................... 15

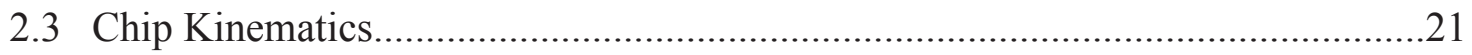

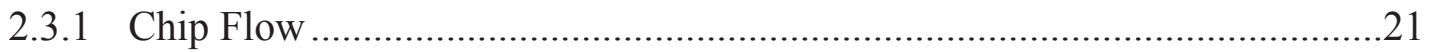

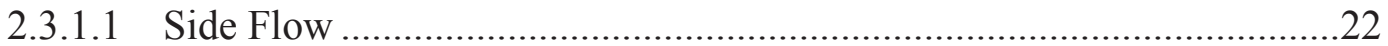

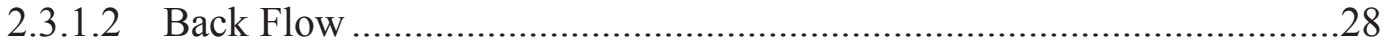

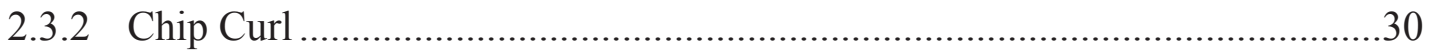

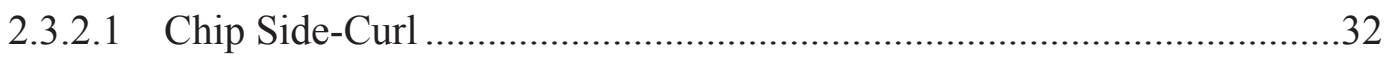

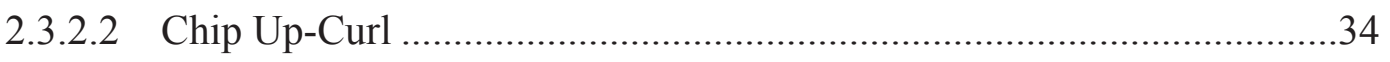

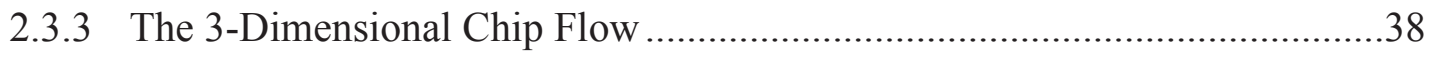

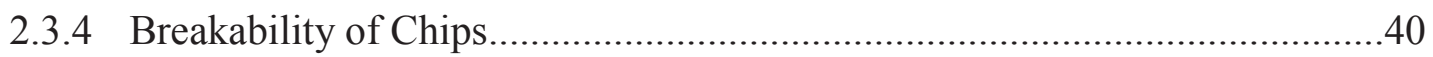

viii 


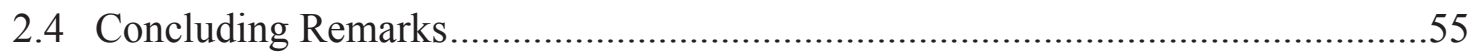

CHAPTER 3: A MODEL FOR UNIVERSAL CHIP CONTROL GEOMETRY ............56

3.1 Motivations for Universal Chip Control .............................................................56

3.2 Development of a Model for Tool Top Face Geometry ………………….............66

3.3 Defining the Inclination Angle of the Cutting Edge .............................................74

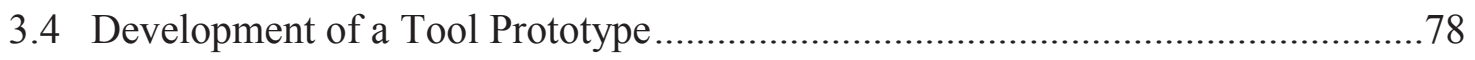

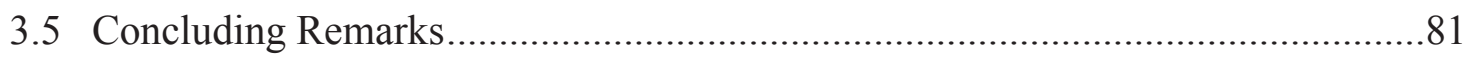

CHAPTER 4: EXPERIMENTAL STUDIES OF CHIP FLOW WITH A

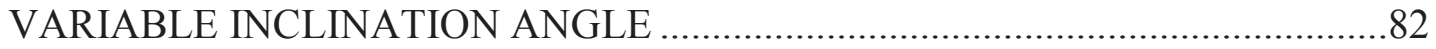

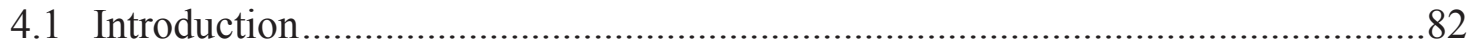

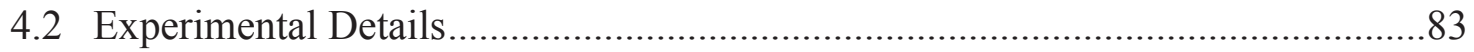

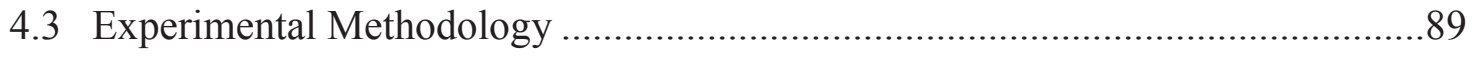

4.4 Methodology for Chip Flow Measurements ……………….................................99

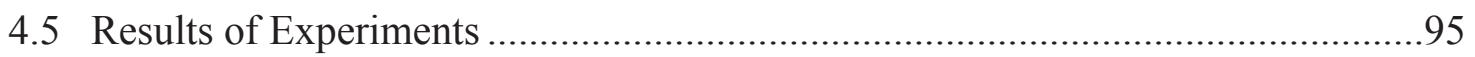

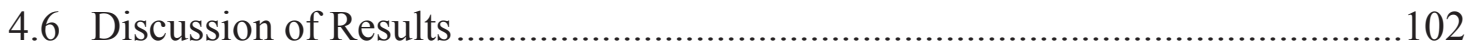

4.6.1 The Side Flow Angle and Stabler's Rule ………………............................102

4.6.2 A Qualitative Assessment of Chip Morphology ..........................................112

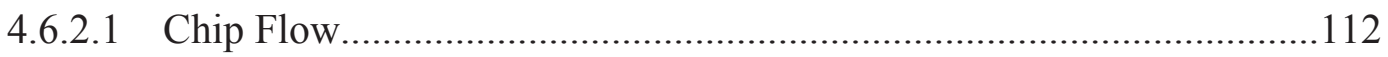

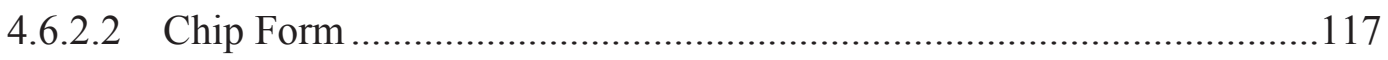

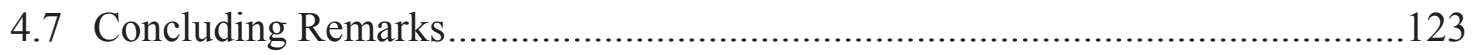

CHAPTER 5: A NEW METHOD FOR CHIP BREAKING...........................................125

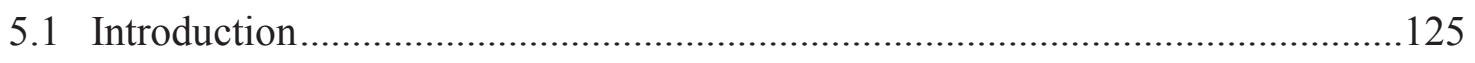

5.2 Tool System Design for Chip Breaking by Backward Bending ..........................128

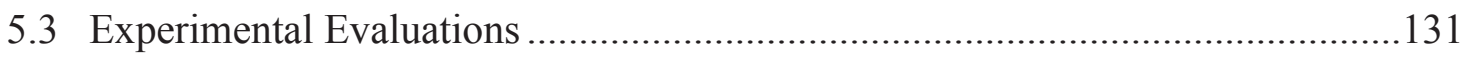

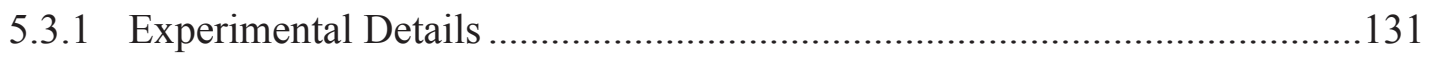


5.3.2 Experimental Results.

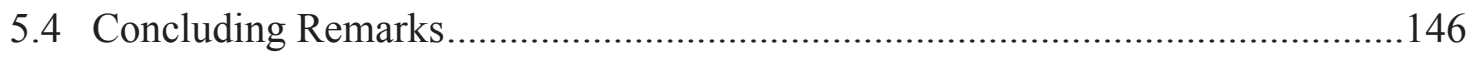

CHAPTER 6: CONCLUSIONS AND RECOMMENDATIONS FOR FUTURE

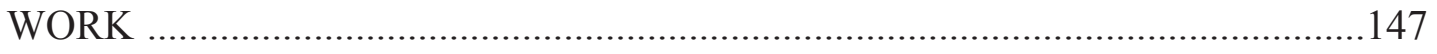

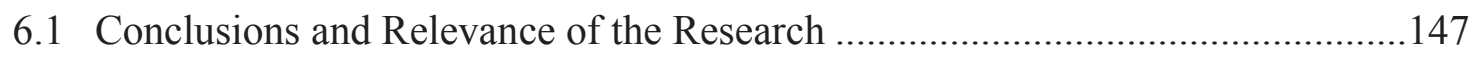

6.2 Recommendations for Future Work.......................................................... 150

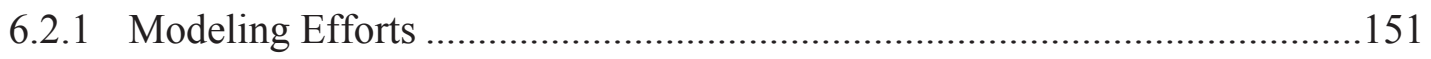

6.2.2 Further Development of the UCB Tooling System .................................151

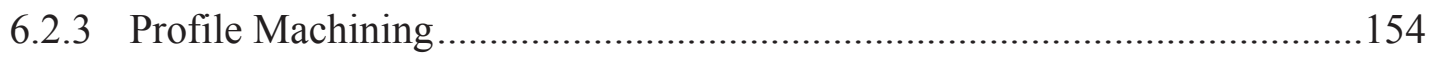

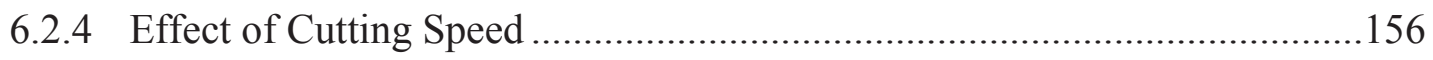

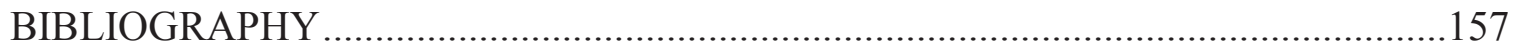

APPENDIX A: Cutting Kinematics and Tool Geometry ......................................169

APPENDIX B: Characterization of the Cutting Tool Edge........................................173

APPENDIX C: Details of Experimental Run Order............................................ 178

APPENDIX D: Measured Values of Chip Side Flow Angle...................................... 183

APPENDIX E: Replicated Results of Chip Flow Tests.........................................188

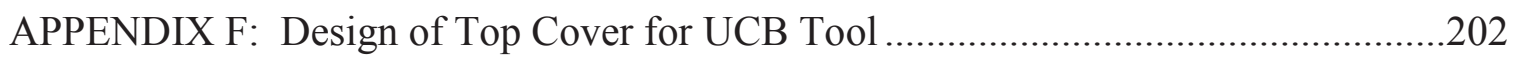

APPENDIX G: Replicated Results of Chip Breaking Tests...................................205

APPENDIX H: Replicated Results of Chip Breaking Tests with Coolant

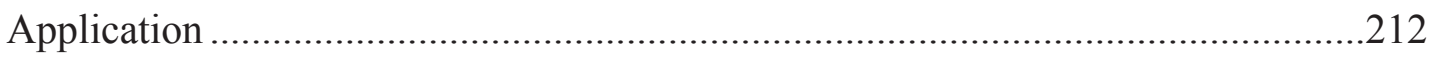

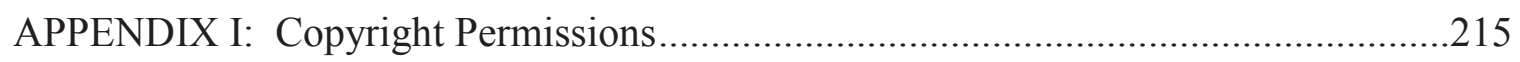




\section{LIST OF FIGURES}

Figure 2.1: (a) Orthogonal cutting model (b) Oblique cutting model (c) General $2 \mathrm{D}$ representation of the cutting process with the variables of the cutting process. .10

Figure 2.2: (a) Merchant's force considerations in machining (b) Merchant's force circle diagram showing the resultant force at the tool tip..... 11

Figure 2.3: Lee and Shaffer's slip line field model...................................................12

Figure 2.4: (a) Zorev's curved slip line model (b) The simplified version of the curved slip line model. .13

Figure 2.5: Orthogonal schema showing primary and secondary deformation zones. ...15

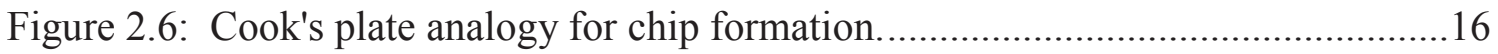

Figure 2.7: Material flow around the tool tip.................................................... 17

Figure 2.8: Piispanen's card deck model of chip formation........................................18

Figure 2.9: Four major chip morphologies (a) Type I (b) Type II (c) Type III with

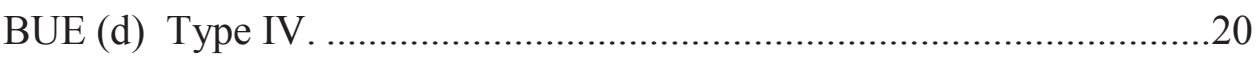

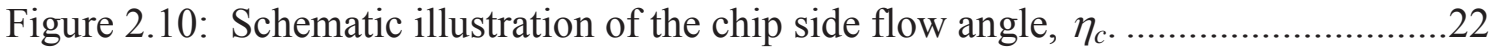

Figure 2.11: Colwell's chip flow model for (a) Sharp tool, $r_{\varepsilon}=0$ (b) Corner radiused tool with doc $>\mathrm{r}_{\varepsilon}, \mathrm{r}_{\varepsilon} \neq 0 \quad$ (c) Corner radiused tool with doc $\leq \mathrm{r}_{\varepsilon}, \mathrm{r}_{\varepsilon} \neq 0$ .24

Figure 2.12: Schematic illustration of chip back flow angle, $\eta_{b}$ (chip streaming).........28

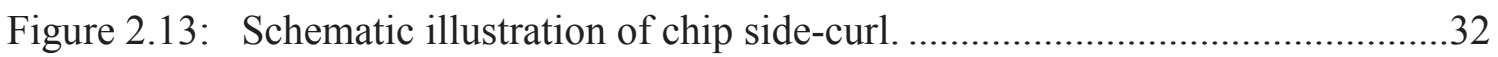

Figure 2.14: Schematic illustration of the chip up-curl............................................ 35

Figure 2.15: Up-curl radius as a function of the contact length..................................37

Figure 2.16: The 3D chip flow as (a) Individual side and up-curl components (b) Combined helix form based on the individual components.....................38

Figure 2.17: The concept of total chip control. ...................................................... 41

Figure 2.18: Nakayama's work material considerations for chip breakage. .42 
Figure 2.19: Different modes of chip breaking proposed by Nakayama .43

Figure 2.20: Chip breaker action shown for a threading operation.............................45

Figure 2.21: A sampling of chip breaker designs available.

Figure 2.22: Generalized representation of chip forms shown as a function of feed and depth of cut. .48

Figure 2.23: Kluft's recommendation for acceptable chip forms.. .............................50

Figure 2.24: Tabulated chip packing density index. .................................................51

Figure 3.1: Finnie's demonstration of chip flow changes with a minor disturbance.......57

Figure 3.2: Bending moment induced in a chip loaded under tensile and compressive loading of the top rough surface.

Figure 3.3: Schematic illustration of (a) Backward bending (b) Forward bending. ......60

Figure 3.4: Two possible cases that can result in backward bending. .60

Figure 3.5: (a)-(b) Illustration of the backward bending feasibility tests (c) Picture of mock up tooling.

Figure 3.6: Chips obtained from (left) CNMG432 benchmark insert (right) Backward bending tooling.

Figure 3.7: Change in chip forms as noted for (left) Helical coiled chip with the benchmark CNMG432 insert at a lower depth of cut and (right) Straight snarled chip with the tool design for chip backward bending without the top guidance unit. .64

Figure 3.8: Model setup showing coordinate systems and basic nomenclature. .69

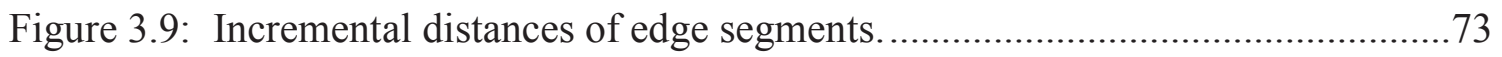

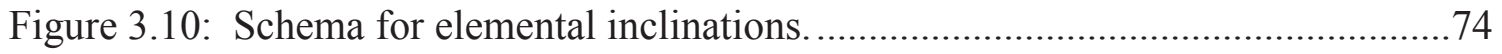

Figure 3.11: Plot of elemental inclination along tool $\mathrm{Y}-\mathrm{Z}$ axis..................................76

Figure 3.12: Insert edge geometry predicted by the model......................................77

Figure 3.13: Rendering of a S433 type prototype tool based on model generated

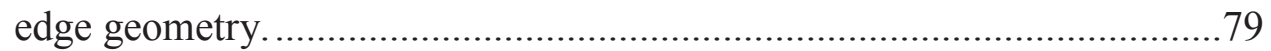

Figure 3.14: Machined prototype of a S433 type tool geometry .................................80

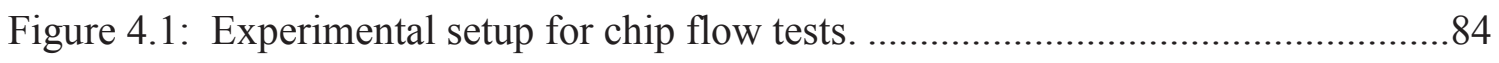

Figure 4.2: Cutting inserts and chip breakers used in the cutting test (left) Sandvik SCGT-433-PM (right) Korloy SCGT-432-AK. .88 
Figure 4.3: Methodology for chip flow measurements.

Figure 4.4: Measured chip side flow angles for: (a)-(c) Baseline tools (d)-(f) UCB tool; Work material: AISI 1018, $\psi_{r}=45^{\circ}$.

Figure 4.5: Measured chip side flow angles for: (a)-(c) Baseline tools (d)-(f) UCB

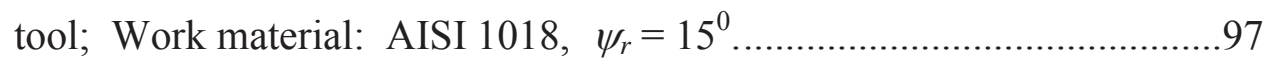

Figure 4.6: Measured chip side flow angles for AL 2024; $\psi_{r}=45^{\circ}$. 100

Figure 4.7: Measured chip side flow angles for AL 2024; $\psi_{r}=15^{0}$.

Figure 4.8: Tool-chip contact regions with variation in cutting parameters.

Figure 4.9: Photograph showing extension of tool-chip contact zone onto minor cutting edge for UCB tool. Work material: AISI 1018; $\psi_{r}=15^{0} \ldots \ldots .104$

Figure 4.10: Deviations of Stabler's rule. .106

Figure 4.11: The equivalent cutting edge for oblique cutting.

Figure 4.12: Transformed chip side flow angles using the equivalent cutting edge method; Work material: AISI 1018, $\psi_{r}=45^{\circ}, f=0.005$ ipr.

Figure 4.13: Seethaler and Yellowley's numerical model showing deviations of Stabler's rule due to friction.

Figure 4.14: Typical tool-chip contact surface for UCB tool; Work material $=$ AL 2024, $\psi_{r}=45^{\circ}, d=0.100$ inch, $f=0.012 \mathrm{ipr}$.

Figure 4.15: Photograph showing unchanged chip flow angle with rake face eliminated; Work material $=\mathrm{AL} 2024, \psi_{r}=45^{\circ}, d=0.100$ inch, $f=$ $0.012 \mathrm{ipr}$.

Figure 4.16: Chip flow patterns for: (top) Sandvik SCGT-433 insert (bottom) UCB tool; Work material $=$ AISI 1018, $\psi_{r}=45^{\circ}$.

Figure 4.17: Chip flow patterns for: (top) Sandvik SCGT-433 insert (bottom) UCB tool; Work material $=$ AISI 1018, $\psi_{r}=15^{0}$.

Figure 4.18: Chip morphology for: (top) Sandvik SCGT-433 insert (bottom) UCB tool. Work material $=$ AISI 1018, $\psi_{r}=45^{\circ}$. 118

Figure 4.19: Chip morphology for: (top) Sandvik SCGT-433 insert (bottom) UCB tool. Work material $=$ AISI 1018, $\psi_{r}=15^{0}$. 
Figure 4.20: Chip morphology for: (top) Korloy SCGT-432 insert (bottom) UCB tool. Work material $=\mathrm{AL} \mathrm{2024,} \psi_{r}=45^{\circ}$.

Figure 4.21: Chip morphology for: (top) Korloy SCGT-432 insert (bottom) UCB tool. Work material $=\mathrm{AL} \mathrm{2024,} \psi_{r}=15^{0}$.

Figure 5.1: Chip form classification based on the ISO3685:1993 standard.

Figure 5.2: Components and design of the tooling system to achieve chip breakage by backward bending.

Figure 5.3: Chip charts for: (top) UCB tool (bottom) UCB-R tool; Work material: AISI 1018, $\psi_{r}=45^{0}$

Figure 5.4: Chip charts for: (top) UCB tool (bottom) UCB-R tool; Work material:

$$
\text { AL 2024, } \psi_{r}=45^{0} \text {. }
$$

Figure 5.5: Edge build-up from dry cutting using the UCB tool. Work material:

$$
\text { AISI 1018, } \psi_{r}=45^{\circ} \text {. }
$$

Figure 5.6: Increased chip breaking region due to coolant application in the UCB

$$
\text { tool. Work material: AL 2024, } \psi_{r}=45^{\circ} \text {. }
$$

Figure 5.7: Increased chip breaking region due to coolant application in the UCB-R tool. Work material: AL 2024, $\psi_{r}=45^{\circ}$.

Figure 5.8: Chip flattening as a result of chip wedging between tool rake face and top cover. Work material: AISI 1018, $\psi_{r}=45^{0}$

Figure 5.9: (a) Increase in chip breaking region for different tool geometries (b)

Schematic explaining the reason for increase in chip breaking region in different tool geometries.

Figure 5.10: Chip forms for lower top cover channel profile; Work material: AL

$$
2024, \psi_{r}=45^{0} \text {. }
$$

Figure 5.11: Chip chart showing the apparent shift of breaking regions under lower top cover channel; Work material: AL 2024, $\psi_{r}=45^{\circ}$.

Figure 6.1: Extreme inclinations angle for a V-type insert geometry.

Figure 6.2: Schematic of the profile with a potential for possible loss of chip control.

Figure A.1: Tool geometry nomenclature 170 xiv 
Figure A.2: 3D representation of (a) Orthogonal cutting (b) Oblique cutting. 171

Figure A.3: Visualization of the inclination angle.

Figure A.4: Illustrative definition of (left) Free cutting (right) Non-free cutting.

Figure B.1: Method for photographic calibration (a) Horizontal direction (b)

Vertical direction.

Figure B.2: Tool corner radius measurement for (a) Flat tool with S-433

configuration (b) Sandvik S-433 insert (c) UCB tool with S-433

configuration.

Figure B.3: Tool corner radius measurement for (a) Flat tool with S-432

configuration (b) Korloy S-432 insert (c) UCB tool with S-432

configuration.

Figure B.4: (top) Characterization of the edge inclination with (bottom) Schematic of the direction of viewing.

Figure E.1: Chip flow patterns for Flat tool (Previous page top) Replication \#1 (

Previous page bottom) Replication \# 2 (This page top) Replication

\#3; Work material: AISI 1018, $\psi_{r}=45^{0}$.

Figure E.2: Chip flow patterns for Sandvik S-433 insert (top) Replication \#2

(bottom) Replication \#3; Work material: AISI 1018, $\psi_{r}=45^{\circ}$.....

Figure E.3: Chip flow patterns for UCB tool (top) Replication \#2 (bottom)

Replication \# 3; Work material: AISI 1018, $\psi_{r}=45^{\circ}$

Figure E.4: Chip flow patterns for Flat tool (top) Replication \#1 (bottom)

Replication \#2; Work material: AISI 1018, $\psi_{r}=15^{\circ}$. NOTE: No

data available for Replication \#3 due to tool failure.

Figure E.5: Chip flow patterns for Sandvik S-433 insert (top) Replication \#2

(bottom) Replication \# 3; Work material: AISI 1018, $\psi_{r}=15^{0}$.

Figure E.6: Chip flow patterns for UCB tool (top) Replication \#2 (bottom)

Replication \# 3; Work material: AISI 1018, $\psi_{r}=15^{0}$.

Figure E.7: Chip flow patterns for: (top) Replication \#1 - Korloy S-432 (bottom)

Replication \#1 - UCB tool; Work material: AL 2024, $\psi_{r}=45^{\circ}$. 196 
Figure E.8: Chip flow patterns for: (top) Replication \#2 - Korloy S-432 (bottom)

Replication \#2 - UCB tool; Work material: AL 2024, $\psi_{r}=45^{\circ}$.

Figure E.9: Chip flow patterns for: (top) Replication \#3 - Korloy S-432 (bottom)

Replication \#3 - UCB tool; Work material: AL 2024, $\psi_{r}=45^{\circ}$.

Figure E.10: Chip flow patterns for: (top) Replication \#1 - Korloy S-432 (bottom)

Replication \#1 - UCB tool; Work material: AL 2024, $\psi_{r}=15^{0}$.

Figure E.11: Chip flow patterns for: (top) Replication \#2 - Korloy S-432 (bottom)

Replication \#2 - UCB tool; Work material: AL 2024, $\psi_{r}=15^{0}$.

Figure E.12: Chip flow patterns for: (top) Replication \#3 - Korloy S-432 (bottom)

Replication \#3 - UCB tool; Work material: AL 2024, $\psi_{r}=15^{0}$.

Figure F.1: Detailed drawing of the UCB top cover.

.204

Figure G.1: Chip charts for Flat tool with the top guidance system ( Previous page top) Replication \#1 ( Previous page bottom) Replication \#2 (This page) Replication \#3; Work material: AISI 1018. .207

Figure G.2: Chip charts with the top guidance system for: (top) Replication \#2 UCB (R) (bottom) Replication \#2 - UCB tool; Work material: AISI 1018 .208

Figure G.3: Chip charts with the top guidance system for: (top) Replication \#3 UCB (R) (bottom) Replication \#3 - UCB tool; Work material: AISI 1018 .209

Figure G.4: Chip charts with the top guidance system for: (top) Replication \#2 UCB (R) (bottom) Replication \#2 - UCB tool; Work material: AL 2024 210

Figure G.5: Chip charts with the top guidance system for: (top) Replication \#3 UCB (R) (bottom) Replication \#3 - UCB tool; Work material: AL 2024 .211

Figure H.1: UCB chip breaking tests with coolant (top) Replication \#2 (bottom) Replication \#3.

Figure H.2: UCB-R chip breaking tests with coolant (top) Replication \#2 (bottom) Replication \#3. .214 


\section{LIST OF TABLES}

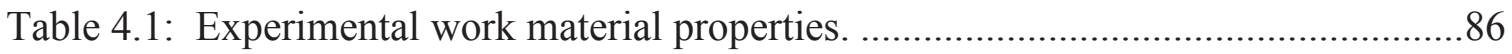

Table 4.2: Variables used in the experimental design. ...............................................90

Table 4.3: Recommended cutting parameters for baseline inserts. ..............................91

Table 5.1: Variables used in the experimental design for chip breaking tests................133

Table C.1: Run order for cutting experiments. Work material: AISI 1018, $\psi_{r}=45^{0} \ldots . .179$

Table C.2: Run order for cutting experiments. Work material: AISI 1018, $\psi_{r}=15^{0} \ldots .180$

Table C.3: Run order for cutting experiments. Work material: AL 2024, $\psi_{r}=45^{0} \ldots \ldots 181$

Table C.4: Run order for cutting experiments. Work material: AL 2024, $\psi_{r}=15^{0} \ldots \ldots .182$

Table D.1: Measured values for chip side flow angle, $\eta_{\mathrm{c}}$. Work material: AISI $1018, \psi_{r}=45^{0}$

Table D.2: Measured values for chip side flow angle, $\eta_{\mathrm{c}}$. Work material: AISI $1018, \psi_{r}=15^{0}$

Table D.3: Measured values for chip side flow angle, $\eta_{\mathrm{c}}$. Work material: AL 2024, $\psi_{r}=45^{0}$

Table D.4: Measured values for chip side flow angle, $\eta_{\mathrm{c}}$. Work material: AL 2024, $\psi_{r}=15^{0}$

Table F.1: Measurements of chip thickness for UCB tool. .203 


\section{LIST OF SYMBOLS}

$\begin{array}{ll}d & \text { Depth of cut } \\ f & \text { Feed } \\ i C & \text { Inscribed circle diameter for a cutting insert } \\ r_{i c} & \text { Radius of inscribed circle } \\ r_{\varepsilon} & \text { Tool corner radius } \\ V & \text { Cutting speed } \\ X_{i} Y_{i} Z_{i} & \text { Co-ordinates in the tool coordinate system } \\ X_{l} Y_{l} Z_{l} & \text { Co-ordinates in the tool corner coordinate system } \\ \varepsilon & \text { Included angle of insert } \\ \eta_{c} & \text { Chip side flow angle } \\ \eta_{i e f f} & \text { Effective chip side flow angle } \\ \eta_{i r e q} & \text { Required chip side flow angle } \\ \lambda & \text { Tool edge inclination angle } \\ \lambda_{i} & \text { Tool edge inclination angle for } i \text { th point along tool edge } \\ \psi_{r} & \text { Tool lead angle measured in the tool reference plane } \\ \psi_{i} & \text { Tool lead angle for } i \text { th point along tool edge }\end{array}$

xviii 


\section{CHAPTER 1}

\section{INTRODUCTION}

\subsection{Background}

Throughout much of the recorded history of the modern industrial world, manufacturing of materials or the processing of raw materials into finished goods has been the fulcrum for progress. From the industrial revolution and the first steam engines came innovations that advanced the field of manufacturing. New advanced materials, processing techniques as well as management ideas were some of the many developments in the field. Despite a proliferation of different processing techniques the basic facets of production remained the same - material removal or material forming. Between the two processes, material removal, more generally referred to as machining, retains a larger share of the production process in any finished product.

Historians (Rolt, 1965) maintain that the period of scientific innovation in manufacturing started earnestly at the turn of the century. Much before the advent of scientific studies on the subject, machining remained an exclusive preserve of skilled craftsmen long plying their trades as an art form. Many of the machines and tools used were predominantly of individual design and construction. However, the introduction of the 
steam engine by Thomas Newcomen in the early $18^{\text {th }}$ century led to the invention of the earliest version of the modern boring machine. Further development in accuracies and technology of machine tools did not occur until the development of the horizontal boring machine by John Wilkinson to manufacture steam turbines for James Watt (circa 1776).

Until the invention of the first sophisticated machine tool, the cutting tool of choice was carbon steel. But the proliferation of more advanced, powerful and robust machines coupled with the new economic requirement of producing goods faster led to the development of the High Speed Steel (HSS) tool material by F. W. Taylor, a pioneer of many a revolutionary idea at the time. With its ability to work at higher temperatures and wear resistance, HSS tools enabled cutting speeds and material removal rates to improve drastically. Taylor's work also laid the foundation for the modern scientific study of machining.

The early scientific studies that followed Taylor's work were based upon empiricisms and were time consuming and expensive. The age of empiricisms was, however, a brief interlude and machining research formally entered the modern era when Hans Ernst (1938) first published his study on tool-work material interaction. Ernst's work was pioneering because it revealed the physics behind the material removal process itself. Machining could now be understood with the use of models that sought to unravel the physics of the process. With the advent of the modeling technique and subsequent understanding of the process variables, innovations increased at a drastic pace. New and better tooling - materials and design - as well process designs were introduced. A modern account of this latter period in the history of machining is well chronicled by Eugene Merchant (Merchant, 2003) in a recent tome on the history of machining. One unique 
theme obvious in both historical accounts is that machining research was always - explicitly or implicitly - driven by a need to increase productivity or, alternately, reduce costs. The economic considerations resulted in enhanced research efforts to develop new tooling, tool substrates, coatings and work materials.

In-spite of the voluminous research in the general area of machining mechanics, studies on the exact role of the by-product of machining, the chip, is more recent in history. It is now understood and acknowledged that the chip has a far more significant role than initially presumed. Apart from providing the requisite shape to the final product, the chip also serves as an indicator of the quality of the finished product. The chip formation process, shape and behavior indicate the efficacy of the process. But in modern manufacturing, with its requirement for dramatic increases in material removal and increased interest in completely automated processes, the chip also becomes a nuisance that seeks to undermine the cutting process. If not handled effectively, the long continuous stringlike chips produced when processing free machining work materials can cause entanglement at the tool-work interface and result in significant damage to the part by scratching or even denting the finished surface. Large scale entanglement of the chip can result in costly machine downtime for clearing the chip. It can also result in tool breakage due to re-cutting of chips by the cutting tool. If not adequately contained, due to the high velocities, a far more serious consequence is the threat to operator safety.

For efficient and high quality part production in automated systems, it is important that the generated chips are disposed in a safe and economical fashion. In keeping with this requirement, significant effort has been dedicated in recent years to scientifically understand the fundamental behavior of chips. These efforts have concentrated on both 
understanding chip flow (control) as well as the mechanics for efficiently breaking them into smaller, more manageable entities (disposal). In general, the two separate processes of control and disposal are addressed through the common terminology "chip control".

\subsection{Motivation}

The basic science of chip formation, chip shapes, chip flow and their significance have all been studied extensively and quantified. But the randomness of the machining process makes the development of any "universal" predictive theoretical model a difficult task. A re-course to time consuming and expensive experimentation is then inevitable.

Insofar as research into chip control is concerned, researchers have used a variety of approaches all of which can be grouped into broad areas based on the subject matter:

(1) Mechanistic models, which take the various forces and cutting conditions into consideration;

(2) Materials and metallurgical (including thermal influences) aspects that influence the chip formation and flow;

(3) Novel tool design.

The mechanistic approach deals with the prediction or evaluation of chip morphology. By using the forces and kinematics of the process to understand and predict the chip flow behavior a priori, the task of chip control is reduced to a simple one of inducing breakage of the continuous chip at some point along its flow. But a drawback that is apparent here is that the developed chip flow theories are based on idealized assumptions of the cutting process. In reality, the actual cutting process is far from ideal with multiple influencing parameters acting in unison on the chip flow behavior. Thus it becomes an 
impossible exercise to address the complete range of cutting conditions within any theoretical model of chip flow. Under such dynamic conditions, achieving effective chip breakage is a highly random process.

The materials and metallurgical approach generally seeks to understand the machinability or the relative ease of machining a particular work material. This approach specifically addresses the chip breakage issue, or the ease thereof, rather than the actual control of the chip flow. Since product design considerations dictate the material to be used, the metallurgical approach is fairly narrow in its treatment of the chip control issue.

The final method of designing tool geometries to handle chips over a specific range of cutting conditions presents a more practical and robust technique for chip control. Its effective implementation, however, depends on the fundamental understanding of the preceding two methods. Fortunately, the maturity of both the mechanistic and materials knowledge of the chip formation process provide a sound foundation to implement the tool design approach.

Having articulated the importance of tool design for chip control, the objective question relates to the most effective tool design required for universal chip control. Chip control at present is achieved by incorporating "chip breaker geometries" on the cutting tool face so that the chip flow can be altered and subsequently broken. A cursory look at the product catalogs of some of the leading manufacturers (Sandvik, 2009a; Kennametal, 2010; SECO, 2010; Korloy, 2010) reveals that there exist numerous recommendations for selecting the right chip breaker geometries. These geometries vary between different manufacturers. Further complicating matters is the fact that even within one manufacturer's product line, there exist multiple designs that address different levels of work 
materials, feeds and depths. In summary, there exists a massive database to sift through for every conceivable machining condition. To a general practitioner such a non- unified process leads to confusion and obviously, injudicious choices without expert recommendations.

For chip control geometries to be truly "universal" and simple, it then becomes paramount that there exist a minimum number of choices and also at the same time accommodate the widest possible range of machining conditions. Apart from being economically attractive in that tooling suppliers can optimize production processes and product lines, developing such a system may also realize shorter inventories and decision times for the end user.

The stated need for universal chip control tool geometry is addressed through this research. Fundamental knowledge that is already available from mechanistic models has been utilized in developing a cutting tool geometry that produces a consistent chip flow which may be evacuated from the cutting zone using a specialized tooling system design. It is presumed that such a cutting tool will obviate the present practice of using experimentation for selecting effective chip breaker tools and will create a more productive manufacturing process.

\subsection{Document Overview}

Chapter 2 presents an introduction to the topic of chip control through a survey of existing literature. Starting with a discussion on analytical models, the content builds on each topic relevant to the subject matter of chip control itself. A brief primer on chip formation and chip behavior introduces the requisite understanding and context in which 
to present the results of the research. Present chip control techniques are reviewed and novel methods in use are also included.

In Chapter 3, the original feasibility tests and motivation leading to the development of this research are presented. The development of a mechanistic model to predict the tool top face geometry is discussed. The model serves as the building block for product realization of the requisite tool geometry. To corroborate the underlying principles of the model, some assumptions used for the development of the model as well as relevant works from past researchers are discussed.

Chapter 4 introduces the experimental work to support the model. Results from experimental work on chip flow and morphology are presented. The experimental results and inferences are discussed prima facie. Some of the influential variables and their effects are also discussed.

Chapter 5 presents a new tooling system that utilizes the model based chip flow behavior to achieve chip breakage by backward bending. Results from experimental cutting tests are presented to prove the effectiveness of the proposed tooling system to universally break chips into small segments across multiple levels of cutting conditions and work materials.

Chapter 6 sums up the main conclusions from this research. Recommendations are included for further development of the technology as well as some shortcomings that need to be addressed in the near future. 


\section{CHAPTER 2}

\section{LITERATURE REVIEW}

This chapter presents a review of pertinent work on chip control research. Starting with a brief coverage of analytical models of the cutting process, the basics of the chip formation process and chip kinematics are discussed. Some key concepts of the chip breaking mechanism and the current techniques for breaking long continuous chips are also reviewed.

\subsection{Analytical Models of the Machining Process}

Analytical modeling of the machining process facilitates the fundamental understanding of the metal cutting phenomena while simultaneously reducing the reliance on empiricisms through a large number of time consuming experiments. More importantly, it enables the estimation of important process considerations such as cutting forces and helps predict the power required to optimally perform the cutting operation. Numerous analytical models predicting the relationship between the variables involved in the cutting process have been developed over the years. Finnie (1956), and more recently Komanduri (1993), have chronicled comprehensive surveys listing such analytical models. 
In its most simplistic form, the metal cutting process is assumed to be analogous to parting the work material using a sharp chisel-edged tool. Early models on metal cutting treated the chisel cutting akin to wood machining whereby material removal from the bulk raw stock occurs by fracture ahead of the tool (Finnie, 1956). However, it is now generally accepted that the material removal process during machining of metals is due to intense localized shear deformation of the work material ahead of the tool cutting edge.

A majority of analytical models available today treat the cutting process as a simplified two-dimensional approximation called Orthogonal cutting (see APPENDIX A for a discussion on cutting kinematics). In reality most cutting processes are three dimensional and can be appropriately treated by using the Oblique cutting model wherein the cutting edge is inclined at an angle of obliquity, $\lambda$, to the direction of primary motion of the workpiece. Nevertheless, subject to valid assumptions, the simplified orthogonal representation is considered a good approximation of the oblique model. Figure 2.1 illustrates the orthogonal and oblique cutting models.

From Figure $2.1(\mathrm{c})$, the work material initially moving at velocity $V$ undergoes a plastic shearing strain and results in a chip flowing at velocity $V_{\mathrm{c}}$ after passing a finite shear region represented by the shear plane AB. Due to its importance as a measure of the energy efficiency of the cutting process, the shear plane orientation, $\phi$, is fundamental to the development of analytical models on the cutting process (Shaw, 2005). In addition to defining the state of stress at the cutting zone, the orientation of the shear plane also influences the kinematics of the cutting process.

Merchant (1944) developed the most simple and commonly used model to predict the shear plane angle. He presented a mathematical concept for force relationships of the 


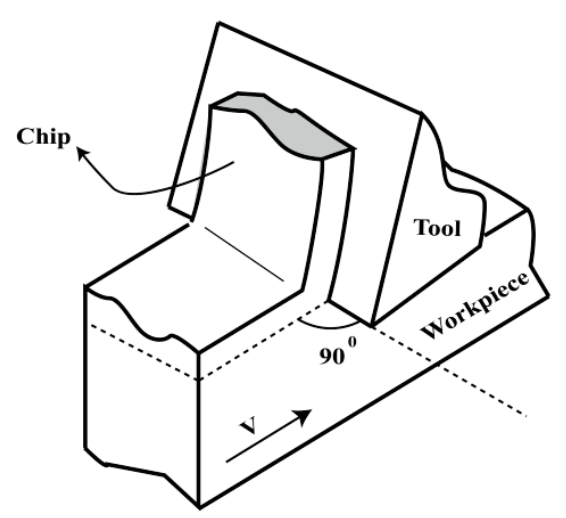

(a)

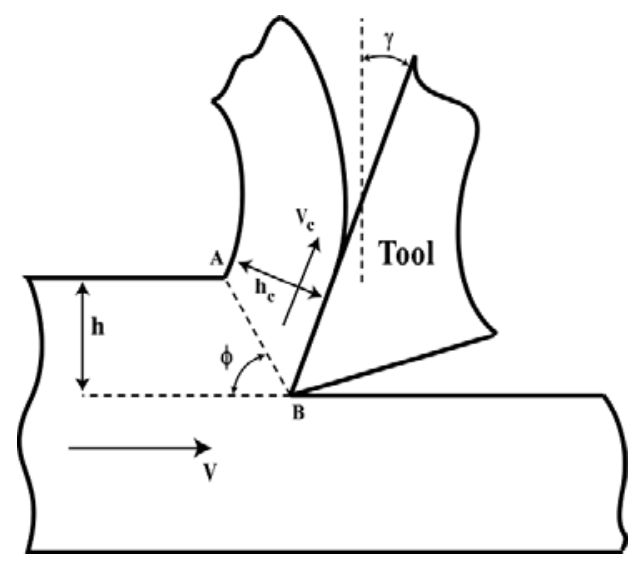

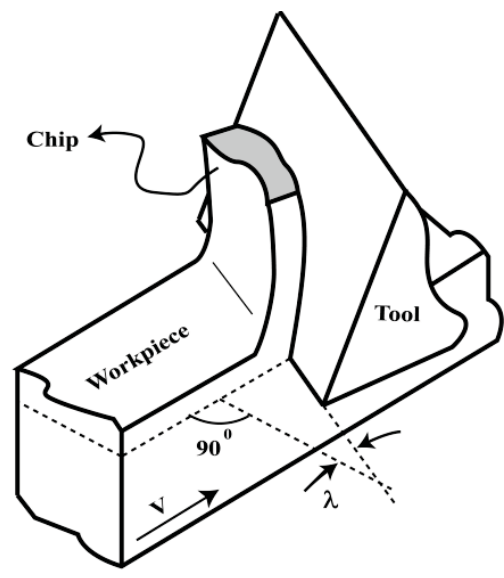

(b)

$$
\begin{aligned}
& \phi \quad \text { - Shear Plane Angle } \\
& \text { h - Uncut Chip Thickness } \\
& \mathbf{h}_{\mathbf{c}} \text { - Cut Chip Thickness } \\
& \mathbf{V}_{\mathbf{c}} \text { - Chip Velocity } \\
& \text { V - Cutting Speed } \\
& \gamma \text { - Tool Rake Angle }
\end{aligned}
$$

(c)

Figure 2.1: (a) Orthogonal cutting model (b) Oblique cutting model (c) General 2D representation of the cutting process with the variables of the cutting process.

(Reproduced from Boothroyd and Knight, 2006)

cutting process as shown in Figure 2.2. In the force circle method (commonly called Merchant's force circle diagram), the cutting process is analyzed using a free body diagram of individual forces during the cutting process. A single resultant force vector $R$ acting on the tool tip represents the net effect of all the forces acting on the tool. An explicit assumption is that the shear plane angle takes on a value that minimizes the work 


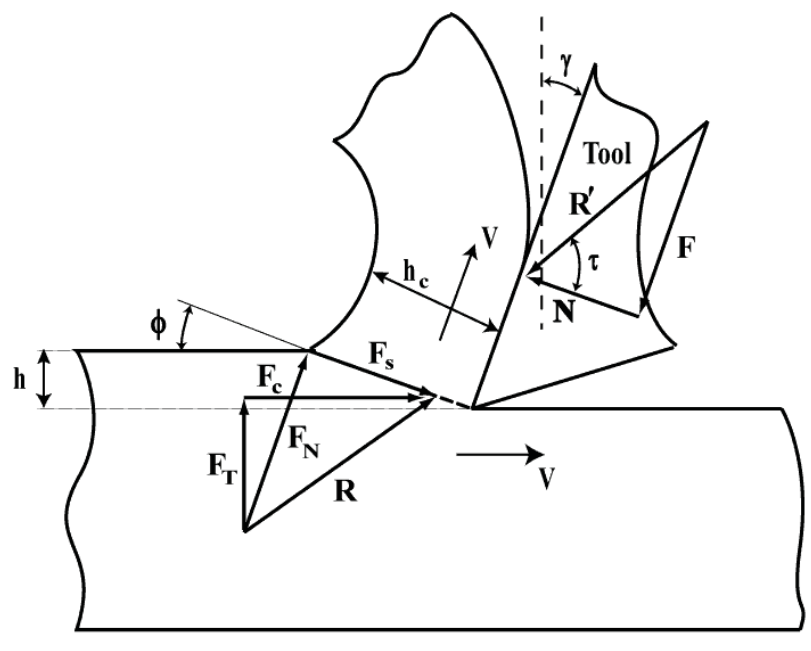

(a)

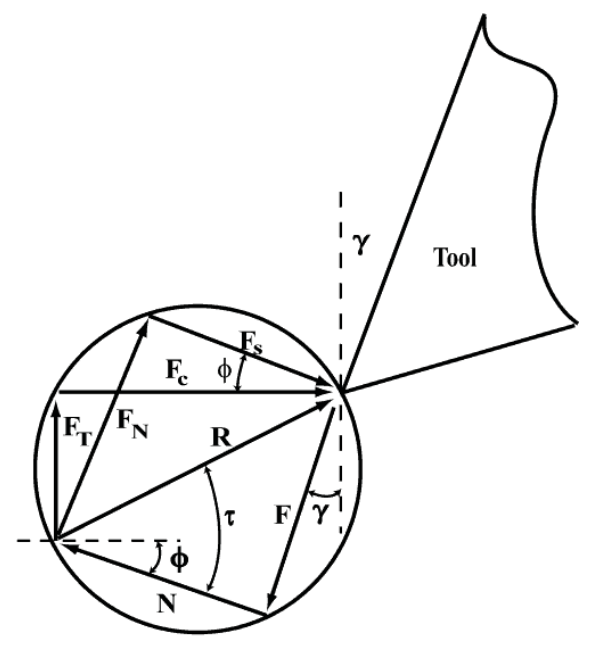

(b)

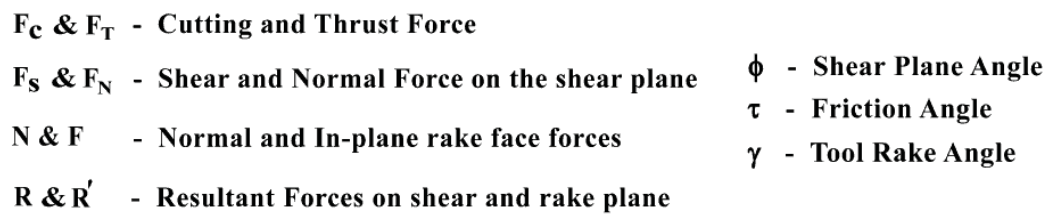

Figure 2.2: (a) Merchant's force considerations in machining (b) Merchant's force circle diagram showing the resultant force at the tool tip.

done in cutting a specific work material. This value of the shear plane angle is dependent on the tool geometry and cutting conditions used. Merchant's model for the shear plane angle is given as

$$
\emptyset=45^{0}+\frac{\gamma}{2}-\frac{\beta}{2}
$$

where $\phi$ is the shear plane angle, $\gamma$ the tool rake angle and $\beta$ the friction angle.

Another assumption of Merchant's force model is that all of the shearing action is confined to a single plane of deformation. Lee and Shaffer (1951) used a different approach and modeled the cutting process based on the plasticity theory. The Lee and 
Shaffer model assumes that plastic deformation extends over a defined shear zone represented by $\triangle \mathrm{ABC}$ as shown in Figure 2.3. A homogeneous state of stress is assumed within the shear zone with no work hardening effects. All shearing action is believed to occur along parallel lines of slip within the shear zone. Lee and Shaffer's model also assumes that the maximum shear stress is oriented in the same direction as the shear plane AC. Using the Mohr's stress circle to determine the state of stress in the shear zone, Lee and Shaffer's model is expressed as

$$
\varnothing=45^{0}+\gamma-\beta
$$

One limitation in the assumptions of Lee and Shaffer's model is the idealization of the stress state in the shear zone. By assuming a uniform stress state, the model ignores work hardening affects and explicitly considers a case of zero shear outside the bounda-

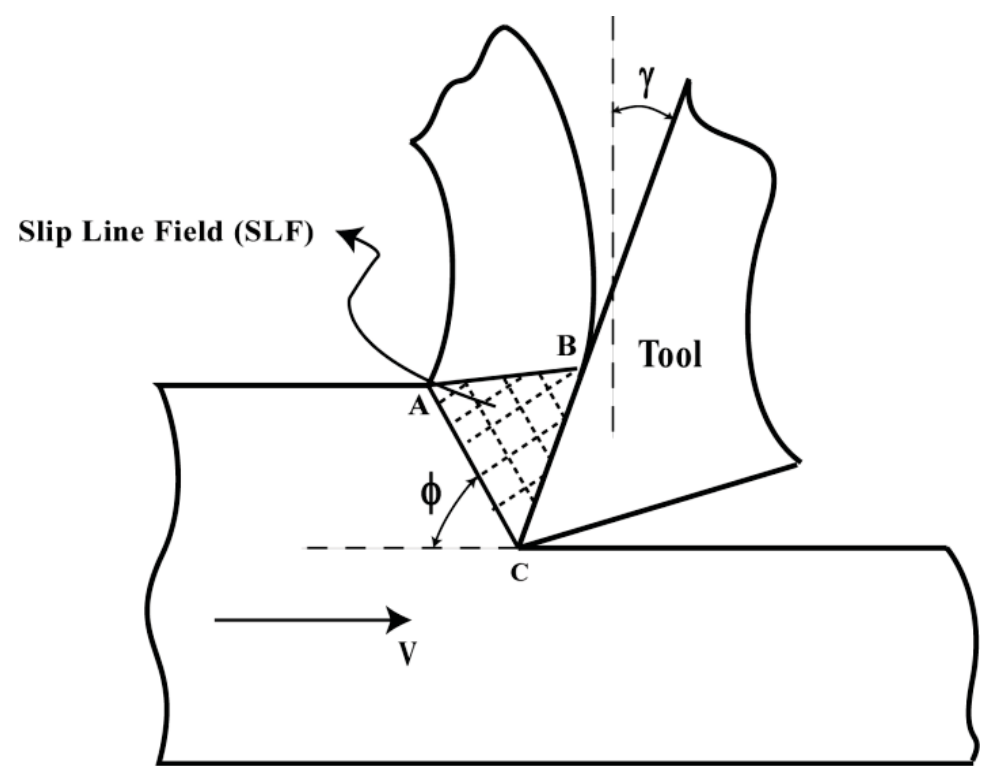

Figure 2.3: Lee and Shaffer's slip line field model. 
ries $\mathrm{AB}$ and $\mathrm{AC}$ of the shear zone. Experimental photographic evidence however (Cook et. al., 1951) contradicts this theory. High-speed photographs of the shear zone during cutting indicate that the shear zone is more widely distributed and assumes a curved shape at its boundaries. The extent and curvature of the shear zone depends on the cutting conditions, i.e., rake angle, depth of cut, cutting feed, etc.

Zorev (1966) pointed out inconsistencies of both the single shear plane and uniform stress slip-line theoretical models. He concluded that both models are limited in their assumptions; the slip-line model does not consider the practical aspect of work hardening (and thus no stress gradient) during machining while the single shear plane model ignores the enormous deceleration needed for the work material to change velocities instantly from $V$ to $V_{c}$. Zorev proposed a shear plane model as shown in Figure 2.4 (a) which considers the shear zone as being encompassed by a series of distinct shear planes each defined by progressively varying shear stress values and velocity gradients. A simplified version of this model is as shown in Figure 2.4 (b) and represents the shear zone as a series of straight shear planes each with a different shear angle.

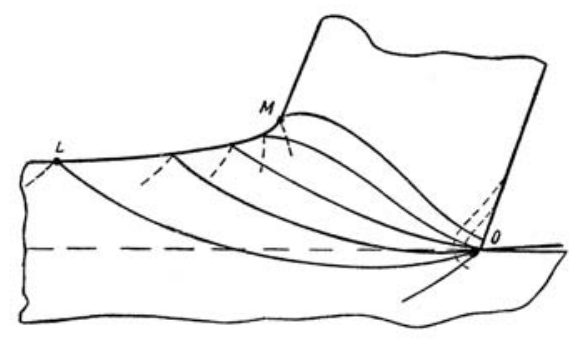

(a)

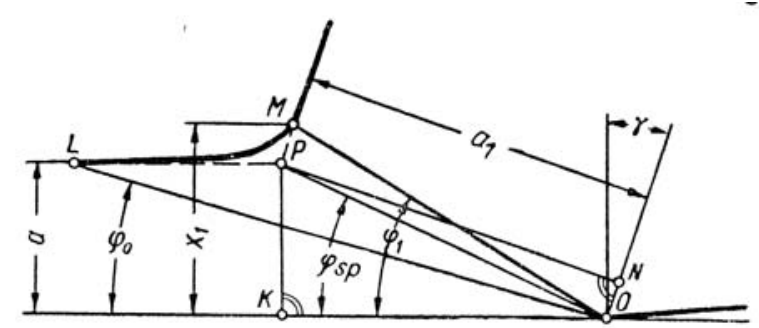

(b)

Figure 2.4: (a) Zorev's curved slip line model (b) The simplified version of the curved slip line model. (Zorev, 1966) 
Palmer and Oxley (1959) experimentally studied the cutting process using a cine film technique. Their results corroborate the multi-shear plane theory. They also conclude that tool chip contact occurs some distance away from the tool tip resulting in a kinematically dead zone at the tool tip. It was incorrectly assumed that no further plastic deformation occurs as the chip contacts the tool face and the secondary deformation observed is largely due to elastic chip flattening at this point. However, as proven conclusively from the later experimental work of Wallace and Boothroyd (1964), the chip continues to undergo plastic deformation via shear upon contact with the tool rake face. A similar theory was included in Zorev's (1966) work on predicting the shear zone area.

Thus, two major deformation zones called the Primary Deformation Zone (PDZ) and a Secondary Deformation Zone (SDZ) can be identified in metal cutting. The latter occurs because of shear due to the contact friction conditions between the chip and the tool rake face. Based on experimental observations, Roth and Oxley (1972) developed a slipline field model using velocity dependent material flow lines identifying definitive PDZ and SDZ areas. Based on the latter set of slip-line field models, the orthogonal cutting process can then be represented as shown in Figure 2.5.

Depending on the subject matter under consideration, both the single shear plane models as well as slip-line models have been used alternatively by researchers to study the cutting process. Aside from the seminal work already mentioned, several excellent analytical models characterizing cutting mechanics are available. A listing of analytical models is presented elsewhere by Shaw (2005) and will not be discussed here for brevity. 


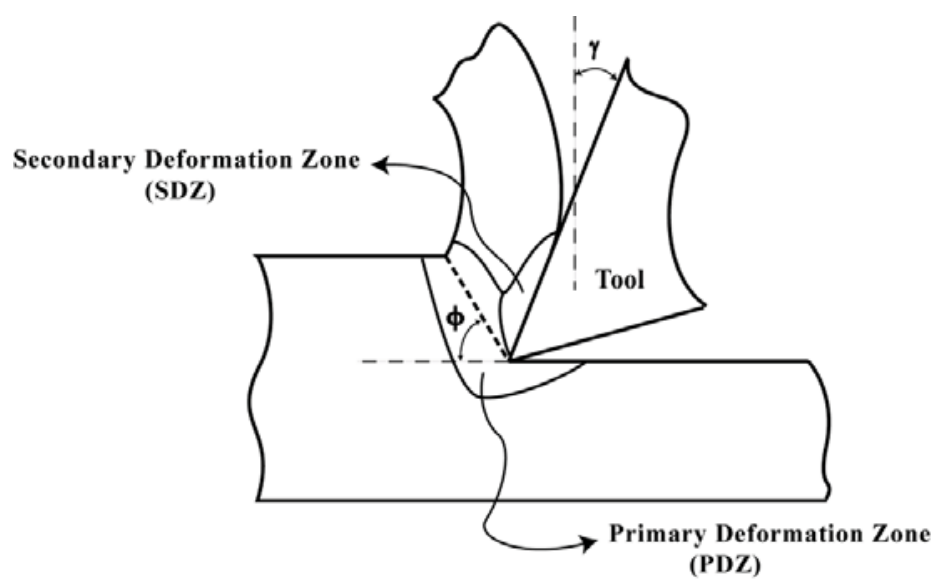

Figure 2.5: Orthogonal schema showing primary and secondary deformation zones.

\subsection{Chip Formation and Classification}

Considerable debate exists in the literature on the exact nature of the chip formation process. Subjectively, chip formation occurs either by fracture ahead of the tool or due to plastic flow with no material separation.

Chip formation due to fracture of the work material ahead of the tool - most easily visualized as splitting of wood by a hatchet - has been postulated in early works on machining theory (Finnie, 1956). While the fracture theory can be very easily applied to chip formation during machining of brittle materials, it has generated a significant argument against it when applied to the machining of ductile materials (Ernst, 1938) due to the non-existence of any visible fracture or cracks during machining. Cook et al. (1954) postulate that since there is generation of a new surface with material removal, a minute amount of fracture at the tool cutting edge is inevitable even during cutting of ductile metals, although no evidence of fracture exists. Their hypothesis considers the work ma- 
terial and chip as analogous to a series of plates each organized by grain depths (layers numbered 1-10) as shown in Figure 2.6. Due to the presence of a finite edge radius even with the sharpest of tools, each plate near the tool tip shears at a different angle but the shear angle remains constant farther away from the tip. Moreover, the stress state near the tool tip is tensile but changes to compressive at the work surface. Such a variation in shear angles and stresses should then naturally produce conditions that generate a crack ahead of the tool.

Later work from Connoly and Rubenstien (1968) also supports the theory of microcracking to some degree. One of the assumptions in their model is that the direction of material flow and separation (i.e., the chip) depends on a critical point in the flow region as shown in Figure 2.7. All material above the critical point tends to flow over the rake face as a chip with the remaining material flowing elastically under the tool edge radius. Such a theory has also been used to explain the ploughing action (Albrecht, 1960) while cutting at low uncut chip thicknesses. A more recent use of the fracture theory was used in developing a FEA formulation to model chip flow (Madhavan et al., 2000). The chip

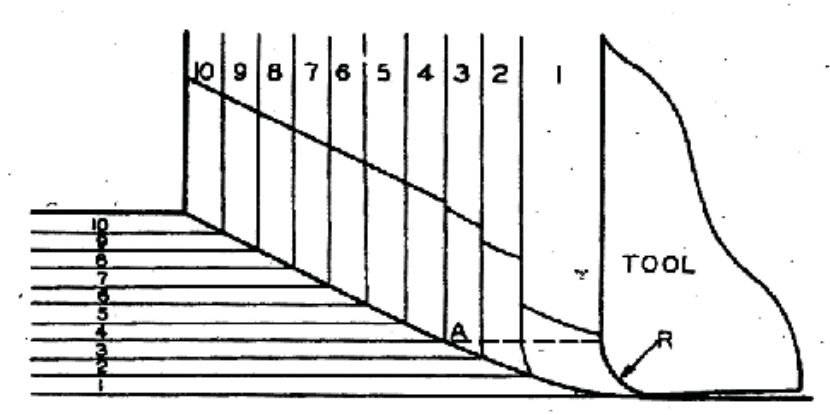

Figure 2.6: Cook's plate analogy for chip formation. (Cook et al., 1954) 


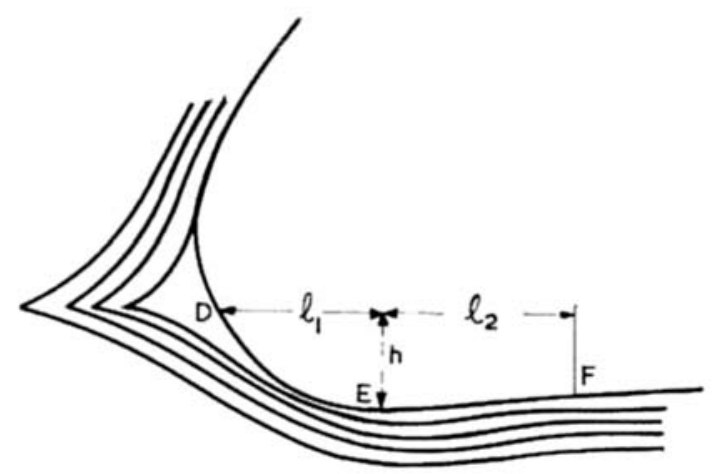

Figure 2.7: Material flow around the tool tip. (Connoly and Rubenstien, 1968)

formation is treated as a special case of wedge indentation with fracture occurring ahead of the tool. However, in the absence of a clear cleavage that emanates to the surface the fracture mode of material removal for ductile material machining incites further debate. Cook et al. (1954) speculate that the larger compressive stresses at the work surface attenuates (or re-welds) the micro-crack formed at the tool tip. While this theory has been used to characterize material removal at micro scales (Subbiah and Melkote, 2007), no information compelling the use of fracture theory vis-à-vis ductile chip formation at macro scale machining is forthcoming in the literature.

More prevalent is the second theory of chip formation: that the chip is formed as a result of plastic flow and subsequent shear with no material separation. Using this theory, Merchant (1945) and Piispanen (1938) separately developed coincidentally identical models to explain chip formation. The chip is considered as a continuous medium connected to the workpiece at the root. Both Merchant's and Piispanen's models are primarily used to explain the Type II variety of chip (discussed later in this section). 
Amongst the two models, Piispanen's rather simplistic "card deck model" analogy provides an easy illustrative approach to surmise the chip formation process. The work material can be a visualized as being deformed in parallel "lamellas" when the tool edge moves relative to the free work surface as shown in Figure 2.8. When an imaginary rectangular area $A$ in the work material moves against the tool rake face, plastic deformation induces it into parallelogram $B$. As the deformation increases, material that is cut shears from the bulk work material and continues its motion along the tool rake being aided by subsequent material being sheared immediately behind it. Schematically, a long continuous chip can then be imagined as being made up of a stack of card-like elements each similar to the parallelogram analogy and bound to one another by shear welding. The shape of the parallelogram $B$ is conjured for simplified explanation whereas the final shape is a combination of tool geometry, shear stresses and most importantly the shear

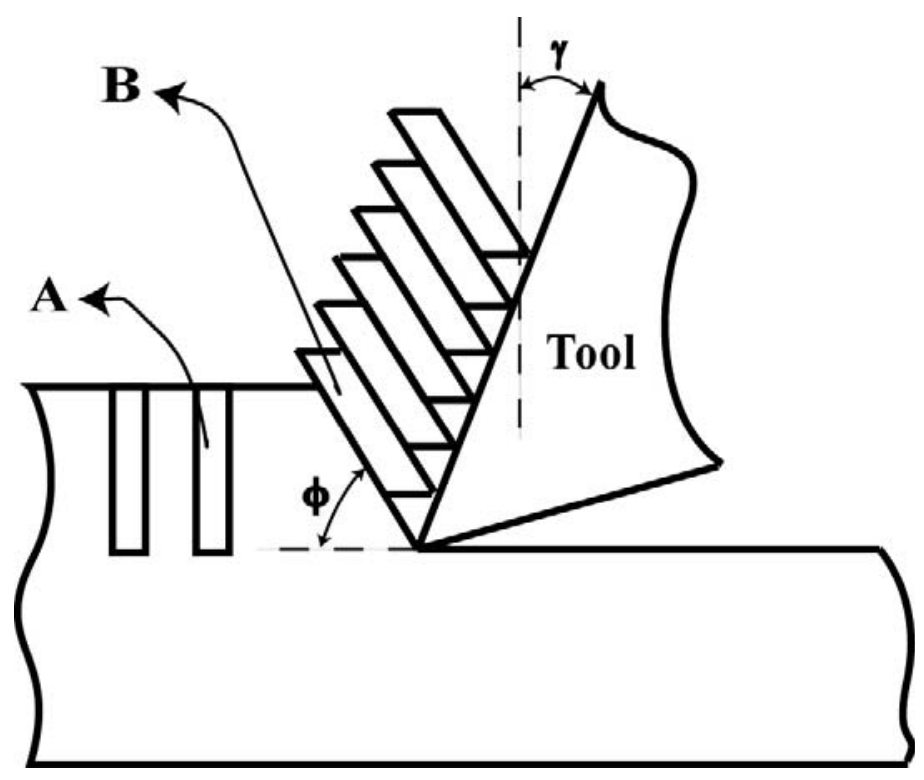

Figure 2.8: Piispanen's card deck model of chip formation. 
plane angle, $\phi$. In light of experimental photographs presented later by Cook and Shaw (1951), Piispanen's schema appears to be reasonable and a good representation of the chip formation process.

Both the Merchant and Piispanen chip theories adopt the single shear plane analytical model. Experimental evidence (Palmer and Yeo, 1963) however suggests that the chip formation process in fact does not follow the ideal single shear plane theory but rather that the shear zone is spread out. At suitable cutting conditions, however, the shear zone width contracts to a narrow band and starts to appear as a single plane (Cook et al., 1954). Thus, despite its arguable limitations (Astakhov, 2005), the single shear plane model serves as a good representation due to its simplicity and can be used as a competent first approximation of the chip formation process.

In practical machining, depending on the cutting conditions the chip may assume one of several forms. The general categorical forms (Ernst, 1938) widely in use today are:

1) Type 1 or Discontinuous chips

2) Type II or Continuous chips

3) Type III or Continuous chips with built up edge (BUE)

4) Type IV Segmented Chips

The last form is a relatively recent classification and these are the predominant chip form when machining materials with low thermal conductivity such as Titanium (Komanduri and Brown, 1981). Figure 2.9 illustrates the afore mentioned chip types. 


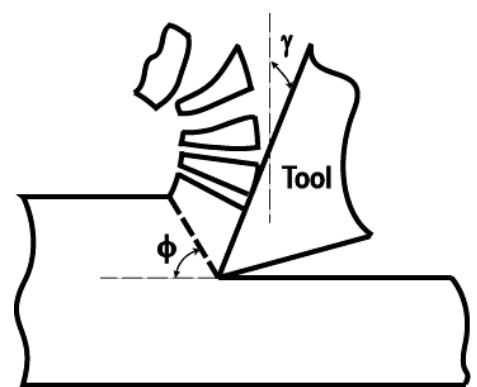

(a)

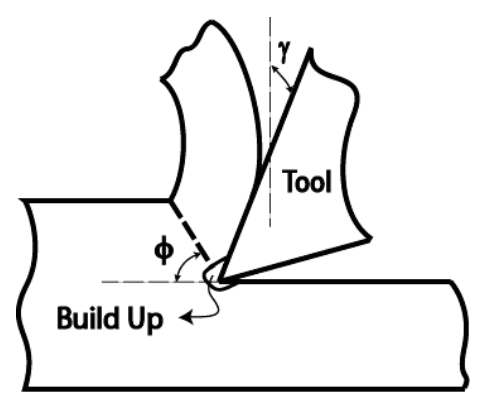

(c)

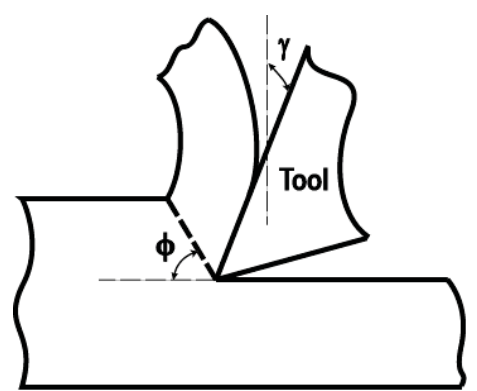

(b)

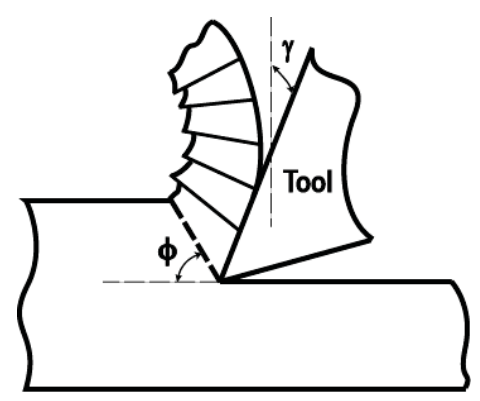

(d)

Figure 2.9: Four major chip morphologies (a) Type I (b) Type II (c) Type III with BUE (d) Type IV.

In the context of chip control research, Type 1 or discontinuous chips - those produced during machining of brittle materials - are relatively inconsequential. The nature of the process inherently serves to produce chips that are small and easily managed. Type II and Type III chips are the real area of interest for chip control research due to the difficulty in breaking such chips. Most work in the area of chip control idealize the chip flow pattern and either exclude Type III/IV chips or treat them in the same class as Type II chips. 


\subsection{Chip Kinematics}

In the last few decades, significant research has been devoted to the problem of chip control in machining. Detailed reviews (Kluft et al., 1979; Jawahir and van Luttervelt, 1993) are available on the present state of art in chip control research. In fact, a comprehensive database of relevant research in this area has been cataloged and reposited at the University of Kentucky (Jawahir, 1993).

Two basic characteristics define the chip kinematics during the cutting process: (1) The initial flow or path assumed by the chip upon formation and, (2) The curl exhibited by the chip post formation. Both processes need understanding in conjunction to be able to effectively control the chips (Kluft et al., 1979).

\subsubsection{Chip Flow}

Initial flow of the chip at the cutting edge is an important consideration since it drives the eventual form assumed by the chip. The geometry - shape and orientation - of the tool edge instigates the initial chip flow. As such, it is important to analyze the impact of the tool edge orientation towards the chip formation and flow. A detailed discussion on tool geometry is included in APPENDIX A and will not be expounded here.

Based on tool geometry considerations, two different modes of flow are possible: (1) Side flow $\eta_{\mathrm{c}}$ and, (2) Back flow $\eta_{\mathrm{b}}$. Both modes are discussed in subsequent sections. All of the discussions are based on a single point cutting tool process since multi-point tools such as those used in milling and drilling processes are generally considered extensions of the basic single toothed processes. 


\subsubsection{Side Flow}

The chip side flow angle, $\eta_{\mathrm{c}}$, is defined as the angle made by the initial chip flow direction with the primary axis of rotation (work axis in Turning and tool axis in Milling). It is measured in the plan view of the tool rake face as shown in Figure 2.10.

From a mechanics perspective, initial chip flow is assumed to be in the same direction as the resultant cutting force as given by Merchant's 2D force diagram. Predictive models of chip flow generally utilize some form of mechanistic force modeling approach to determine the resultant force vector orientation. When applied to the practical 3D machining scenario, a transformation from tool local coordinates to a global coordinate system is required to arrive at the resultant force orientation (Endres, 2005). Thus, accurate prediction of the initial chip flow is possible with accurate predictions of the orientation of the resultant force vector at the tool edge (Shaw et al., 1952).

In a flat faced free orthogonal cutting configuration (See APPENDIX A), the chip flow is normal to the tool cutting edge and the chip side flow angle $\eta_{\mathrm{c}}$ can be easily measured by drawing a perpendicular to the tool cutting edge. But, the complexity in-

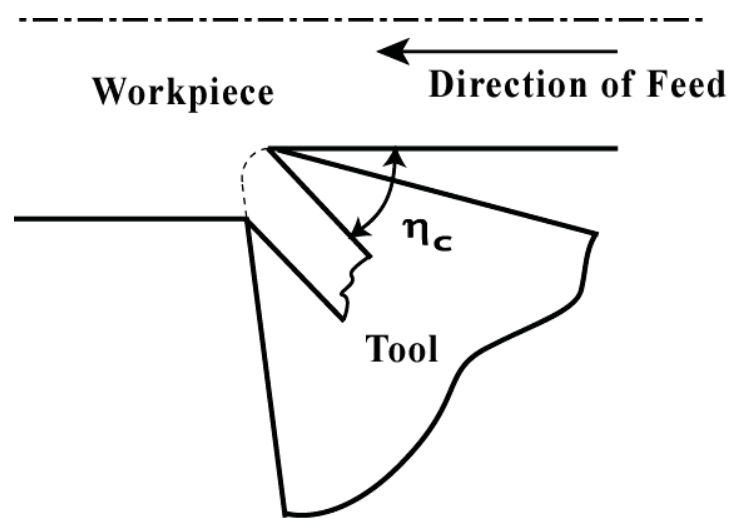

Figure 2.10: Schematic illustration of the chip side flow angle, $\eta_{c}$. 
creases in the case of a non-free orthogonal configuration wherein the tool corner radius alters the resultant force orientation and consequently the chip flow direction. It then becomes necessary also to consider the influence of the corner radius on the resultant force vector orientation to be able to predict the chip side flow.

One of the earliest models to predict the chip side-flow angle in non-free orthogonal machining was by Colwell (1954) who showed that the chip side flow direction is approximately normal to a hypothetical "equivalent" cutting edge defined by the "equivalent" lead angle, $\psi_{r}$. The equivalent lead angle can be easily derived from pure geometric considerations of the cutting process. Colwell's method defines the equivalent lead angle as the chord representing the major axis of the projected area of cut. When cutting with a corner radius the projected area is the area enclosed between subsequent feed grooves and the tool edge participating in the cutting process. The equivalent lead angle and chip side flow direction are shown in Figure 2.11.

Okushima and Minato (1959) later developed another method whereby they divided the cutting edge into finite segments and considered the chip flow from each segment as being normal to the cutting edge of the individual segment. Vectoral integration of flow due to individual segment orientation then gives the eventual averaged chip side-flow angle. The method of predicting the equivalent lead edge by dividing the edge into finite segments was also utilized in some other later work using the mechanistic approach to model the direction of the resultant cutting force. Fu et al. (1982) assumes a constant force distribution on the tool cutting edge and integrates the force values over the cutting edge to obtain the resultant cutting forces. Subramani et al. (1987) considers the edge force distribution as being proportional to the varying uncut chip thickness along the cut- 


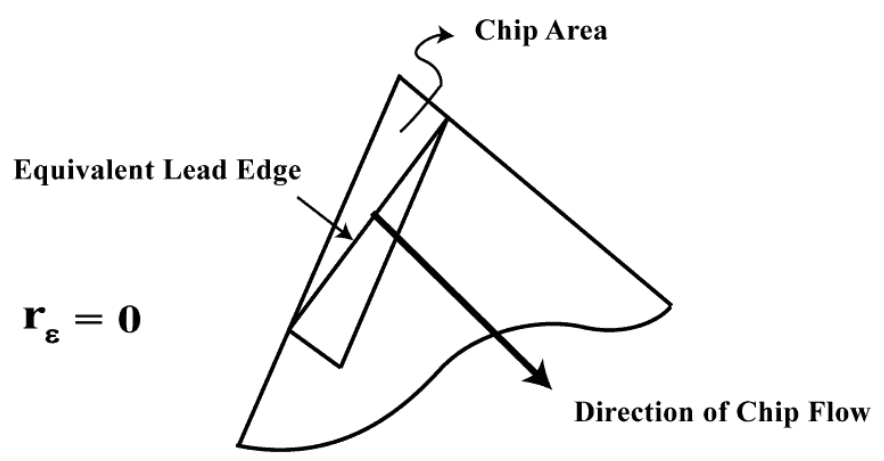

(a)

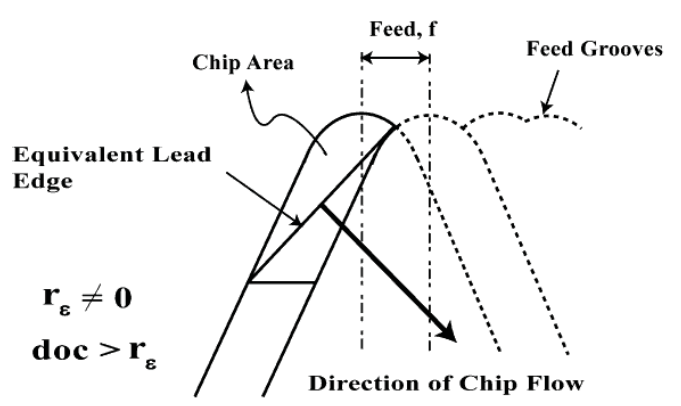

(b)

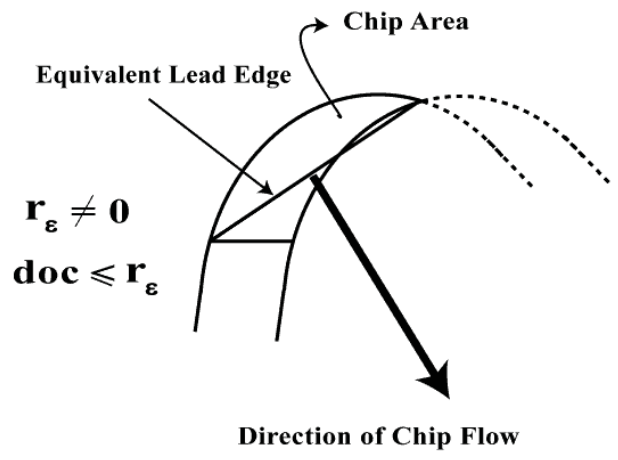

(c)

Figure 2.11: Colwell's chip flow model for (a) Sharp tool, $\mathbf{r}_{\varepsilon}=0$ (b) Corner radiused tool with doc $>\mathbf{r}_{\varepsilon}, \mathbf{r}_{\varepsilon} \neq \mathbf{0}$ (c) Corner radiused tool with doc $\leq \mathbf{r}_{\varepsilon}, \mathbf{r}_{\varepsilon} \neq \mathbf{0}$.

ting edge. The resultant force direction and consequently, the equivalent lead angle is tained by numerical integration of the forces as a function of the varying uncut chip thickness. Endres et al. (1993) use a cutting width weighted average technique along the discretized cutting edge to predict the equivalent lead angle. Any one of the methods can be used to predict the equivalent lead angle in non-free orthogonal cutting. In most cases, Colwell's geometry based method will suffice due to its easy implementation.

When extended to the oblique machining condition, the tool orientation in oblique cutting adds another degree of complexity that needs consideration for prediction of the 
chip side flow angle. A simple and very commonly cited model is the one proposed by Stabler (1951), who approximated the chip side-flow angle to the inclination angle. No account for either the corner radius or, for that matter, the cutting parameters, was included in this model. Simply put as an equation, Stabler's model can be written as

$$
\eta_{c}=\lambda
$$

where $\eta_{c}$ is the chip side flow angle and $\lambda$ is the tool inclination angle.

Stabler's model shows significant deviations at large inclinations, a fact verified by Shaw et al. (1952). Stabler (1964) subsequently introduced a corrected model with a constant of proportionality $K$ that depends on the work material properties with values between 0.9-1. Shaw et al. (1952) purport that the important tool angle for chip-side flow is the normal rake angle (also called effective rake angle in said work) rather than the inclination angle as proposed by Stabler. Of debate was the fact that since the friction force component mainly affects the chip side flow and with the frictional force being measured in the normal rake plane, the normal rake angle should then be the influencing angle for chip side flow. In the absence of a concrete theory to prove otherwise, Shaw and coworkers conceded that Stabler's rule was a very good approximation of the chip side flow especially at the lower inclination angle values normally used in machining. The matters of friction and the inadequacy of the inclination angle in Stabler's rule was revisited by Brown and Armarego (1964) who concluded that the inclination angle itself is independent of the normal shear plane and agree with Shaw's view on the consideration of the normal rake angle for predicting the chip side flow angle. Zorev (1966) however maintains that Stabler's rule holds in the case of oblique cutting. Based on experimental 
results he contends that the friction force and chip flow direction coincide well enough (within an accuracy of $2^{0}$ in his argument; Zorev, 1966; cited on page 405). It was presumed that the deviations between the experimental side flow angles and the analytical model that Shaw reported in his earlier experiments was due to built up edge or large secondary shear conditions. But it is also arguable that under practical cutting conditions, the presence of build up as well as secondary shear is bona fide and hence it can be contended that the presence of build up and friction do create deviatoric conditions for chip flow.

Since the oblique cutting model is in essence a transformation of the orthogonal cutting case, Luk (1972) used orthogonal cutting data and developed an analytical model to arrive at the chip side-flow angle. Variables included in this model are the normal rake angle, inclination angle and constants representing the tool-workpiece materials. Lin and Oxley (1972) again recast Stabler's rule in their work on development of a chip flow model and concluded that Stabler's law is a good approximation of chip side-flow angle at low inclination angles. They also contend that the deviations observed at higher inclinations are due to the measurement practice of using images projected on to the plan view of the tool rather than being measured directly in the plane of chip flow. Incidentally, Lin and Oxley's experimental results are based upon the measurement of chip flow angles from wear marks on the tool rake face and seem to better match analytically predicted results.

One unique observation from early works on oblique machining is the general exclusion of corner radius effects in the models. While researchers such as Zorev (1966) and Luk (1972) acknowledge the importance of corner radius, their work does not include the 
effects of a curvature at the tool corner during cutting. In reality, the corner radius is an obvious part of the cutting process and so must be included in the treatment of machining models. Okushima and Minato's (1959) work does consider the corner radius but overestimates the chip side flow angle largely due to the assumption that the varying chip area across the cutting edge length contributes equally to the chip side flow. Using empirical methods Spaans (1970) distinguished the chip side flow into two separate but superposed events - the effect of the corner radius and that due to the inclination of the straight cutting edge. Spaans developed an equation for the side flow of a chip which is given as

$$
\eta_{\mathrm{c}}=\frac{33.3 \mathrm{r}_{\varepsilon}}{\mathrm{d}}+3.3+\kappa \lambda
$$

where $r_{\varepsilon}$ is the tool corner radius, $d$ is the depth of cut, $\kappa$ is a material constant $(\sim 0.5-1$ for steels) and $\lambda$ is the tool edge inclination angle.

When the tool corner region is involved in the cutting action, a part of the secondary cutting edge is also included in the process (Nedess and Hintze, 1989). Hu et al. (1986) combined the effects of the main cutting edge with that of the end cutting edge. Similar to Colwell's model, their model proposes using an equivalent cutting edge in an oblique cutting configuration. Compared to earlier results from Stabler's method applied to large inclinations, their results indicate that a much closer match can be obtained between predicted and experimental chip flow angles by using the inclined equivalent cutting edge model. Young et al. (1987) developed a predictive force model to include the corner radius effects. In an approach similar to Usui and Minato's earlier work for non-free orthogonal cutting, the cutting edge is divided into small linear segments and analyzed. 
Chip flow at each segment is approximated to the segmental inclination according to Stabler's rule. Although the inclination angle is discussed briefly, Young's experimental design considers only the case of orthogonal cutting. So the inclination angle is excluded in the final model for chip flow. In doing so, the model becomes an extension of Okushima and Minato's earlier work with the difference being that Young's work includes the effects of the varying chip cross sectional area across the corner radius. Wang and Mathew (1995) and later Arsecularatne et al. (1995) extended this work by including the effect of varying inclination angle.

\subsubsection{Back Flow}

The chip back flow angle (also called chip streaming), $\eta_{\mathrm{b}}$, is defined as the angle formed by the chip with the rake face plane and measured in the plane parallel to the tool axis as shown in Figure 2.12.

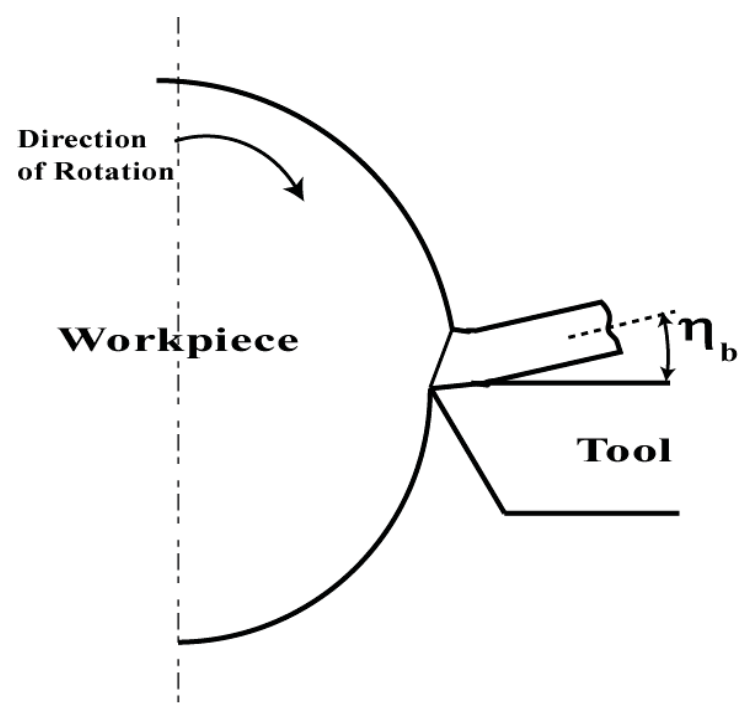

Figure 2.12: Schematic illustration of chip back flow angle, $\eta_{b}$ (chip streaming). 
Loss of contact between the chip and the tool rake face is the primary reason for the chip back flow. From tribological considerations of the cutting process, the loss of contact is attributed to both the normal and shear stresses, which vary over the chip contact length ranging from maximum at the tool tip to zero at the end of the natural chip contact length (Usui and Takeyama., 1960). When the natural chip contact length reduces or is restricted, the immediate result is to cause the chip to stream or lose contact with the rake face.

Using slip-line field analysis, Jawahir and Oxley (1988) have developed what appears to be the only quantitative relationship for the chip back flow available in the literature and it is expressed as

$$
\eta_{\mathrm{b}}=\tan ^{-1}\left[\left\{\frac{\cos \alpha}{\cos \gamma \sin (\alpha+\xi+\gamma))}\right\}-\cot (\alpha+\xi+\gamma)\right]
$$

where $\alpha$ is shear stress state given by $2 \alpha=\cos ^{-1}(\tau / K)$, with $\tau$ being the shear stress and $\mathrm{K}$ the flow stress of the material, $\gamma$ is tool rake angle and $\xi$ is the center fan angle of the slip line filed and is expressed as

$$
\xi=\cos ^{-1}\left[\frac{\left(\cos \gamma-\left\{t_{1} / w\right\}\right)}{\sqrt{2} \cos \alpha}\right]-\left[\frac{\pi}{2}+\alpha+\gamma\right]
$$

with $t_{l}$ being the uncut chip thickness and $w$ the length of restricted contact length. 
Jawahir and Oxley (1988) also presented experimental evidence of the relationship between the uncut chip thickness and the restricted contact length. They found a marked increase in the back flow angle at combinations of high uncut chip thickness and low contact lengths. The real challenge in predicting the chip back-flow angle is the difficulty in accurate measurement of the chip contact length as acknowledged by Chao and Trigger (1959) in their seminal work on thermal effects in machining. Friction conditions on the rake face vary infinitely depending on tool-work material combinations, build up, lubrication and cutting condition (Ernst and Merchant, 1941). Thus, even seemingly minor variants such as tool land (Sikdar et al., 1991) and edge chamfer could potentially result in chip back flow. In general, straight chip back flow is very rare since loss of contact with the tool rake face usually results in a pronounced curl (Henriksen, 1954) rather than a straight chip. This may partly help explain the relative lack of research interest in straight chip back flow.

\subsubsection{Chip Curl}

Once past the tool edge, the chip exhibits a curl due to the influence of either the corner radius, angle of inclination or a combination of both. Straight chip flow is rare when cutting with a corner radius. Moreover, straight chips are relatively easy to control. Chip curl on the other hand poses a greater challenge to predict or control since the final form (curl) can change even with minor changes in the cutting condition. As such, a major part of chip control research has concentrated on understanding the curling mechanism rather than the flow. 
Hahn (1953) states that the chip is "born" curled in the shear region with the curl being unaffected by the tool-chip contact. Palmer and Oxley's (1959) work seems to augment this theory. Using results from a cine film experiment, they trace the path of a single grain of material from the shear zone to the chip end. Because a single unique arc connected both the start and end-points, it was concluded that the chip curvature originates in the shear zone. To recollect, this work assumes the single shear plane theory and that no secondary deformation occurs on the chip along its path over the tool rake face. Disagreeing with Hahn's assertion, Ponkshe (1967) opines that the chip is straight only until it leaves the tool edge. Using the slip-line field theory, he asserts that the final chip form (curling) depends on the tool-chip contact length. Thus, he concludes that the chip is not "born" curled, but assumes the curvature more due to a forced nature - because of the secondary shearing owing to the frictional force - after it has passed the tool edge. The single shear plane theory was itself disproved in other reports (Cook et al., 1951) where the highlight was the curved ends of the shear zone. Without the curvature of the shear plane, the basic assumption of the cutting model with Type 2 chip - that of material continuity - becomes invalid and the cutting model changes to that of discontinuous chip formation with fracture (van Luttervelt, 1976). If the chip curl was in fact a result of the shear zone, then the assumption of a single straight shear plane weakens the "borncurled" theory. However, lending credence to Hahn's assertion Spaans (1970) concludes that the natural chip curl is a result of a moment at the chip root. He reasoned that such a moment could be due to obstructive forces on the chip flow, including friction on the tool rake. Under such forces, the shear plane re-orients itself to restore the equilibrium of cut- 
ting forces. Thus, the debate on the exact mechanism of chip curl appears divided amongst researchers.

As with chip flow, chip curl can be classified into two distinct forms: (1) Chip sidecurl and, (2) Chip up-curl.

\subsubsection{Chip Side-Curl}

Chip side-curl or the C-shaped chip, is the curvature of the chip as measured in the plan view of the rake face plane and as shown in Figure 2.13.

In a very early work that sought to explain curvature in chips, Pekelharing (1964) terms it as the "sabre curve" form of chip. Pekelharing reasons that the chip side-curl is a manifestation of the shear plane orientation and that in oblique cutting, the shear plane takes on a helical shape. Spaans (1970) notes that sideward curvature of the chip arises due to a change in momentum across the two edges of the chip. This momentum change is due to the velocity difference that exists across the two edges of the chip. Nakayama

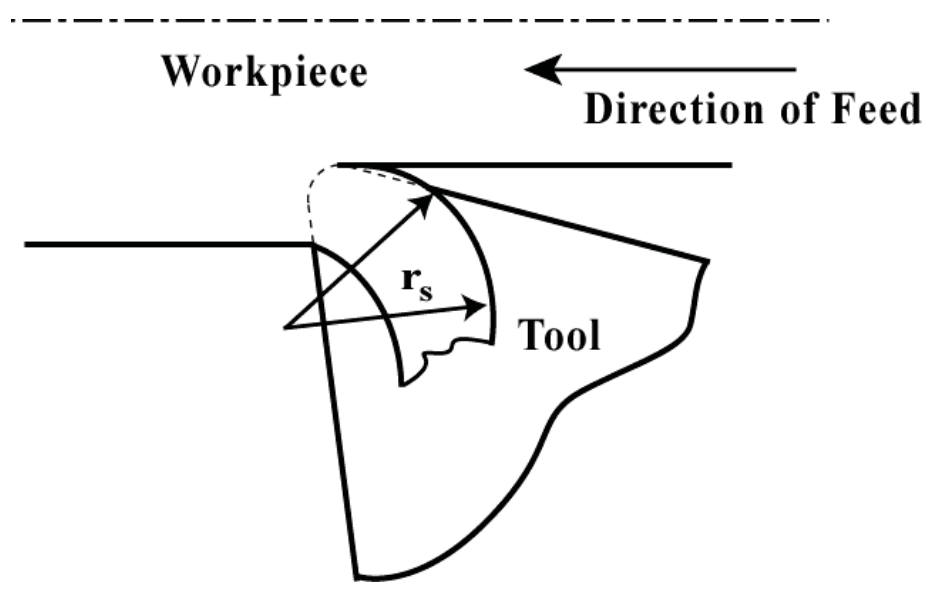

Figure 2.13: Schematic illustration of chip side-curl. 
(1972) identifies several other factors such as the stress state in the shear plane, cutting parameters and cutting-edge geometry, all of which can lead to a cutting speed gradient along the chip width. In a later work, Nakayama and Arai (1992) also include the lateral flow of the chip as well as the variation of chip cross sectional area into the list of variables affecting side-curl of the chip. van Luttervelt (1976) cites three main factors for either of the chip curl behaviors:

1) Cutting edge is not straight

2) Primary motion is not rectilinear

3) Cutting edge inclination

Most of the reported works on side-curl of the chip are experimental in nature with very few analytical models available. In the case of pure side-curling, Spaans (1970) states that the side-curl radius is approximately equal to the workpiece diameter when the corner radius is not involved in the cutting. Arai (2001) developed an empirical equation for the chip side-curl radius based purely on the chip area and it is given as

$$
\frac{1}{\mathrm{r}_{\mathrm{s}}}=\frac{0.7}{d}-\frac{0.09}{h}
$$

where $\mathrm{r}_{\mathrm{s}}$ is the side-curl radius, $d$ is the depth of cut and $h$ is the uncut chip thickness.

Bhaktavachalam and Venuvinod (1973) express the chip side-curl radius $r_{s}$ based solely on the workpiece geometry as

$$
r_{s}=\frac{R_{o}+R_{i}}{R_{o}-R_{i}}\left(\frac{d}{2}\right)
$$


where $R_{o}$ and $R_{i}$ are the outer and inner radius of the work material and pertains to the two ends of the depth of cut while $d$ is the depth of cut.

What is apparent though is that prediction of chip side-curl radius cannot be a function of geometric and process parameters alone. Clearly, the shape of the shear plane and the chip deformation (in the primary and secondary zones) also need consideration as the initial impetus to the eventual form of the chip. Under non-free orthogonal and oblique machining conditions, the shear plane assumes complex orientations based on the cutting parameters as well as geometric orientations of the tool (Usui and Hirota, 1978). It then follows (Spaans, 1970) that the shear plane orientation tends to create a moment that causes the chip to curl sideways. However, the matter of predicting shear plane orientation in oblique machining is complex (van Luttervelt, 1976) and involves understanding of the stress states, which do not have a unique solution in a dynamic process such as cutting. A change in momentum across the chip can also occur due to the variation of the friction force component across the rake face, as is the case in non-free orthogonal or oblique cutting conditions (Usui and Takeyama, 1960). A side-curl of the chip can then be expected in such a situation. DeChiffre (1992) illustrates the effect of frictional force variation on the side-curl using a simple orthogonal cutting experiment with a tool having a varying land along the cutting edge.

\subsubsection{Chip Up-Curl}

Chip up-curl is the curvature attained by the chip when viewed (and measured) in the plane parallel to the tool axis. Figure 2.14 illustrates the chip up-curl. 


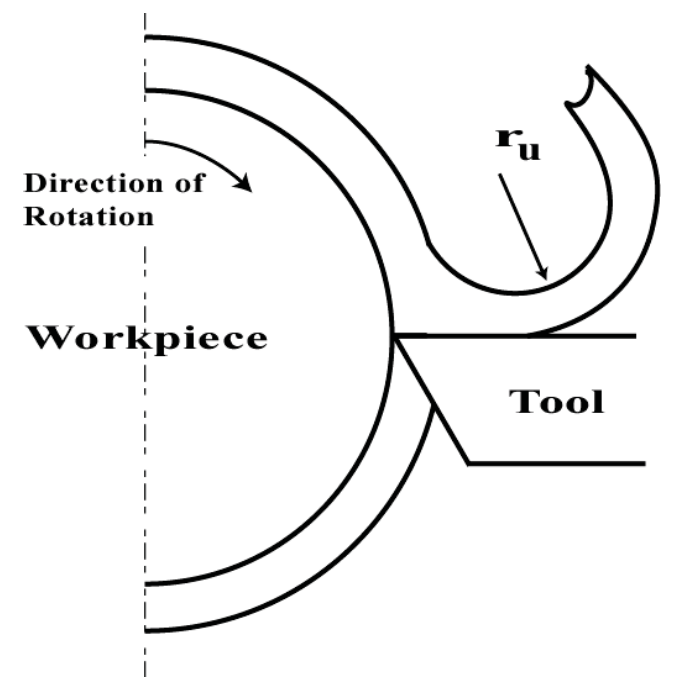

Figure 2.14: Schematic illustration of the chip up-curl.

As with the side-curl, the up-curl is also regarded as a manifestation of the shear plane orientation (Pekelharing, 1964). Albrecht (1960) considered the chip up-curl to be the result of the compressive stresses on the underside of the chip due to interactions between the tool-chip pair. He speculated that the compressive stresses are due to the ploughing effect, where the excess material near or around the cutting edge flows into the chip thereby causing an increase in compressive stresses on the chip underside. Using cine methods to examine the chip flow around the cutting edge, Palmer and Yeo (1963) claim that metal flow near the tool tip as espoused by Albrecht is not admissible and propound the possibility of a dead metal cap at the cutting edge, which can lead to an up-curl in the chip.

Another unique characteristic of the cutting process is the existence of a built up edge near the cutting edge. The material build-up seen near the cutting edge varies in form and structure with changes in machining conditions (Higginbotham and Gogia, 1961). 
Palmer and Yeo explicitly state that the dead metal cap is distinctly different from the built up edge. When machining with a dead metal cap, a built up edge or for that matter, a kinematically dead zone (Palmer and Oxley, 1959), the natural contact of the chip with the rake face occurs some distance away from the cutting edge. Given that the presence of the minute amount of material present at the cutting edge varies over the cutting process, it is but natural that the chip contact length also varies during cutting. The variation in the contact length induces an upward curvature in the chip flow (Worthington and Redford, 1973). An early example relating chip contact length to up-curl is the work of Klopstock (1925). Klopstock proposed a new tool design with the rake face ground away to reduce friction on the tool face. In doing so, the eventual chip flow showed a marked tendency to flow more gradually into the tool rake face. More significant, the results also revealed a pronounced up-curl in the chip. In hindsight, Klopstock's (1925) modified rake face design to minimize friction in effect altered the natural contact length of the chip and resulted in a chip up-curl, a fact pointed out by Chao and Trigger (1959).

To further elucidate the effect of varying the contact length on chip up-curl, Worthington (1975) used a groove running parallel to the cutting edge to mimic the restricted contact effect. A relationship was established between the uncut chip thickness and the chip up-curl radius. More recently, Jawahir (1988b) reviewed the influence of different groove geometries on up-curl. His results indicate an increased up-curl with wider grooves. Moreover, he also reports that the up-curl is increased at higher depths of cut with the side-curl being somewhat attenuated. Further, the up-curl radius reduces with increasing feed leading to chip streaming at high feeds. 
Taking the contact length and the presence of a dead metal cap into consideration as shown in Figure 2.15, van Luttervelt (1976) arrived at an expression for the up-curl radius $r_{u}$ as

$$
r_{u}=\frac{w^{2}+2 u w \sin \gamma+u^{2}}{2 u \cos \gamma}
$$

where $\mathrm{w}$ is the tool chip contact length and $\mathrm{u}$ is the height of the kinematically dead zone ahead of the tool. The tool rake angle is given by $\gamma$.

Several other aspects of machining have also been shown to affect the chip up-curl. For example, Ee et al.(2003) report that the wear marks on the tool rake face influences chip up-curl.

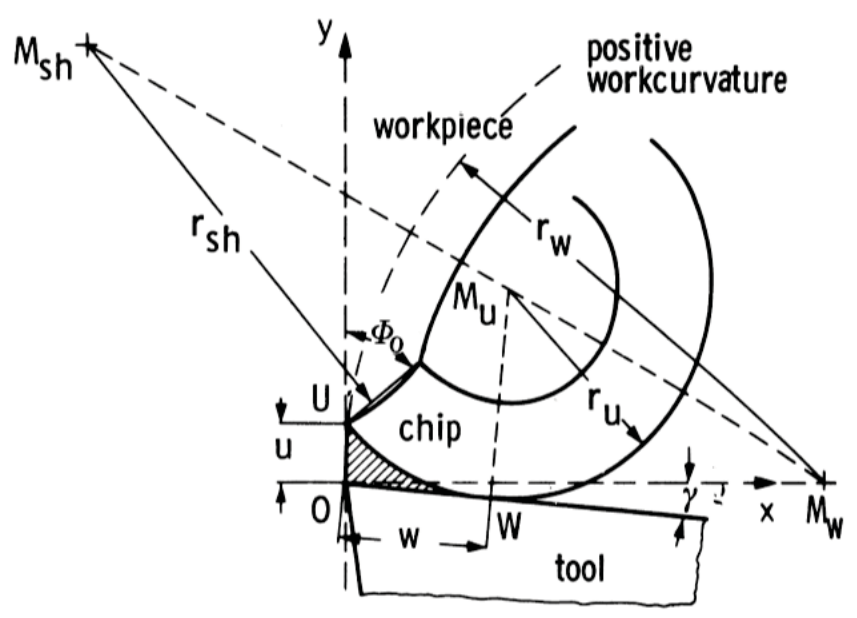

Figure 2.15: Up-curl radius as a function of the contact length. (van Luttervelt, 1976) 


\subsubsection{The 3-Dimensional Chip Flow}

In practical machining conditions, chip flow and chip curl patterns rarely occur as pure straight, side or up-curl forms. Most chips produced are 3-Dimensional in form with the final shape defined primarily by the geometry of the cutting tool. Nakayama and Ogawa (1978) identified the 3D chip form as a combination of three basic 2D chip forms - the side-curl, up-curl and the side flow. Combinations of the three basic forms results in a 3D helical loci defining the chip form and starting at the tool tip as shown in Figure 2.16 .

The spatial orientation for the helix depends on its axis. In a subsequent work, Nakayama and Arai (1992) arrived at the orientation of the helix axis based on the tool-chip contact area. The chip contact area is responsible for the velocity gradient that results in chip curl. Since the chip contact area is dynamic and complex to model, Nakayama and

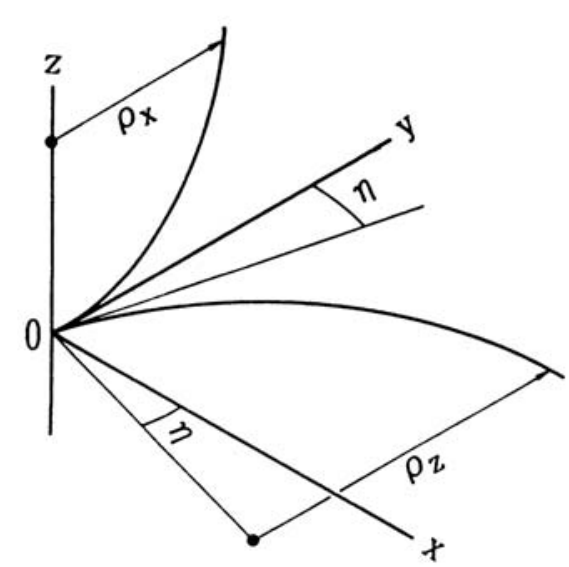

(a)

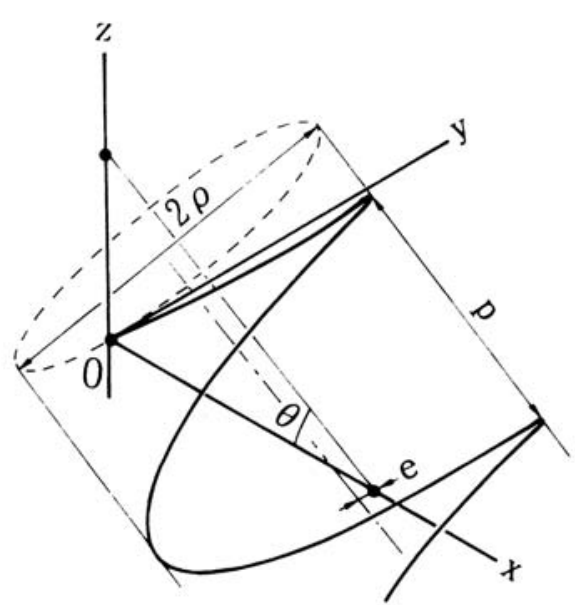

(b)

Figure 2.16: The 3D chip flow as (a) Individual side and up-curl components (b) Combined helix form based on the individual components. (Nakayama and Arai, 1992) 
Arai simply assume that a hypothetical line called the Tool-Chip Separation Line (TCSL) delineates the end of the contact zone. No exact solution is proposed for the TCSL. It is presumed that the final 3D chip form occurs only after the chip passes the TCSL. The helix axis is assumed to be oriented at an angle to the TCSL and this orientation decides the general direction of 3D chip flow.

From a survey of the literature, it appears that there is a paucity of predictive 3D chip flow models. It is speculated (Jawahir and van Luttervelt, 1993) that the main reason for the lack of predictive models on 3D chip flow is the difficulty in even arriving at a robust 2D model that accounts for the combined effects of all of the influencing variables. Compounding the topic is the complication arising from extending the classical definitions of $2 \mathrm{D}$ chip morphology to the $3 \mathrm{D}$ case. To emphasize this, Kharkevich and Venuvinod (1999) offer six different alternative hypotheses to the classical 2D definitions of side and up-curl when applied to a 3D scenario. Based on their tests of the hypotheses, direct application of 2D chip form definitions to $3 \mathrm{D}$ cases creates many ambiguities that inhibit an adequate treatment of the 3D chip form.

While most models tend to reduce the $3 \mathrm{D}$ chip flow problem to $2 \mathrm{D}$ forms, some insightful works exist on the analysis of the complete 3D chip form. Among the notable ones is Jawahir's (1991) experimental work investigating 3D chip flow. The variations in 3D chip morphology (due to changes in each of the individual constituents of the 3D flow) due to variations in cutting conditions were characterized using high speed photography techniques. Li et al. (1997) consider the unique case of side-curl dominated spiral chip for analysis. They attribute the 3D form due to restrictions in the path of a side- 
curling spiral chip. In their specific case, the restriction was due to the presence of the rotating workpiece.

Using a more complex approach, Ghosh et al. (1998) modeled the 3D chip form as a geometric spiral. The axis of the 3D chip with combined side and up-curl is modeled as a spiral geometrical form in a three dimensional space. Variations in chip morphology are treated by allowing the chip curl (side and up) radii to continuously change along the spiral. Batzer and Sutherland (2001) used a similar approach to predict the final chip form by considering a spiral locus to define the chip growth vector in orthogonal cutting. In an interesting approach to the problem, they use numerical methods to produce virtual chip forms. Their results indicate that the growth of the chip form also depends on the geometry of the obstructions to chip flow. Balaji and Jawahir (2003) studied the effect of tool-chip tribological interactions on 3D chip forms. They conclude that the transient tool-chip friction conditions coupled with other factors such as tool geometry, cutting conditions etc. lead to varying chip morphologies during machining.

\subsubsection{Breakability of Chips}

Chip breakage forms the second and often neglected aspect of chip control. Jawahir (1988a) uses the term "total chip control" as a means to define the twin processes of controlling the flow of the chip and breaking it into small manageable units. An illustration with the constituents involved in total chip control is shown in Figure 2.17.

Work material properties remain the most fundamental consideration in chip breakage. Brittle materials producing the Type 1 or discontinuous chips pose very little challenge for inducing breakage. However, in ductile materials that result in Type 2 or 


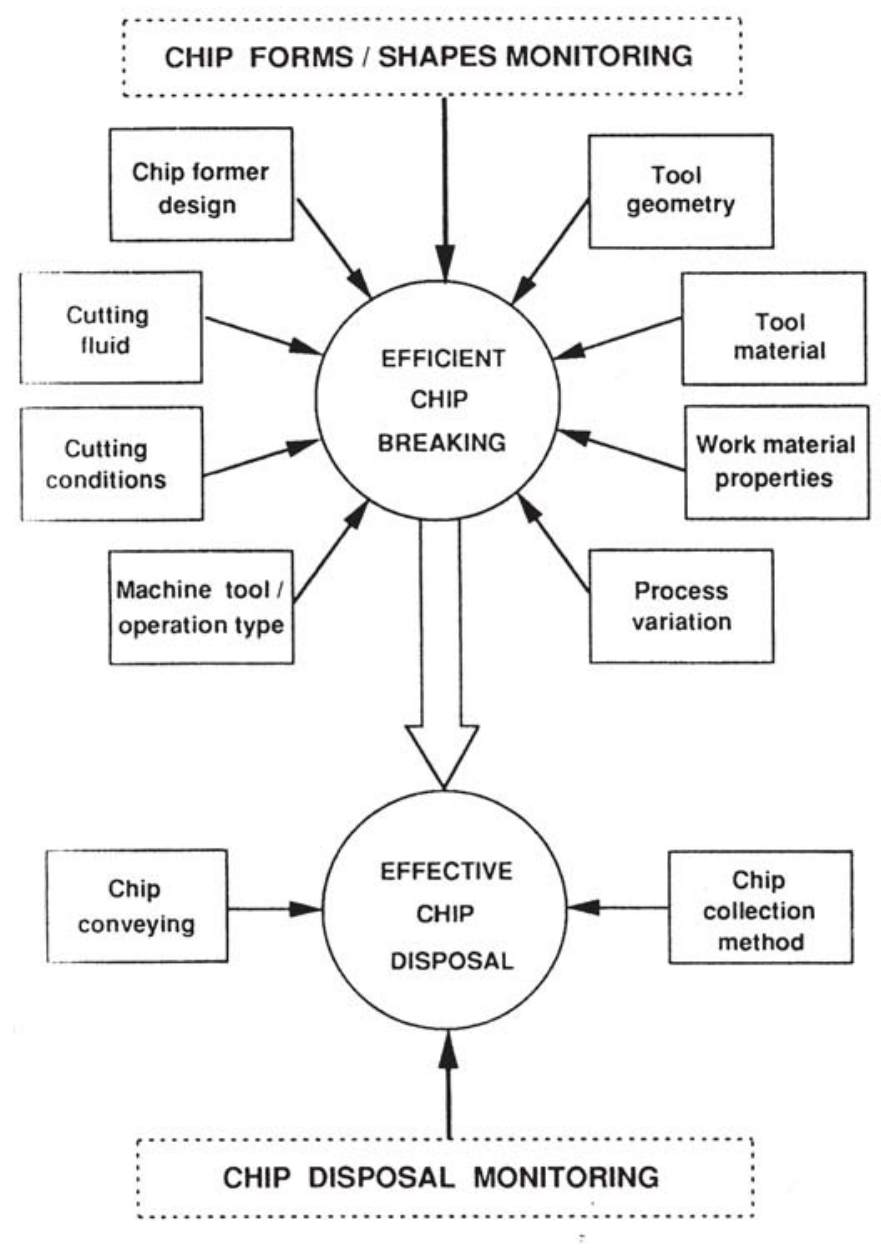

Figure 2.17: The concept of total chip control. (Jawahir and van Luttervelt, 1993)

Type 3 chips, chip control becomes difficult due to the long, continuous, unbroken chip that must be contained. Failure to break the chip results in tool breakage or chip entanglement, both of which result in machining downtime and loss of productivity. Aluminum, low and medium carbon steel are prime examples of materials that produce difficult to break chips.

Even within the class of ductile materials, relative workpiece brittleness - either by alloying or temperature effects - is a major consideration in inducing breakage (Nakaya- 
ma and Arai, 1977). The issue of chip control characterization with a materials perspective has not been treated adequately (Jawahir and van Luttervelt, 1993). von Turkovich (1981) remarks that the fields of metallurgy and metal cutting research have typically excluded each other. However, it would be fair to assume that one of the primary reasons for the lack of information is due to the wide variety of work materials available for machining. The problem exacerbates when one considers the chip variants produced even with small changes in heat treats and composition (Allen, 1969). Some material aspects of chip breakability from Nakayama's original work (Jawahir and van Luttervelt, 1993) are illustrated in Figure 2.18.

Irrespective of the work material consideration, Nakayama (1962) classified chip breaking mechanisms into three broad categories as shown in Figure 2.19. In each case, chip breakage occurs due to the bending strain induced in the chip cross-section when the free end of the chip anchors itself to an external obstruction, mainly the workpiece sur-

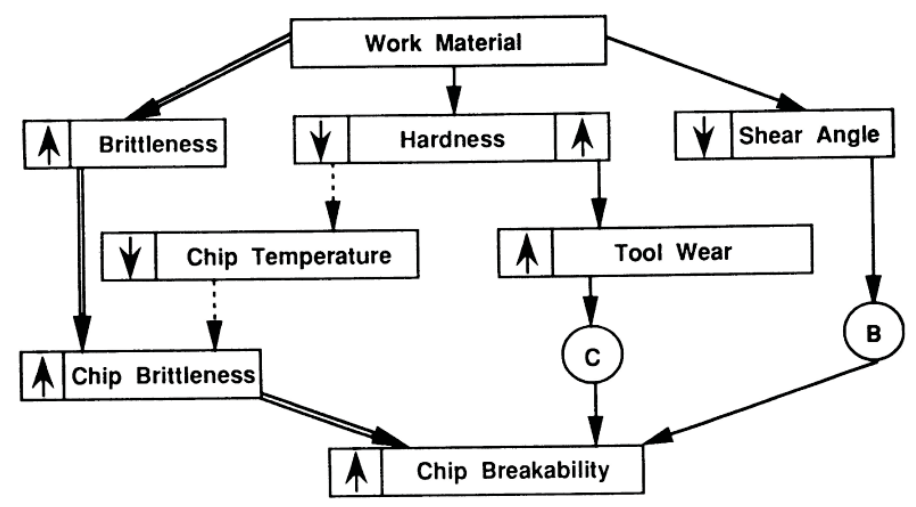

Figure 2.18: Nakayama's work material considerations for chip breakage. (Jawahir and van Luttervelt, 1993) 


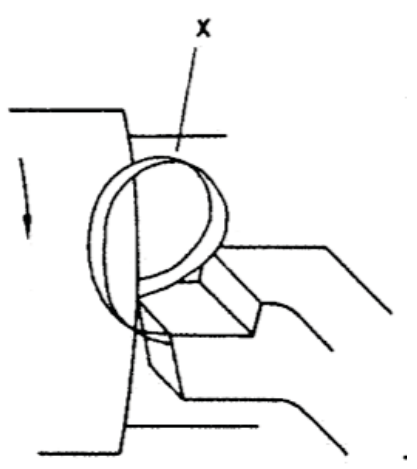

(a)

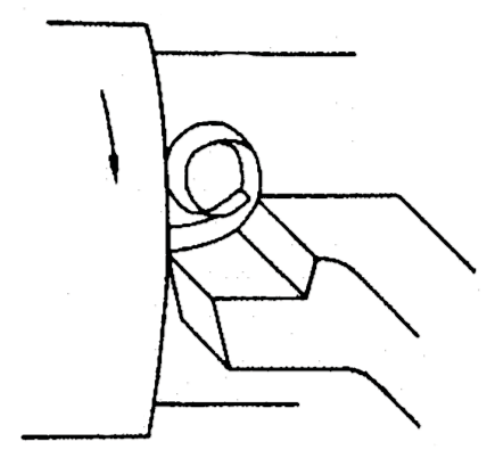

(b)

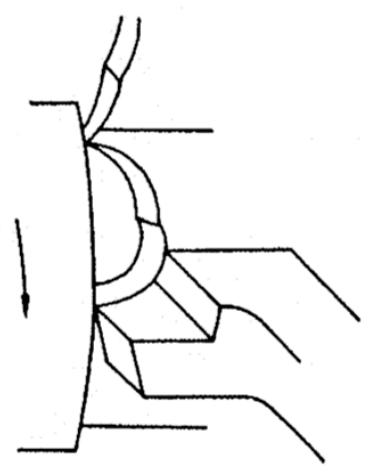

(c)

Figure 2.19: Different modes of chip breaking proposed by Nakayama. (Worthington and Redford, 1973)

face or the tool flank. A similar mechanism occurs if the chip meets other obstructions such as the tool post. When the bending strain so induced in the chip exceeds the ultimate strain of the chip material, the chip will rupture or break. Nakayama also presented a chip breakage criterion which is given by

$$
\epsilon_{\mathrm{b}}=\frac{\mathrm{h}}{2}\left(\frac{1}{\mathrm{r}_{\mathrm{o}}}-\frac{1}{\mathrm{r}_{\mathrm{f}}}\right)
$$


where $\varepsilon_{\mathrm{b}}$ is the bending strain in the chip, $\mathrm{h}$ is the uncut chip thickness, $\mathrm{r}_{\mathrm{o}}$ is the initial chip curl radius and $\mathrm{r}_{\mathrm{f}}$ is the final radius of chip curl before fracture.

Experimental studies of chip breakage are possible with either high-speed photography or cine techniques (Kennametal, 1984). Using high-speed filming techniques, Spaans and van Geel (1970) studied the mechanics of each of the chip breakage modes proposed by Nakayama. They note that the chip breakage is strongly influenced by the "history" of the preceding chip breaking mechanism. In a more recent work based on the effects of the restricted contact tool on chip breakage it was shown that chip breakage is highly cyclic in nature (Jawahir, 1990).

Various techniques for breaking long continuous chips are reported in the literature. Due to its practicality, the most prevalent is the use of chip breakers in conjunction with the cutting tool. Chip breakers function by obstructing the natural flow of the chips. Clearly a misnomer, chip breakers in reality do not actually break the chips. Rather, they serve to deflect the chip (from its natural path) towards other barriers such as tool flank or the rotating workpiece (Henriksen, 1954). Upon encountering an obstruction, the chip breaks in accordance with the limiting bending strain theory proposed by Nakayama. Figure 2.20 shows the action of chip breakers during machining.

One of the most simple chip breaker designs was that proposed by Klopstock (1925), where chip breakage was induced by an external obstruction provided directly on the ground away rake face of the tool. By eliminating a part of the rake face Klopstock aimed to reduce friction and also keep the chip flow along a predictable path. Providing an obstruction, either built in or a separate shim piece, along the chip flow path forced the chip to curl back towards the rotating workpiece and break. The complete tool assembly 


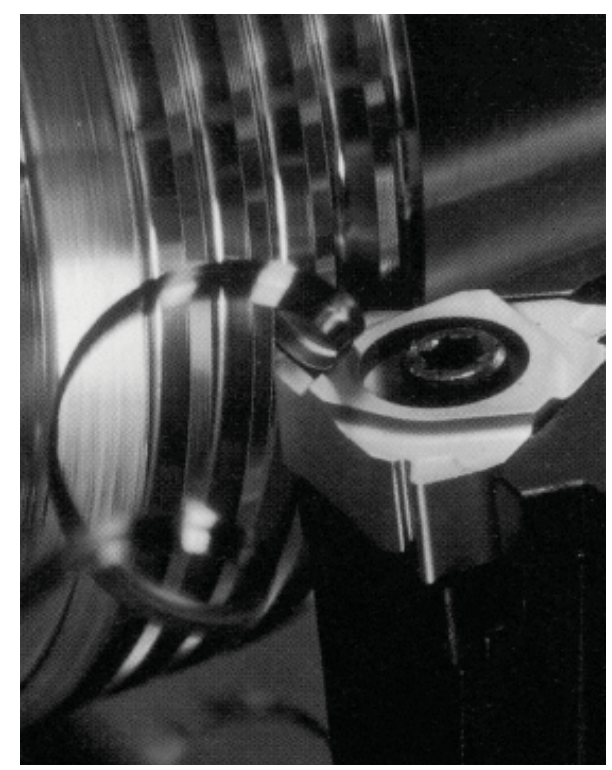

Figure 2.20: Chip breaker action shown for a threading operation.

(Courtesy of Sandvik Coromant, 1996)

(ground tool rake face and chip breaker) is sometimes referred to as the "Klopstock mechanism" in the literature. Klopstock made no mention on the exact settings for the chip breaker. Neither the height nor the placement of the breaker unit was specified. Clearly, incorrect use of a clamp-on chip breaker could cause chip jamming or, if used without adequate design considerations, it ceased to be effective (Henriksen, 1955).

An improvement on the early clamp-on chip breaker design was the use of molded chip breakers where the breaker design was sintered directly on the tool rake face to serve the same purpose as the separate clamp-on piece. Again, the primary design consideration is to force the chip to deviate from its flow and induce breakage. Since machining is a dynamic process, the molded one-piece tool design entails an optimal geometry and position for breaking chips across a range of cutting conditions. Henriksen (1954) analyzed 
and classified the chip breaker types based on the most effective designs. Optimal machining conditions were laid out for each of the designs tested. What is interesting in Henriksen's work is that he arrived at detailed process parameter charts that served as a simple chip breaker reference for optimum broken chips. Lutov (1962) elucidates the methodology for selecting the optimum chip breaking grooves based on cutting parameters. Wirfelt (1969) articulates the pros and cons of different 3D chip breaker designs. Many other references are also available on design and selection of the right chip breaker geometries. In fact, a specific industry standard, ANSI B212.20-1980 (R-2002), covers the dimensional specification and style of chip breakers for carbide inserts. However, the different geometric shapes used in breakers are not covered within the scope of this standard.

With the proliferation of disposable inserts, chip breaker designs have become complex and can handle a range of 3D chip forms (Nakatani, 1973). Modern inserts designs are based on both work material and process conditions. The primary design consideration is accessibility of the machined part geometry (Destefani, 2002). To optimize the large variety of work materials in use, the present work material taxonomy groups similar work materials based on their machinability and properties (Eastman, 1999). Current standards define work materials into six broad groups: P, M, K, N, S and H type. Tool materials are designed to address specific properties of each material group (Schneider, 2002). Each tooling group (for specific material classes) is further divided into subgroups based on cutting parameters: rough machining, medium machining and finish machining (Sandvik Coromant, 2009b). Separation of the cutting conditions into specific ranges helps optimize the tool selection process. Chip breaker designs can then be fo- 
cused on a specific cutting condition. A cursory look at an industrial catalog (Sandvik, 2009a) reveals specific designs addressing individual material and cutting conditions. These designs take on many shapes such as grooves, bumps, dimples, serrations, etc. as shown in Figure 2.21.

Cutting speed, feed and depth of cut (Tungaloy, 2010) are the three basic variables affecting chip breaker designs. The latter two are the primary considerations in chip breaker designs since they define the cross sectional area (Nakayama and Arai, 1980). In fact, chip breakers are designed for specific combinations of these two cutting parameters. Since the feed is equivalent to the uncut chip thickness and the uncut chip thickness

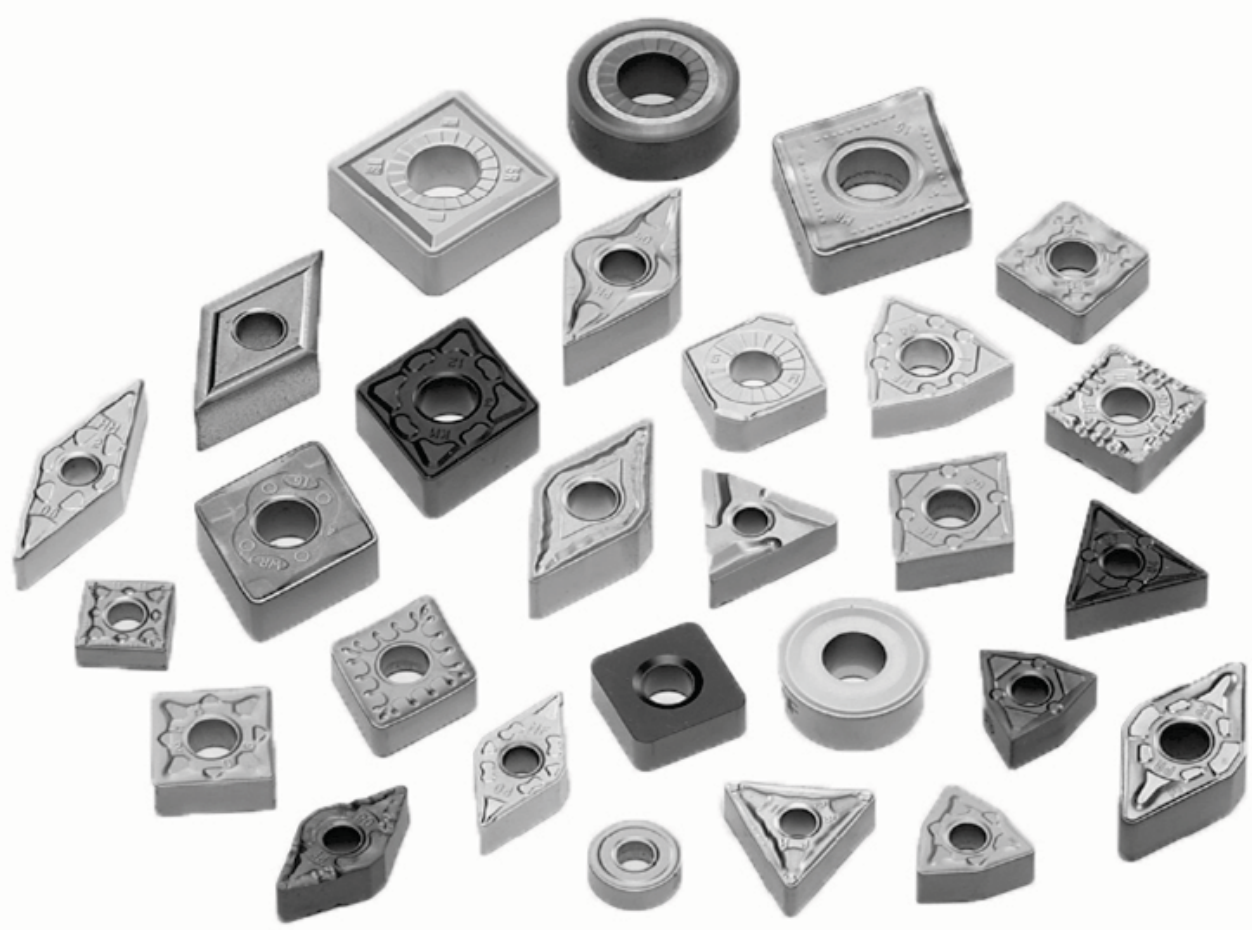

Figure 2.21: A sampling of chip breaker designs available. (Courtesy of Sandvik Coromant, 1996) 
decides the breakability of a chip, increasing the feed rate is usually the first recommendation to induce breakage in difficult to break chip conditions (Sandvik, 1996; Tungaloy, 2010). A general representation of chip form as a function of feed and depth combination is shown in Figure 2.22.

At present, designing chip breakers involves heuristic practices to arrive at an optimum design (Jawahir and Oxley, 1988; Katbi, 1998). The use of a particular chip breaker does not necessarily guarantee adequate chip breakage but rather serves as a good first step to start cutting trials. Most chip breaker designs work well over a specific range of cutting parameter values. Sometimes minor deviations can cause a change in the chip form and reduce the breaking capability of the chip. One obvious challenge in chip breaker design is maintaining the chip trajectory so that it can be consistently deflected to the "anchor" points - the tool flank or work shoulder - in order to break it. Highlighting this issue, Spaans (1970) presented a critical value called the Lead-Width Ratio (LWR) to

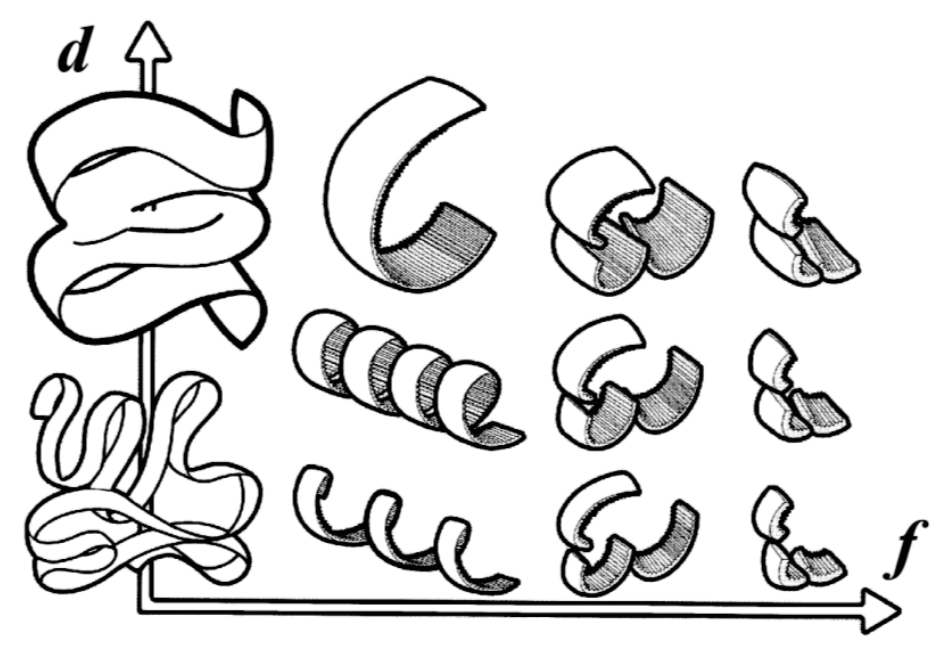

Figure 2.22: Generalized representation of chip forms shown as a function of feed and depth of cut. (Courtesy of Sandvik Coromant, 1996) 
identify the condition for non-breakability of the chip. The LWR value is a function of chip curl, depth of cut, lead angle and chip side flow angle. Spaans found that a long coiled unbroken chip forms at a critical value LWR $>2$ and this chip tends to flow on the tool shoulder without meeting any of the obstacles involved in breakage. Jawahir (1988c) tested an extensive range of chip breaker designs available in the industry and presented chip breaker effectiveness via chip breakage charts. His results indicate that the chip breakability varies considerably based on cutting condition, breaker designs, coatings and tool geometry. These results are quite similar to that reported by Nakayama and Arai (1980), who found that the breakability of the chip changed drastically by merely changing the corner radius or by introducing grooves perpendicular to the cutting edge.

Another aspect of chip breakage is configuring the right size for broken chips so that they may be easily disposed. Obviously, chips can have different sizes when they do break. The term "acceptable" chip breakage is itself ambiguous in the study of chip control. No definitive guide exists on what constitutes a good chip or a bad chip. A qualitative assessment is included in the machinability standard ISO 3685-1993. However, this guidance serves more as a comparison chart for reporting machinability than for making an informed decision on chip breakability. Registering chip morphology has been withdrawn in reporting machinability tests. Researchers (Henriksen, 1954; Kluft et al, 1979; Jawahir and van Luttervelt, 1993) in the past have suggested on what constitutes good and acceptable chip forms. Other sources that list acceptable chip forms are also available (Boothroyd and Knight, 2006). Kluft's proposed recommendation is shown in Figure 2.23. 


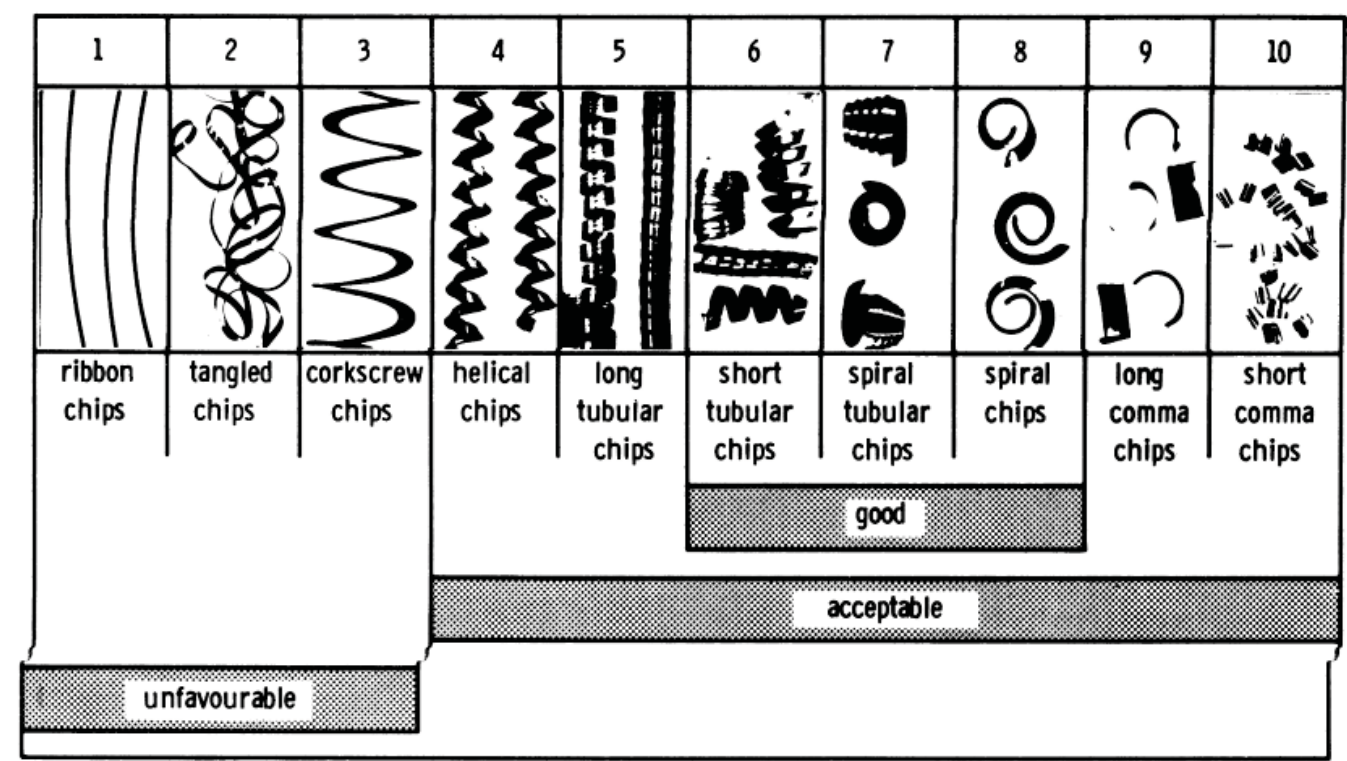

Figure 2.23: Kluft's recommendation for acceptable chip forms. (Kluft et al., 1979)

Industry product catalogs (Tungaloy, 2010) also serve as good resources for acceptable chip forms. In all of the cited sources, "desirable" or "good" chips are in effect defined as short, segmented chips that are easily evacuated without entanglement. No definitive quantitative measures are available to evaluate acceptable chip forms. Infrequently, terms such as Chip Packing Ratio (CPR), Chip Packing Density (CPD) or the Chip Packing Density Index (CPDI) are used as a quantitative metric to classify "good" chip forms (Shaw, 2005; Zhang, 1994; Machado and Wallbank, 1994). The chip packing ratio, $\mathrm{CPR}$, is defined as the ratio of the cut material to the uncut material (Shaw, 2005). Zhang (1995) defines the CPDI as the ratio of 1000 times the chip packing density, CPD, to the cut chip material density, CMD. Tabulated values of the CPDI for various chip forms from Zhang's work are as shown in Figure 2.24. 


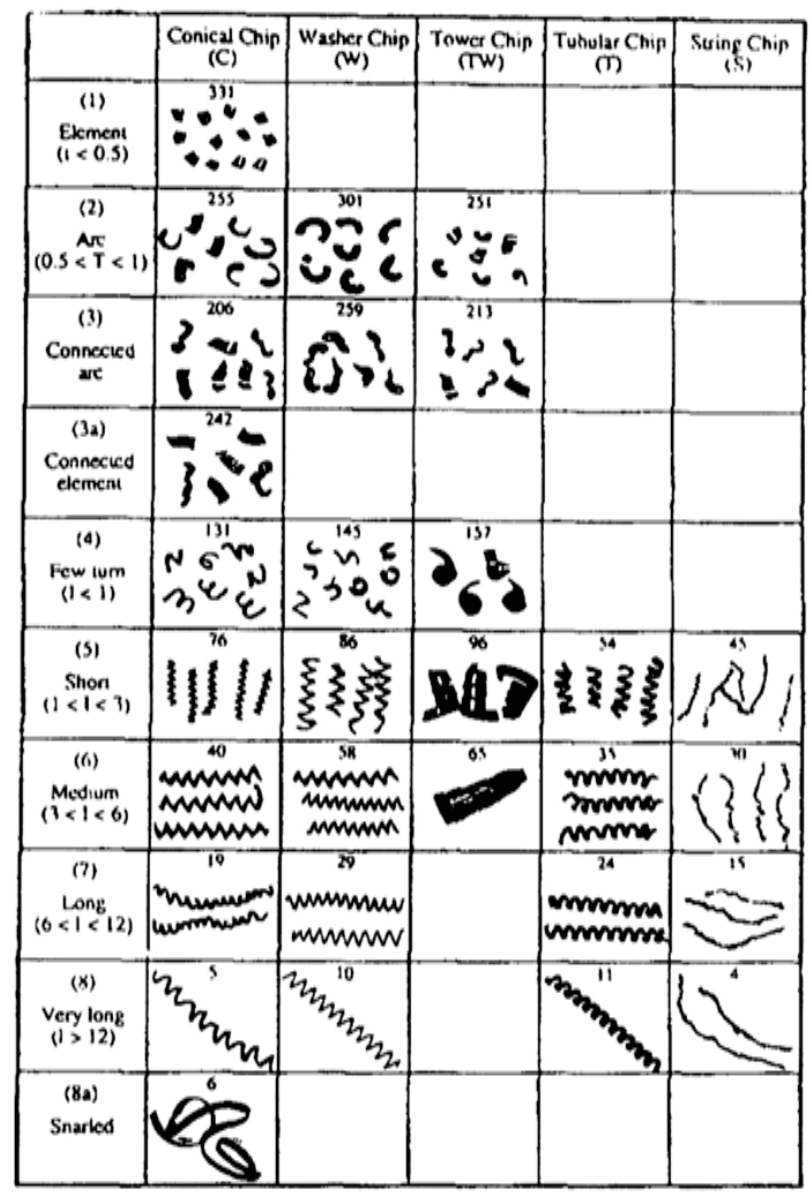

Noc: $1 . .$. Iurn, 1 .... length $(\mathrm{cm})$

Figure 2.24: Tabulated chip packing density index. (Zhang et al., 1995)

One immediate drawback observed in the CPDI method is its inability to indicate the detrimental effects of chips to the process economics. Chip breakage efficiency is based only on the shape of chips collected in the chip bin. In the CPDI table, compared to the short tubular chips (row 5, column T), the tower chip with few turns (row 4, column TW) obtains a higher CPDI rating. Experimental results however, indicate that the latter chip type tends to increase tool crater wear rates due to the tight curls and are generally deemed not acceptable (Jones and McCreery, 1973; Tungaloy, 2010; Grzesik, 1997). 
Such tightly curled chips are also called over broken chips (Okushima et al., 1960; Henriksen, 1954) as against the under broken chips that are the long unbroken variety.

Other notable attempts to quantify chip breakage includes the work of Li and Rong (1999) who formulated a critical value for feed and depth of cut that define the chip breaking limits. The critical feed/depth values serve as demarcation between the breakable and unbreakable chip regions. In another innovative approach, Fei and Jawahir (1994) used fuzzy logic to assign weighting factors for chip breakability. Balaji et al. (2006) provide a useful review assessment on chip forms and breakability.

Apart from chip breakers, other novel chip breaking techniques have also been proposed. A very common practice is to use a blast of high-pressure air to evacuate chips from the cutting area (SME, 2001). At high feeds and depths, however, increased chip stiffness due to the larger chip area more or less negates the air blast effect. Highpressure coolant (>2000psi) can be substituted for air in such conditions (Machado and Wallbank, 1994). More recently, high-pressure coolant application to the chip root has gained widespread application in the industry (SECO Tools, 2010; Sandvik, 2009a). By reducing the tool-chip contact length and simultaneously generating an up-curl, the highpressure coolant method serves to break the chip against the workpiece. Recommended usage of the high pressure tooling system has been for adhesive materials such as Titanium. One present limitation to this method is the lack of high-pressure systems in general machine tools due to added costs.

Use of cryogenic coolants has also been reported (Christopher, 1990; Hong et al., 1999) to alleviate chip breakage problems. In fact, ready-to-use commercial cryogenic systems - the ICEFLY from Hardinge., Inc - have been a recent development in this area 
(Hardinge, 2010). The main factor that seems to limit this promising technology is the storage of high-pressure cryogen coolants. Application of cryogenic coolants in machine tools requires specialized design and safety considerations. Another unique technique reported has been the use of directed lasers to break chips (Li and Xie, 2001). Chip breakage is achieved by focusing the laser beam to the chip on the rake face. However, this technology does not consider the highly unstable nature of chip flow. A more practical application of the laser technique has also been reported (Americhip, LLC, 2010). Chip breaking is achieved by scribing a groove ahead of the tool during the cutting process. However, no data are available on its exact application and characteristics.

Vibration assisted machining is yet another proposed alternative method (Danielyan and Gritsaenko, 1962) for inducing chip breaking. In its application, chip breakage is achieved by controlled oscillations of the tool in the direction of feed. A variation of this method is to combine a tilt or rotational oscillation with the longitudinal oscillation motion (Shinozaki and Hirota, 1970). Smith et al. (2009) recently reported on a study using oscillations in the $\mathrm{CNC}$ tool path to generate chip breaking. While the results indicate reduced temperature at the cutting edge, they acknowledge the possible detrimental effects of tool vibrations on surface finish, which was not a subject of their study. The problem with vibration assisted machining and surface finish has been highlighted very early (Ostwald, 1967). In a survey on vibratory machining for chip breakage, Skelton and Tobias (1962) note that vibratory machining and surface finish seem to be at odds with each other. They conclude that favorable conditions for chip breaking (low frequency, large amplitude) results in bad surface finish while the unfavorable conditions for chip breaking (large frequency, low amplitude) cannot break chips efficiently. Another aspect of 
the vibratory method of chip breakage is the complexity involved in matching the cutting frequencies and amplitude with the chip breaking frequency and surface finish (Spaans and Hovinga, 1971).

A relatively new technique gaining increasing popularity has been the use of computational methods such as Finite Element Analysis (FEA) to study metal cutting (Strenkowski and Caroll, 1987; Dixit and Dixit, 2008). As with analytical and experimental methods, FEA formulations have also been applied to study the chip formation and flow problem (Strenkowski and Moon, 1990; Sekhon and Chenot, 1993; Arrazola et al., 2005; Marusich et al., 2002). Computational methods offer a major advantage by significantly reducing the number of expensive experimental iterations. Parametric studies of the machining process are possible in a faster and more economical fashion. However, the formulation of the FEA technique generally assumes steady state chip flow and does not account for transients in the chip behavior over its flow path. Chip breakage is formulated as a direct function of the material properties (limiting stresses for breakage) in the shear zone. The main reason is that modeling the entire range of geometry and mechanics of the chip flow evolves into a very complex and cumbersome process due to the large number of variables with no unique boundary conditions. "Total chip control" then reverts to experimental methods to match the chip breakers to process conditions. Nevertheless, when used in conjunction with specific machining process conditions FEA methods provide a significant advantage in understanding the process characteristics. Many modern textbooks on machining theory now include good references on using FEA techniques in modeling the machining process (Stephenson and Agapiou, 2006; Grzesik, 2008). In fact, commercial software packages such as 
ADVANTEDGE and DEFORM have found wide acceptance for their sophistication in modeling the cutting process.

\subsection{Concluding Remarks}

Based on a review of the available literature, considerable knowledge exists on the fundamental understanding of chip flow and control. Most analytical works are mechanics based and delve into both orthogonal and oblique modes of cutting. A majority of the body of knowledge available today discretize the more complex $3 \mathrm{D}$ chip problem into a simpler 2D process. Even though this does not accurately depict the true nature of the process, such a methodology obviously leads to better understanding of the complete

process. It is necessary to acknowledge the extreme degree of complexity involved in the modeling of a complex, transient process such as machining. A comment worth noting for its profoundness is due to Dewhurst (1978), who notes that "The orthogonal machining process is not uniquely defined by any given set of steady state conditions.....random disturbances during machining, due to imperfections in the work material for example, are likely to produce a changed steady state". Nevertheless, far more is known today about the basic rules governing the chip behavior to adequately design processes as well as control chip flow. Today, mature chip breaking designs exist to address difficult to break chip types. What is limiting however, is the narrow range over which these techniques operate. Jawahir and Oxley (1988) note that the pressing need is for an "optimum" chip breaker design, or in other words, a universal technique that can address chip breakage in as wide a range of cutting conditions as possible. As such, this research attempts to study a new method to achieving universal chip control. 


\section{CHAPTER 3}

\section{A MODEL FOR UNIVERSAL CHIP CONTROL}

\section{GEOMETRY}

A new technique for chip control is discussed in this chapter. In order to lay the groundwork for this research, the original idea and initial feasibility tests that served as the prime motivating factor are first described. A mechanics based approach is utilized in developing a model to predict the tool top form geometry required to achieve a consistent chip flow pattern across multiple cutting conditions. Finally, the development and considerations thereof for a working prototype are presented.

\subsection{Motivations for Universal Chip Control}

It has been acknowledged that achieving "universal" chip control is the ultimate goal for chip control research (Venuvinod, 1977; Jawahir, 1988). Among the myriad techniques utilized, the most common is the use of "bumps and dimples" (hereon termed chip breakers) on the tool top face geometry. Relative to some of the other innovative methods proposed (see Chapter 2), simplicity and robustness is one reason for their wide acceptance. Chip breakers used on disposable cutting inserts operate by deflecting the chip flow towards an obstruction - the workpiece, tool flank or tool holder - where brea- 
kage occurs in one of the three modes as discussed in Chapter 2. However, given the vagaries of the cutting process, it is nearly impossible to design a truly universal chip breaker design that can effectively handle every possible combination of cutting conditions. As an alternate solution, chip breakers are designed to operate only within specific cutting conditions. Even with the latter approach, chip breaker designs are sometimes rendered ineffective due to two factors: either the intended geometry of the breaker is unutilized by the chip or the targeted obstruction is completely eluded by the chip. The proclivity of the chip to alter its behavior even with seemingly the smallest of disturbances presents a formidable challenge to design a universal chip breaker. In a very interesting display of this effect, Figure 3.1 shows Finnie's (1963) demonstration that a dramatic difference in chip behavior can be obtained by merely touching a chip being formed. Note that the use of a finger to alter chip flow is rather dangerous and unsafe by today's standards.

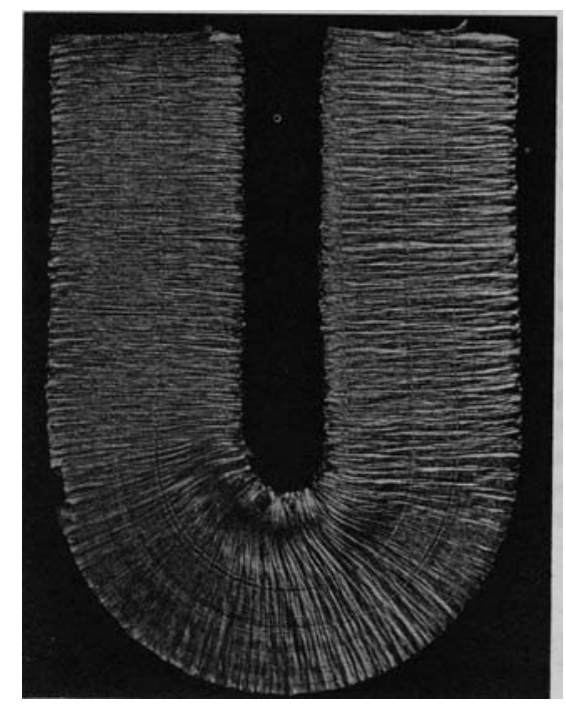

Figure 3.1: Finnie's demonstration of chip flow changes with a minor disturbance. (Finnie, 1963) 
To complicate matters further, the mechanics of breaking a chip poses a different challenge by itself. Fundamentally, chip breakage occurs due to a bending strain induced along the cross section of the chip (Nakayama, 1963). Fracture occurs when this strain exceeds the ultimate strain of the chip. The sequence of events leading up to the development of ultimate strain in the chip follows a series of phases before eventual fracture occurs. Jawahir and Zhang (1995) identify four distinct phases leading up to chip fracture. Initially, the chip flow is unstrained but undergoes "constrained growth" under the action of an obstruction on the free end. A bending moment is generated due to the force exerted by the obstruction on the chip. With increasing growth, the chip experiences an increase in the bending strain until it ruptures creating a new chip. Spaans and van Geel (1975) found that the breakage and subsequent formation of a new chip is a cyclic phenomenon.

In analyzing breakage of chips, the defining boundary condition is analogous to a simple rectangular beam deflection problem (Nakayama, 1963). The radius of chip curl decides the magnitude of the induced bending moment on the chip. A tighter chip curl radius naturally results in increased chip breakability due to the higher bending stresses induced by the small radius of curl (Nakayama and Arai, 1977). Based on their investigation of the chip formation process using a high-speed camera, Batzer et al. (1997) observed that chip fracture due to the induced bending stress always occurs along the shear band serrations present on the top face of the chip surface (i.e. the air side of the chip). The chip serrations serve as stress concentration zones. Nakayama (1963) reserves comments on the direction of bending and its effect in inducing chip breakage. Based on results from experimental chip bending tests, Nakayama showed that the chip 
breakage occurs at a much lower bending moment and curl radius if subjected to tensile loading of the top rough surface. In short, the breakability of the chip increases under tensile loading (backward bending) rather than compressive loading (forward bending) of the chip's rough top surface. Figure 3.2 shows Nakayama's results for two different chip materials, with $M$ being the induced bending moment and $\theta$ being the angle of deflection corresponding to a specific bending moment. For the sake of clarity, the two conditions forward and backward bending of the chip top surface - are illustrated in Figure 3.3.

From a practical machining perspective, backward bending can be theoretically induced in a chip by presenting an engineered obstruction to the chip flow. The most simple obstruction that could potentially bend the chip backwards is the add-on type of

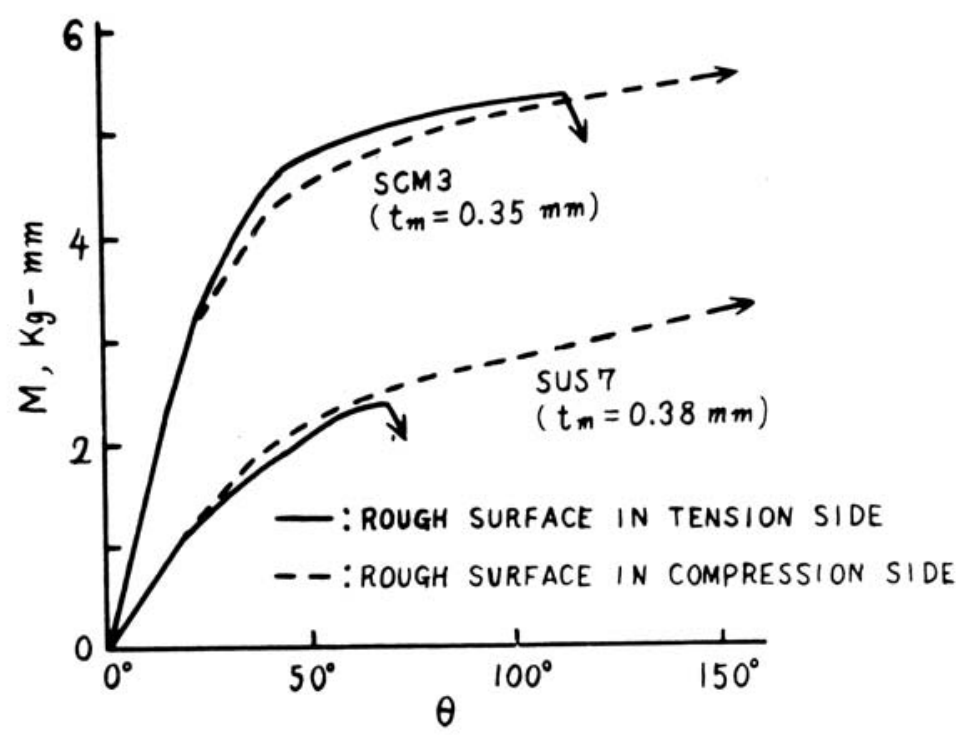

Figure 3.2: Bending moment induced in a chip loaded under tensile and compressive loading of the top rough surface. (Nakayama, 1963) 


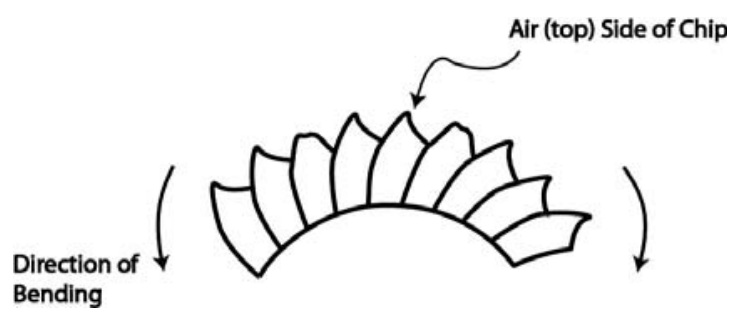

(a)

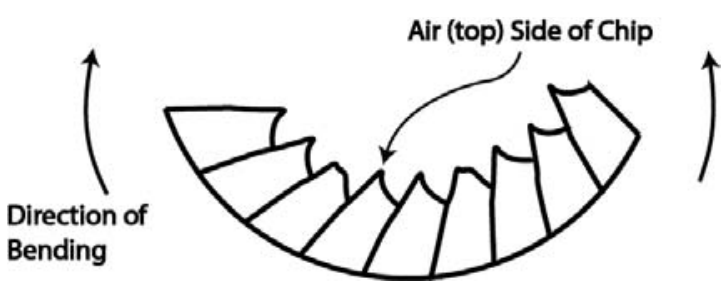

(b)

Figure 3.3: Schematic illustration of (a) Backward bending (b) Forward bending.

chip breaker. Figure 3.4 shows two possibilities where backward bending can occur due to an add-on chip breaker. Note that the images were used by Henriksen (1955) to emphasize detrimental chip breaker designs, but have been used here to provide a visualization of the induced backward bending. Note also that mere addition of an addon chip breaker does not guarantee chip backward bending. As explained by Lutov (1962), the position and design of the add-on chip breaker is crucial. In most cases, the chip may very well - and in fact, does - curl forwards (compressive loading) due to the

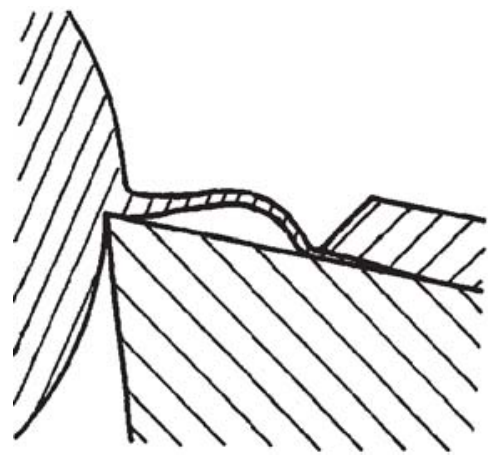

(a)

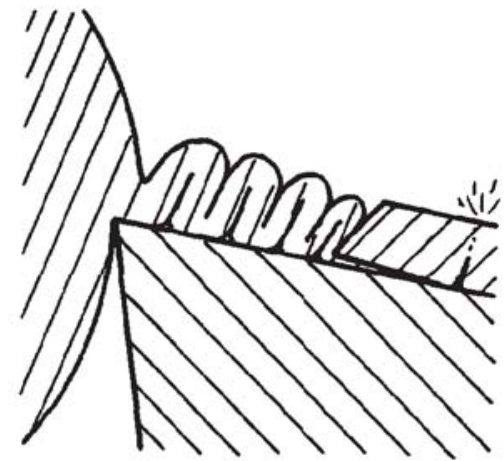

(b)

Figure 3.4: Two possible cases that can result in backward bending. (Henriksen, 1955) 
obstruction thus negating the possibility of backward bending. The possibility of inducing chip backward bending reduces further in disposable inserts with built-in chip breakers. Following Nakayama's (1962) chip breaking modes (see Figure 2.19), backward bending can occur only if the chip end anchors to an obstruction $\underline{A N D}$, the radius of curl is conducive to force backward bending in the chip. Given the randomness of the chip formation process as well as the varying nature of chip curl (Spaans and van Geel, 1970), intentional backward bending of the chip is an impossible task with the standard chip breaker design. From a survey of the literature, it is found that the mode of bending is generally not considered in most chip breakability research. The main reason can be obviously attributed to the difficulty in engineering any particular mode of chip bending.

From the discussion so far, it is obvious that the natural unpredictability of the chip flow and breakage makes present chip breaker designs a highly complicated and nonrepeatable process. Based on Nakayama's (1963) results, it can be inferred that chip backward bending should present a more reliable means to increase chip breakability. The main advantage is the lower force and smaller radius of curl required to break chips under backward bending. But, while backward bending in a static chip is easy, the same does not hold true for the dynamic chip flow process in machining. To test the possibility of inducing backward bending in chips, preliminary feasibility experiments were conducted at Endres Machining Innovations LLC (EMI, 2005) based in Houghton, MI. The explicit objective was to test the reverse chip bending theory in practical machining since no documented work exists on inducing backward bending during the machining process.

EMI's experimental setup used straight edged $\left(\psi_{r}=0^{0}\right)$ free orthogonal cutting thus eliminating the added effects of the corner radius on chip curl and flow. The process 
mimicked cutting of a tube by using a face grooving tool to machine a groove ahead of the tool with the thickness of the lip forming the depth of cut. A mock-up guidance system affixed to the tool holder enabled chip backward bending. Figure 3.5 illustrates the setup from the feasibility experiments. The backward bending tests were performed with
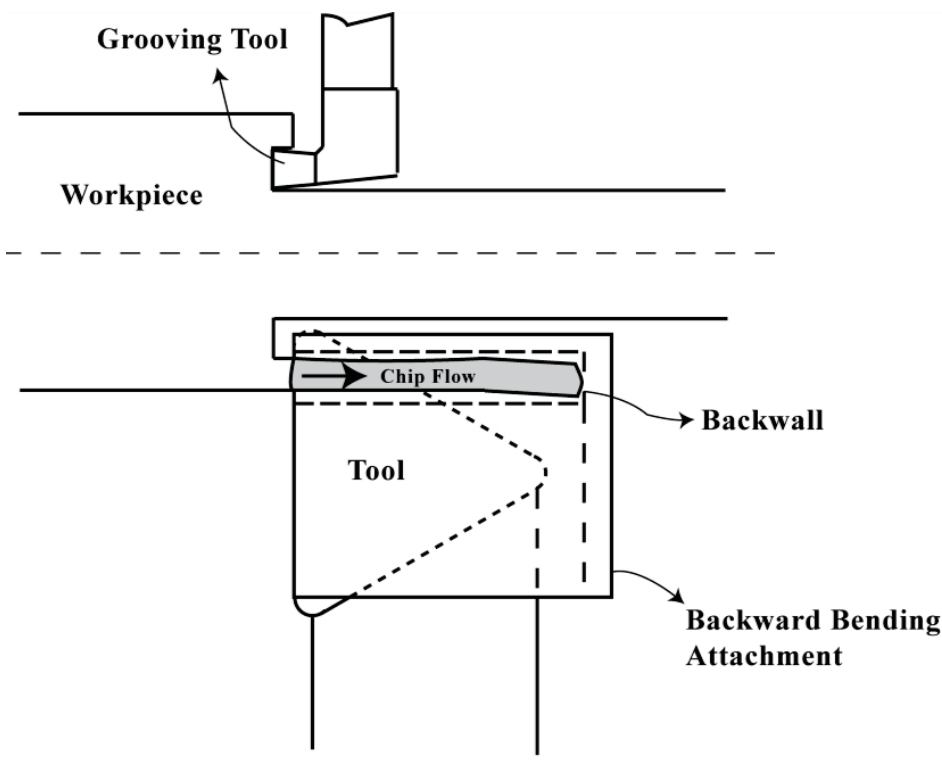

(a)

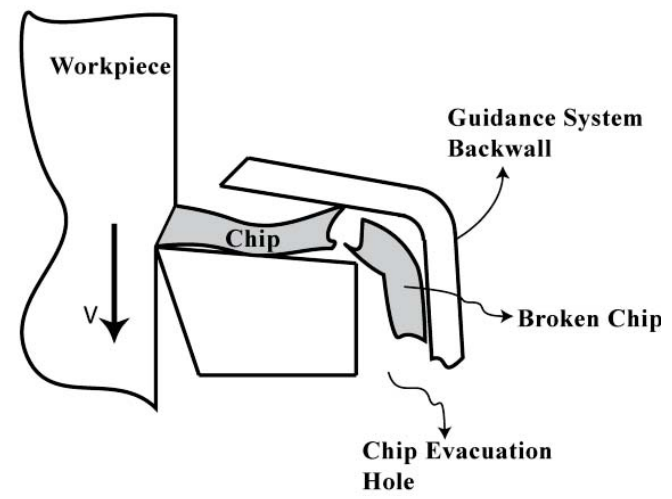

(b)

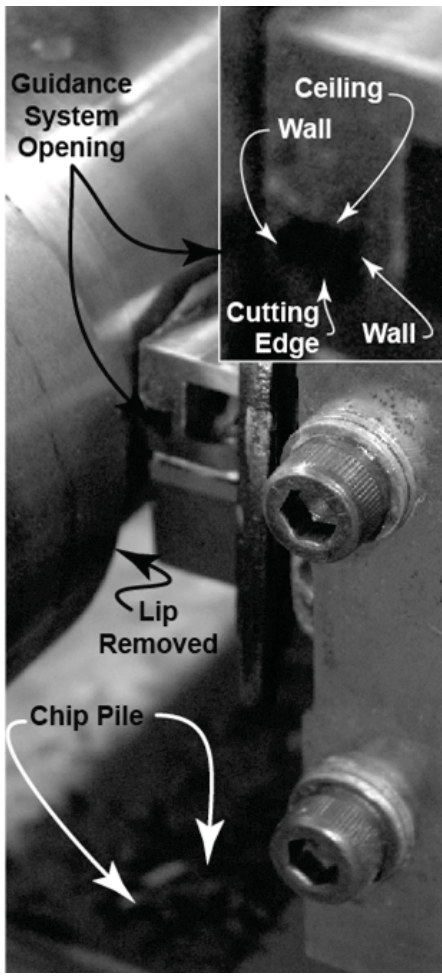

(c)

Figure 3.5: (a)-(b) Illustration of the backward bending feasibility tests (c) Picture of mock up tooling. (Courtesy of EMI, 2005) 
a triangular flat insert with depth of cut, $d=0.05$ inches. As a benchmarking exercise, the chips produced from the cutting tests were compared to those from a CNMG432 insert with a generic chip breaker design. Note that in the case of the benchmark insert, the depth of cut used was higher at $d=0.09$ inches which was the recommended first cut approach. Both tests were run using standard crankshaft steel with the same speed, $\mathrm{V}=250$ sfm and feed, $f=0.008 \mathrm{inch} / \mathrm{rev}$.

Results from the cutting tests at EMI reveal some interesting observations. Since the guidance system in the backward bending cutting test inhibits the up-curl of the chip and bends the chips backwards, the chips produced exhibit straight flat segments with lengths ranging between 0.2 to 0.5 inches. All of the tests on record indicate no instance of chip jamming inside the guidance system. In comparison, cutting tests with the benchmark CNMG432 insert produced up-curled chips, with small acceptable segment lengths. Figure 3.6 shows the chips from both tests - those produced by the guidance system in place and those from the standard CNMG432 insert.

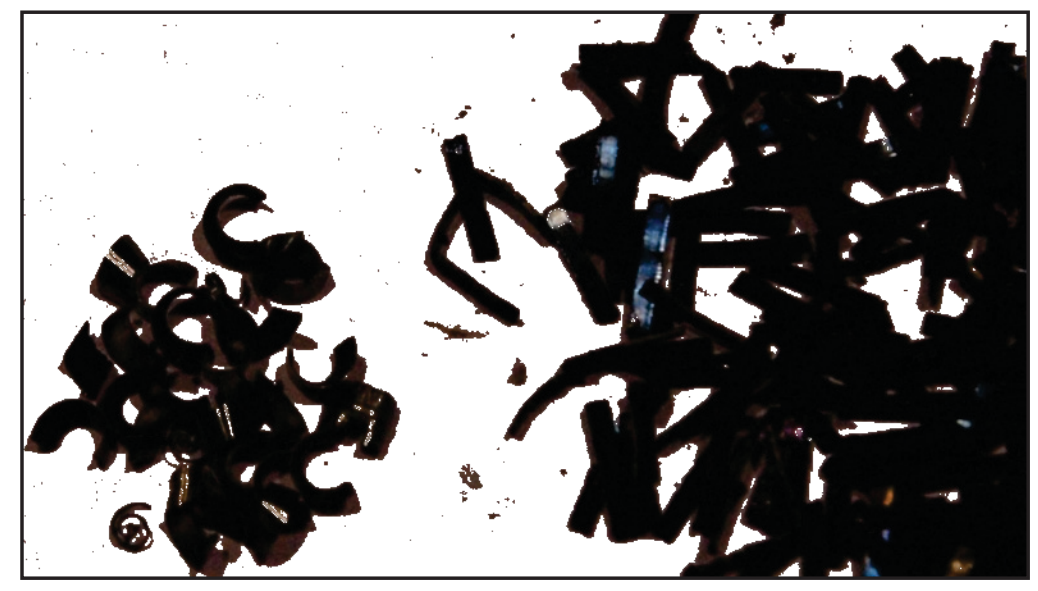

Figure 3.6: Chips obtained from (left) CNMG432 benchmark insert (right) Backward bending tooling. (Courtesy of EMI, 2005) 
As a control test, backward bending experiments were then run with the guidance system removed. The direct consequence was that the chip form changed dramatically from the short straight segments to a long snarled variety. Similarly, when the benchmark CNMG432 insert with chip breaker was used at a lower depth of cut, $\mathrm{d}=0.05$ " (still within the recommended depth of cut) drastic chip form changes were noted; the acceptable short up-curled segments morphed into a long unbroken corkscrew chip variant. Figure 3.7 shows the changes in chip forms under the changed cutting conditions.

The feasibility trials at EMI throw up two relevant (to present research) conclusions:

1) The use of standard chip breaker top form geometry is indeed ineffective in comprehensive chip breakage. Support for this fact comes via the two entirely different chip variations produced with the benchmark insert with chip breakers at two different depth of cut conditions - acceptable chips at a higher depth of cut $(0.09$ inches) and unacceptable chips at a lower depth of cut (0.05 inches). Results from

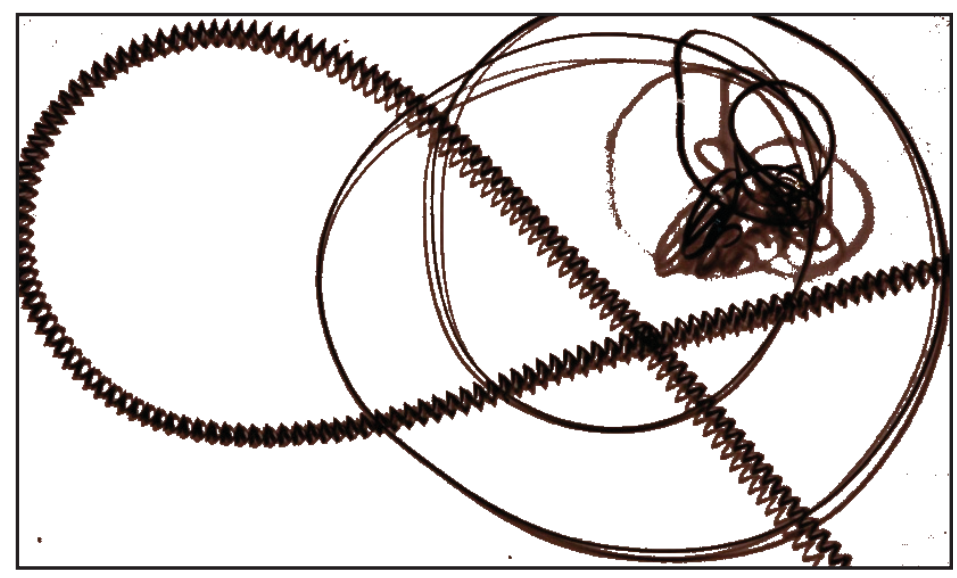

Figure 3.7: Change in chip forms as noted for (left) Helical coiled chip with the benchmark CNMG432 insert at a lower depth of cut and (right) Straight snarled chip with the tool design for chip backward bending without the top guidance unit. (Courtesy of EMI, 2005) 
past research (Henriksen, 1964; Jawahir, 1987) also corroborate this conclusion.

2) If backward bending of the chip is indeed possible in practice, it can result in consistent, acceptable chip forms.

The conclusions listed then also raise some valid questions:

1) Could practical machining involving non-free oblique cutting, i.e. with a corner radius (see APPENDIX A), utilize the backward bending chip breaker technique for truly Universal Chip Control?

2) Given that initial EMI trials used single straight edged orthogonal cutting where chip flow is rather easy to predict, is it possible to preemptively direct the chip flow towards a "target" and induce a backward bending mechanism when operating under non-free oblique machining conditions?

Achieving backward bending for chip breakage under non-free oblique cutting conditions naturally involves providing a means of consistently directing the chip to a prespecified target location. Most research on chip control in non-free oblique cutting attempt to predict the chip behavior a posteriori under specific geometry and cutting conditions. Few works if any have been directed at pre-emptively controlling the chip flow. Only two notable attempts have been identified from the published literature. Nakayama et al. (1981) suggest introducing a spherical rake face groove to force the chip into a tighter side-curl. More recently, a similar scheme was also proposed by Fang et al. (1997), who use a helical groove on the rake face to achieve a similar end. Currently, a few manufacturers use similar (to that of Nakayama et al., 1981) designs by providing a curled 3D chip breaker design on inserts for specifically machining steel though, strangely, the design is not widespread. 
With the cutting test at EMI serving as a motivation, this research undertakes a study on the feasibility of a Universal Chip Control system using the backward bending approach for chip breakage. To achieve a predefined chip flow path in oblique cutting conditions, a mechanics based model incorporating a variable cutting-edge inclination angle is developed to guide the chip to a specific target location where it may be captured and broken with a special purpose tool system design as will be discussed subsequently in Chapter 5. Compared to some of the past work on chip control, the model is purely geometry driven and does not use cutting force measurement to predict chip flow.

\subsection{Development of a Model for Tool Top Face Geometry}

In developing the model, the methodology used is similar to that adopted by Okushima and Minato (1959) in that the cutting edge is comprised of a number of small linear segments. The final chip side-flow angle is presumed to be a cumulative manifestation of the geometric orientation of individual segmental lengths.

As a starting point, a flat faced tool $\left(\lambda=0^{0}\right)$ geometry is considered. The initial assumption is that the elemental chip side-flow vector is normal to the individual segment. In essence, this assumption is somewhat similar in nature to Colwell's method of predicting chip side-flow angle but with the elemental segments serving as local equivalent cutting edges. Recall that the classical Colwell's method for predicting chip side-flow angle considers the projected area of cut (or, depth of cut-feed pairing) to develop an equivalent cutting edge. Chip side-flow is considered normal to this equivalent cutting edge. An increase in the depth of cut results in a decrease in chip side-flow value while increasing the feed rate leads to an increase in the side-flow angle. By contrast, in the 
model, the value for the uncut chip thickness, or feed, is set at zero $(f=0)$. Doing so represents a lower bound approach to Colwell's model on the effect of feed on chip sideflow. For any values of $f>0$, it can then be expected that the side-flow incurs an increase in value. Hypothetically, since $f>0$ for all practical machining conditions, it should be expected that the model setting will cause the chip to overshoot the target when implemented. However, since the main objective is to address a universal range of feed and depth conditions and not confine the design to a particular value nor range, the process of using $f=0$ as a starting point is reasonable and justified.

The second important assumption is that Stabler's rule is satisfied for each elemental segment. While some debate exists (Stabler, 1964; Zorev, 1966; Brown and Armarego, 1964; Shaw et al., 1952) on the validity of the rule per se, the present work presumes that the extremely small segmental lengths mandate that the rule is acceptable. A similar approach was also adopted by others - notably, Hu et al.,1985; Young et al. 1987; Wang and Mathew, 1995; Arsecularatne et al., 1994, all of whom propose an equivalent tool edge (ET) approach - to explain past inconsistencies of Stabler's rule.

Based on the two assumptions noted, the required elemental inclination angle is equal to the difference between the assumed natural chip side-flow angle and that needed for the chip element to reach the target location.

For practical considerations, the model conforms to standardized tooling geometry for disposable inserts and is applicable to any of the commonly available insert configurations, i.e. S, C, D, T or V type geometries (Sandvik, 2010). Precluded from the list are infrequently used insert geometries such as R and W, although the latter may well be in- 
cluded. All nomenclature discussed pertain to the ISO recommended system of working planes for a tool-in-use system.

As shown in Figure 3.8, the geometric features of the tool edge can be represented as a series of $(X, Y, Z)$ coordinates in a cartesian coordinate system, called the insert coordinate system hereon. To make judicious use of geometrical symmetry, the coordinate system is defined at the center of the inscribed circle, $i C$. In general, since side flow is dictated by the orientation and engagement of the cutting edge, the coordinate system also needs to be defined relative to the cutting edge orientation. Hence, the insert coordinate system is defined with the abscissa and ordinate being aligned with the axes of symmetry of the insert. Any change in insert orientation (varying tool lead angles, $\psi_{r}$ ) likewise results in a reorientation of the insert coordinate system as shown in Figure 3.8(b). Note that the insert coordinate system can very well be defined in the global machine coordinate system. However, defining the system locally on the tool geometry makes for an easier derivation. Simple coordinate transformation functions can be used to transfer the insert local coordinates to the global machine coordinates and vice versa.

A second coordinate system defines the corner radius region of the insert and is designated the tool corner coordinate system. The tool corner coordinate system is set at the center of the blended curve defining the insert corner radius. This second coordinate system is in-turn referenced within the insert coordinate system located at the center of the inscribed circle. For convenience, suitable subscripts are employed in order to differentiate the geometrical features between various coordinates. All features in the insert coordinate system are designated with the subscript $i$ while the subscript $l$ denotes features in the tool corner coordinate system. For example, the ordered triplet $\left(X_{i} Y_{i} Z_{i}\right)$ 


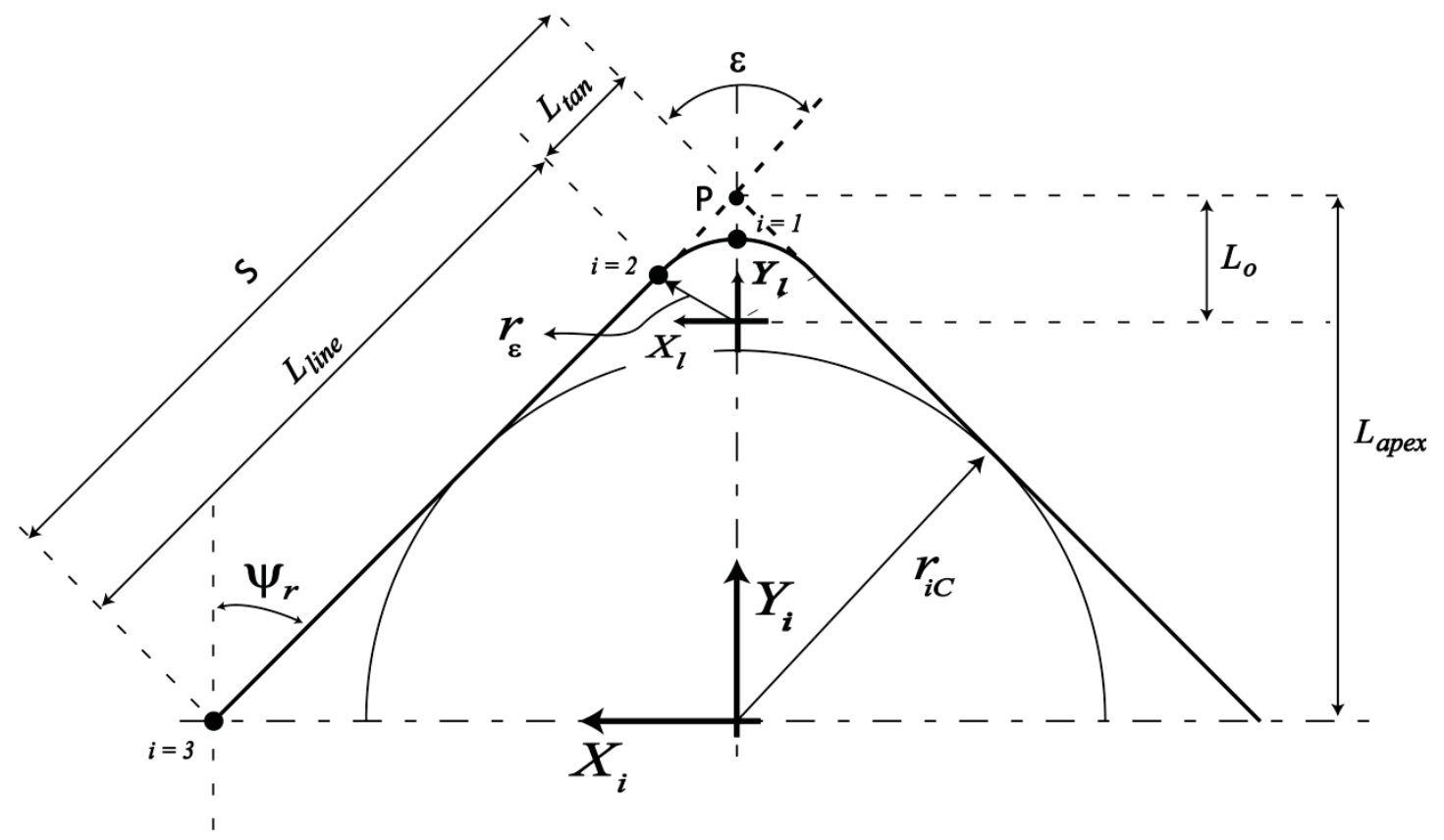

(a)

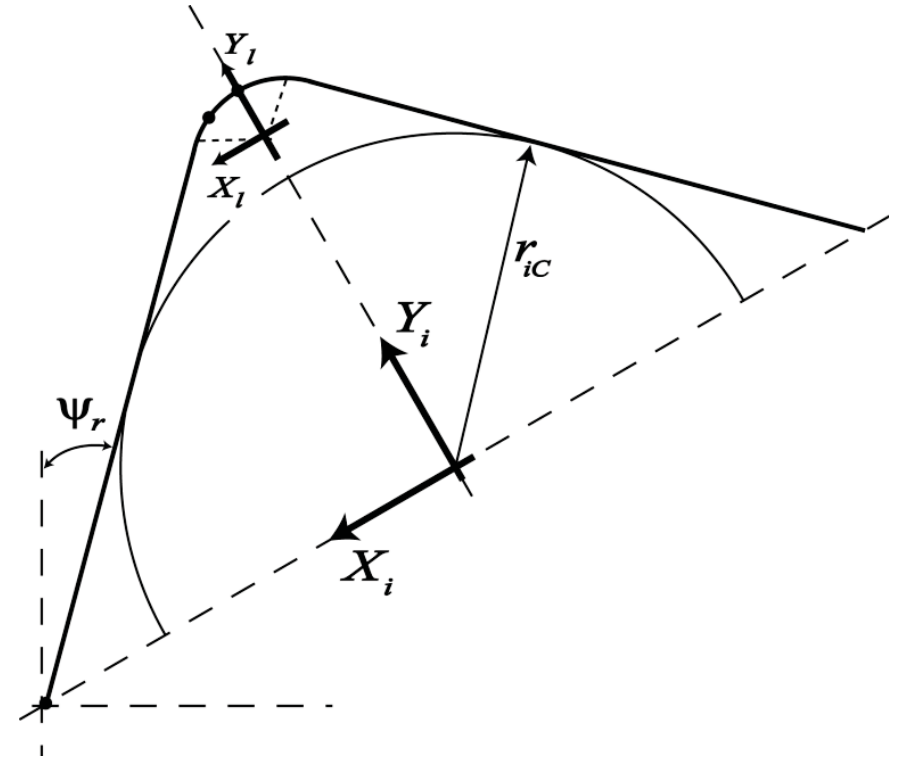

(b)

$X_{i} Y_{i}$ - Insert coordinate system

$r_{i C}$ - Insribed circle radius

$X_{l} Y_{l}$ - Corner radius coordinate system

$r_{\varepsilon}$ - Corner nose radius

$i(=1,2,3 \ldots)-$ Number of points defining cutting edge

$\varepsilon \quad$ - Included angle of insert

$\psi_{r}$ - Lead angle

Figure 3.8: Model setup showing coordinate systems and basic nomenclature. 
indicates that the features are defined in the insert coordinate system. For presentation purposes here, the discussion here is confined to an insert with a square geometry (Stype) although the methodology can be applied to any insert shape.

Definition of the boundaries enclosing the tool geometry forms the essential first step. The only input variables required are the included angle of the insert, $\varepsilon$, and the diameter of the inscribed circle, $i C$; both are straightforward since the basic insert geometry is known. Since the insert coordinates are set up locally and follow the insert orientation, no special consideration need be made for any specific lead angle configuration. The lead angle $\psi_{r}$ can be obtained from the included angle of the insert $\left(\psi_{r}=\varepsilon / 2\right)$. By default, the model considers a neutral tool configuration.

The cutting edge of the insert can be demarcated into a straight-line region and an arc region that defines the insert corner radius. From the geometry of a flat faced $\left(\lambda=0^{0}\right)$ tool insert shown in Figure 3.8-(a), the basic dimensions can be obtained by simple trigonometric considerations as

$$
\begin{gathered}
L_{\text {apex }}=\frac{r_{i C}}{\sin (\varepsilon / 2)} \\
L_{o}=\frac{r_{\varepsilon}}{\sin (\varepsilon / 2)} \\
S=\frac{L_{\text {apex }}}{\cos (\varepsilon / 2)} \\
L_{\text {tan }}=\frac{r_{\varepsilon}}{\tan (\varepsilon / 2)}
\end{gathered}
$$




$$
\begin{gathered}
L_{\text {arc }}=r_{\varepsilon}\left\{\left(\frac{\pi}{2}-\psi_{r}\right)\right\}, \quad \psi_{\mathrm{r}} \text { in radians } \\
L_{\text {line }}=S-L_{\text {tan }}
\end{gathered}
$$

The spatial position of the tool corner co-ordinate system can then be expressed relative to the insert co-ordinate system as

$$
X_{l}=0 ; \quad Y_{l}=L_{\text {apex }}-L_{o}
$$

Each of the segments that form the cutting edge are defined by a pair of end-points with coordinates $\left(X_{i} Y_{i}\right)$; logically, $n$ segments would result in $n+1$ points. All points are numbered starting from the extreme tip of the insert corner radius as shown in Figure 3.8(a). A minimum of two segments are needed to define the two distinct regions of the cutting edge, the straight line and the arc regions. A higher number of segments obviously results in a smoother approximation of the edge geometry and is a practical necessity to make a tool designed with this model. The generalized model implementation uses the following rules to assign segments to either the line or arc regions:

For an even number of segments, the segments are evenly distributed in either region. One common point is set at the transition between the line-arc regions. The general notation for the number of points in each region is expressed as

$$
N_{\text {line }}=N_{\text {arc }}=\frac{n}{2}+1
$$

where $n$ is the number of predefined segments and $N_{\text {line, }}, N_{\text {arc }}$ are number of points in the line or arc regions. 
For an odd number of segments, the arc region is assigned one segment more than the straight-line region with one common point assigned to the transition between line-arc regions. The number of points in each region is,

$$
N_{\text {arc }}=N_{\text {line }}=n_{*}+1
$$

where $n_{*}$ is the number of segments assigned to each region and is given by,

$$
n_{\text {arc }}=\frac{n+1}{2} \quad \text { and } \quad n_{\text {line }}=\frac{n-1}{2}
$$

The $\left(X_{i} Y_{i}\right)$ pair for the $1^{\text {st }}$ point at the tip of the tool is fixed and its values are,

$$
X_{1}=0 ; \quad Y_{1}=Y_{l}+r_{\varepsilon}
$$

Based on Figure 3.9, generalized equations can be expressed to define each $\left(X_{i} Y_{i}\right)$ pair along the cutting edge.

Case I: $i \leq N_{\text {arc }} \quad$ (arc segment)

$$
\begin{gathered}
X_{i}=X_{l}+\Delta X_{i} \\
Y_{i}=Y_{l}+\Delta Y_{i}
\end{gathered}
$$

where $\Delta X_{i}$ and $\Delta Y_{i}$ are the incremental values of the $\left(X_{i}, Y_{i}\right)$ pair and are expressed as

$$
\begin{aligned}
& \Delta X_{i}=r_{\epsilon} \sin \left[(i-1)\left\{\frac{(\pi / 2)-(\epsilon / 2)}{N_{a r c}-1}\right\}\right] \\
& \Delta Y_{i}=r_{\epsilon} \cos \left[(i-1)\left\{\frac{(\pi / 2)-(\epsilon / 2)}{N_{a r c}-1}\right\}\right]
\end{aligned}
$$




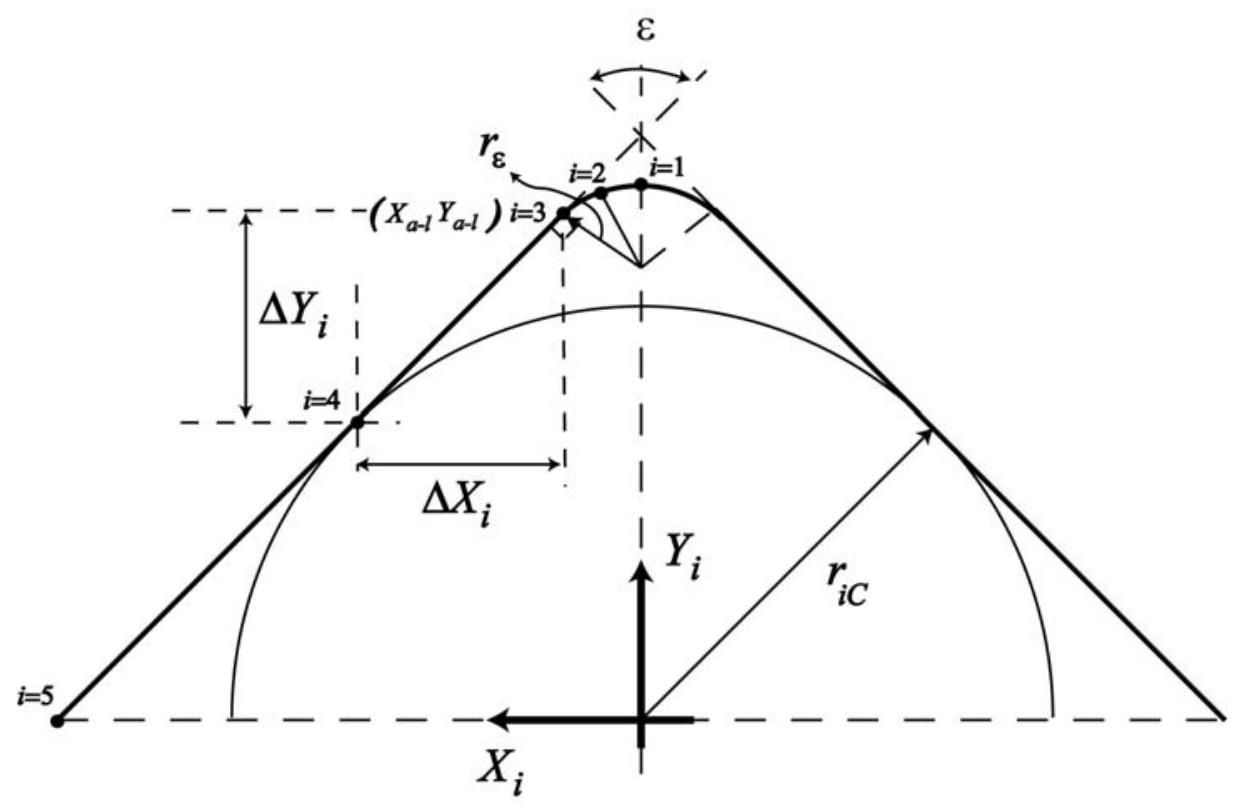

Figure 3.9: Incremental distances of edge segments.

Case II: $i>N_{\text {arc }} \quad$ (line segment)

$$
\begin{gathered}
X_{i}=X_{a-l}+\Delta X_{i} \\
Y_{i}=Y_{a-l}+\Delta Y_{i}
\end{gathered}
$$

where $\left(X_{a-l} Y_{a-l}\right)$ is the coordinate of the common point established at arc-line transition. The incremental values $\left(\Delta X_{i}, \Delta Y_{i}\right)$ for successive points are given by

$$
\begin{aligned}
& \Delta X_{i}=\left(\frac{L_{\text {line }}}{N_{\text {line }}-1}\right)\left(i-N_{\text {arc }}\right) \sin \left(\frac{\varepsilon}{2}\right) \\
& \Delta Y_{i}=\left(\frac{L_{\text {line }}}{N_{\text {line }}-1}\right)\left(i-N_{\text {arc }}\right) \cos \left(\frac{\varepsilon}{2}\right)
\end{aligned}
$$




\subsection{Defining the Inclination Angle of the Cutting Edge}

Equations (3.1) - (3.10) provide only the shape definition for the insert geometry. Designing an elemental inclination angle to guide the chip towards a target point involves two other local variables that need to be defined - the equivalent lead angle $\overline{\psi_{\mathrm{i}}}$ and the exact spatial position of the target within the insert co-ordinate system. In the straightline region, the equivalent lead angle is the insert lead angle $\left(\overline{\psi_{\mathrm{i}}}=\psi_{i}=\varepsilon / 2\right)$. However, the equivalent lead angle continuously varies along the arc region of the insert. The equivalent lead angle at any point in the arc region can be obtained by drawing the line tangent at that point as shown in Figure 3.10 and can be expressed as

$$
\overline{\psi_{\mathrm{i}}}=\left(\frac{\pi}{2}\right)-\left[(i-1)\left\{\frac{\left(\frac{\pi}{2}\right)-\left(\frac{\epsilon}{2}\right)}{N_{\text {arc }}-1}\right\}\right], \quad \overline{\psi_{\mathrm{i}}}, \epsilon \text { in radians }
$$

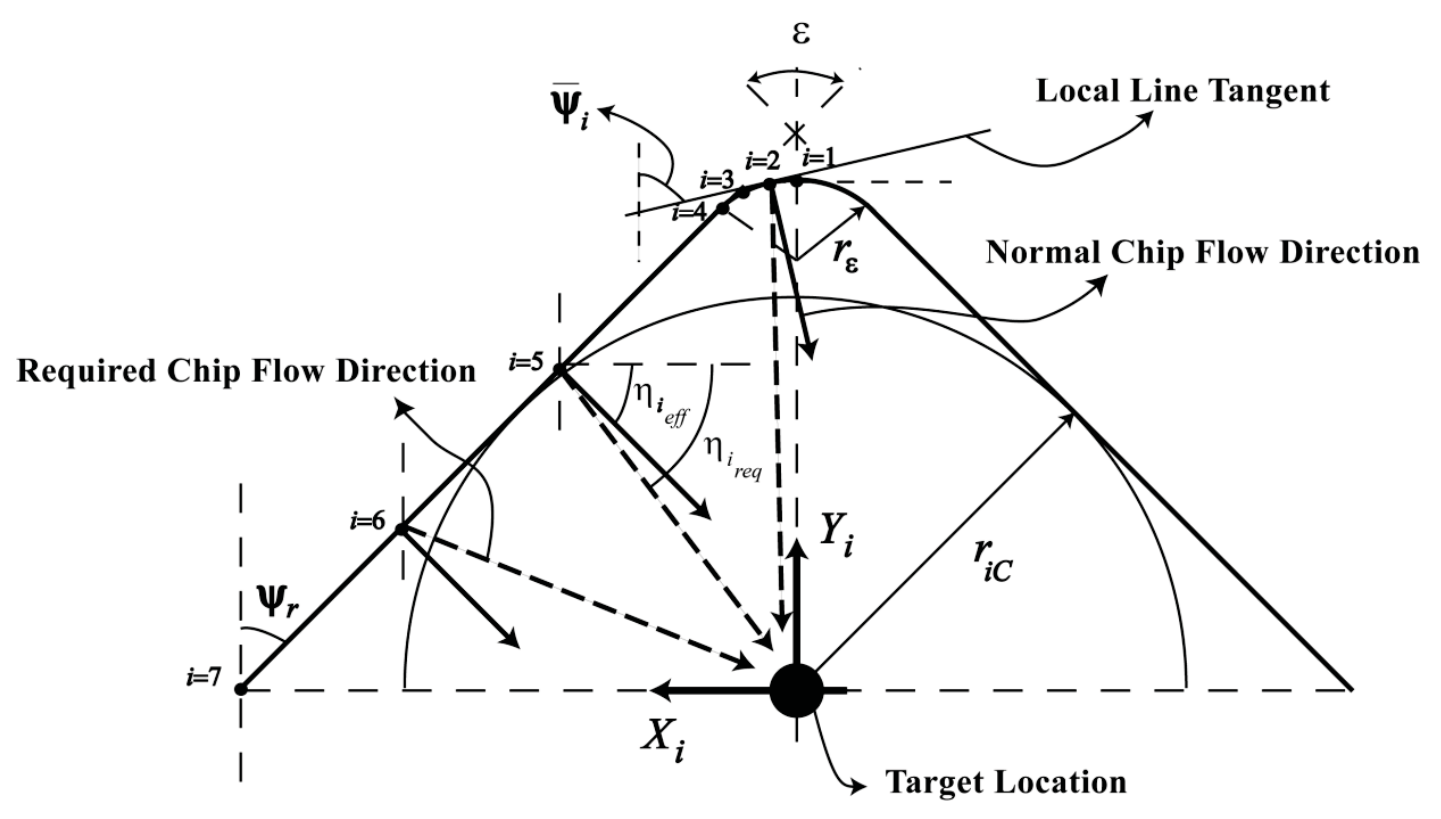

Figure 3.10: Schema for elemental inclinations. 
It can be recalled that within each element, the chip side-flow angle, $\eta_{i_{e f f}}$, is assumed to be in the direction of the normal vector to the line tangent defining equivalent lead angle. From geometric considerations,

$$
\eta_{i_{e f f}}=\bar{\psi}_{\mathrm{i}}
$$

Given that the explicit objective of the model is to direct the chip towards the target, the final task is to design an elemental inclination angle that "redirects" the chip sideflow vector to the intended target. The preferred location for the target is the origin of the insert co-ordinates $\left(X_{i}=0, Y_{i}=0\right)$. Note that the target location may also be any arbitrary spatial location within the insert coordinate system. However, as explained previously, setting the target to the origin of the insert coordinate system is practical and reduces the complexity of the tool system design. Thus, the added inclination required at each segmental endpoint to redirect the chip from its "normal" flow path is simply the difference between the required side-flow angle, $\eta_{i_{r e q}}$, and the effective flow angle, $\eta_{i_{e f f}}$.

$$
\lambda_{i}=\eta_{i_{r e q}}-\eta_{i_{e f f}}
$$

With the available elemental inclination angles, the problem now culminates to defining $Z_{i}$ values in the insert co-ordinate system for individual points along the edge. The cumulative edge inclination is obtained by numerical integration of elemental inclinations which is given by

$$
Z_{i}=Z_{i-1}+\Delta l_{n} \tan \lambda_{i}
$$

where $\Delta l_{n}$ is the length of individual segments obtained by (overleaf) 


$$
\begin{array}{ll}
\Delta l_{n}=\frac{L_{\text {line }}}{N_{\text {line }}-1} & (\text { for line segment }) \\
\Delta l_{n}=\frac{L_{\text {arc }}}{N_{\text {arc }}-1} \quad(\text { for arc segment })
\end{array}
$$

The segmental inclination is shown in Figure 3.11. As an example, a plot of the $\left(X_{i} Y_{i}\right.$ $Z_{i}$ ) coordinates for a S433 insert configuration is shown in Figure 3.12.

To enable a better understanding, two points need to be noted in Figure 3.12:

1) The $Z_{i}$ output is plotted by cutting and unwrapping the edge so that the corner radius appears as a linear plot. This is done to aid visualization of the inclination along the curved tool corner region.

2) The area shown between the thick line representing tool cutting edge and the dotted line represents the reinforcement provided to strengthen the edge. Even though this is labeled as a "land", it is not to be confused with the standard chamfer land provided on cutting tools. The land in the UCB, which will be called reinforcement hereon, in

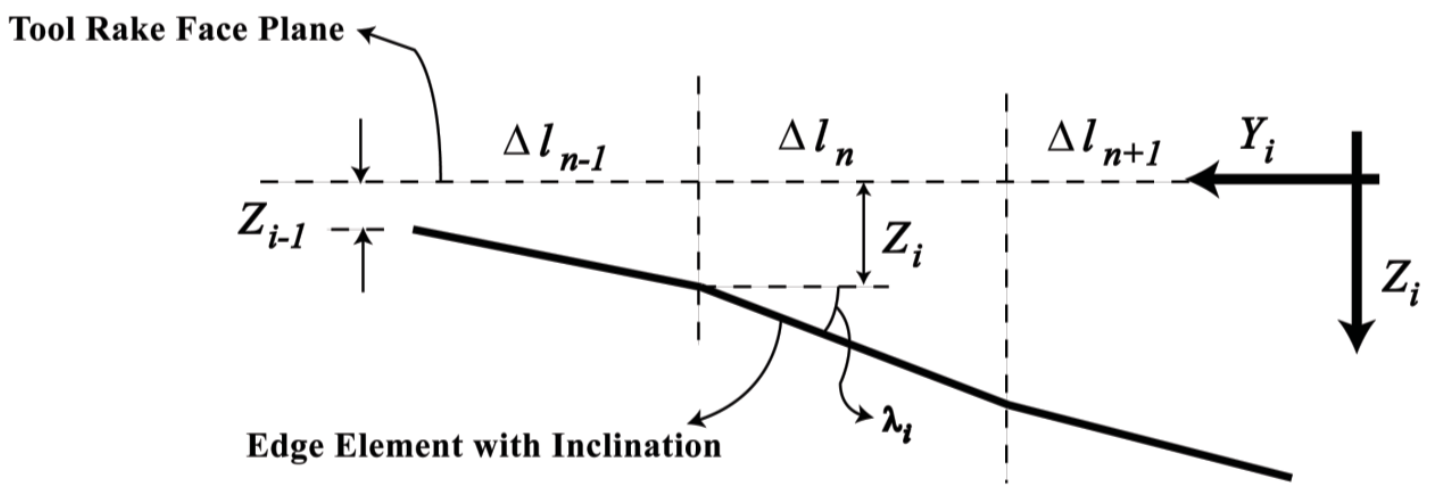

Figure 3.11: Plot of elemental inclination along tool $\mathrm{Y}-\mathrm{Z}$ axis. 


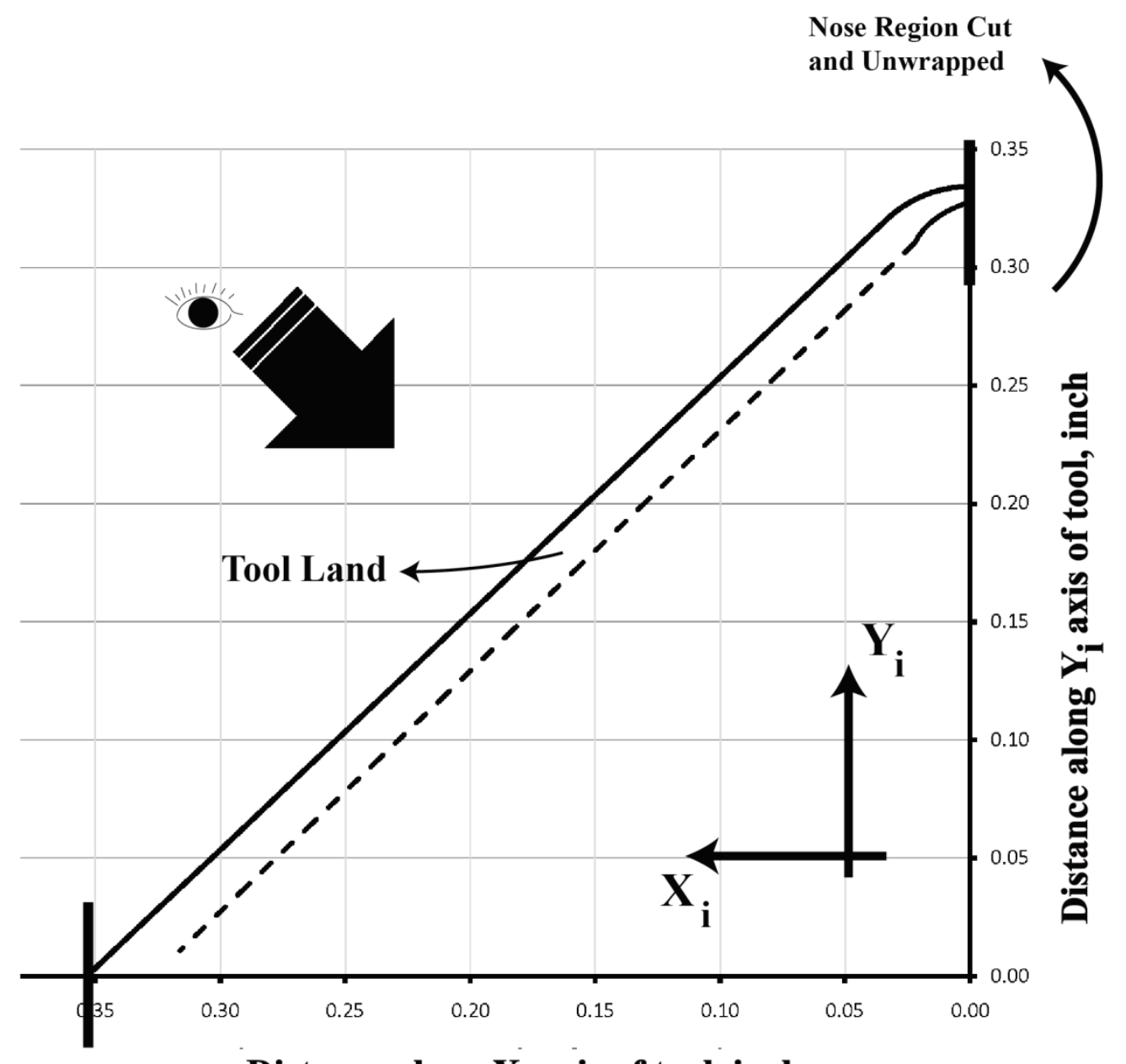

Distance along $X_{i}$ axis of tool, inch

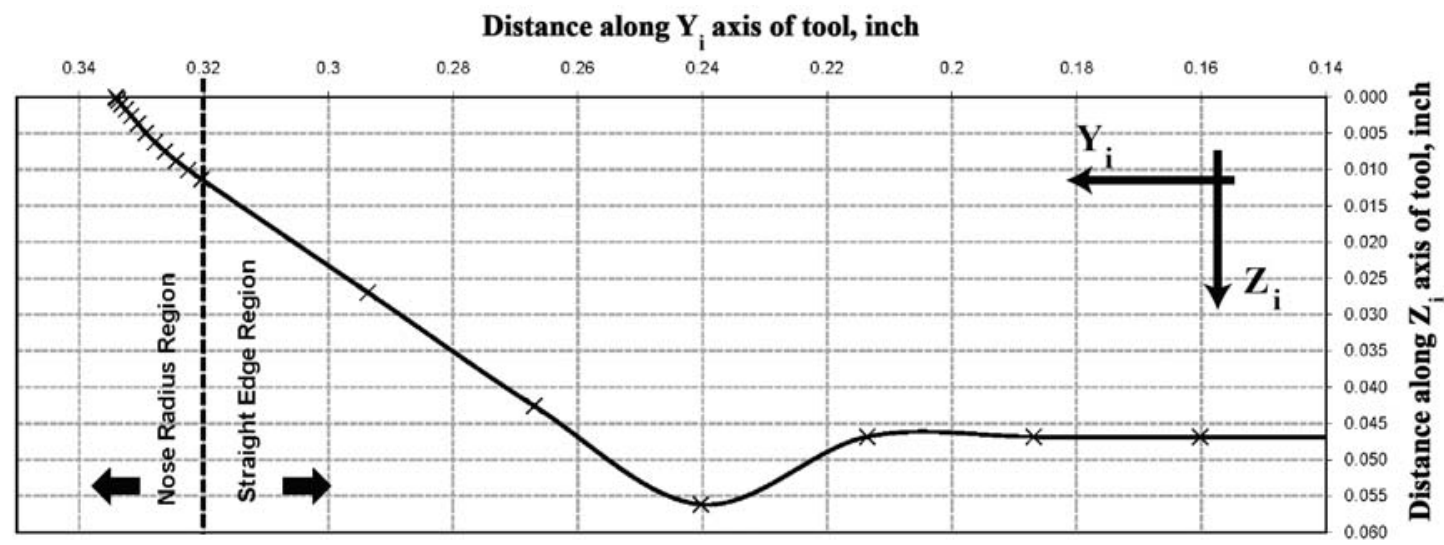

Figure 3.12: Insert edge geometry predicted by the model. 
this work is obtained by transforming the edge coordinates to provide a normal rake angle of $+5^{0}$ in the vicinity of the cutting edge. The width of the reinforcement is included as a user defined feature in the model with the value input as a percentage of the recommended maximum feed (values based on recommendations in Sandvik, 2005). This is explained in the subsequent section on prototype development.

It can be seen from Figure 3.12 that the value of the inclination angle increases moving away from the corner radius region. This is to be expected since the natural tendency of the chip at the tool corner region is to flow towards the target location $\left(X_{i}=0, Y_{i}=0\right.$ in Figure 3.10) situated at the origin of the insert coordinate system. Along the straight edge however, the chip tends to flow normal to the edge requiring a sharp inclination angle to redirect the flow towards this target location.

\subsection{Development of a Tool Prototype}

From the developed model, the $\left(X_{i} Y_{i} Z_{i}\right)$ coordinates are blended with surfaces to realize the actual tool geometry in 3-dimensional Cartesian space. As a simple solution, the proposed model was implemented using a spreadsheet and the output coordinate points exported into ProEngineer CAD to create a model for which the CNC code is generated with its Complete Machining software package. Rather than use specific mathematical techniques to blend the surfaces, ProE's default blending ability is used and the resulting rendering of the tool geometry is shown in Figure 3.13. The final tool rake face geometry resembles the restricted contact tool geometries used by past researchers such as Klopstock (1925), Worthington (1975) and Jawahir (1988) with the difference 


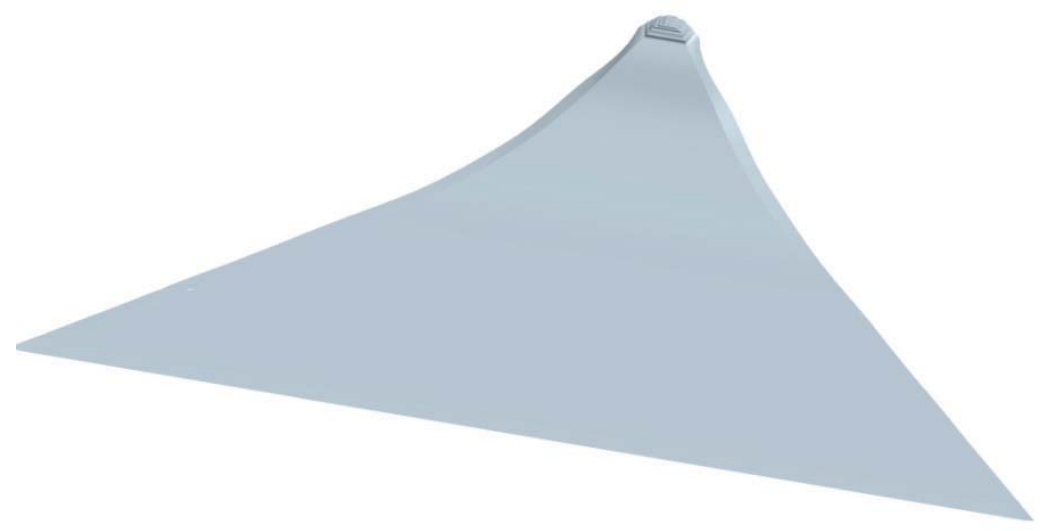

Figure 3.13: Rendering of a $\mathrm{S} 433$ type prototype tool based on model generated edge geometry.

being that the edge inclination angle is continuously varied with edge position in the present case.

In practical tool design, the cutting edge strength is reinforced with a small chamfer, also called the tool land or T-land (SME, 1996). The general recommendation for T-land widths are $70 \%$ of operating feed $(\max )$ value with an effective operating value of $<30 \%$ feed (Stevenson and Agapiou, 2006). Since the model does not include T-land, a reinforcement width of 0.01 " with a normal rake of $+5^{0}$ is provided at the straight edge region to strengthen the cutting edge. This translates to $50 \%$ of the feed for the most commonly recommended value (Sandvik, 2005) for rough/medium machining $(f=0.02 \mathrm{ipr})$ with a 0.5 " $i C$ insert. Further, the reinforcement width is varied along the cutting edge with a value of $0.005 "$ ( $25 \%$ of feed value) at the corner radius region. The reason for a varying width is to mimic the reduced uncut chip thickness at the corner radius region. The normal rake of $+5^{0}$ is provided to the reinforcement to reduce cutting forces at the edge and assist chip flow without any streaming. 
Surfaces generated from the CAD model are processed using Computer Aided Manufacturing (CAM) methods resulting in a $\mathrm{CNC}$ program. A general purpose 3-axis $\mathrm{CNC}$ milling machine (Clausing Kondia FV300-CNC) is used to machine the top form geometry. A standard ball end mill (GARR 545BA) with $\phi=0.125$ " capable of machining hardened material was used to finish machine the top form geometry. All of the prototype tools used in testing use a basic positive insert configuration with a clearance angle of $7^{0}$. The final developed prototype tool geometry is shown in Figure 3.14. Tool geometry characterization done by means of measurement of tool images (see APPENDIX B) indicate that the corner radius as well as the insert size is less than the design specified values. This was due to material removal on the rake face as well as the flank clearance

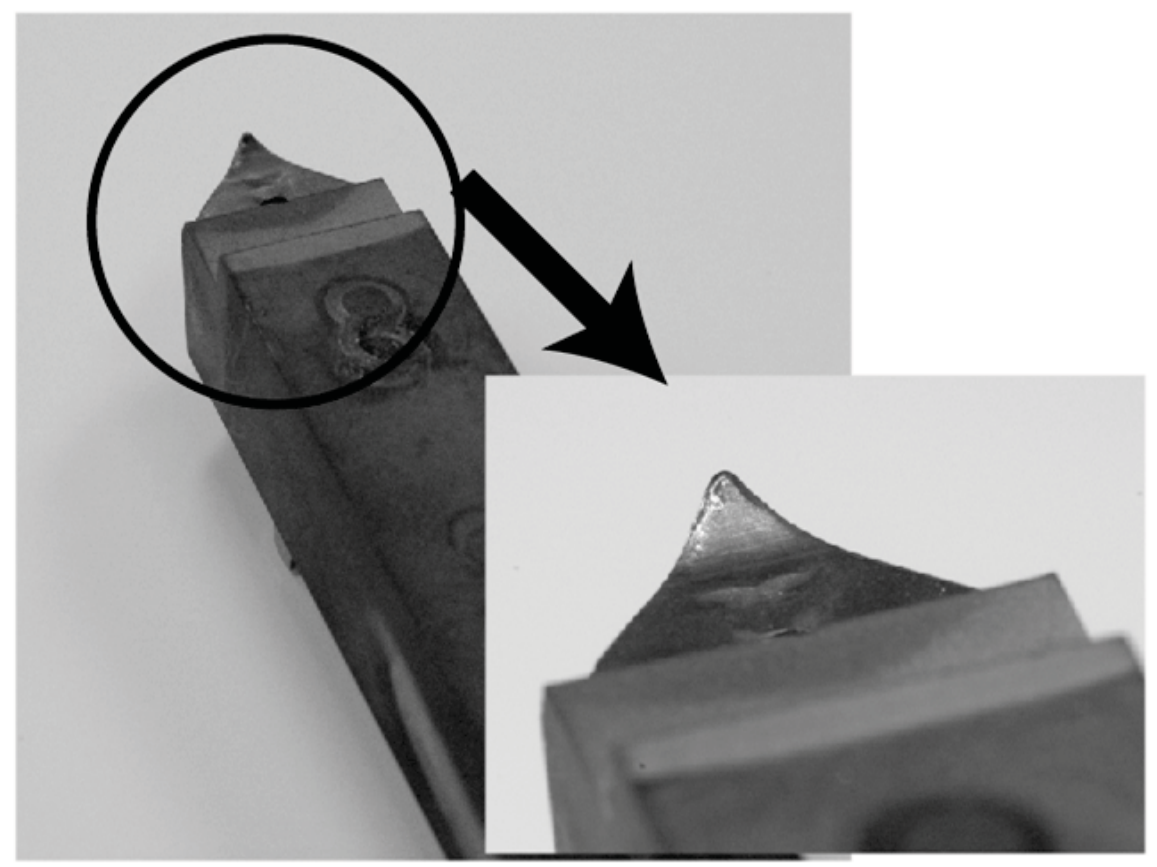

Figure 3.14: Machined prototype of a S433 type tool geometry. 
angles. No action was taken to rectify the lower values. It is noted that the eventual design consideration will need to account for the reduced geometry values by providing extra material stock prior to machining the tool blanks.

\subsection{Concluding Remarks}

While many a research in the past has studied the chip flow as a result of a particular tool geometry, very few work has focused on proactively controlling the chip flow. In this chapter, a model is proposed to control the flow of the chip to reach a pre-designated target point. The fundamental research hypothesis is that "chip flow can be controlled to reach a pre-designated target point by controlling the edge inclination". If true, the extension is to study the feasibility of enabling chip breakage by backward bending. The modeling work addresses only the first part of the problem - that of directing the chip flow to the designated target. The model is based on established theories of chip flow found in the literature. To develop the model, the cutting edge is discretized into a series

of elements. It is assumed that the net chip flow can be directed to a specific target by individually controlling the chip flow from each segment to reach the target location. Based on the model output, a tool prototype is developed and characterized. 


\section{CHAPTER 4}

\section{EXPERIMENTAL STUDIES OF CHIP FLOW WITH A VARIABLE INCLINATION ANGLE}

Results from experimental evaluations of the proposed universal chip control geometry are presented in this chapter. The model-based tool prototype described in Chapter 3 was tested and the results compared with standard commercial insert geometries as a benchmark. Both qualitative and quantitative methods are employed to study the chip behavior during the cutting process.

\subsection{Introduction}

Apart from mechanistic considerations, classical chip control studies also include the use of the visual medium to characterize the chip behavior during machining. Experimental studies of both the flow and breakability of chips are based largely on photographic evidence and subsequent image analysis. The breakability factor is assessed using a post facto analysis of chips collected from the process. On the other hand, the chip flow issue has been treated using in-situ photographs of chip flow during the machining process. Russell and Brown (1966) provide a good overview on measuring the chip side-flow angle using the photographic technique. In general, photographs of 
chip flow during the cutting process are obtained by setting up a camera directly above the tool such that the lens axis is perpendicular to the tool reference plane. From the photographs obtained, chip flow measurements, either the side flow angle or the curl, can then be made offline. In the orthogonal machining setup, the photography technique is convenient since the images are captured directly in the actual plane of chip flow. However, the standard camera setup can be a limitation when used in oblique cutting. Since the camera lens axis is perpendicular to the tool reference plane, the chip flow measurements obtained from image analysis are also in the tool reference plane. Since the actual plane of chip flow in oblique machining is inclined to the tool reference plane, the image perceived by the camera is not a direct measure of the chip side flow. Consequently, the camera needs to be tilted to match the inclination angle of the tool. The other approach to has been to simply transform the chip flow angle to the tool reference plane using the angle of inclination, $\lambda$ (Shaw et al., 1952, Brown and Armarego, 1964). As will be discussed later, the camera tilt solution is not feasible due to the unique geometry of the UCB tool. As such, the orthogonal cutting configuration is used as the basis to investigate chip behavior in the present research.

\subsection{Experimental Details}

Chip kinematics for the model based top form geometry as described in Chapter 3 are investigated via planned bar turning experiments. Figure 4.1 shows the experimental setup used. 


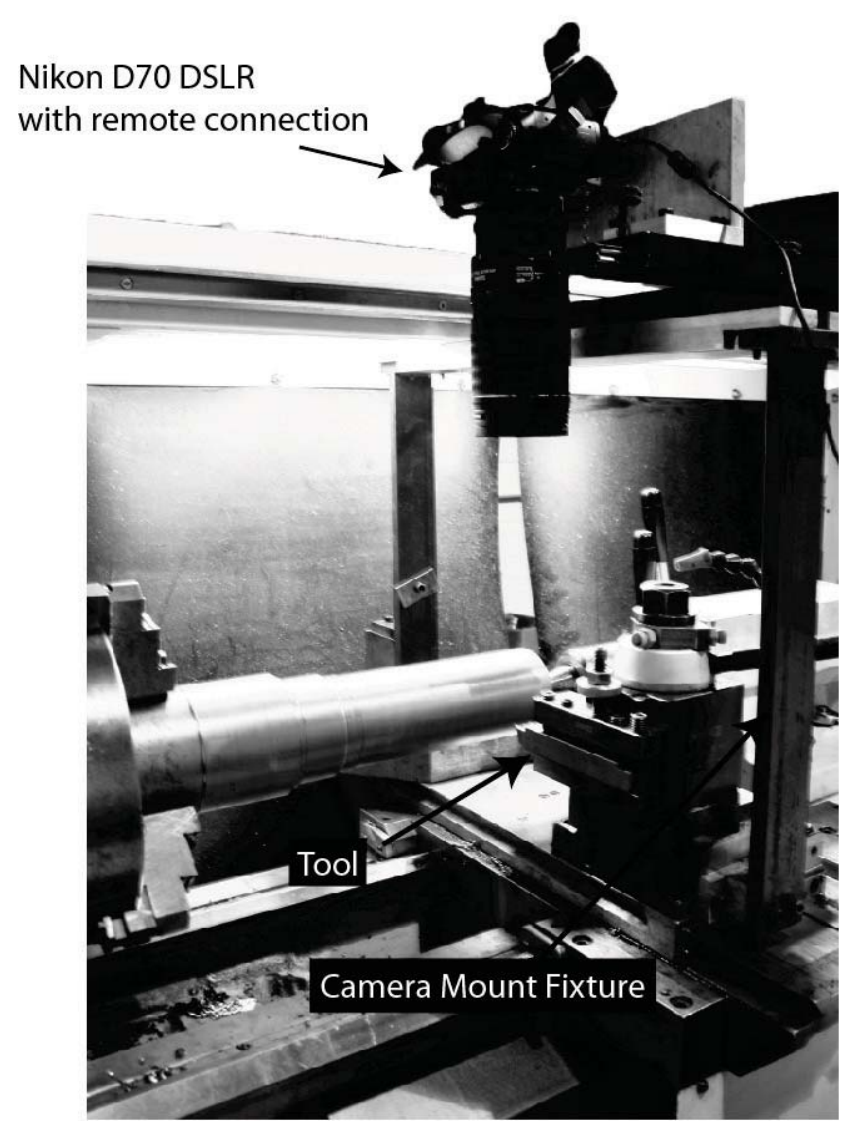

Figure 4.1: Experimental setup for chip flow tests.

All of the experiments were carried out using a standard Colchester Multiturn 2000 $\mathrm{CNC}$ lathe equipped with a $10 \mathrm{HP}$ spindle motor. The cutting tests were run dry with no coolant used. Similar to the method detailed by Russell and Brown (1966), photographs of chip flow during the cutting tests are obtained using a Nikon D70 DSLR camera fitted with a SIGMA $180 \mathrm{~mm}$ macro zoom lens. The standard in-camera flash module provided more than adequate lighting for the experiments without having to resort to an elaborate external lighting setup. A special purpose fixture bolted directly to the lathe carriage was designed to mount the camera during the experiments. Two linear slides operating paral- 
lel to the $\mathrm{X}$ and $\mathrm{Z}$ - axis directions of the machine are provided on the camera mount to enable adequate focal coverage of the area above the tool. No provision is made for vertical height adjustment of the camera since the macro lens provides enough depth of view within its focal range. The camera is operated remotely via a PC and controlled using Nikon's proprietary Camera Capture 4 software. With the shutter speed fixed at the maximum possible value (limited to $1 / 500 \mathrm{sec}$ for remote camera control), only the aperture is varied to ensure adequate depth of field focus. A series of continuous photographs are captured using the high speed burst mode. Even though the camera specification lists high speed photography at 4.5 frames/sec, the remote camera control setup ensures only about 1.5 frames/sec (approximate as measured) due to data transfer limitations. Note that the term "high speed photography" used here is relative and does not imply the standard definition of $>128$ frames/sec used in the photographic domain. While a true high speed system would be the ideal wish list (refer cited works of Jawahir's group to grasp this significance), the exorbitant cost of such a system weighs in on the eventual choice. For this research, the present rate of 1.5 frames/sec served as adequate means to record the requisite information on chip flow behavior.

Two different work materials were used in the experiments - AISI 1018 steel and the aluminum alloy AL 2024. Both are long chipping materials and considered particularly challenging for chip control. Material properties as listed by the supplier (Speedy Metals, 2010) are shown in Table 4.1. Measured hardness values tended to be slightly lower than that listed. Both the work materials used were approximately 4" diameter and 24" long at the outset. 
Table 4.1: Experimental work material properties.

\begin{tabular}{|c|c|c|c|c|}
\hline \multirow{2}{*}{ Material } & \multirow{2}{*}{\multicolumn{2}{|c|}{ Composition (Wt. \%) }} & \multicolumn{2}{|c|}{ Hardness } \\
\hline & & & Listed & Measured \\
\hline $\begin{array}{l}\text { AISI } 1018 \\
\text { medium carbon } \\
\text { steel (cold rolled) }\end{array}$ & $\begin{array}{l}\text { Carbon }(\mathrm{C}) \\
\text { Manganese (Mn) } \\
\text { Phosphorus (P) } \\
\text { Sulfur (S) }\end{array}$ & $\begin{array}{l}0.15-0.2 \\
0.6-0.9 \max \\
0.04 \max \\
0.05\end{array}$ & $\begin{array}{c}\sim 167 \\
\mathrm{BHN}\end{array}$ & $\begin{array}{c}\text { 80-84 HRB } \\
\text { (converts } \\
\text { to } \\
\sim 155 \mathrm{BHN} \text { ) }\end{array}$ \\
\hline AL 2024 & $\begin{array}{l}\text { Manganese }(\mathrm{Mn}) \\
\text { Silicon }(\mathrm{Si}) \\
\text { Chromium }(\mathrm{Cr}) \\
\text { Copper }(\mathrm{Cu}) \\
\text { Iron }(\mathrm{Fe}) \\
\text { Zinc }(\mathrm{Zn}) \\
\text { Magnesium }(\mathrm{Mg}) \\
\text { Other (Oth) } \\
\text { Aluminum (Al) }\end{array}$ & $\begin{array}{l}0.3-0.9 \\
0.5 \\
0.1 \\
3.8-4.9 \\
0.5 \\
0.25 \\
1.2-1.9 \\
0.05-0.15 \\
\text { remaining }\end{array}$ & $\begin{array}{c}\sim 120 \\
\mathrm{BHN}\end{array}$ & $\begin{array}{l}\text { 65-70 HRB } \\
\text { (converts } \\
\text { to } \sim 105 \\
\text { BHN) }\end{array}$ \\
\hline
\end{tabular}

*Source: www.speedymetals.com

Standard square insert geometries were used in the experimental tests. The model based prototype tool (hereinafter termed "UCB" or, Universal $\underline{\text { Chip }}$ Breaker insert) was machined using hardened A-2 tool steel ( 60-62 HRC) as described in Chapter 3. Commercially available inserts with chip breakers recommended exclusively for the respective work materials were used as baseline inserts to characterize the chips. For the AISI 1018 steel, the insert of choice was a Sandvik Coromant SCMT-433-PM, ISO Ptype coated insert recommended for medium/rough cutting conditions. For the AL 2024 work material, a Korloy SCGT-432-AK, ISO K-type uncoated insert (with buffed rake 
face) was selected. The insert choice for aluminum was dictated by the availability of inserts dedicated specifically for machining of aluminum. Not many commercial insert vendors service the aluminum machining market. Among the major vendors, Sandvik, Korloy and Tungaloy carry standard catalog listings (certain geometries only) for such inserts while the others consider this as a special insert. The eventual choice of the Korloy insert was based largely on its ready availability. It was noted that the geometry and design (carbide grade, chip breaker and rake geometry) of the Sandvik and Korloy inserts seem nearly identical. Most inserts available for machining aluminum alloys are supplied uncoated. Also, commercial inserts designed to cut aluminum invariably include a small positive inclination angle, as is the case with the Korloy insert selected. No flat rake face inserts with a chip breaker geometry for cutting aluminum were available. Further, no technical information is available on the exact value of the inclination angle provided on the Korloy insert. Measurements with a tool makers microscope indicated that the inclination was in the range of $8^{0}-10^{0}$, which is an approximate value since no CAD drawings are available for confirmation. Both the insert varieties - Sandvik and Korloy - are of the basic positive cutting geometries and were used with standard Sandvik tool holders ${ }^{1}$ with $\psi_{r}=45^{0}(\mathrm{SSDR} 2525 \mathrm{M})$ and $\psi_{r}=15^{0}(\mathrm{SSBCR} 2525 \mathrm{M})$ configurations for the specific cutting experiments. Figure 4.2 shows the inserts and the respective chip breaker geometries used in the cutting experiments.

In the model, the natural chip side flow was determined using Colwell's model, which, as may be recalled, is applied only to tools with $\lambda=0^{0}$. As a control experiment

\footnotetext{
${ }^{1}$ The assistance of M/s John Frankenstein and Chuck Burhans from Central Industrial Supply as well as Sandvik Coromant in supplying the Sandvik tools free of cost is gratefully acknowledged.
} 

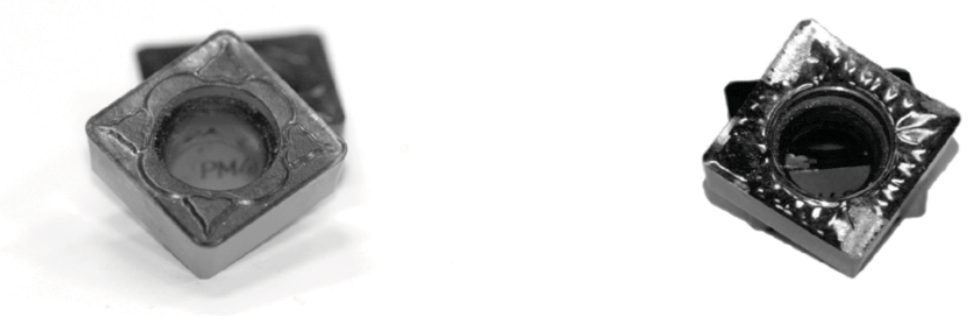

Figure 4.2: Cutting inserts and chip breakers used in the cutting test (left) Sandvik SCGT-433-PM (right) Korloy SCGT-432-AK.

to validate the use of Colwell's model, a flat insert design with no chip breakers and with zero inclination angle was also considered for the cutting tests. This mandates that the flat faced insert be used with the screw-on type of tool holder design (Sandvik, 2009) to ensure that the natural chip flow is unaltered. However, plain flat rake faced screw-on type carbide inserts (without chip breakers; SCMA-433 insert varietal) are not readily available since, in general, flat rake faced carbide insert configurations are commercially available only for a top clamp-on type tool holder design (without screw hole; SCMN433 insert varietal). The use of a top clamp-on type tool holder presents a large obstruction on the rake face and directly in the path of the natural chip flow, thus negating the design of experiments. As such, a flat rake insert geometry was machined with hardened A-2 tool steel $(\sim 60-62 \mathrm{HRC})$ with the rake face ground and polished $\left(\mathrm{R}_{\mathrm{a}}<0.006 \mu \mathrm{inch}\right)$ to have minimal friction effects on chip flow. The flat rake faced insert was used only for the experiments involving AISI 1018 steel. Cutting tests on AL 2024 with a flat rake faced insert was impossible due to heavy build up on the tool edge. 
All of the tool geometries used were adequately characterized - tool corner radii were measured for both the commercial inserts as well as the flat tool. The UCB tool was characterized as described in Chapter 3.

\subsection{Experimental Methodology}

To study the influences of some of the important variables on chip flow, a multi level, multi variable experimental design was developed for the bar turning tests. The complete experimental design is shown in Table 4.2.

The three levels of feed and depths of cut were selected to represent the largest possible band within the recommended values for the commercial inserts. In practice, the feed and depth of cut recommendations depend on the chip area and the type of cutting operation, i.e., rough, medium or finish machining. For any given insert, maximum values for feed rates are based on the insert corner radius while the maximum depths of cut are based on insert size (Sandvik, 2005). In the context of chip breaking, cutting feeds and depths of cut are specified based on the dimensions of the chip breaker geometry (Sandvik, 2009). Thus, the recommended feeds and depths of cut for chip breaking are a subset of the maximum feed/depth values. The recommended cutting parameters for the Sandvik and Korloy inserts are shown in Table 4.3. Note that these values are based on numbers listed on the insert packing box itself rather than from the catalog. Numbers in parenthesis indicates the cutting range over which the recommended chip breakers are supposed to effectively break the chips. 
Table 4.2: Variables used in the experimental design.

\begin{tabular}{ll}
\hline \hline & \multicolumn{1}{c}{$\underline{\text { AISI 1018 }}$} \\
Tools Used & Flat rake faced S-433 \\
& Sandvik SCGT-433-PM $\quad$ (coated grade GC4225) \\
& UCB S-433 \\
Lead Angle & $\Psi_{r}=45^{0} ; \quad \Psi_{r}=15^{0}$ \\
Cutting Parameters & Feed (ipr): $0.005,0.008,0.014$ \\
& $\begin{array}{l}\text { Depth of cut (inches): } 0.025,0.080,0.120 \\
\text { Cutting Speed (sfm): } 110\end{array}$ \\
\hline
\end{tabular}

\section{$\underline{\text { AL } 2024}$}

Tools Used

Korloy SCGT-432-AK (uncoated grade H10)

UCB S-432

Lead Angle

$\Psi_{r}=45^{0} ; \quad \Psi_{r}=15^{0}$

Cutting Parameters

Feed (ipr): $\quad 0.004,0.008,0.012$

Depth of cut (inches): $\quad 0.025,0.050,0.100$

Cutting Speed (sfm): 225

Given that the UCB tooling is fabricated from A-2 tool steel rather than carbide, the cutting speeds chosen are conservative and are based on recommended values for uncoated HSS tools used in turning operations. The Machinery's Handbook (2000) recommends a speed of $110 \mathrm{sfm}$ for AISI 1018 steel (125-175 BHN) and a speed of 250 
Table 4.3: Recommended cutting parameters for baseline inserts*.

\begin{tabular}{cccc}
\hline \hline Insert & $\begin{array}{c}\text { Feed } \\
\text { (inch/rev) }\end{array}$ & $\begin{array}{c}\text { Depth of cut } \\
\text { (inch) }\end{array}$ & $\begin{array}{c}\text { Cutting Speed } \\
(\mathbf{s f m})\end{array}$ \\
\hline Sandvik S433 & 0.011 & 0.038 & 450 \\
& $(0.006-0.017)$ & $(0.028-0.142)$ & $(325-655)$ \\
Korloy S432 & 0.008 & & \\
& $(0.004-0.016)$ & 0.06 & 1145 \\
& & $(0.02-0.24)$ & $(970-1415)$ \\
\hline \hline
\end{tabular}

*Based on values from insert packaging.

sfm for AL 2024 (90-130 BHN). These speeds may very well be increased by using carbide materials or coatings.

Adequate care was exercised to ensure that the experiments were run under controlled conditions. Cutting heights of all tools were set to within $+/-1 / 64$ inch of the lathe axis. The experiments were run dry with the actual cutting time approximately between 8-10 seconds. All experiments were replicated three (3) times. To reduce the wastage of work material, the depth of cut was blocked over one specific level within a replication. Cutting feeds and tools used (Flat, Sandvik or UCB) were randomized within the given replication. The complete run order is furnished in APPENDIX C. Further, since the location of the cut - either at the workpiece center or towards the tailstock or headstock ends - could potentially change the chip morphology, the cutting location itself was randomized over the length of the workpiece. To minimize the depth of cut variations, the workpiece was turned to size before each replication. Recognizing that build up was in- 
evitable due to the dry cutting mode, the rake face immediately adjacent to the cutting edge (on the reinforcement surface) was honed with a fine grit stone prior to each cut. A fresh cutting edge was used for each replication.

\subsection{Methodology for Chip Flow Measurements}

As described previously in Section 4.1, photographs of the chip flow were taken with the camera focused on the tool reference plane. Usually, in oblique machining, the camera lens axis is tilted to be perpendicular to the inclined rake face (Venuvinod and Shing, 1977) so as to obtain images in the actual plane of chip flow. Such an arrangement is straightforward when using a flat rake-faced tool in an oblique setting, but the same cannot be implemented in this experiment since the inclination of the UCB varies along the edge. As a compromise, the photographs are taken with the focus on the tool reference plane.

The photographic images were recorded at a speed of 1.5 frames/second (approx). For the cutting time of 8 seconds, a total of 10-15 images were recorded. From the collection, 3-4 images correlating to the middle of the cutting time were selected. It is assumed that the chip flow attains a quasi steady state in this time interval and, as such, the images are deemed as a good representation of the actual chip behavior. In general, all of the images (across all replications) seemed to be fairly consistent vis-à-vis chip flow; images with a changed chip flow due to external obstruction such as either build up or chip debris were not considered for analysis. One image from each representative selection was used and the chip side flow angle measured using Adobe Illustrator 10. Dimensional measurements within the image were calibrated both horizontally and verti- 
cally using a separate image of graduations on a metal scale (1/64th graduation marks) photographed at the same focal length as the cutting edge (see APPENDIX B). Prior to the measurement, the chip image was "touched up" to eliminate the shadows that were invariably present in the cutting edge region. The image stabilization process ensured that the chip edge was clearly visible for measurement upto the tool cutting edge.

Several shortcomings on photographic measurements of chip side flow have been identified in the past. In reality, straight flow of chips are rare and more often, the common form attained by chips are the $3 \mathrm{D}$ variety. Separating the straight part from the curled part in a chip is almost impossible and it is often discretionary to the observer (Kharkevich and Venuvinod, 1993). It is plausible that one could draw a straight line from the chip root (connection to the workpiece) and subjectively still obtain a range of angles representing the side-flow angle. To address this issue, a curve following the curl of the chip edge is drawn on the chip image and the side flow angle is assumed to be between the tangential line to this curve at the chip root and the feed direction.

Venuvinod and Shing (1977) note yet another unique observation on photographic measurements of chip side-flow angle in oblique cutting. Due to the angle of inclination between the actual flow plane and the "perceived" image in the tool reference plane, the two edges of the chip seem to flow at different angles. Venuvinod and Shing's solution involved measuring the flow angle on both edges and obtaining the mean flow angle. Such a procedure may very well equate to measuring the side flow angle at the center of the chip width. In fact, in a related earlier work, Spaans (1970) based his side flow results on measurements taken in the middle of the chip width. In this research, the methodology used is similar to the one used by Venuvinod and Shing. It was observed 
that the difference of the side flow angle between the two edges was in the range of $2-4$ degrees. In a majority of cases involving the flat faced tool and the commercial tools (Sandvik and Korloy), the tight up-curling of the chip meant that only one edge was available for measurement. Due to the small variation seen between the two edge readings, it was assumed that the single edge measurement adequately defined the chip flow angle, especially at $\lambda=0^{0}$. Figure 4.3 shows the methodology of measurement for chip side flow angle. Note that the tool edge boundary is highlighted for ease of measurement. The boundary is generated from a photograph of the insert before entering the cut.

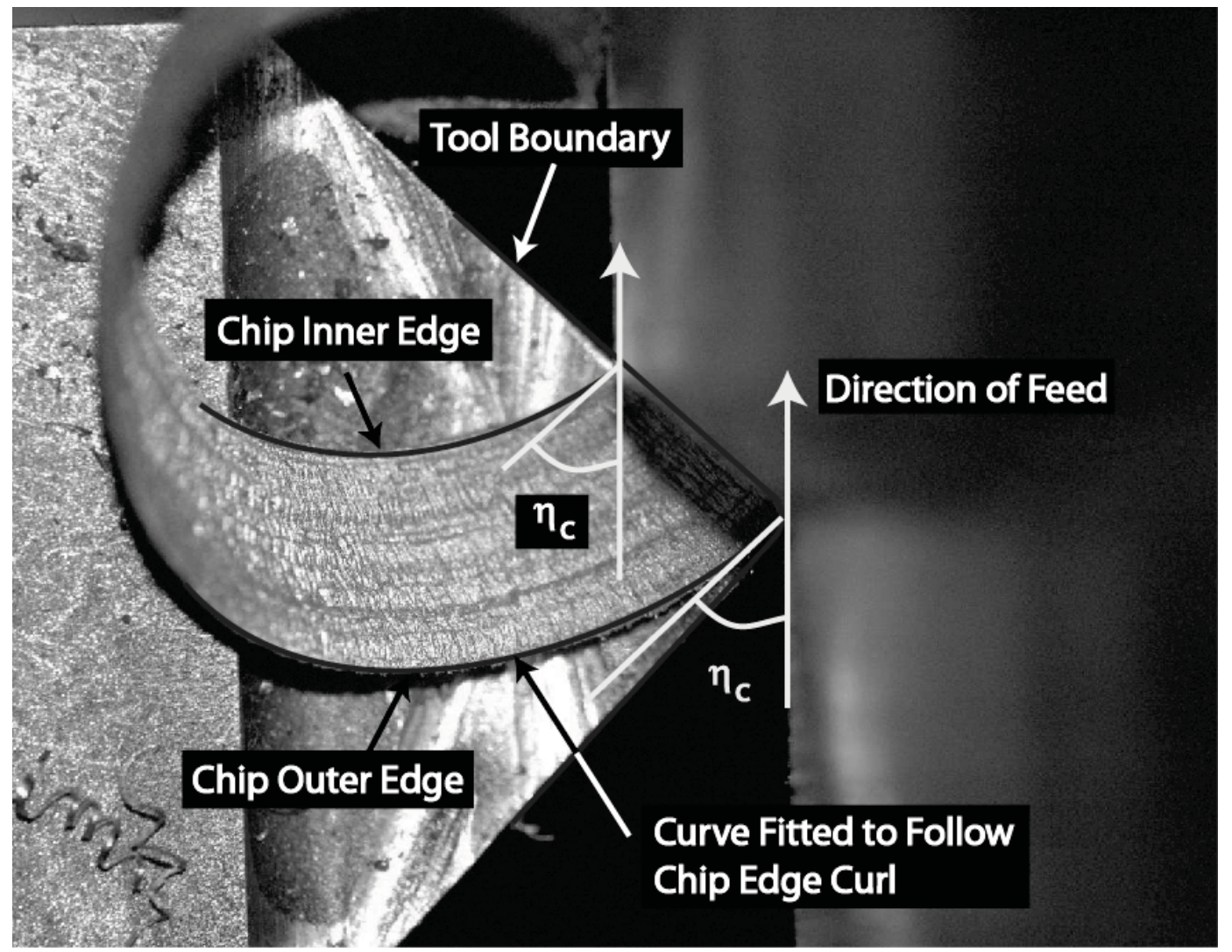

Figure 4.3: Methodology for chip flow measurements. 


\subsection{Results of Experiments}

Results from the photographic measurements along with the standard errors for the two different work materials and lead angles are shown in Figure 4.4-4.7. All results plotted are the averages of replicated experiments (see APPENDIX D for tabulated values). The results are plotted with the chip side flow angle, $\eta_{c}$, on the ordinate and the depth of cut, $d$, on the abscissa for varying levels of feed.

In the case of AISI 1018, in order to avoid over populating any single graph with the large number of datasets available, results for the two baseline tools used, i.e., the flat faced tool and the Sandvik insert, are plotted separately along with the predicted values from Colwell's model for comparison. Plots for the baseline tools at $\psi_{r}=45^{\circ}$ and $\psi_{r}=$ $15^{0}$ are shown in the left-hand column of Figure 4.4 and Figure 4.5 respectively. Plots in the right-hand column of Figures 4.4-4.5 show measured side flow angles from the UCB cutting tests. Also shown in the latter plots are the values of side flow angles required for the chip to flow to the specified target point $\left(X_{i}=0, Y_{i}=0\right)$. The required side flow angles are calculated from pure geometric considerations. Recall from Chapter 3 that the model used to generate the tool top form geometry provides discrete inclination angles to direct chip flow from each edge element toward the target point. In reality however, the chip flows towards the target as a single entity and hence, the side flow angle for the entire chip can also be measured from the center of the chip width (Spaans, 1970). In this work, the theoretical chip side flow angle is derived using the chip center as reference. The chip center location is calculated using the mid-point formula based on the end coordinates of the line representing the equivalent cutting edge from Colwell's model. A line 


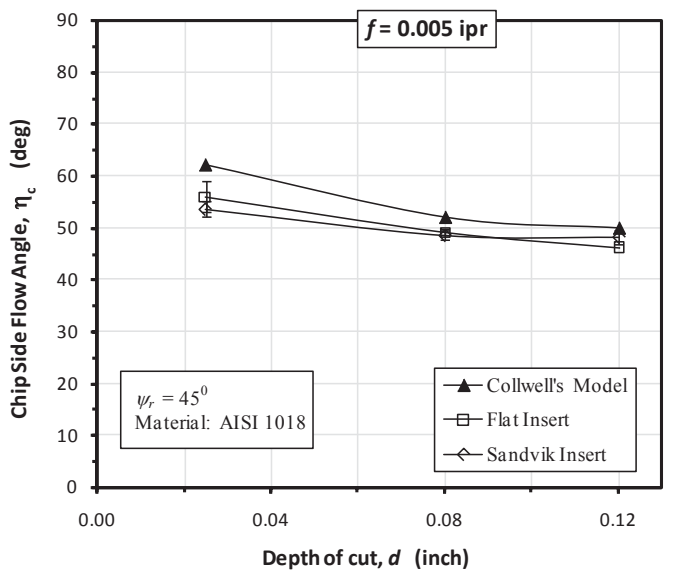

(a)

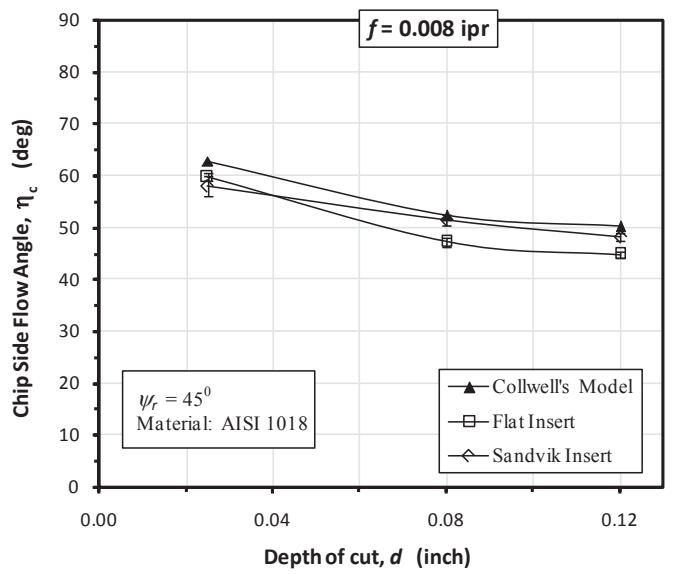

(b)

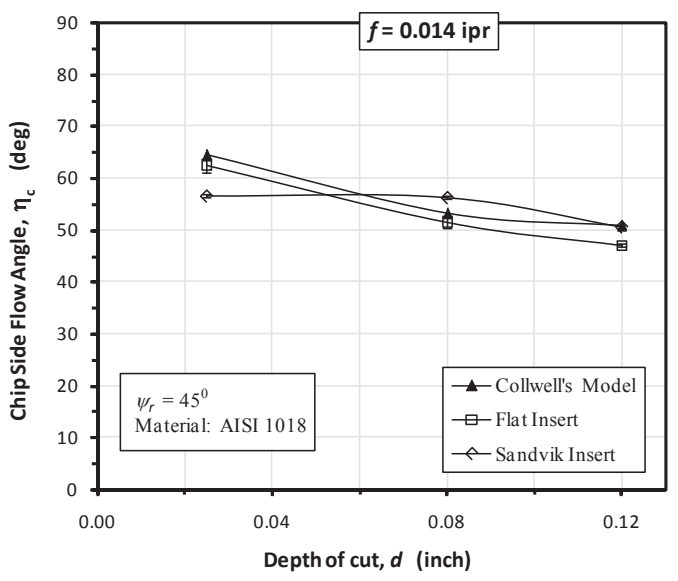

(c)

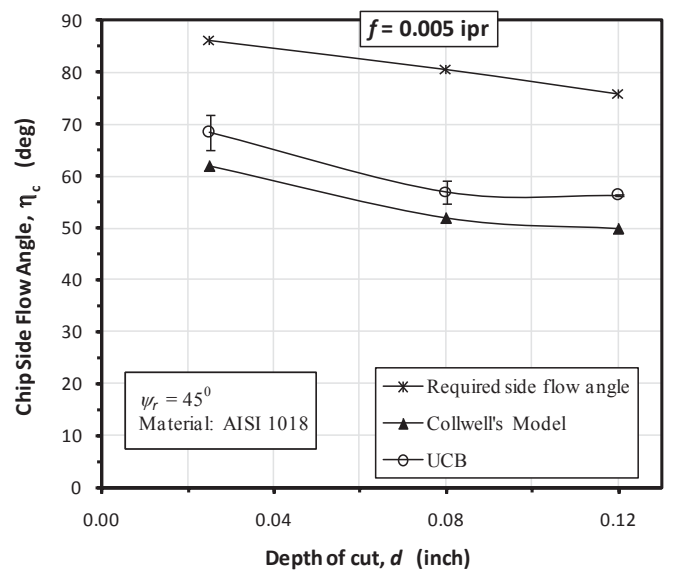

(d)

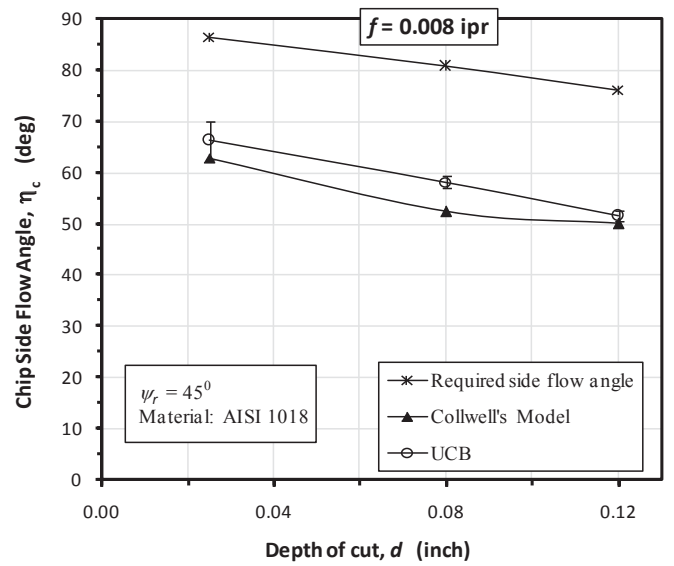

(e)

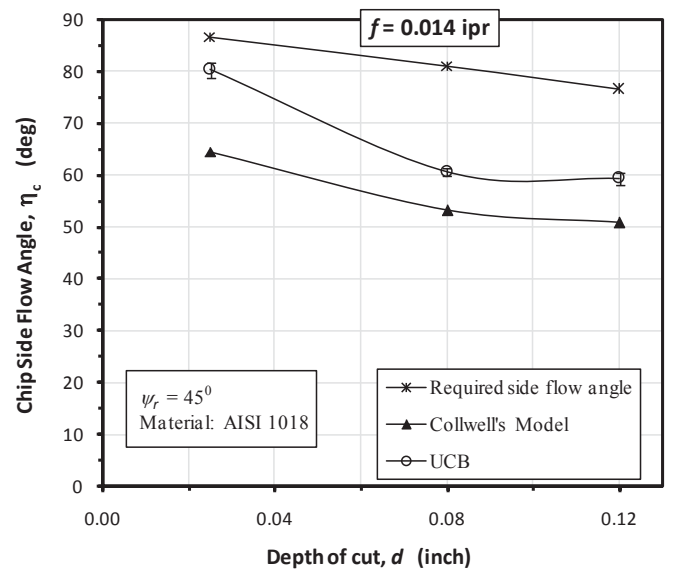

(f)

Figure 4.4: Measured chip side flow angles for: (a)-(c) Baseline tools (d)-(f) UCB tool; Work material: AISI 1018, $\psi_{r}=4^{\circ}$. 


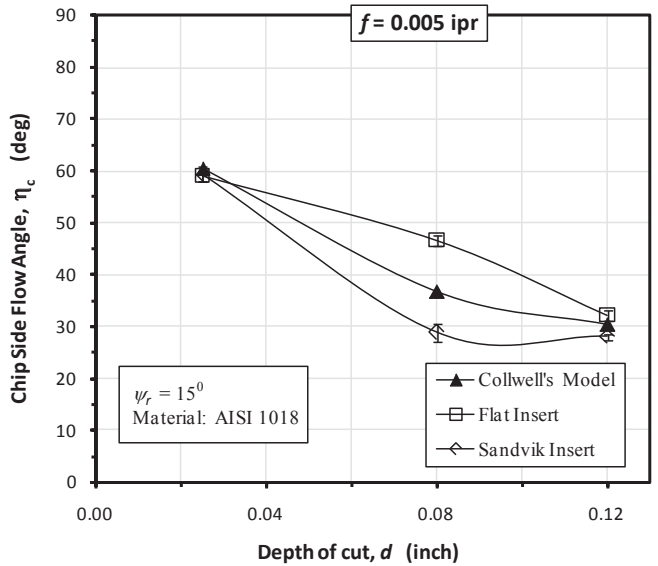

(a)

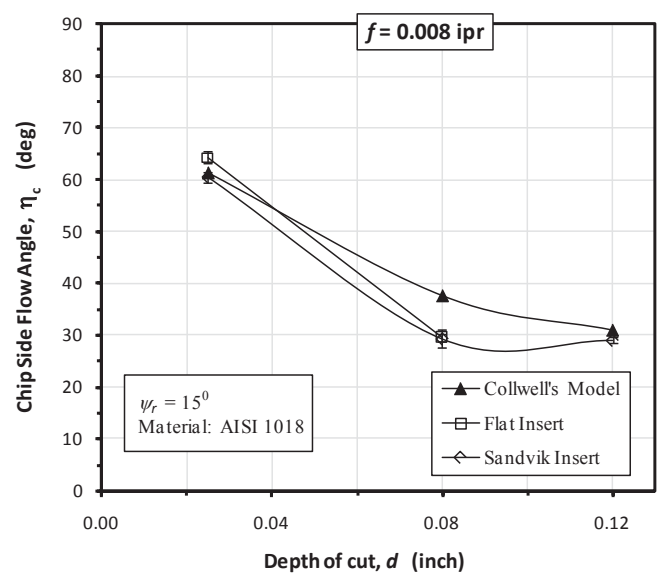

(b)

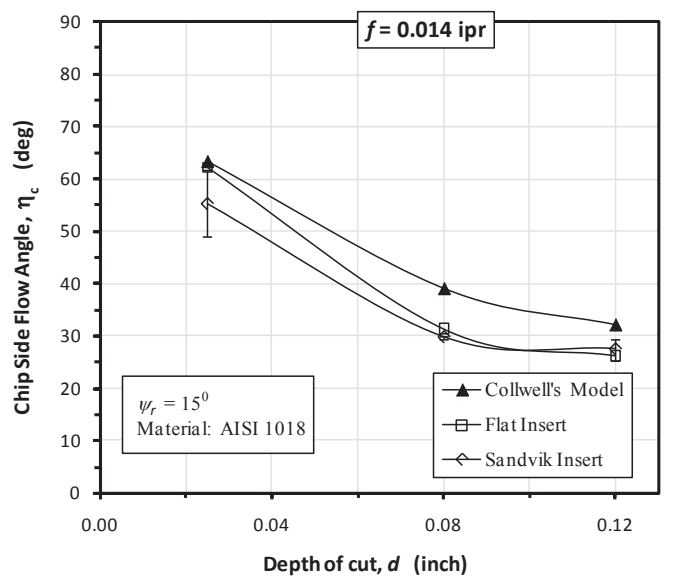

(c)

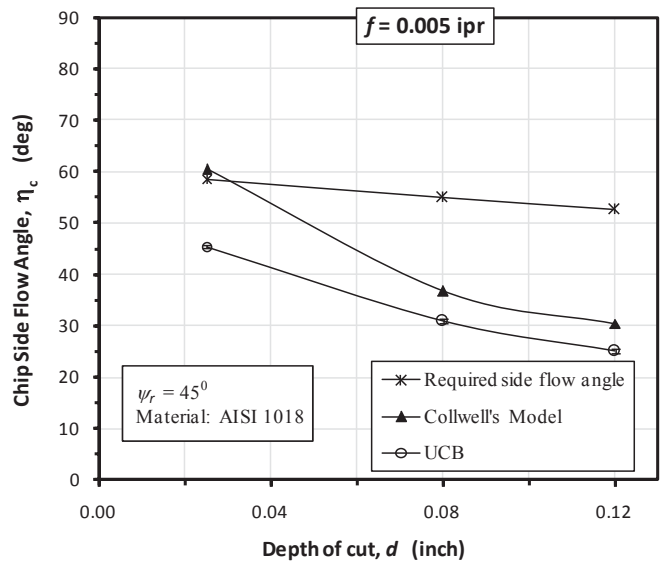

(d)

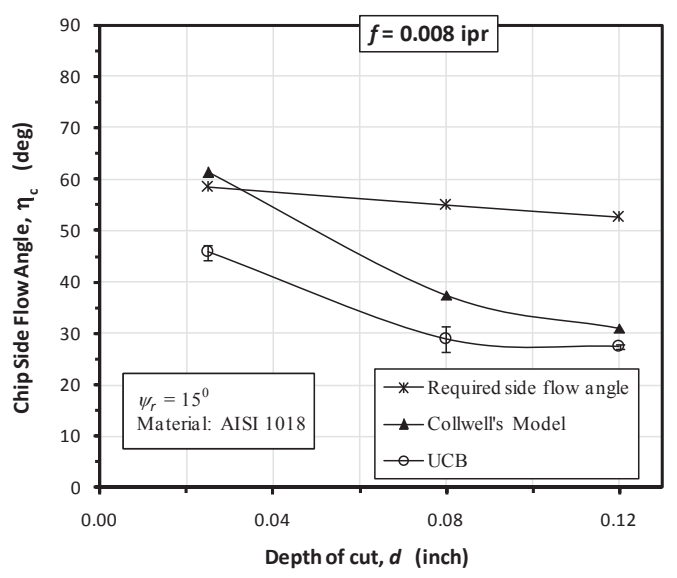

(e)

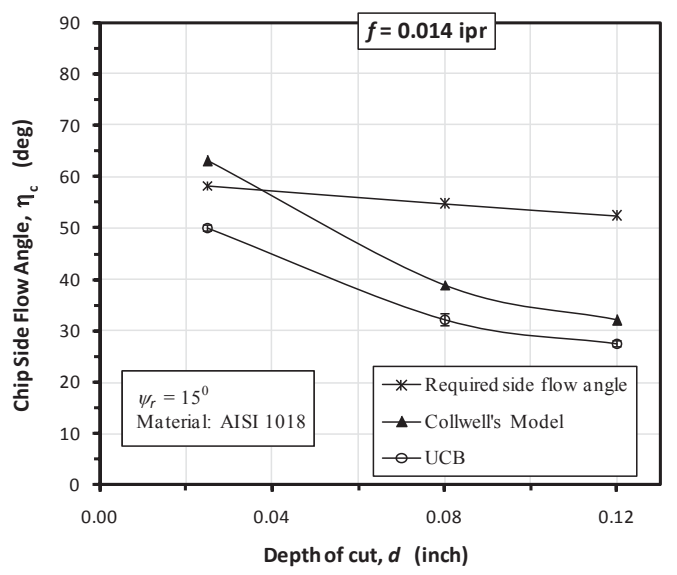

(f)

Figure 4.5: Measured chip side flow angles for: (a)-(c) Baseline tools (d)-(f) UCB tool; Work material: AISI 1018, $\psi_{r}=15^{\circ}$. 
connecting the chip center with the target point indicates the required chip side flow direction and the required chip side flow angle is the angle formed between this line and the direction of feed. Note that the values from Colwell's model are also included as a reference in the UCB plots and provides a simple means to compare the relative values between tests with the baseline tools and those with the UCB tool.

Considering the results for AISI 1018 from Figures 4.4 - 4.5, the following trends are observed:

1. As can be expected, the results trend follows the asymptotic curve seen when cutting with a tool corner radius tool (Young et al., 1987). A higher value of chip side flow is seen at low depths of cut when the cutting process is dominated by the corner radius. At larger depths of cut, the chip side flow angle decreases due to the involvement of the straight cutting edge of the tool.

2. For both $\psi_{r}=45^{\circ}$ and $\psi_{r}=15^{0}$, Colwell's model tends to over-predict the side flow angles when compared to the measured values for the flat rake faced tool as well as the Sandvik insert. Deviations from the general trend is observed at two specific cases, (a) Sandvik, $\psi_{r}=45^{\circ}, d=0.08$ inch, $f=0.014$ ipr and (b) Flat tool, $\psi_{r}=15^{0}, d=$ 0.08 inch, $f=0.005 \mathrm{ipr}$, and were noted to be due to the action of the chip breaker design in the Sandvik insert and build up in the case of the flat tool. No attempts were made to rerun the tests since the variations observed are the result of a common cause within the machining system.

Note the missing data point in the case of the flat rake face tool at $\psi_{r}=15^{0}, d=$ 0.120 inch, $f=0.008 \mathrm{ipr}$, which is due to tool failure. In fact, repetitive tests at the 
large depth/feed case, i.e., $d=0.120 \mathrm{inch}, f=0.014 \mathrm{ipr}$, run before this particular test resulted in edge failure of no less than four (4) consecutive tool edges and hence further testing was abandoned.

3. Based on Figure 4.4, for the UCB cutting tests at $\psi_{r}=45^{0}$, the measured values for side flow angles are higher than those from Colwell's model but significantly lower than the theoretically determined required side flow angle. In contrast, for $\psi_{r}=15^{0}$, as observed in Figure 4.5, the measured side flow angles for the UCB tool are consistently lower than both the required side flow angles and values from Colwell's model.

Results from the tests on AL 2024 for $\psi_{r}=45^{0}$ and $\psi_{r}=15^{0}$ are shown in Figure 4.6 and Figure 4.7 respectively. Measured side flow angles for both the Korloy insert and the UCB tool are plotted in the same graph. As mentioned previously in Section 4.2, no flat rake faced tools were used in the aluminum cutting tests due to excessive buildup on the cutting edge. From the results of the cutting tests on AL 2024, the following observations are noted:

1. The general trends for cutting tests on AL 2024 for both $\psi_{r}=45^{\circ}$ and $\psi_{r}=15^{0}$ are similar to those observed in the AISI 1018 tests.

2. For the Korloy inserts at $\psi_{r}=45^{\circ}$, it is observed that side flow angles seem to closely match values from Colwell's model, more so at higher depths of cut. This is in contrast to the AISI 1018 results, where Colwell's model tended to predict on the higher side for the baseline tools. This fact can be attributed to the effective positive inclination provided on the Korloy insert. However, when the lead angle is changed to $\psi_{r}=$ $15^{0}$, values from Colwell's model are higher. 


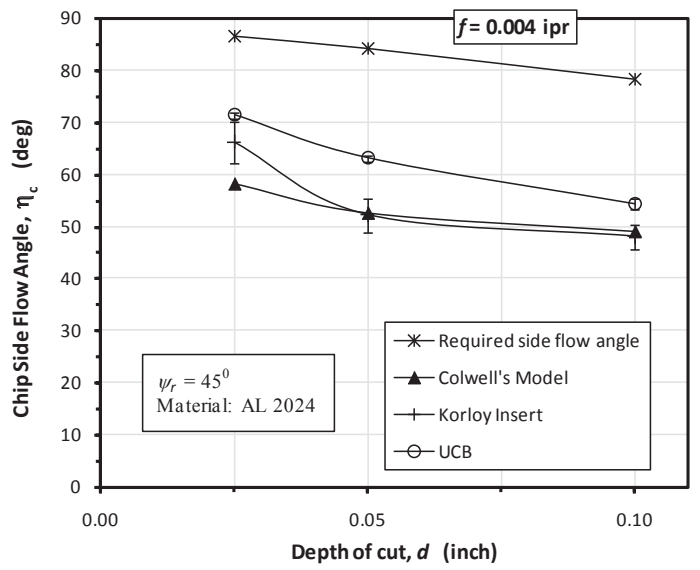

(a)

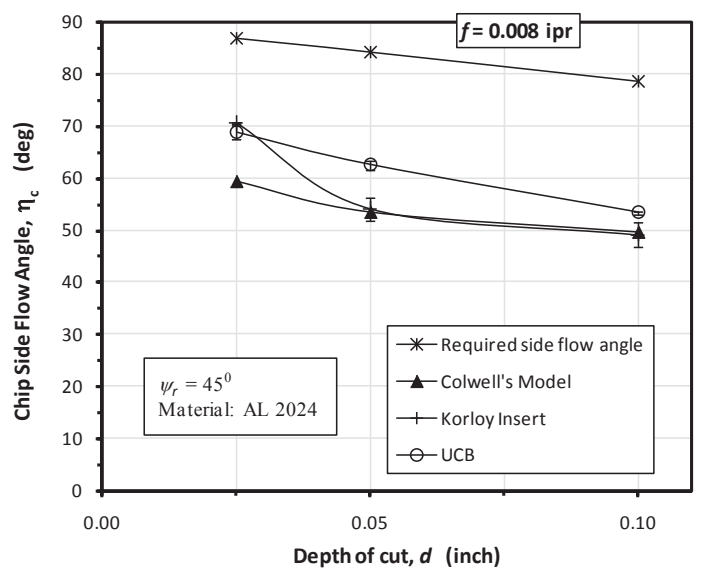

(b)

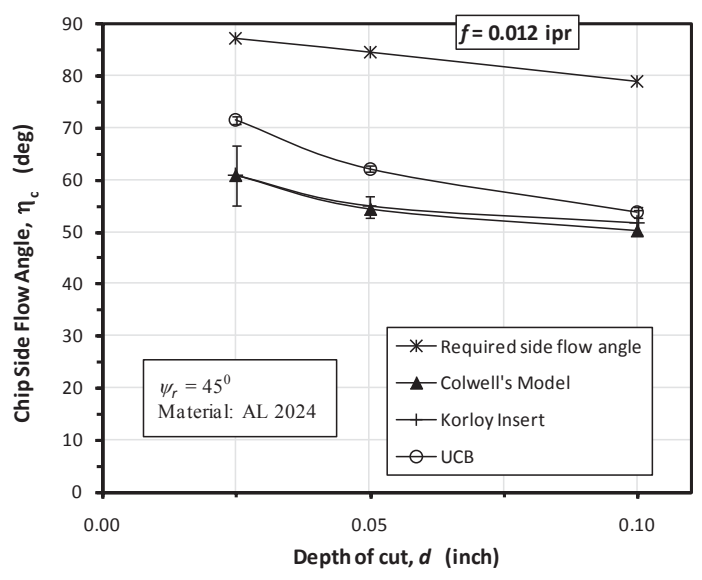

(c)

Figure 4.6: Measured chip side flow angles for AL 2024; $\psi_{r}=4^{0}$. 


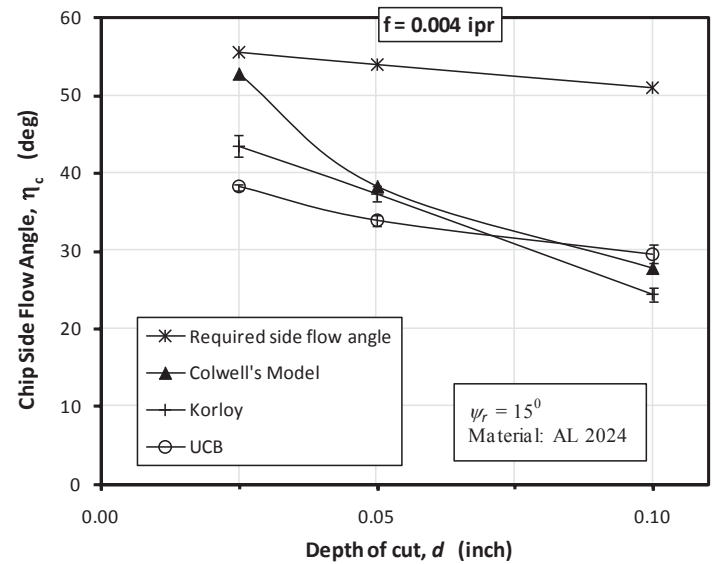

(a)

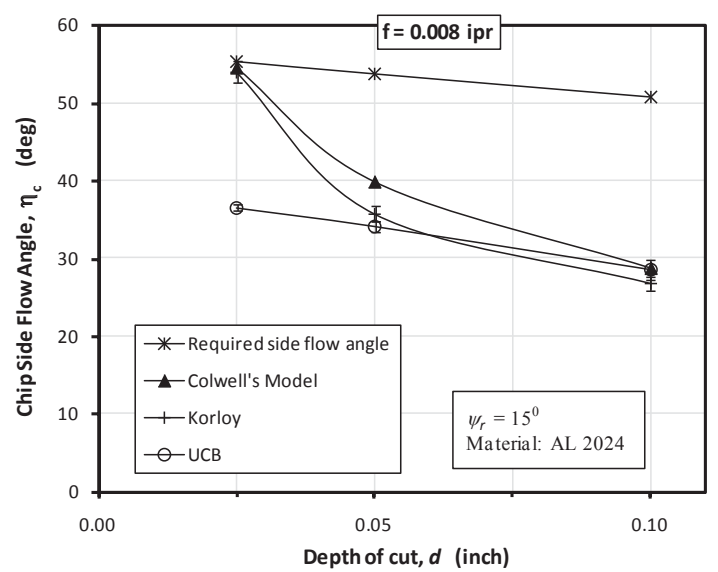

(b)

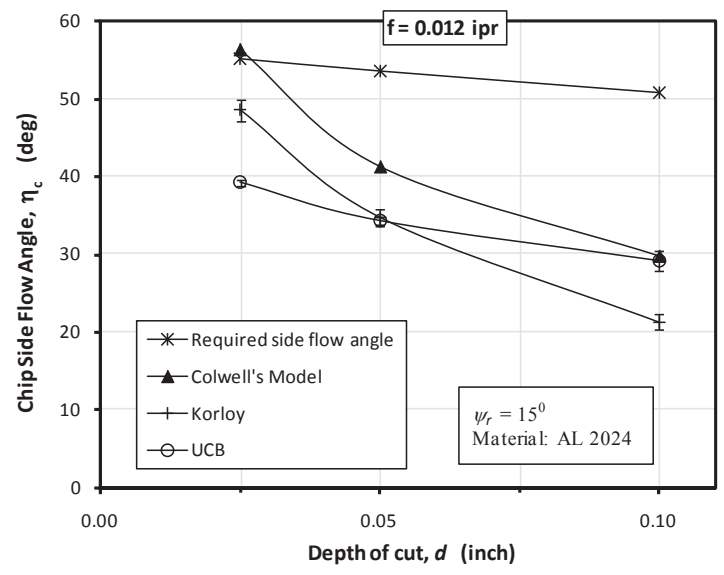

(c)

Figure 4.7: Measured chip side flow angles for AL 2024; $\psi_{r}=15^{0}$. 
3. For the UCB tool at $\psi_{r}=45^{\circ}$, the measured side flow angles are only slightly higher than the Colwell's values but again fail to match the values of the required side flow angle. At $\psi_{r}=15^{\circ}$, however, an interesting result can be observed. At low depths of cut, the side flow angles are lower than the Korloy insert whereas a cross over is seen as the depths are increased.

\subsection{Discussion of Results}

\subsubsection{The Side Flow Angle and Stabler's Rule}

Since the results presented in the previous section for both AISI 1018 steel and AL 2024 display similar trends, the discussion hereon, unless otherwise mentioned, will be limited to the case of AISI 1018.

The results from both the flat faced tool and Sandvik inserts follow established knowledge on chip flow characteristics. That Colwell's model tends to over-predict the natural side flow angle has been discussed by Spaans (1970) in his experiments on cutting with a corner radius tool. The lower values generally seen in experiments are attributed to the effects of friction that are not accounted for in Colwell's method, which is purely geome-

try based. A more sophisticated method to better predict the chip side flow angle would be to utilize a mechanistic based approach (for e.g., the Subramani or the Endres model as discussed in Chapter 2) that accounts for friction via the cutting forces. However, Colwell's model tends to be more widely used as a benchmark in chip flow studies since it is relatively easy to implement. 
For the UCB tool, the results of chip flow angles present some interesting details. Firstly, the lower side flow values than those of Colwell's model as seen in the case of $\psi_{r}$ $=15^{0}$ (Figure 4.5 and Figure 4.7) is surprising. Normally, when cutting with small lead angles and, for a given insert geometry, due to higher end cutting angles, the chip side flow angle tends to increase due to the added effect of the minor cutting edge as evidenced by Nedess and Hintze (1989) and Balaji (2000). In Figure 4.8, Nedess and Hintze's illustration of the chip contact zones shows a change in the effective chip side flow angle due to the added effect of cutting with the end cutting edge.

In the context of the UCB tests at small lead angles, an interesting question that arises pertains to the chip flow around the corner radius region. Given that in the UCB, the in-

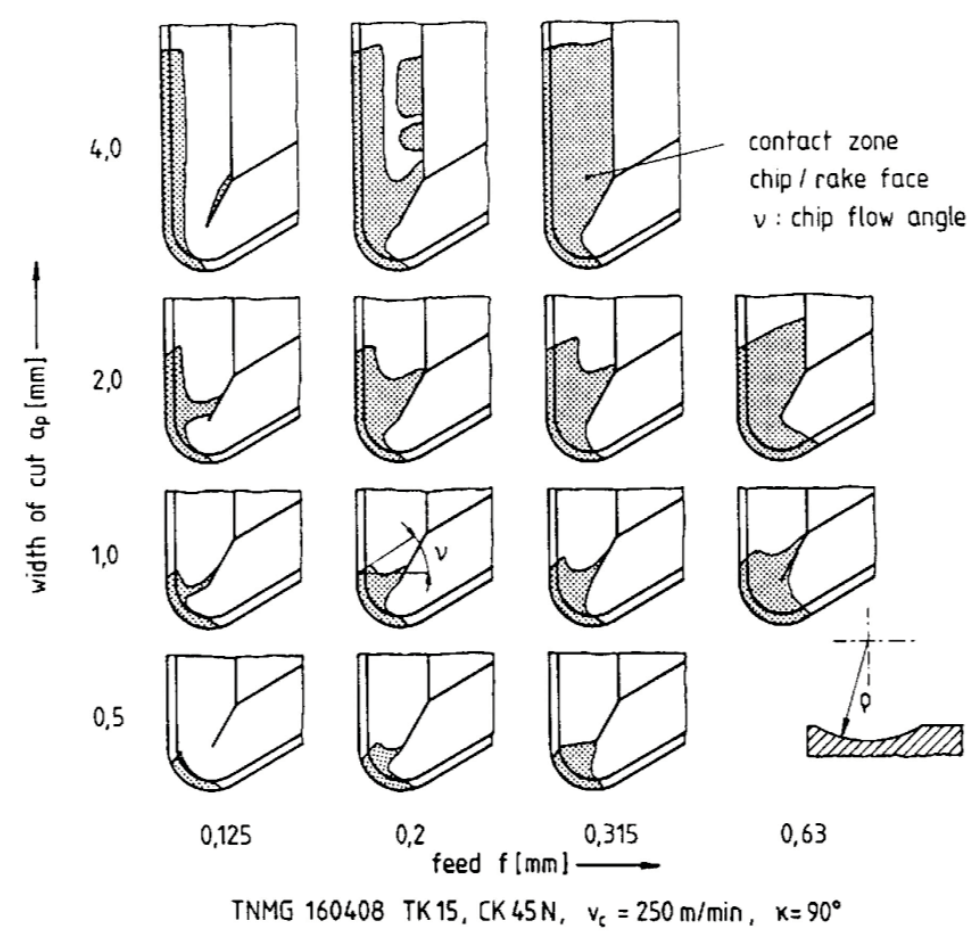

Figure 4.8: Tool-chip contact regions with variation in cutting parameters. (Nedess and Hintze, 1989) 
clination angle (drop) is symmetric about the corner radius, would the geometry cause the chip to wrap around the bi-directional edge drop? To further investigate this effect, cutting tests for the UCB tool at $\psi_{r}=15^{0}$ were conducted with the rake face dyed blue. The observed chip contact is shown in Figure 4.9. From the figure, it is apparent that the chip does not just flow over the highest point at the corner radius region but rather overflows onto the symmetrical inclination of the minor cutting edge. Thus, it can be concluded that the inclinations on both the major and minor cutting edges being opposite in their sense of direction results in a conflicting effect on the side flow angle; the increase in side flow on the major cutting edge is tempered by the inclination of the minor cutting edge

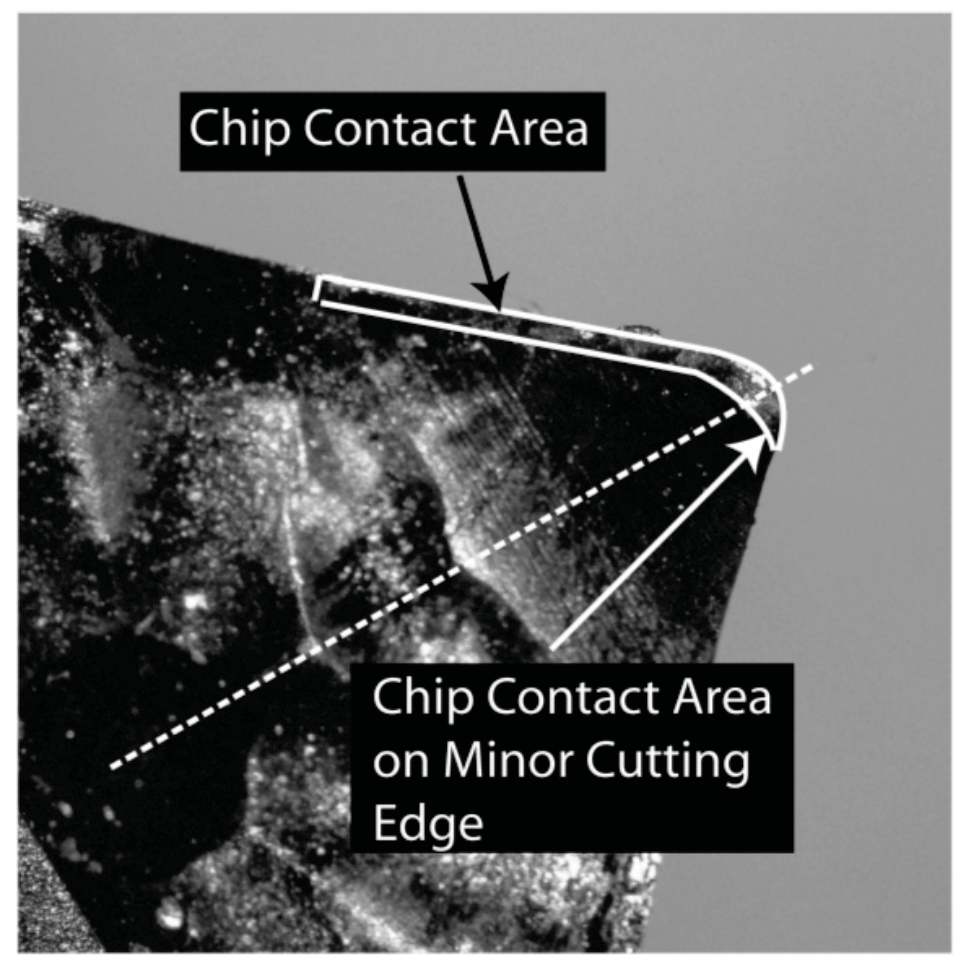

Figure 4.9: Photograph showing extension of tool-chip contact zone onto minor cutting edge for UCB tool. Work material: AISI 1018; $\psi_{r}=1^{0}$. 
resulting in a side flow angle lower than that given by Colwell's model. Clearly, the incongruity of the side flow angle seen for the UCB tool at $\psi_{r}=15^{\circ}$ is a result of the unique geometry of the UCB tool and its effects when the minor cutting edge is invoked in the cutting process.

The second and rather more important observation is that, the side flow angles seen in the UCB tests are significantly lower than the theoretically required flow angle for the chip to reach the target location. It may be recalled that one of the inherent assumptions of the model was that Stabler's rule is satisfied in designing the elemental edge inclination angle required to deviate the chip flow from its natural flow direction. In Figures 4.4 - 4.7, the difference in the side flow angle between Colwell's model values and the required side flow angle, in effect, represents the added side flow to satisfy the design objective - that of guiding the chip to the target location - and forms the component based on Stabler's rule. The large discrepancy observed in the experimental results certainly needs closer examination.

As discussed in Chapter 2, Stabler's rule has been divisive in its application to the chip flow in oblique cutting, especially when using large inclination angles. Note that in actual practice, the inclinations are of constant value and are rather low. As such, the mismatch in Stabler's rule with the actual chip flow is considered to be of academic interest. But in the context of the UCB tool with its large inclination angles, the deviations pose strong repercussions and need to be accounted for since the objective is to use the inclination angle to direct the chip flow to a fixed target location.

In the past, several researchers have questioned the validity of Stabler's rule when applied to large inclination angles. As shown in Figure 4.10, Brown and Armarego 
(1964) proved the inadequacy of Stabler's law at higher values of inclination angle. They argue that the chip flow angle is independent of the inclination angle and suggest that the normal rake angle is more relevant for determining the chip flow angles. Brown and Armarego's results indicate that the deviations in Stabler's rule could be as high as 30\% or greater depending on the normal rake angle. Note that in Brown and Armarego's work, the constant inclination angle as well as the specific normal rake angle was maintained by manipulating the side and back rake angles. Given the findings of Brown and Armarego, it appears that the present model would need further augmentation to better account for the deviations observed. But whether the inclination angle is used or the normal rake angle, reducing the large deviation seen in the UCB tests would necessitate incorporation of an added inclination to the existing value. The resulting tool edge would then possess ex-

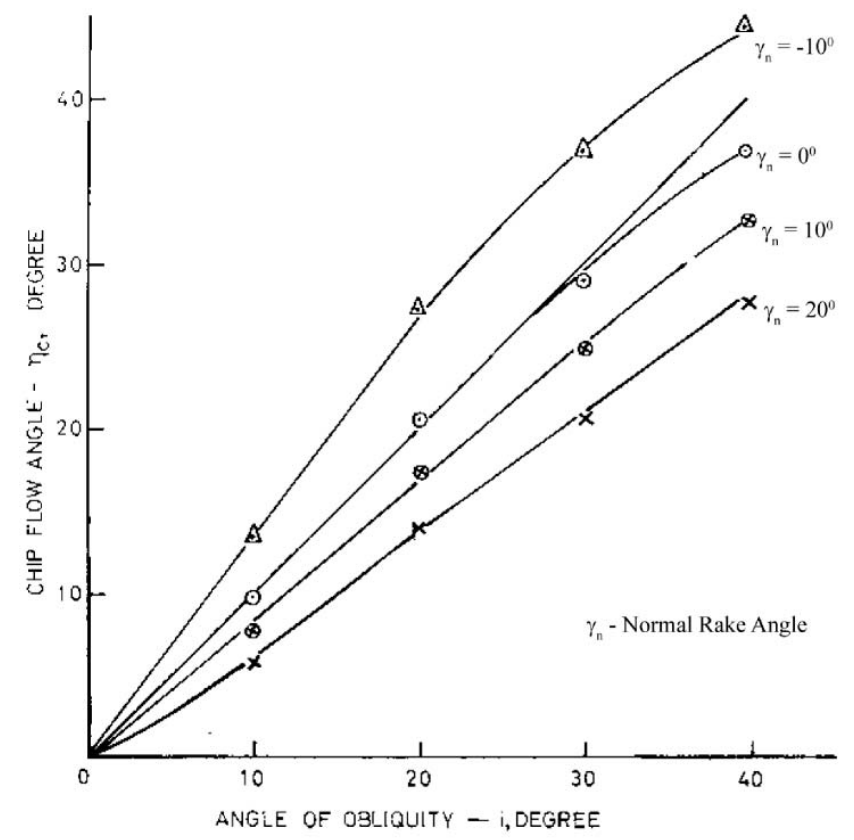

Figure 4.10: Deviations of Stabler's rule. (Brown and Armarego, 1964) 
tremely high inclination angles - values which are physically improbable to be used in metal cutting.

On the other hand, Hu et al. (1986) observe that that the deviation of Stabler's rule is significant only at higher feeds and reason that the mismatch seen is due to the fact that the actual plane of chip flow and the viewing (tool reference plane) plane are different. The difference in viewing planes is very much the case for the UCB since the camera setup was normal to the tool reference plane. One solution around this problem is to simply tilt the camera so that the focus is on the plane of the chip flow (Russell and Brown, 1966; Li et al., 1997). However, the constantly varying edge inclination on the UCB tool renders this technique moot. Hu et al. (1986), proposed an equivalent cutting edge method as shown in Figure 4.11, which, when used with subsequent transformations seemed to show better correlation with Stabler's rule. Note that the equivalent cutting edge me-

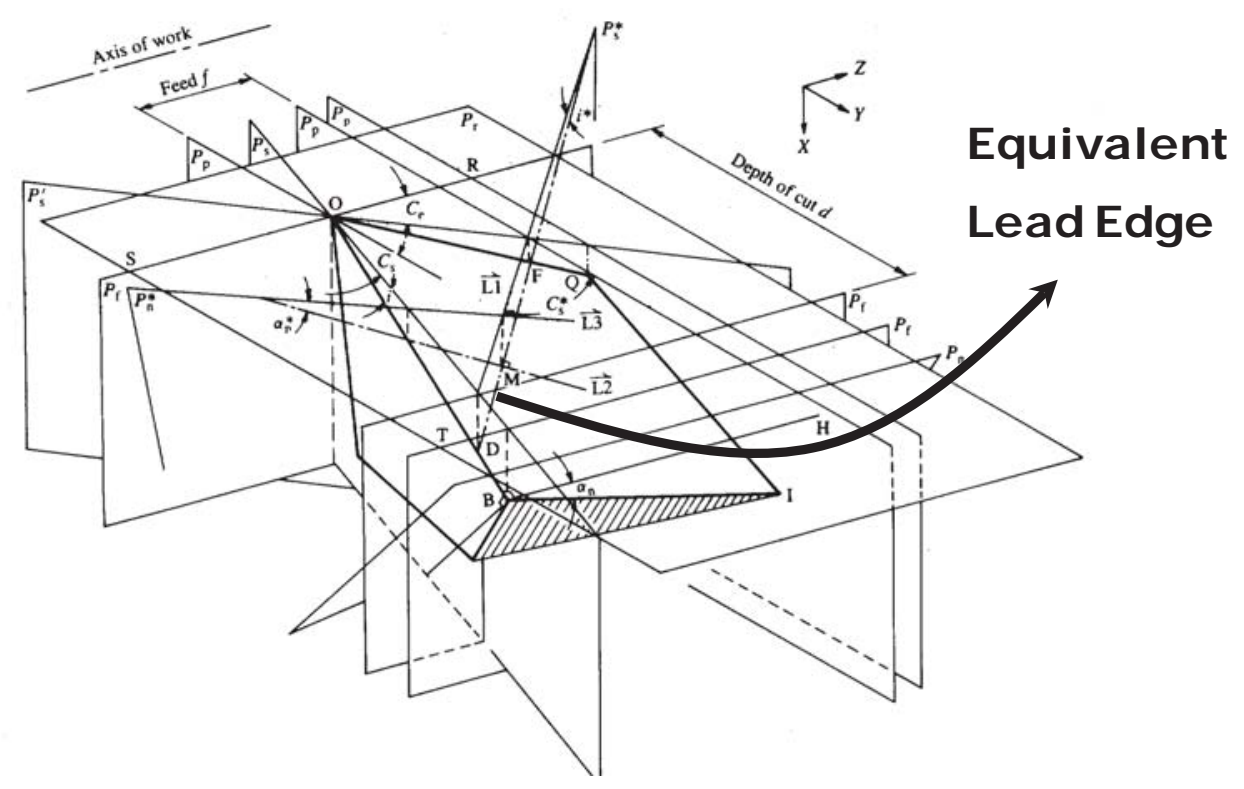

Figure 4.11: The equivalent cutting edge for oblique cutting. (Hu et al., 1986) 
thod of Hu et al. considers a straight cutting edge with an inclination angle provided via the tool holder. As such, it is relatively straightforward to apply the equivalent cutting edge transformation method. Clearly, direct application of an equivalent cutting edge on the UCB tool is impossible due to the varying edge inclination as well as the absence of a flat rake face; the rake face is eliminated to create a highly restricted contact surface which makes the judgment of the plane of equivalent edge difficult. However, an attempt was made to investigate the possible implication of using a transformation to the measured side flow angles for the UCB tool. In its application to the UCB, the inclination of the equivalent cutting edge is considered by joining the Z-depths of the edge points corresponding to the respective feed and depth combination. Figure 4.12 shows the measured side flow angle transformed onto the chip flow plane for the specific case of $\psi$ $=45^{0}, f=0.005$ ipr. It can be observed that transforming the measured chip flow angle onto the actual plane of chip flow only results in a marginal increase $\left(\sim 9^{0}\right)$ at higher depths of cut, which is expected due to the higher inclination along the tool edge as the depth of cut increases. However, the chip side flow angles still fall considerably short from the required side flow angle. It is acknowledged that this technique is only an approximation at best. However, the equivalent cutting edge method still presents the best technique to observe the effects of measurement errors resulting from the visual perception.

Yet another hypothesis to explain the inconsistencies of Stabler's rule is offered by Shaw et al. (1952), who state that "chip flow direction is not simply a matter of geometry", but includes other considerations - mainly friction from chip tool contact. Their results indicate that the chip side flow changes with use of coolant ( $\eta_{c}$ increases) as well 


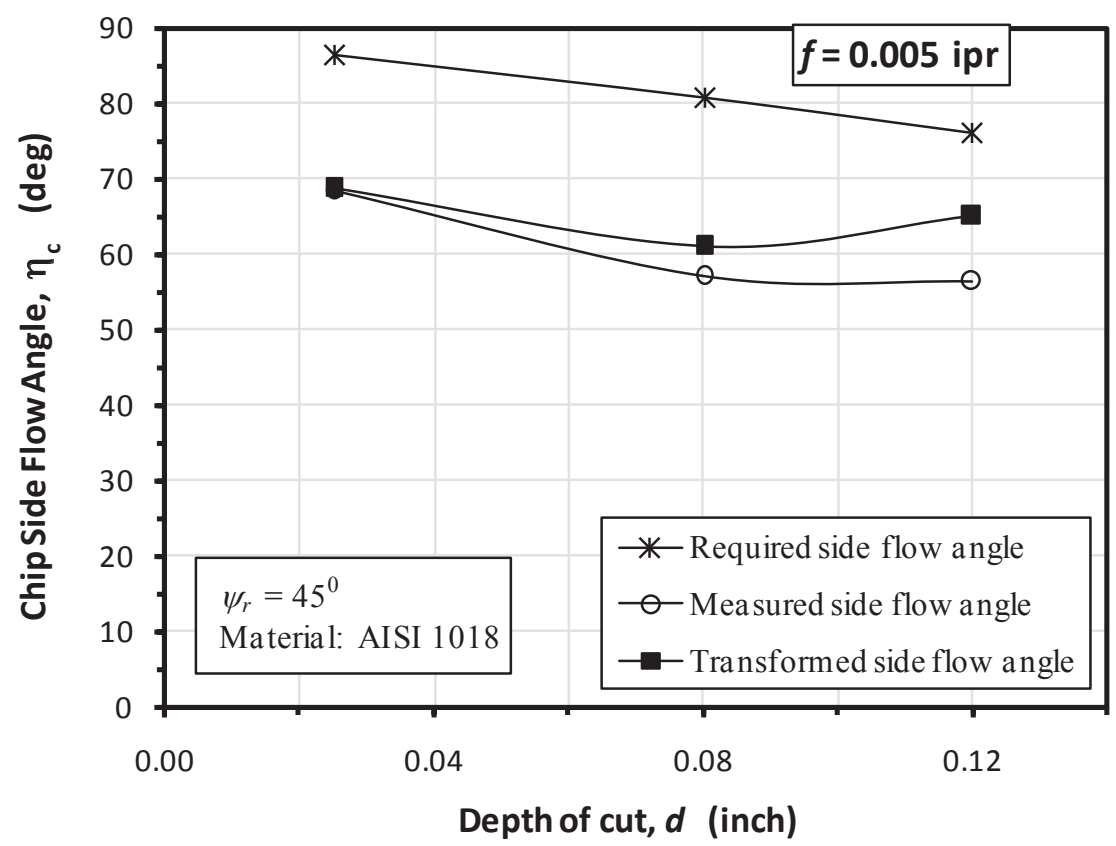

Figure 4.12: Transformed chip side flow angles using the equivalent cutting edge method; Work material: AISI 1018, $\psi_{r}=4^{\circ}, f=0.005$ ipr.

as the friction properties of work material (in tests by Shaw et al., $\eta_{c}$ decreased for aluminum as compared to steel) itself. More recently, Seethaler and Yellowley (1996) used a mechanics based model with the effects of friction to explain the deviations observed in Stabler's rule. Interestingly, their model uses the normal rake angle as was proposed by Brown and Armarego (1964). As shown in Figure 4.13, results from their numerical model shows that Stabler's rule is satisfied only under frictionless conditions. When friction was considered as a variable, the deviation between inclination angle and side flow angle increased to $>50 \%$ even under relatively low inclination angles of $15^{\circ}$. Seethaler and Yellowley concluded that friction and positive rake angles decrease the side flow angle. 


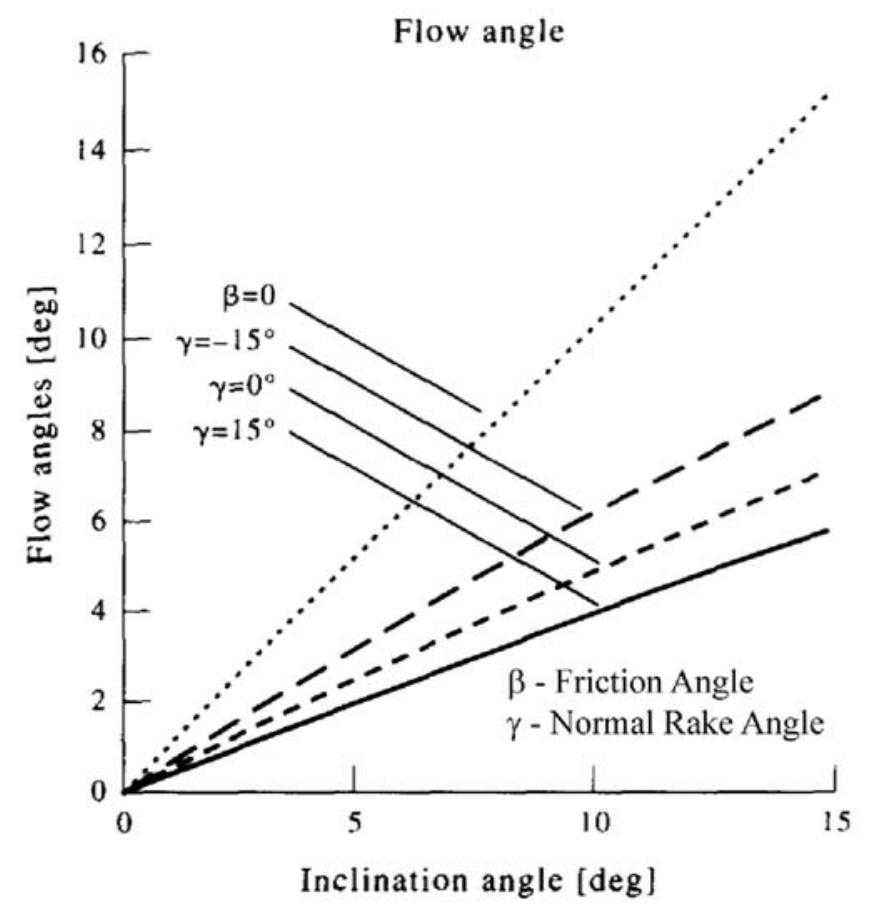

Figure 4.13: Seethaler and Yellowley's numerical model showing deviations of Stabler's rule due to friction. (Seethaler and Yellowley, 1996)

Considering the fact that the rake face is eliminated in the UCB tool, the effects of friction can be assumed to be negligible. A more important consideration however, is the possible restriction to the chip side flow due to the tool-chip contact zone at the end of the inclination angle (especially at high depths of cut). To assess the effects of tool-chip contact on the side flow angle, additional cutting experiments were conducted using the UCB tool. The tool-chip contact region was identified by bluing the rake face and determining the wear mark from the chip flow as shown in Figure 4.14. AL 2024 was used as work material since the chip contact marks were more prominent and easier to observe. Tests on AISI 1018 steel also returned a similar tool-chip contact pattern. An extended 


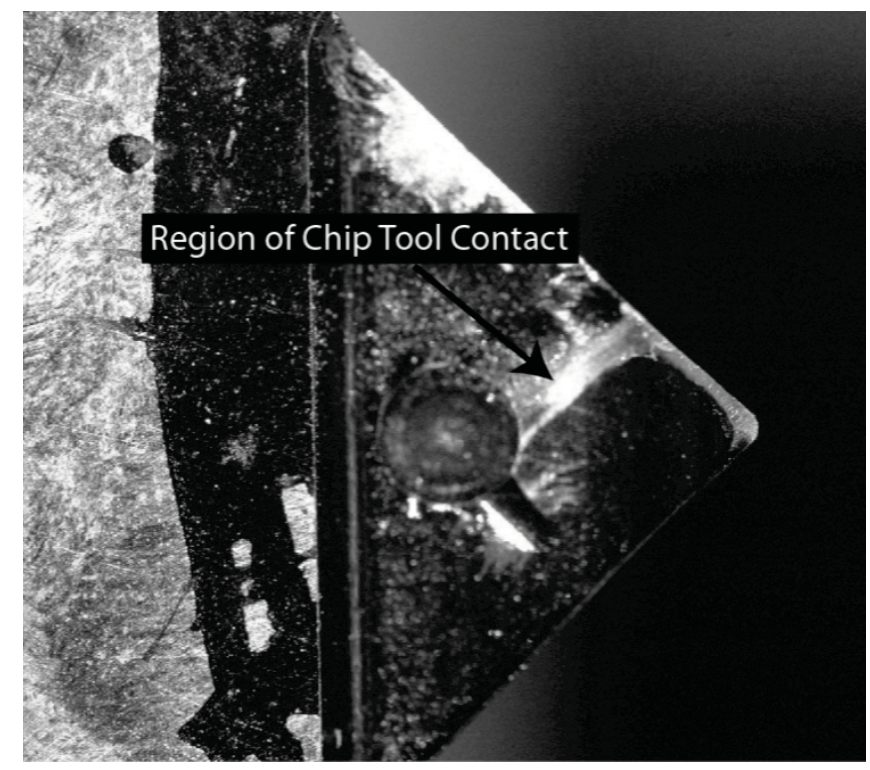

Figure 4.14: Typical tool-chip contact surface for UCB tool; Work material $=\mathrm{AL} \mathrm{2024}, \psi_{r}=4^{\circ}, d=0.100 \mathrm{inch}, f=0.012 \mathrm{ipr}$.

tool-chip contact zone on the restricted tool rake face is seen to occur on the outer edge of the chip. Since this extended contact between the tool-chip pair could potentially restrict the side flow of the chip, a 0.040 " pocket was milled on the rake face in order to limit the contact zone to a minimum. Results of the tool chip contact tests are shown in Figure 4.15 for the case of $\psi_{r}=45^{0}, d=0.100$ inch and $f=0.012$ ipr. Based on the cutting tests, no significant change in side flow angle was observed. Further, it was also observed that no contact occurred between the chip and the bottom of the pocket indicating that the chip flow was essentially "in air" over the tool rake face. Based on results of the last set of experiments, it is concluded that the deviations observed are a result of the tool geometry alone. Further work is required to better quantify the chip side flow issue. 

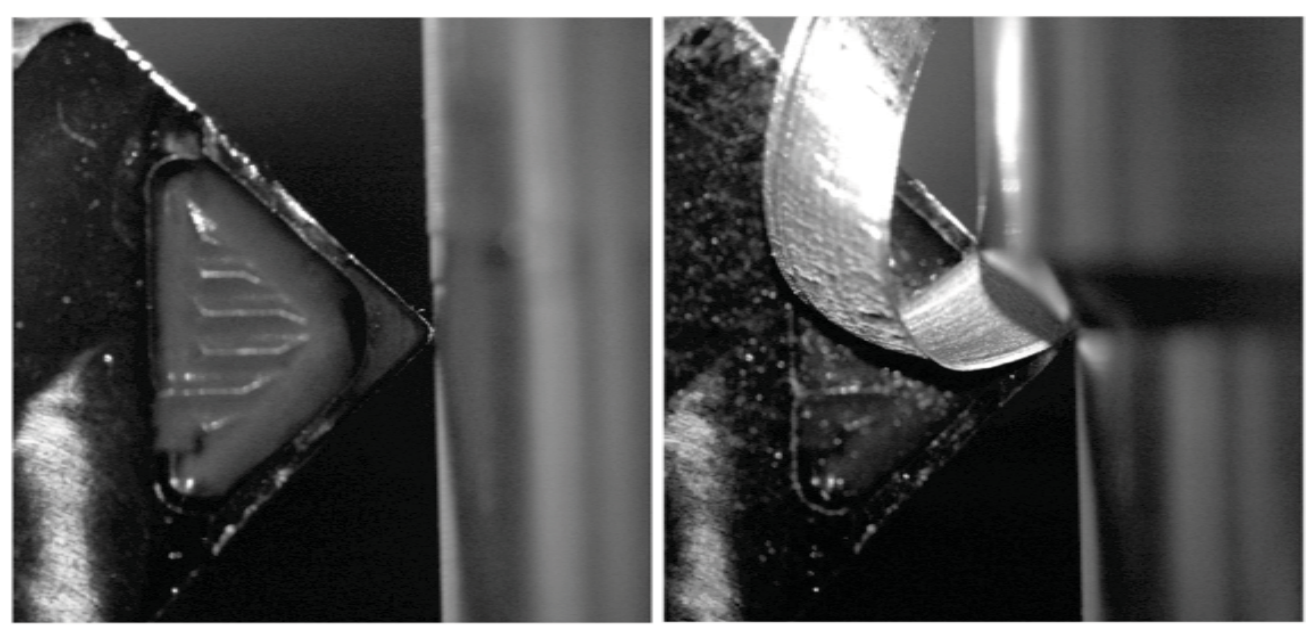

Figure 4.15: Photograph showing unchanged chip flow angle with rake face eliminated; Work material $=\mathrm{AL} \mathrm{2024}, \psi_{r}=45^{\circ}, d=0.100$ inch, $f=0.012 \mathrm{ipr}$.

\subsubsection{A Qualitative Assessment of Chip Morphology}

Given that chip flow includes components of both side flow as well as curl (both up and side), a study of the chip behavior in its entirety is also required to fully understand the action of the UCB geometry. A qualitative assessment of the chip characteristics is presented in this section. Chips produced using the UCB tool are compared with those from the commercial baseline inserts with standard chip breaker designs.

\subsubsection{Chip Flow}

The discussion on qualitative assessment for chip flow pattern is confined to AISI 1018 steel since the general chip flow characteristics for both the work materials are similar. Moreover, chip morphology from the flat rake faced inserts are ignored since flat tools with no chip breakers are generally not used in the context of chip control. Addi- 
tional photographs from the tests on the flat rake faced tool, as well as replicated tests for AISI 1018 steel and all replicated tests for AL 2024 are included in APPENDIX E for reference.

For the UCB tool and Sandvik insert, the same representative photographs used for the measurement of side flow angles are shown as functions of feed and depth of cut in Figures $4.16-4.17$. From the photographs, several observations are noted. Foremost, from Figure 4.16, for the Sandvik insert at $\psi_{r}=45^{0}$, it is seen that the chip flow is inconsistent at best even within the recommended range of feed and depth of cut $(f=0.006$ $0.017 \mathrm{ipr}, d=0.028-0.142$ ). Given that the chip breakers are designed to guide chips to break in one of the several modes proposed by Nakayama (1962), it can be seen that only a few of the recommended cutting conditions actually guide the chip to the work shoulder or, over the tool edge to the flank region. The only condition where the chip breaker appears to break the chip as intended is at the condition $d=0.120$ inch and $f=0.008 \mathrm{ipr}$ while the conditions $d=0.120$ inch, $f=0.005 / 0.014$ ipr and $d=0.080$ inch, $f=$ 0.005/0.014 ipr could, arguably, have a potential chip breaking cycle associated with them. In most other cases, the chip takes on a tight helical curl and entirely misses the intended obstruction. Several other researchers, most notably Jawahir (1988) and Jawahir and Oxley (1988), have also noted the ineffectiveness of chip breakers within their recommended cutting conditions. In fact, a variety of flow patterns can be observed even while the chip maintains a curled helical form. At low depths and feeds the chip attains a tight curl with the helix axis parallel to the tool edge resulting in an unbroken chip. When breakage did occur, it was more a result of the chip meeting an obstruction such as the tool holder or the tool post. The most obvious reason for the unbroken chip is the 


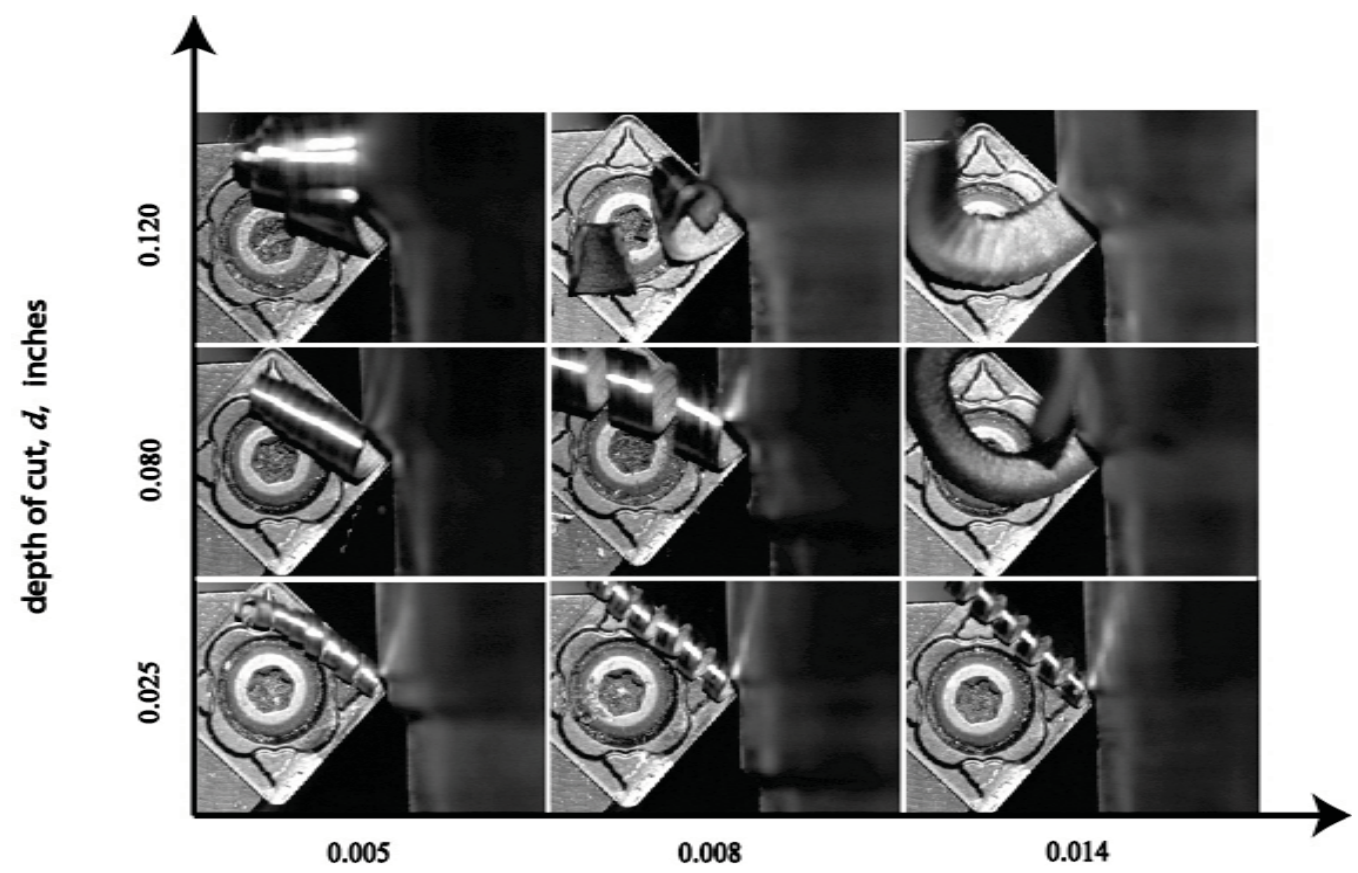

feed, $f$, ipr

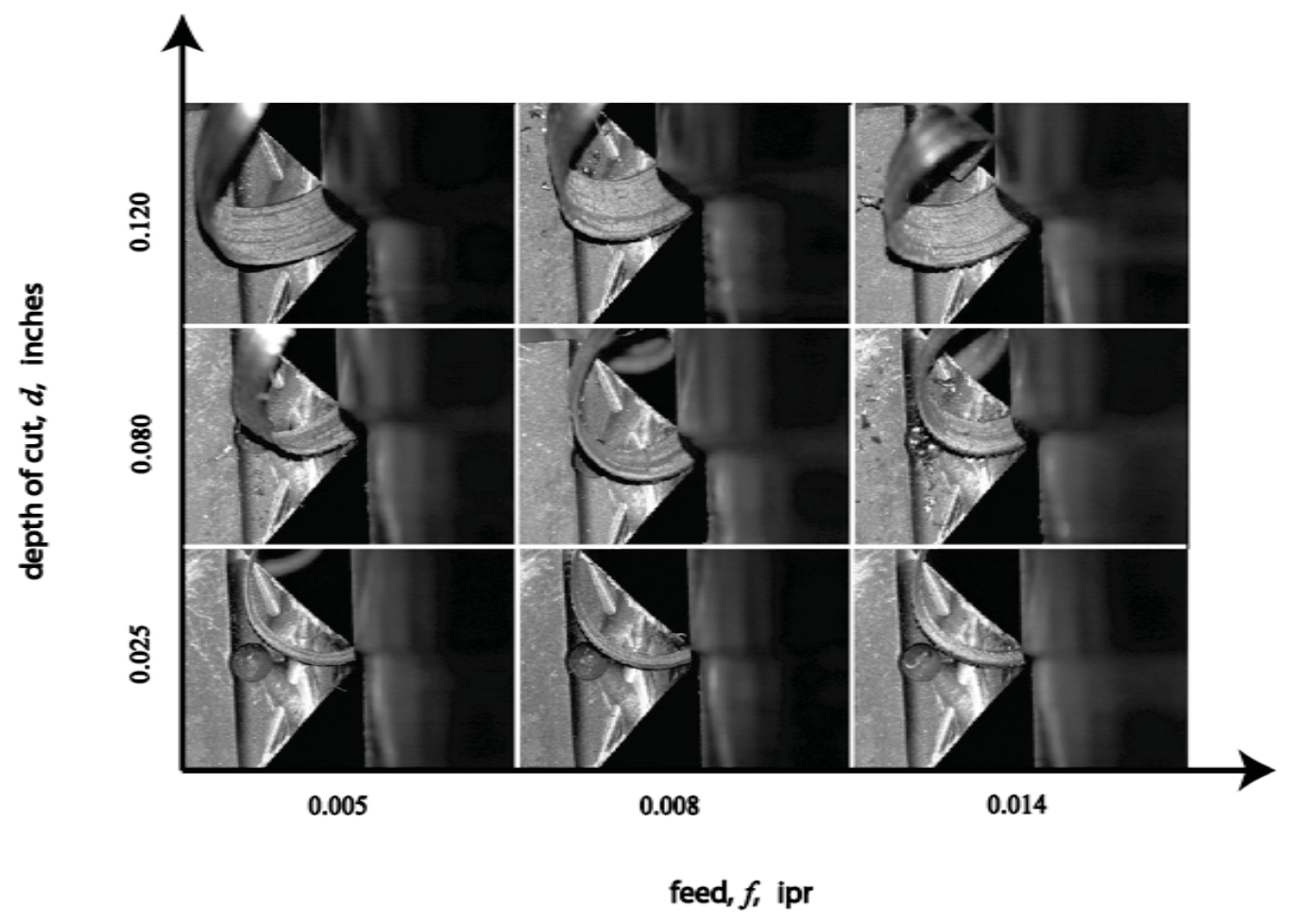

Figure 4.16: Chip flow patterns for: (top) Sandvik SCGT-433 insert (bottom) UCB tool; Work material $=$ AISI 1018, $\psi_{r}=4^{\circ}$. 


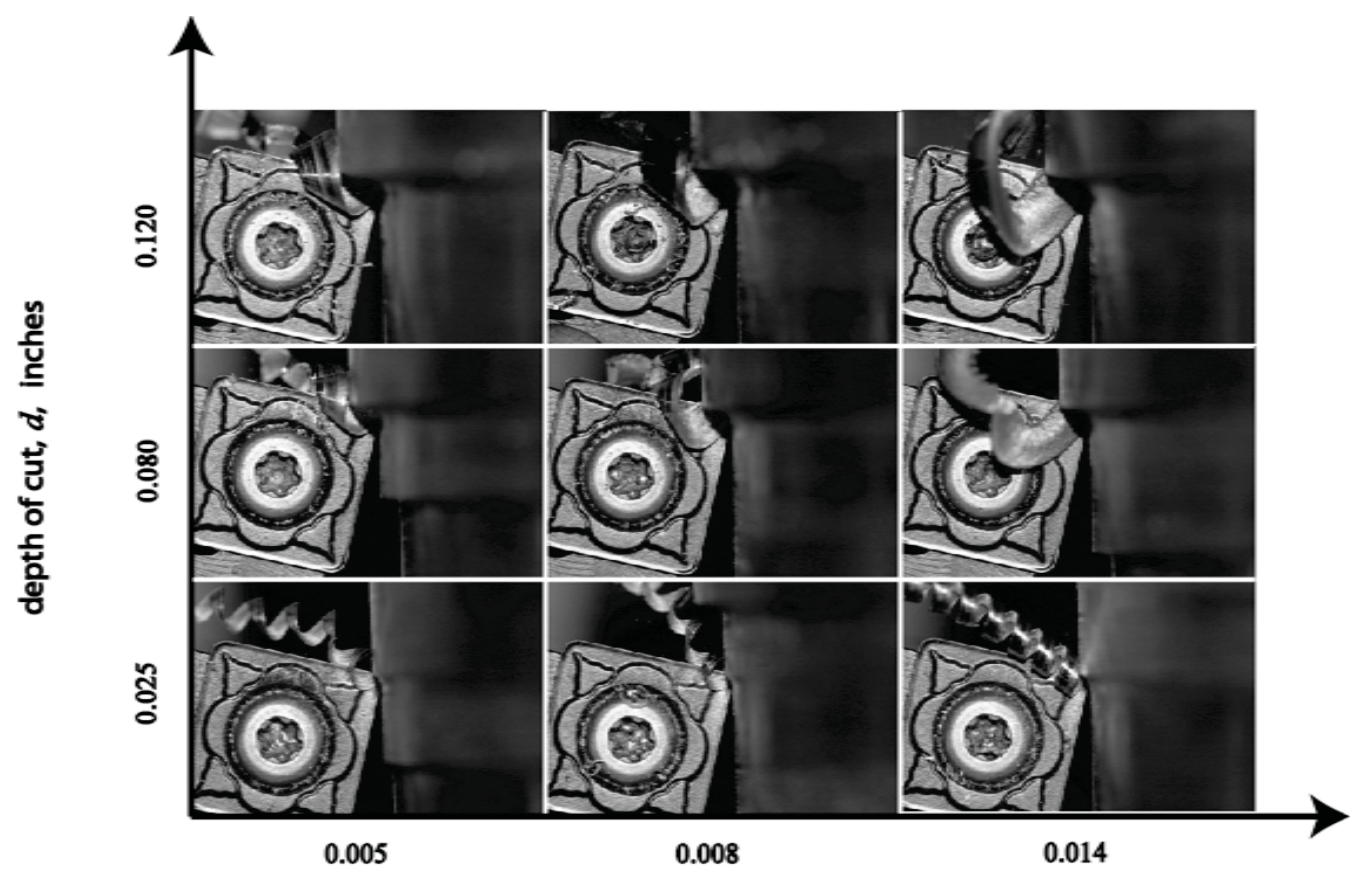

feed, $f$, ipr

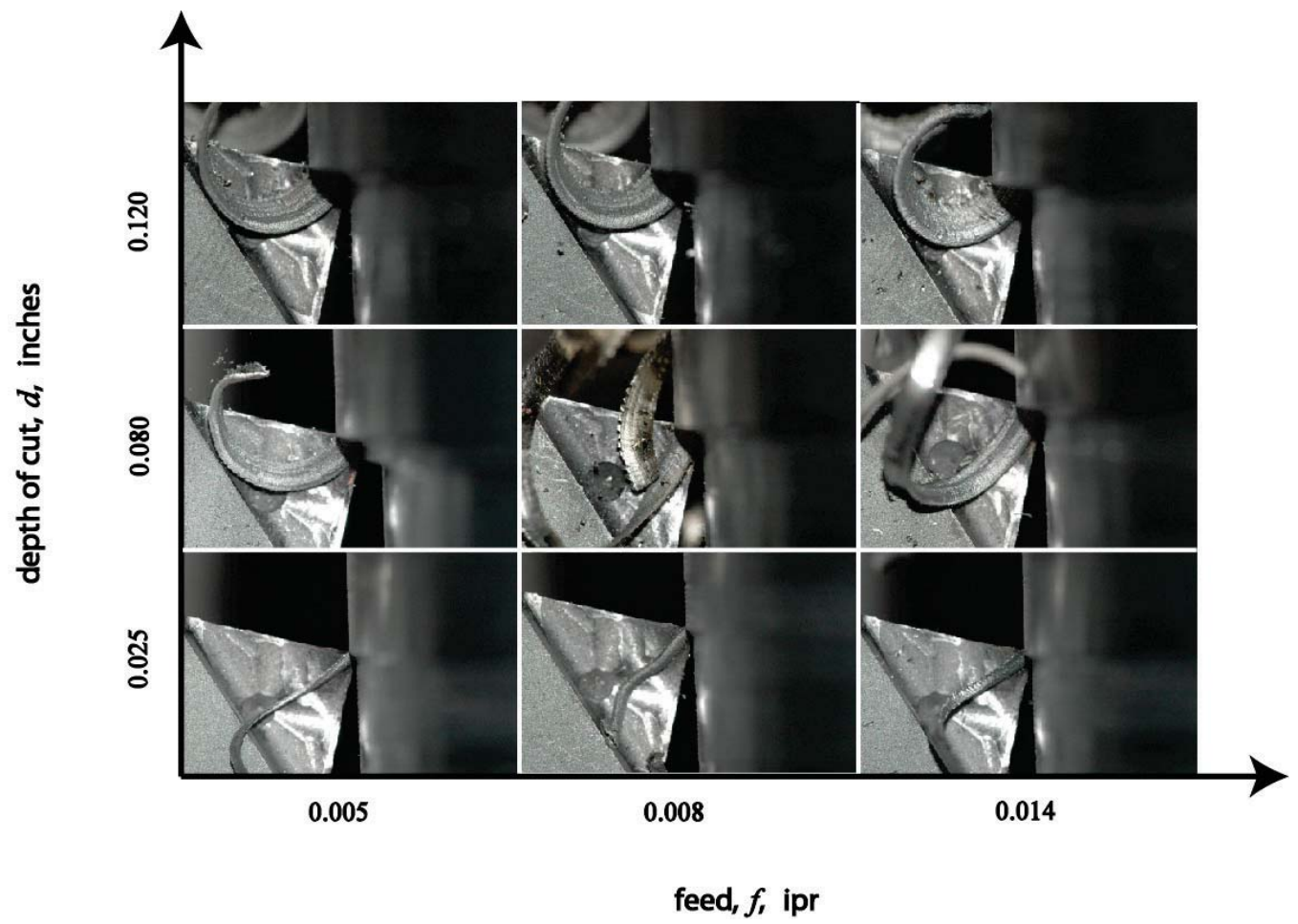

Figure 4.17: Chip flow patterns for: (top) Sandvik SCGT-433 insert (bottom) UCB tool; Work material $=$ AISI 1018, $\psi_{r}=1^{0}$. 
placement and height of the chip breaker design - the breaker is simply not close enough to the cutting edge or high enough to curl the chip over the tool edge to anchor on the tool flank. A more dramatic change can be seen to occur at the high depth/feed (in particular, $d=0.120, f=0.014$ ipr) combination. The chip form changes abruptly with the helix axis now normal to the rake face plane. At these conditions, which are still well within the recommended chip breaking parameter values, the chip breaker ceases to operate and the chip deflection observed is due to the projecting screw fastening the insert to the tool holder.

From Figure 4.17, a slightly different pattern emerges at $\psi_{r}=15^{0}$, with the difference being that the chips flow over the cutting edge and toward the intended breakage point more consistently. While it was observed that the chip breakability increased at higher depths, the low depth conditions still present a condition wherein the chips are not broken as per the design intent of the chip breaker, but more due to self-weight of the chips or due to other external obstructions. It can also be seen that the chip breaker action is again absent at high feed/depth values.

By contrast, chip flow for the UCB tool is more consistent in nature. The consistency in chip form when cutting with restricted contact tools has been observed in the past. Klopstock (1925), in his experiments with the first recorded restricted contact tool, notes the drastic change in chip forms observed when the rake face of the tool is eliminated. A similar observation was noted in the work of Chao and Trigger (1963), where the restricted contact of the tool results in a transformation of the chip form from straight to a curled helix. More recently, Jawahir (1988) presented similar findings in his work on restricted contact tools. One very surprising aspect seen in the photographs of chip flow for 
the UCB tool is that despite the deviations observed in the side flow angle, the chip flow consistently reaches the target location due to the added contribution of the side-curl. In hindsight, the fact that the unintentionally chosen target location perchance happened to be at the center of the tool coordinates was rather fortuitous. Note that the consistency extends over multiple levels of lead angle, work material and process parameters (refer APPENDIX E). The variations seen at the low depth cases for $\psi_{r}=45^{0}$ (and, for $\psi_{r}=$ $15^{0}, d=0.080$ inch, $f=0.008$ ipr) are probably due to the small material projection left behind by the machining of the rake face. One important observation from the flow patterns is that, the dominant side-curl orients the chip width differently relative to the target location. The chip orientation can be seen very clearly in the results from the cutting experiments using AL 2024 (refer APPENDIX E), wherein the material projection on the rake face mentioned earlier was eliminated.

\subsubsection{Chip Form}

Apart from the chip flow itself, the chip morphology a posteriori is also commonly used to gauge the effectiveness of chip breaker geometries (Sandvik, 1996). Likewise, chips produced in the present cutting tests are studied using chip charts as shown in Figures 4.18-4.21. Chips collected from each cutting test are plotted as a function of feed and depth of cut. Due to the different nature of chips for AISI 1018 and AL 2024, both results are presented in this section. All forms are assessed in accordance with the definitions of chip forms under the standard ISO 3685:1993. Note in advance that the UCB tool is not fitted with the chip breaker system (discussed next in Chapter 5) and so chip breaking is not expected for the UCB tool. 

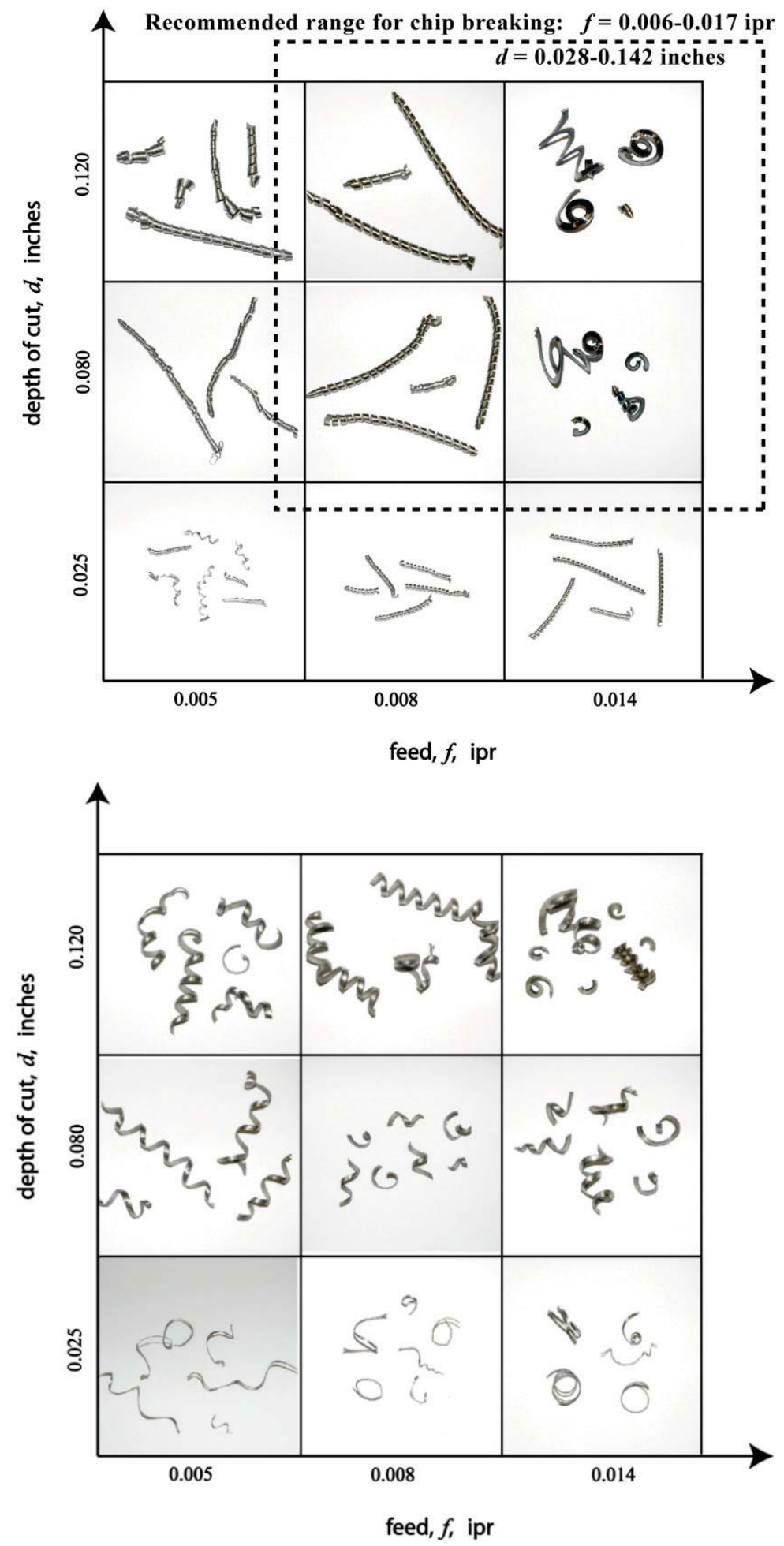

Figure 4.18: Chip morphology for: (top) Sandvik SCGT-433 insert (bottom) UCB tool. Work material $=$ AISI 1018, $\psi_{r}=4^{\circ}$. 

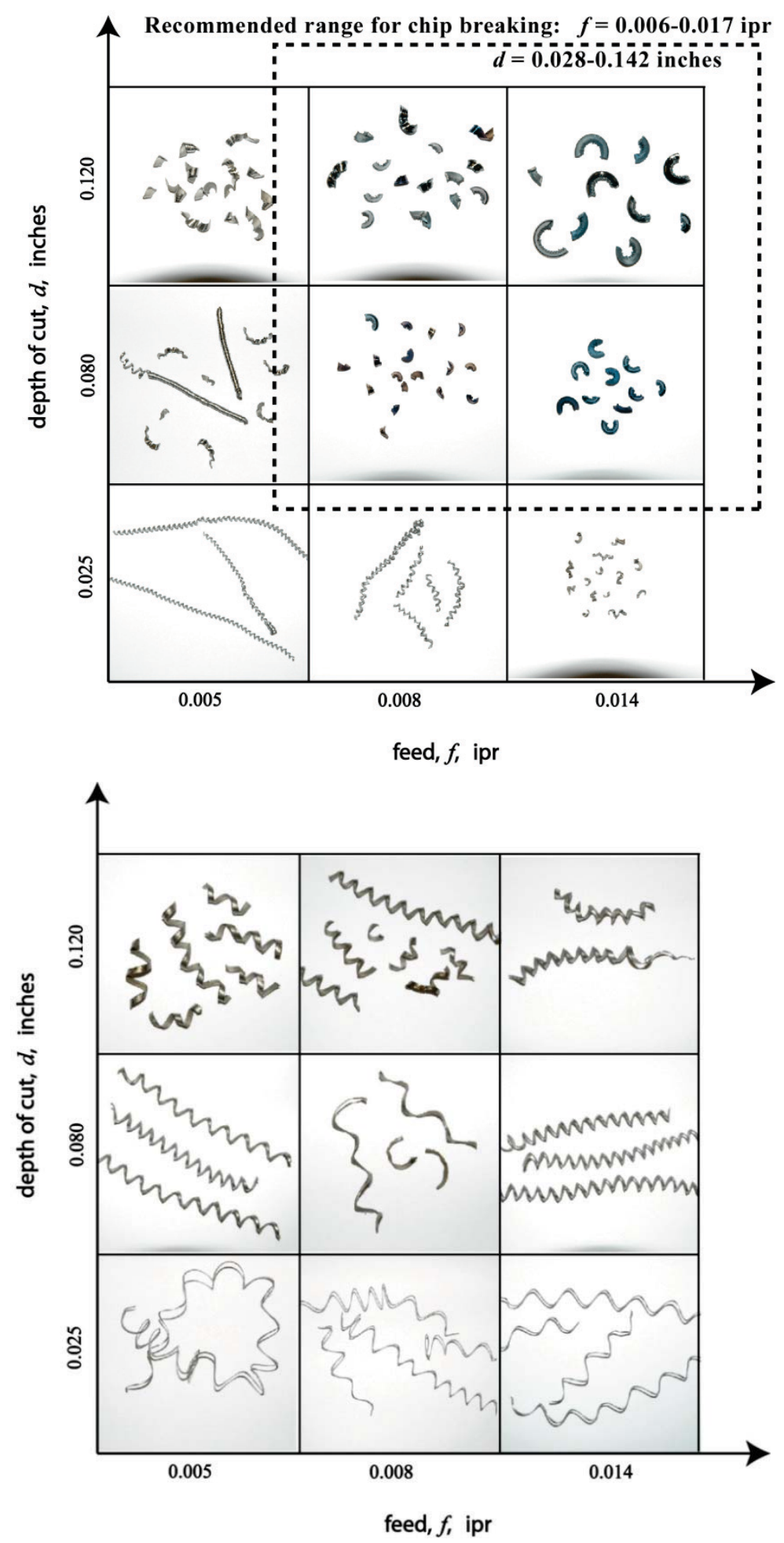

Figure 4.19: Chip morphology for: (top) Sandvik SCGT-433 insert (bottom) UCB tool. Work material $=$ AISI 1018, $\psi_{r}=\mathbf{1 5}^{\mathbf{0}}$. 

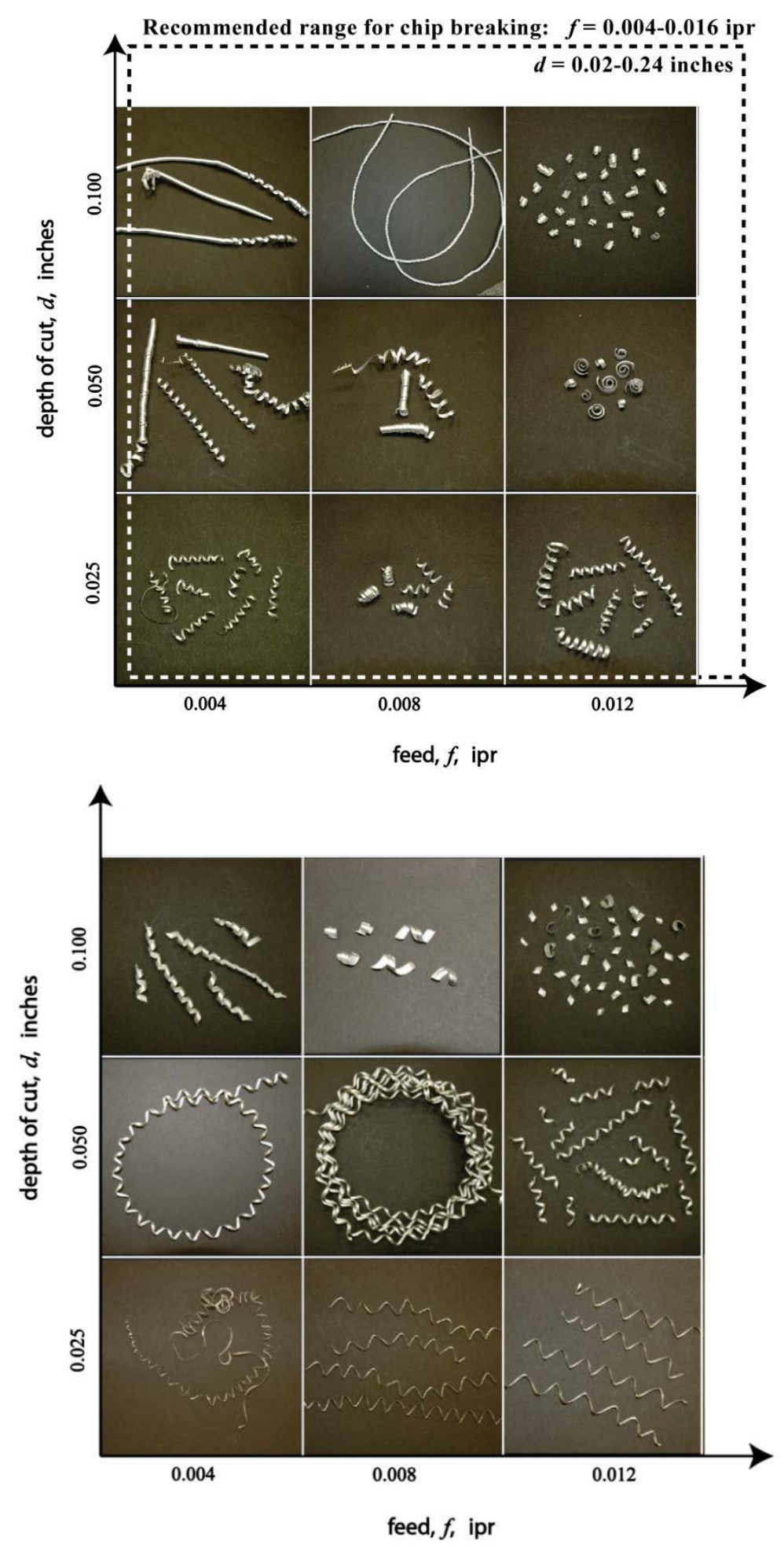

Figure 4.20: Chip morphology for: (top) Korloy SCGT-432 insert (bottom) UCB tool. Work material $=$ AL 2024, $\psi_{r}=4^{\circ}$. 

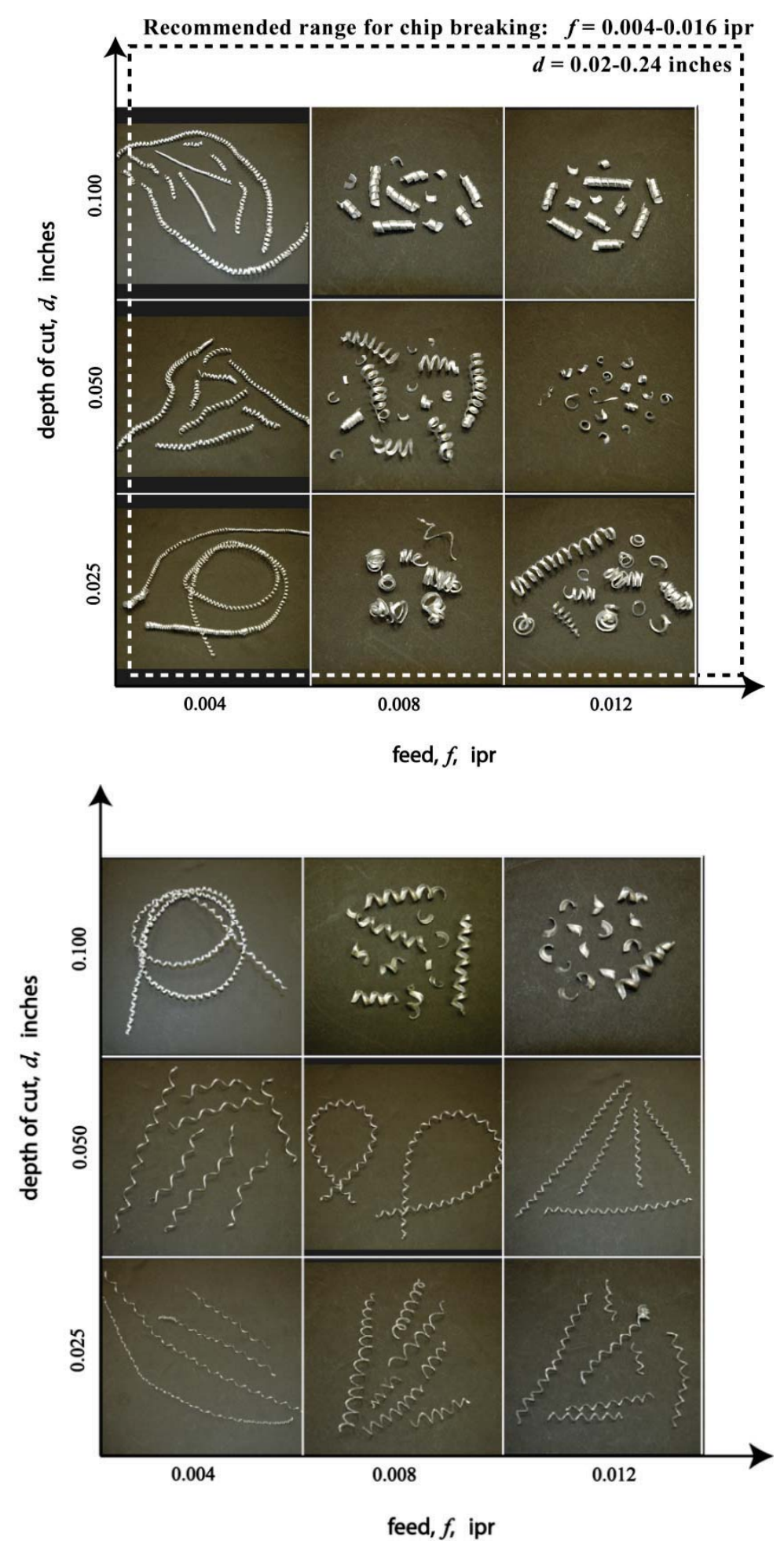

Figure 4.21: Chip morphology for: (top) Korloy SCGT-432 insert (bottom) UCB tool. Work material $=$ AL 2024, $\psi_{r}=15^{\circ}$. 
The observations from the chip charts are noted below:

1. Considering Figures $4.18-4.19$, the AISI 1018 chips produced by the Sandvik insert vary in form depending on the cutting conditions. At $\psi_{r}=45^{\circ}$, the chips forms are for the most part helical with a transition to a snarled form at a combination of large depth and large feed. When the tool lead angle is reduced to $\psi_{r}=15^{0}$, the chip form transitions from helical to small c-shaped segments when the feed/depth cutting conditions change from lower to higher values. Intense oxidation of the chip (deep blue color) are observed at the large depths and large feeds indicating the high cutting temperatures involved (Venkatesh et al., 1991). From the results it can be inferred that the present chip breaker geometry is most effective at lower lead angles using high feed, high depth machining conditions.

2. Chip charts for the AISI 1018 tests using UCB tooling are also shown in Figures 4.18 - 4.19. For the most part, at both $\psi_{r}=45^{\circ}$ as well as $\psi_{r}=15^{0}$, the chip forms are all helical in nature. The helix pitch varies depending on the cutting condition. The chip color, a golden brown at the highest feed and depth combination, for both the lead angles indicate much cooler cutting temperatures - a characteristic of the positive cutting geometries.

Similarly, from the chip charts for AL 2024 shown in Figures 4.20 - 4.21, the following observations are made:

1. Chip forms for the Korloy insert display a broader variation in form. At $\psi_{r}=45^{0}$, the chips are a highly helical form at the lower feeds and transition to a spiral overbroken form at the high feed/depth combination. Note the single long unbroken helical chip 
at $d=0.100 \mathrm{inch}, f=0.008 \mathrm{ipr}$ which is unusual. Replicated experiments consistently resulted in the same chip form at this cutting condition. When the lead angle is changed to $\psi_{r}=15^{0}$, the chips attain forms such as snarled $(d=0.025$ inch, $f=0.008$ ipr), overbroken spirals ( $d=0.050$ inch, $f=0.012 \mathrm{ipr}$ ) and helical.

2. In comparison, the chips for the UCB tool exhibit a consistent helical form at both $\psi_{r}=45^{\circ}$ and $\psi_{r}=15^{0}$. Only the lengths of the chips are different.

\subsection{Concluding Remarks}

This work attempts to understand chip behavior for a model based tool top form geometry. The experimental investigations used both qualitative and quantitative techniques to characterize chip flow as well as chip forms.

Based on the experimental result, the use of Stabler's rule in the model to dictate chip flow was found to be inadequate with large deviations observed between experimental values and the theoretical considerations used in the model. Several different hypotheses for the deviations found in the literature were considered to better explain the inconsistencies in the results. However, none of the hypothesis proposed in the present body of knowledge could adequately explain the conundrum of Stabler's rule. It is concluded that more work needs to be done to quantify the deviations observed in the experimental work on chip side flow. It is envisioned that a thorough investigation into the dynamics within the shear zone for the proposed tool geometry will throw more light on the nature of deviations observed.

Qualitatively, the experiments provide an insight into the flow characteristics and chip forms for the proposed tool geometry. From a comparative study using commercial 
cutting inserts with chip breakers it was shown that present chip breaker geometries are inadequate to effectively address chip control even within the recommended range of cutting parameters. The UCB tool geometry proposed generates consistent chip flow patterns with the chip reaching the pre-determined target as set in the model. However, the consistency was more an outcome of chip side-curl rather than from side flow alone as envisaged in the original model development. Finally, the chip forms are classified via chip charts so as to understand the myriad possible shapes that could result as a consequence of using the proposed tool top form geometry.

The results of the experimental investigations, and in particular, the consistent chip flow pattern observed in the UCB tool, sets the stage for a tool design capable of comprehensively, consistently and universally breaking the chips. 


\section{CHAPTER 5}

\section{A NEW METHOD FOR CHIP BREAKING}

In this chapter, a new method to break chips by bending it backwards is described. Backward bending is achieved by using a novel tooling system design. The tool design is based upon the results of the chip flow for the UCB tool presented in the previous chapter. Results from the cutting tests carried out to evaluate the new tool design concept are presented in this chapter. A case is made for total chip control using the backward bending technique.

\subsection{Introduction}

The problem of total chip control as laid out by Jawahir (1988) and others has not received adequate attention in the field of chip control research. Although much is known about the chip flow and breaking mechanisms, the real challenge in achieving total chip control is actually breaking the chips into consistently acceptable chip forms. The term "acceptable" itself is a fairly subjective description depending upon user opinion. However, standards such as the ISO 3685:1993 provide guidelines on what could constitute acceptable chip forms. Figure 5.1 shows the acceptable chip forms proposed by the ISO 3685:1993 standard. In studying the applicability of the chip form classification in the 


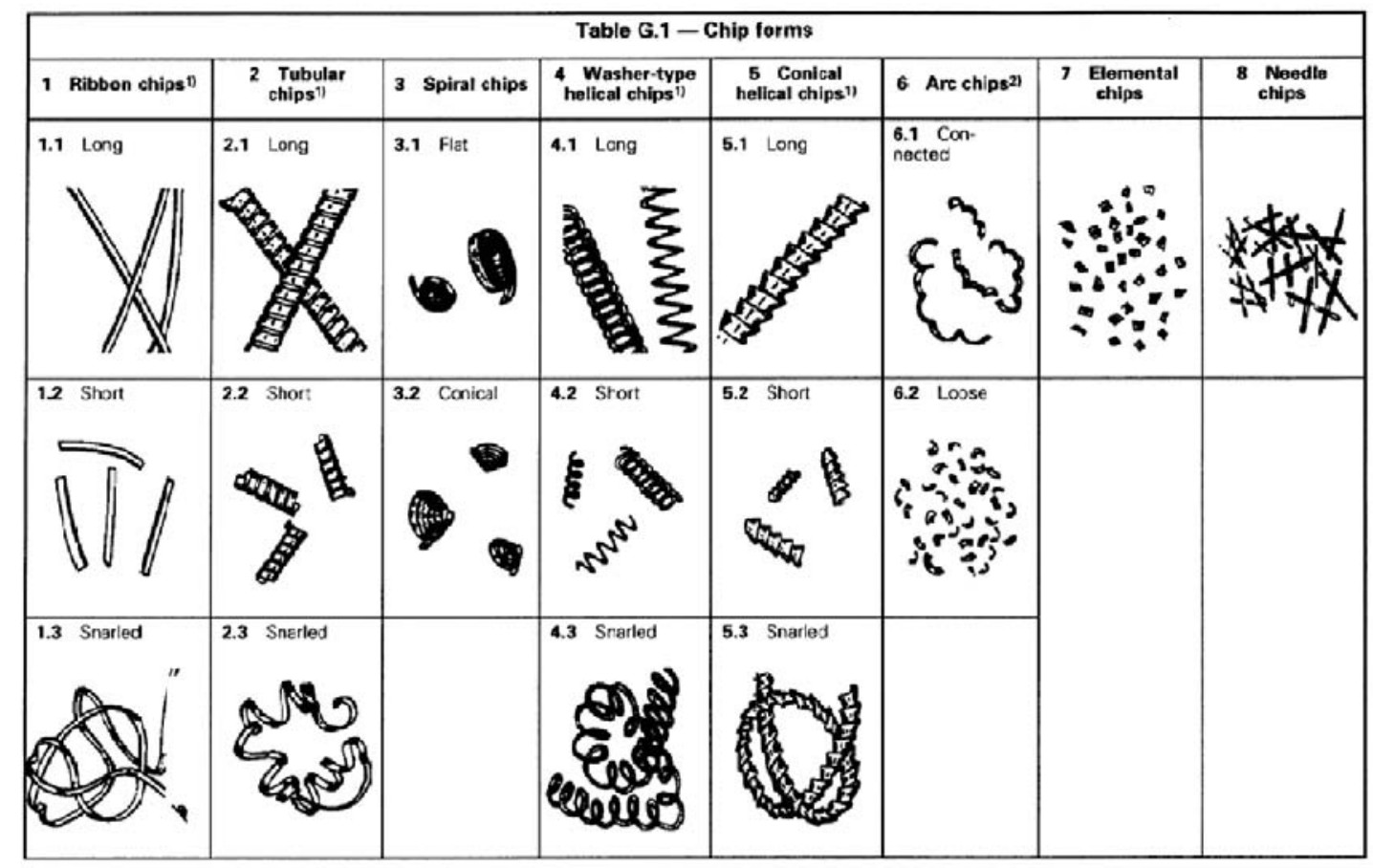

Figure 5.1: Chip form classification based on the ISO3685:1993 standard $^{2}$.

standard, no recommendation or mention is made of acceptable chip forms. However, chip forms of the types \#4.2, \#6.1, \#6.2 and their mixed varieties have been deemed acceptable (Grzesik and Kwiatkowska, 1996). A strong argument can also be made to include chip forms of the types \#1.2 (short ribbon), \#2.2 (short tubular) and \#7 (elemental). Note that the latter inclusions are again subjective and based on other definitions of acceptable chip forms (Shaw, 2005; Zhang, 1995).

\footnotetext{
${ }^{2}$ This material is reproduced from ISO 3685:1993 with the permission of the American National Standards Institute (ANSI) on behalf of the International Organization for Standardization (ISO). No part of this material may be copied or reproduced in any form, electronic retrieval system or otherwise or made available on the internet, a public network, by satellite or otherwise without the prior written consent of the ANSI. Copies of this standard may be purchased from the ANSI, 25 West $43^{\text {rd }}$ Street, New York, NY 10036, (212) 642-4900, http://webstore.ansi.org.
} 
The discussion on acceptable chip forms and their subjectivity serves to highlight the real issue at hand: achieving one or the other deemed acceptable chip forms on a constant basis has so far not been achieved in any of the multitudes of chip breaking techniques currently in use of tried in the past. Towards achieving total chip control, a new tooling system as outlined in the Chapter 3 (see Section 3.1 on Motivations for Universal Chip Control) was developed and tested for its applicability. The concept of backward bending or loading the chip in tension (Nakayama, 1963) is used as a means to effectively break chips into consistently acceptable forms. Very little work, if at all, exists on utilizing backward bending for chip breakage. Nakayama and Arai (1980) have compiled a list of techniques used/tried in the past to achieve total chip control. Their list includes an entry for "winding on a reel" (method) with a "chip guide and reel" (technique) as one way of achieving chip backward bending. But an extended search for a report on such a method resulted in only one related work - a patent for a tool system for guiding a continuous chip formed in turning (Kimura et al., Patent \# 4,271,734, 1981). The cited work uses a complicated design with a rolling spool to induce breakage. Taking cognizance of the complexity of such a system, a more passive approach is used in this research in that no moving parts are used at all. Only the reactionary forces between the chip flow and a static restriction is used for both backward bending as well as breaking of the chip. 


\subsection{Tool System Design for Chip Breaking by Backward Bend- ing}

Many of the design cues for the proposed tooling system are based on an initial rendering of such a concept (EMI, 2005). The design comprises of a two component design - the UCB tool with a geometry providing the direction for chip flow and, an added component to capture and break the chip. While the model-based top form geometry addressed the primary objective of providing the requisite chip flow, the second objective of capturing the chip and subsequently breaking it by backward bending is achieved using an add-on top cover to the UCB tool. The obvious question that arises here relates to the possibility of changing the inherent curvature of a chip that is already formed. The motivating factor for the consideration of the top cover module in the first place can be traced to the work of Kharkevich and Venuvinod (2002), who found that the up-curl of a helical chip could be completely eliminated by the action of an external deformation on the chip flow. On the other hand, the side-curl of the chip was found to be preserved intact. Based on the experimental observations of chip flow and form in Chapter 4, it was hypothesized that the natural up-curl of the chip could be subdued and a forced backward bending initiated on the final chip form by using a suitable top cover design.

Figure 5.2 shows the prototype of the two component tool system. The top cover is machined to match the UCB tool profile and mounted using two screws. A channel machined on the mating surface of the top cover provides additional guidance to the chip flow (by confining it to a finite zone around the target point) and prevents extraneous ef- 


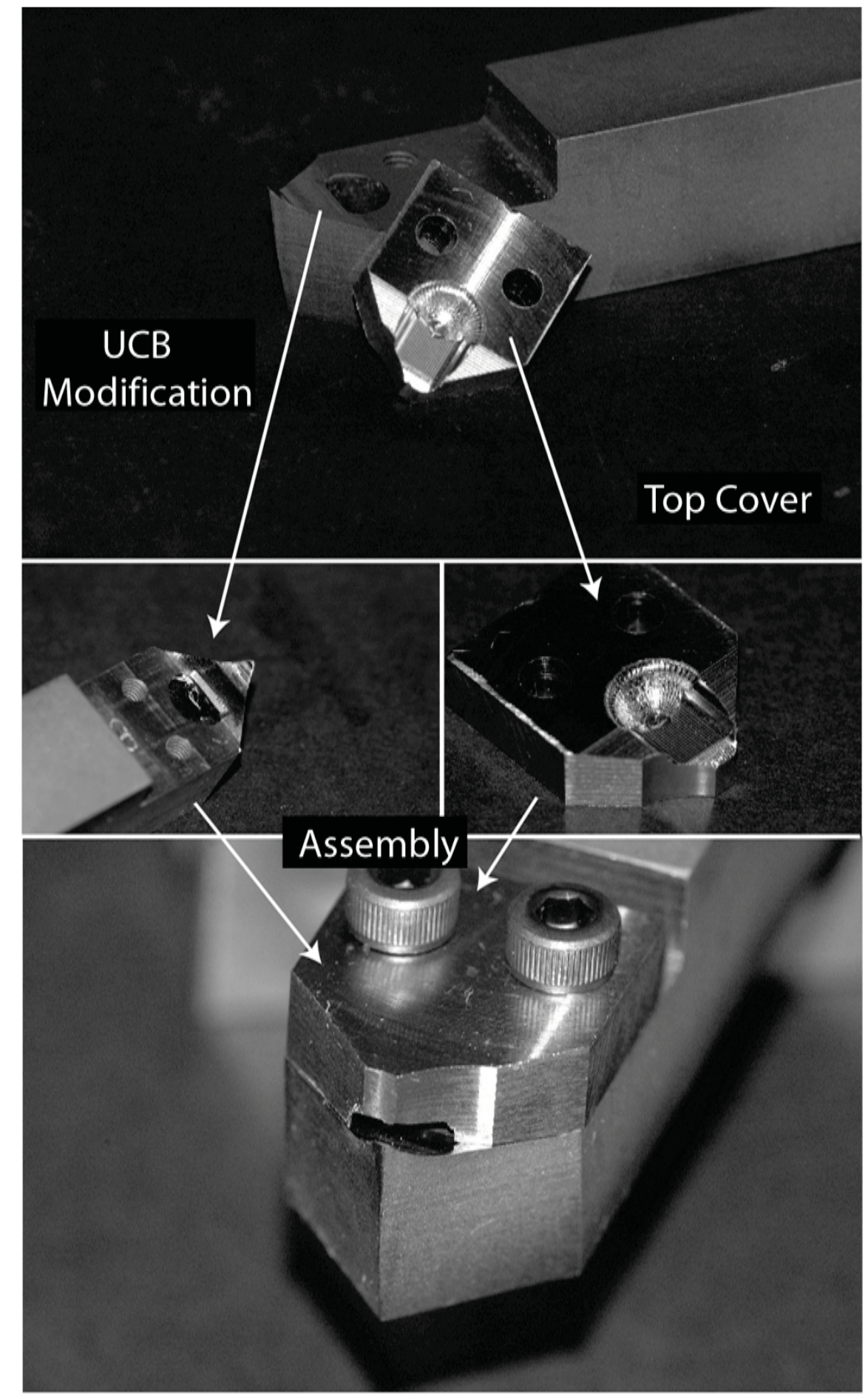

Figure 5.2: Components and design of the tooling system to achieve chip breakage by backward bending. 
fects from altering the flow path of the chip. The channel geometry was designed based on the following considerations (see also APPENDIX F):

1. The cross sectional area is based on measurements of chips collected from the cutting tests of the UCB tool. The height of the channel was set at $4 \mathrm{X}$ the thickest chip for the UCB tool, which was measured at $0.029 \mathrm{inch}$ (AISI 1018 steel, $\psi_{r}=15^{0}$ ).

2. Suitable clearances are provided at the entrance of the channel - on the inner top and side surfaces - to allow the chip to flow unhindered into the channel.

3. At the rear, the channel terminates in a blind wall with a blended spherical bowl design. The spherical bowl (hereon simply called the sphere) provides the necessary backward bending for the free end of the chip.

Similar modifications are also provided to the UCB to accommodate the top cover and facilitate bending. A clearance hole with a fillet radius and wide enough to accommodate the widest chip possible is machined at the target location to evacuate the broken chips. The back wall of the clearance hole is positioned 0.025 " behind the sphere to provide adequate clearance for the chip flow. Additionally, the back wall of the clearance hole also serves as an anchor point for inducing chip breakage. Since the chip would have already started on its backward bending trajectory at the sphere, breakage occurs in the same direction at the clearance hole back wall thus preventing chip clogging. Finally, the restricted contact rake face is machined smooth to provide no restrictions whatsoever for the chip flow. To remove the scallop marks left behind by the ball milling tool, the rake face is hand polished first with a rubber grinding wheel (CRATEX rubberized abrasive wheel, Shape B52 with standard bonded XF grit) followed by a super finishing 
process using an abrasive mounted cotton grinding wheel (CRATEX MX abrasive wheel Shape B52 with CXG bond) in conjunction with a diamond based polishing paste.

Note that chip breakage in the proposed tooling system is achieved either by the action of the spherical bowl, the back wall of the clearance hole or by a combined action of the two features. Based on Nakayama's (1963) results on the mode of loading (compressive or tensile) in chip breaking, the ease of breaking increases with an increase in the curvature of bending. As such, it is presumed that the curvature provided by the sphere almost $90^{\circ}$ from the initial trajectory - and the subsequent contact between the chip and the back wall of the clearance hole is sufficient to induce breakage in the chip.

\subsection{Experimental Evaluations}

\subsubsection{Experimental Details}

The new tooling system developed is evaluated using the bar turning test setup similar to that described in Chapter 4. Two different work materials, AISI 1018 steel and AL 2024, were used in the experiments with each test replicated three (3) times. In the preliminary evaluations, all of the tests were run dry with no coolant. Subsequently, due to reasons discussed later, additional tests were also conducted with the use of a coolant. All tests were run for a cutting time of 10 seconds.

The primary focus of the cutting tests was to achieve total chip control using the top cover in conjunction with the UCB tool geometry predicted by the model. However, noting the growth in including small inclination angles in commercial inserts for cutting long chipping materials (as seen on the Korloy insert used in previous chapter), a variant of 
the UCB tool with a smaller inclination was used for comparative testing. The rationale in using the UCB tool variant was to evaluate whether the extreme edge inclination angle as seen on the UCB tool is truly meaningful from a tool design perspective or, if a small inclination would suffice to provide an initial directional sense to the chip flow with the actual task of guiding the chip to the target accomplished by the top cover. All discussions hereon will describe the UCB variant with the term "UCB-R" (where, R connotes restricted) to differentiate between the two tools. The small inclination used on the UCB$\mathrm{R}$ tool was obtained by including a specific restriction in the original model. For the purpose of this study, the model generated inclination output was limited to half $\left(\sim 19^{0}\right.$ for the case of an S-433 geometry) the value seen on the UCB tool. Other parameters used in the cutting tests were identical to those used in the study of chip flow. The experimental design parameters are listed in Table 5.1.

Note that as a control test, a flat rake faced tool was also included in the tests on AISI 1018. The use of a flat rake faced tool was excluded from the AL 2024 tests due to the problem with edge buildup. Given the consistency observed in the chip flow for the UCB tool for both $\psi_{r}=45^{0}$ and $\psi_{r}=15^{\circ}$, only one level of lead angle, $\psi_{r}=45^{\circ}$, was considered.

\subsubsection{Experimental Results}

The results from the study are analyzed using only qualitative methods. Chips collected from the cutting tests are characterized using a standard chip chart. For the purpose of this study, acceptable forms are identified as those conforming to chip Types \#6.1, \#6.2 and \#7 in the ISO 3685:1993 standards classification shown in Figure 5.1. Note the exclusion of certain other types that could arguably be considered acceptable 
Table 5.1: Variables used in the experimental design for chip breaking tests.

\begin{tabular}{|c|c|}
\hline & AISI 1018 \\
\hline \multirow[t]{3}{*}{ Tools Used } & Flat rake faced $\mathrm{S}-433$ \\
\hline & UCB S-433 \\
\hline & UCB-R S-433 \\
\hline Lead Angle & $\Psi_{r}=45^{0}$ \\
\hline \multirow{3}{*}{$\begin{array}{l}\text { Cutting Parame- } \\
\text { ters }\end{array}$} & Feed (ipr): $\quad 0.005,0.008,0.014$ \\
\hline & Depth of cut (inches): $\quad 0.025,0.080,0.120$ \\
\hline & Cutting Speed (sfm): 110 \\
\hline \multicolumn{2}{|r|}{$\underline{\mathrm{AL}} 2024$} \\
\hline \multirow[t]{2}{*}{ Tools Used } & UCB S-432 \\
\hline & UCB-R S-432 \\
\hline Lead Angle & $\Psi_{r}=45^{0}$ \\
\hline \multirow{3}{*}{$\begin{array}{l}\text { Cutting Parame- } \\
\text { ters }\end{array}$} & Feed (ipr): $\quad 0.004,0.008,0.012$ \\
\hline & Depth of cut (inches): $0.025,0.050,0.100$ \\
\hline & Cutting Speed (sfm): 225 \\
\hline
\end{tabular}

otherwise. Confining the classification to a narrow range builds in a greater robustness to the proposed chip control technique.

Results from the cutting tests on AISI 1018 and AL 2024 are shown in Figure 5.3 and Figure 5.4 respectively. For the present discussions, only results from a single replication 
of the UCB and UCB-R tools are considered. Results for the flat faced tool and all replications are included in APPENDIX G.

From the AISI 1018 test results shown in Figure 5.3 and also comparing directly with the respective chip charts presented in Chapter 3, the effects of the tooling system with the top cover can be observed in the remarkable change in chip forms at the medium/high levels of feed/depth combinations. With the addition of the top cover, the helical chip forms seen when using only the UCB tool without the top cover (as well as those from Sandvik inserts) transform to small discrete elemental varieties of types \#6.2 and \#7 as seen in Figure 5.1. A similar pattern seems to be repeated in the case of chip forms for the UCB-R tool, though chip jamming (as discussed later) occurred at higher depth and feed conditions. Results from the replicated experiments are shown in APPENDIX G. Note that the high depth and feed conditions could not be consistently replicated due to chip jamming at the higher values of feed/depth conditions. For example, in the UCB tool results (AISI 1018) shown in Figure 5.3, while Replication \#1 shows broken chips for all depth, feed cases, the same could not be repeated across all feed, depth conditions in Replications \#2 - \#3 (APPENDIX G) due to chip jamming (as seen at $d=0.120$ inch, $f$ $=0.014 \mathrm{ipr}$ in Figures F.2 - F.3, APPENDIX G). The same result is also seen for the flat tool, the UCB-R tool as well as in the cutting experiments for AL 2024.

For both the tools, the breakability of the chip reduces drastically at low values of feed/depth with the chip reverting to a long unbroken helical form as seen at the $d=$ 0.025 inch, $f=0.005$ ipr cutting condition. The most obvious explanation for this behavior is that, due to the small cross sectional area of the chip, the chip flow changes at the 

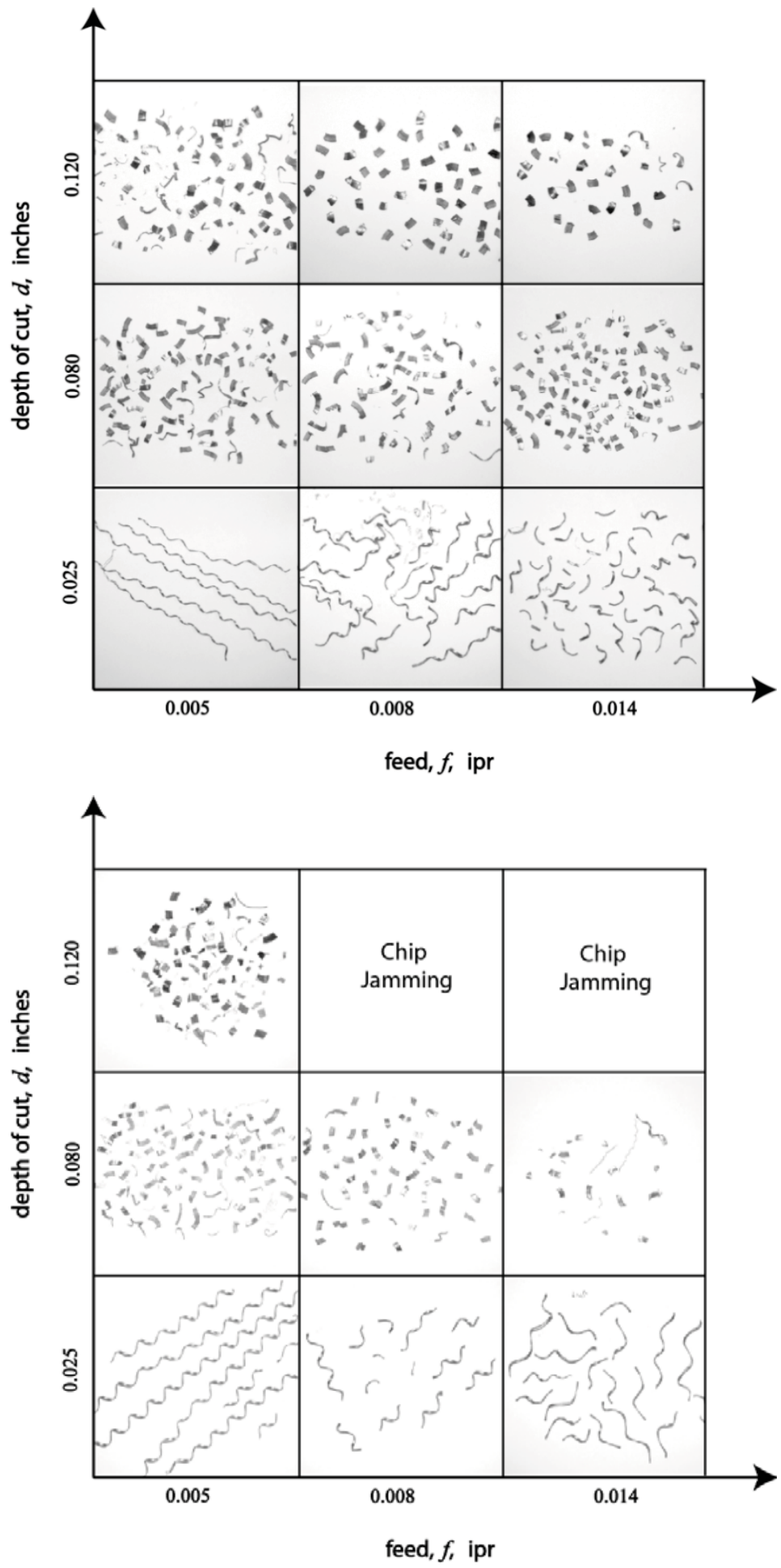

Figure 5.3: Chip charts for: (top) UCB tool (bottom) UCB-R tool; Work material: AISI 1018, $\psi_{r}=\mathbf{4 5}^{\circ}$. 

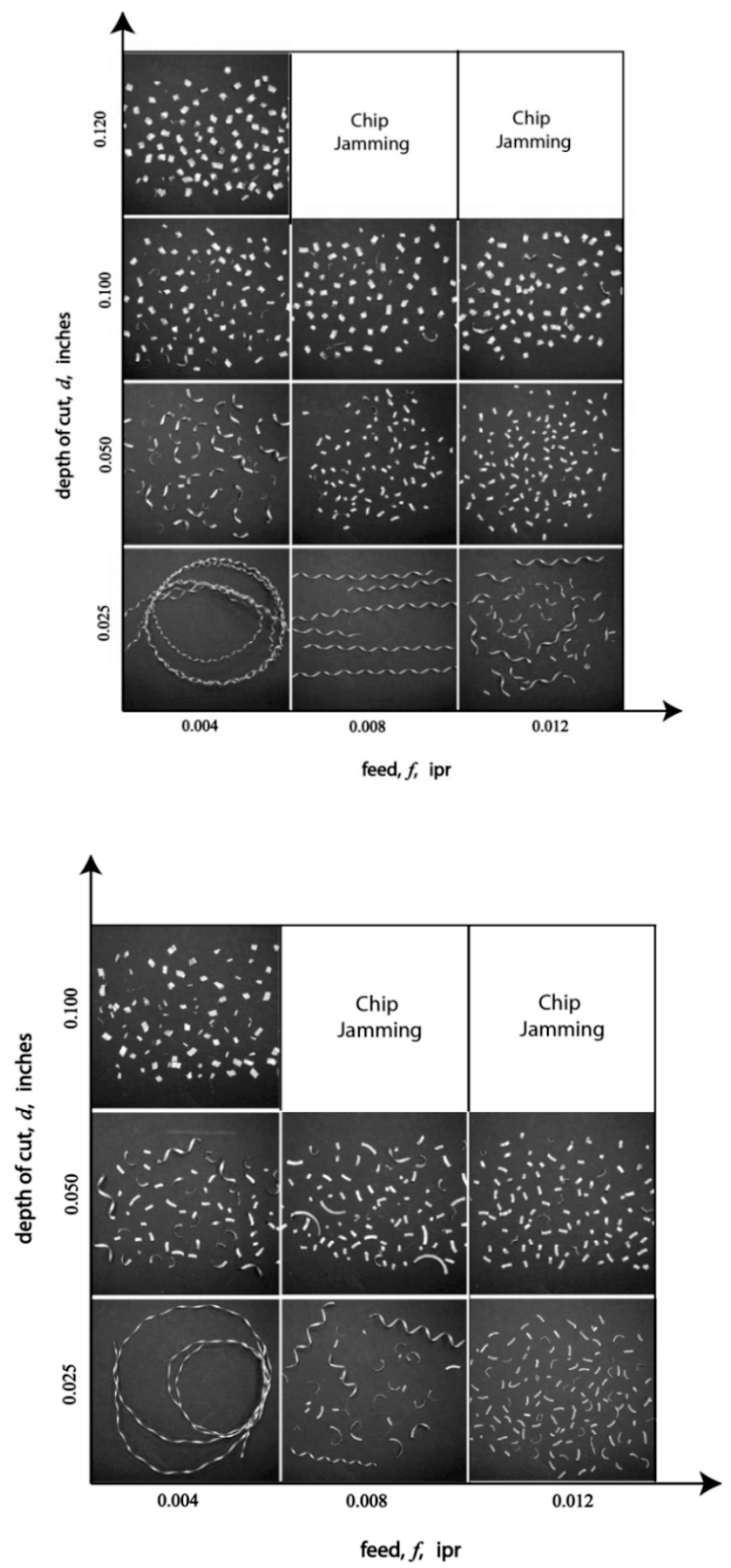

Figure 5.4: Chip charts for: (top) UCB tool (bottom) UCB-R tool; Work material: AL 2024, $\psi_{r}=45^{\circ}$. 
sphere and forms a helix with a diameter much smaller than the cross section of the evacuation hole and hence, the chip does not bend backwards to break at the sphere as is the case with the larger depth/feed case. Rather, the chip seems to flow as a helix (or corkscrew) right through the evacuation hole. Experimental observations indicated this was quite the case as the helical chip twists around its own axis rather than bend when flowing through the clearance hole. The breakability increases with the increase in uncut chip thickness (while still at $d=0.025 \mathrm{inch}$ ). This points to the existence of a limiting uncut chip thickness or alternately, a limiting cross sectional area of the channel geometry to achieve comprehensive breakability. Apart from the breakability factor, the L-shaped form of the broken chip segments confirms the backward bending aspect of the mechanism.

As shown in Figure 5.4, the results from the AL 2024 tests also display an identical trend. Since the chips from the UCB tool showed a reduced propensity to jam, a higher depth condition of $d=0.120$ inches was also included to ascertain the maximum limits of feed/depth combination before chip jamming occurred. At $d=0.120$ inch, chip breaking was successful only at the low feed of $f=0.005 \mathrm{ipr}$, with re-occurrence of jamming at higher feed values.

Given the highly restrictive geometries of the tool design, a further investigation into the exact cause of chip jamming is warranted. From initial observation, edge build up was identified as a strong primary reason for the chip jamming. Despite the highly polished rake face of the tools, a higher buildup was observed when compared to the case of cutting with the top cover removed. Figure 5.5 shows the edge build up from cutting AISI 1018. One possible explanation for the higher build up seen is that the normal force 


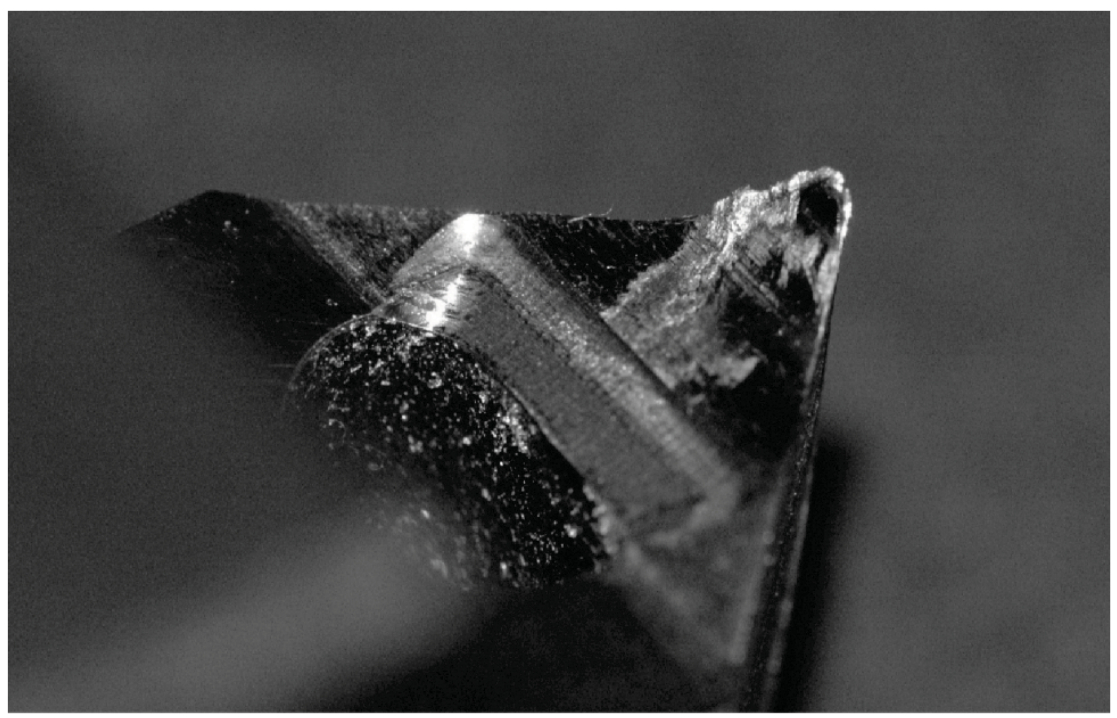

Figure 5.5: Edge build-up from dry cutting using the UCB tool. Work material: AISI 1018, $\psi_{r}=\mathbf{4 5}^{\circ}$.

exerted on the chip surface by the top cover in attenuating the up-curl increases the toolchip contact on the rake face. In any case, edge build up has been reported as a likely cause for a change in chip flow direction (Zorev, 1966). To reduce the edge build up, cutting tests with coolant was subsequently considered. A second factor for the use of coolant was more a result of observation. Trial runs on AL 2024 using a small quantity of aluminum cutting fluid was seen to result in cutting with a lower effort, i.e., the cut "sounded" ${ }^{3 "}$ smoother than dry cutting. Considering that in practice, both AISI 1018 and AL--2024 work materials are machined with considerable coolant application, cutting tests were performed with the application of a water based coolant emulsion (Hocut 795MP semi synthetic). The coolant delivery was standard low pressure flood cooling method.

\footnotetext{
${ }^{3}$ Note that it is common for experienced practitioners (served well by the 8 years of practical industrial experience of the author) to judge machining process conditions using auditory cues from the cutting process. 
Since chip jamming was observed only at the large feed/depth conditions in the initial dry cutting experiments, only two high feed conditions $(f=0.008$ ipr and $f=0.014$ ipr $)$ were used while the depth of cut was maintained at the largest value, i.e. $d=0.120$ inch for both the work materials. No change was observed in the cutting tests for AISI 1018 with chip jamming still a hindrance at the high feed/depth conditions. On the other hand, as seen in Figure 5.6 and Figure 5.7, the chip breaking range for both UCB and UCB-R increased to include the former feed/depth values where jamming was observed. No

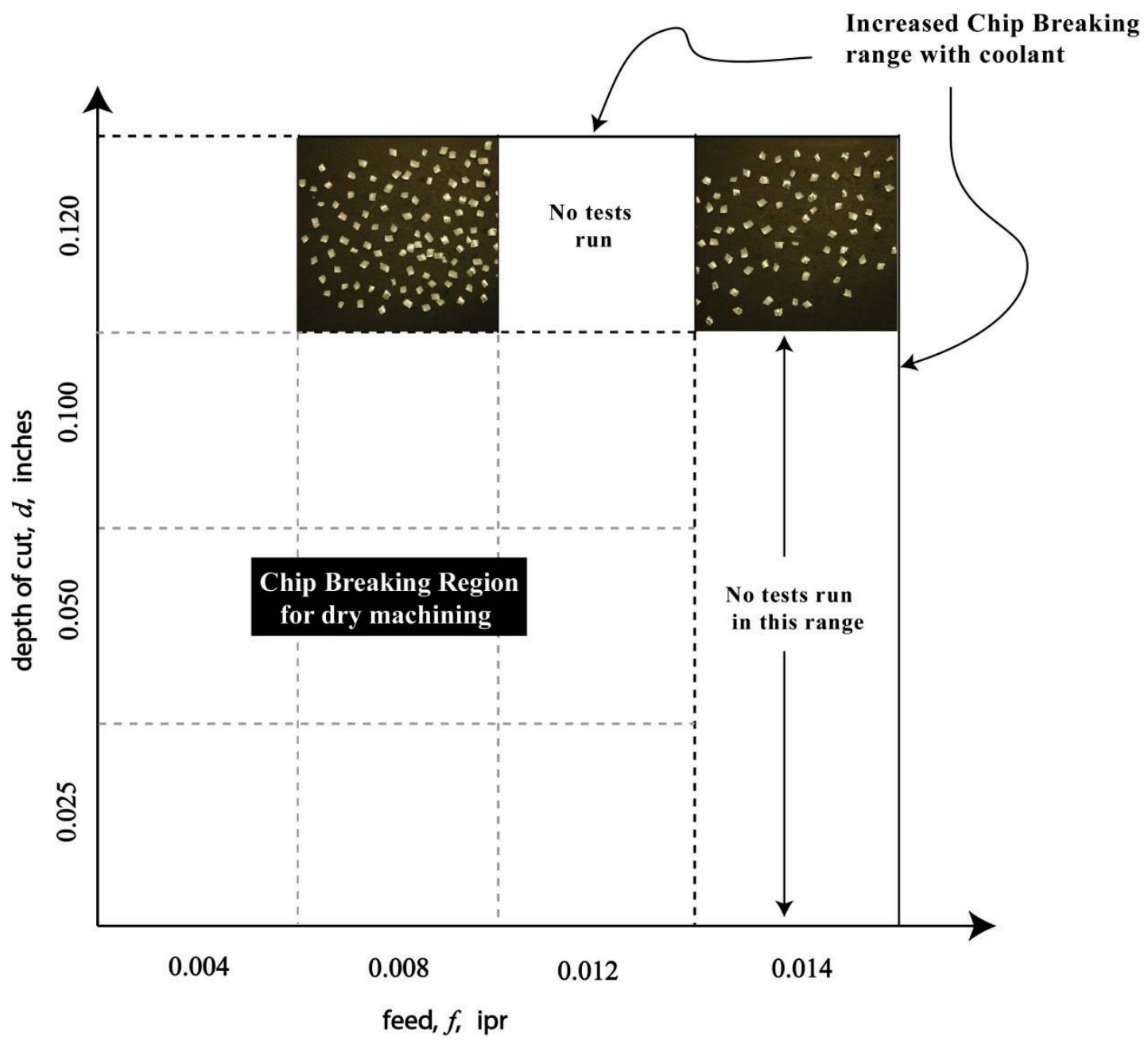

Figure 5.6: Increased chip breaking region due to coolant application in the UCB tool. Work material: AL 2024, $\psi_{r}=4^{\circ}$. 
jamming was observed in any of the three replications performed (APPENDIX H). Further, by using coolant in the cutting process, the edge buildup seen in dry machining reduces drastically with minimal or no buildup observed.

The relatively unchanged results for AISI 1018 when machining with coolant in conjunction with a reduction in edge build up, indicates that edge build up has an insignificant effect on chip jamming. Close scrutiny revealed that the chip jamming was in fact, a result of chip material being forced to wedge between the tool rake face and the top cover due to the chip's thin edge (inner side towards the tool corner radius region)

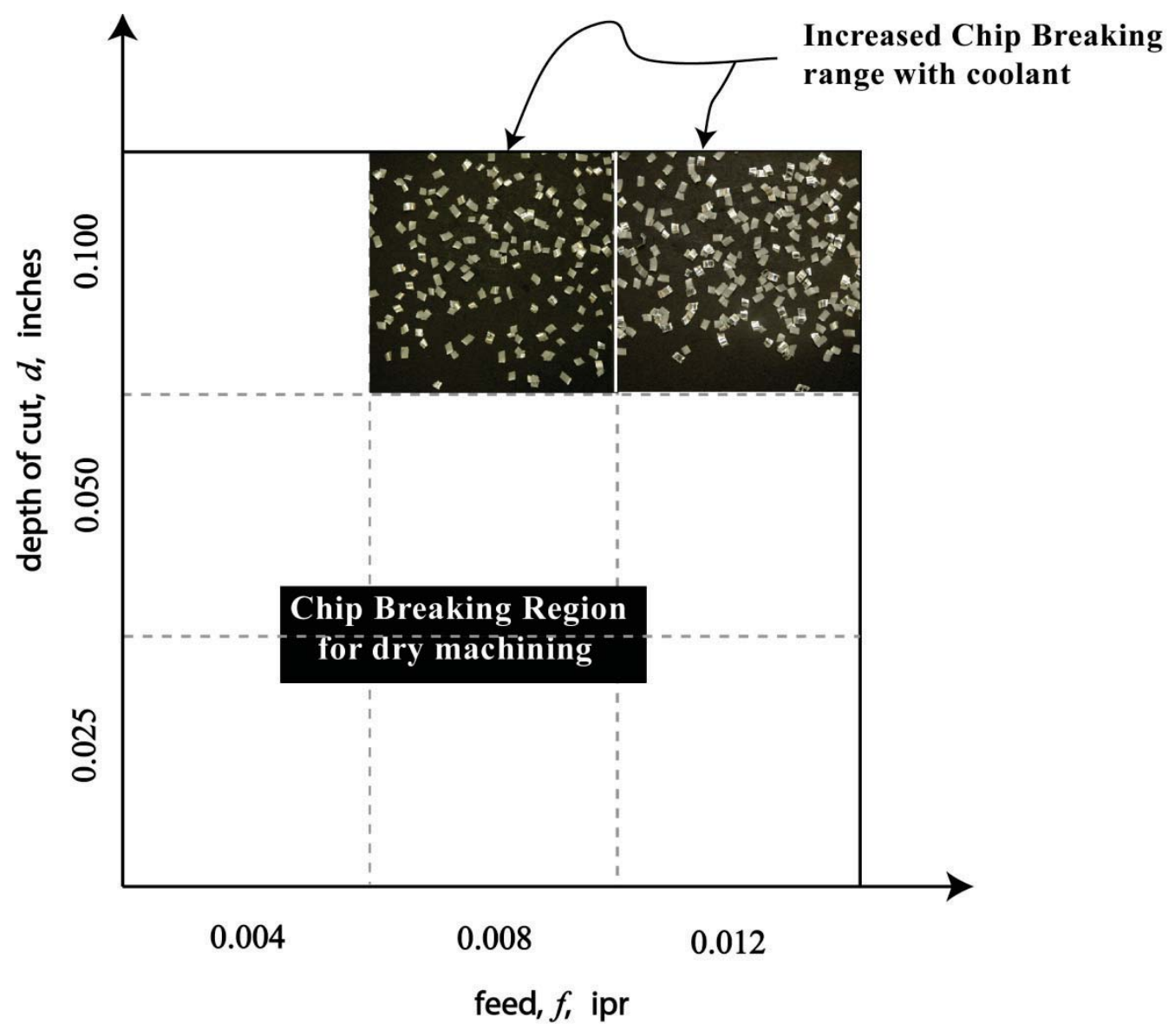

Figure 5.7: Increased chip breaking region due to coolant application in the UCB-R tool. Work material: AL 2024, $\psi_{r}=45^{\circ}$. 
wedging between the tool rake face and the top cover. The sudden restriction in chip flow velocity resulting from the wedging creates a momentary change in flow inertia, leading to subsequent chip material piling up at the point of deceleration. Evidence of the chip-top cover wedging can be seen in Figure 5.8. Note the deformation of the chip edge where wedging occurs. The amount of chip deformation seen points to a high enough pressure that could easily cause the chip material to be forced under the top cover. What is interesting is that chip jamming occurs at higher feed/depth conditions for AL 2024. This is attributed to the fact that steel being stiffer than aluminum, exerts a considerably higher force at the chip-top cover interface resulting in a greater propensity to jam at a lower feed and depth conditions compared to AL 2024.

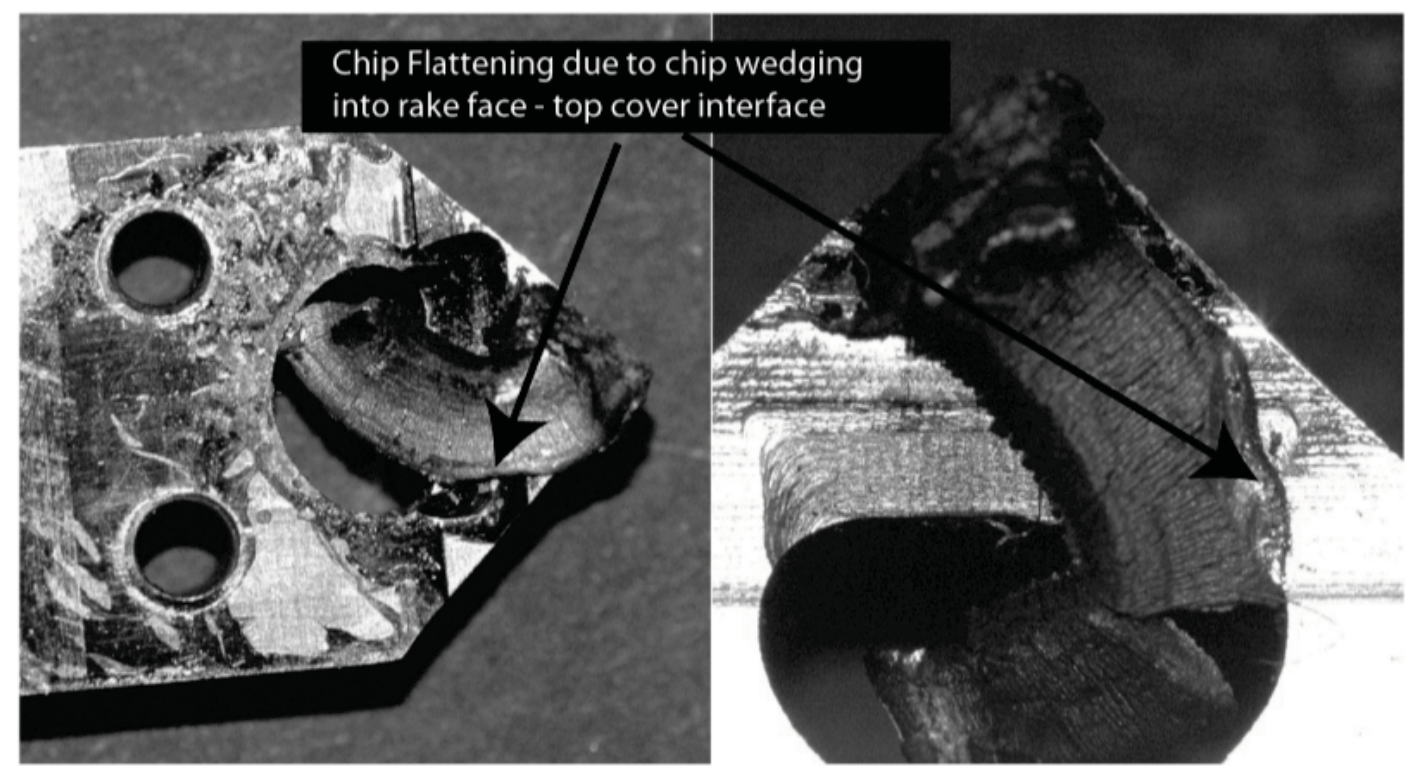

Figure 5.8: Chip flattening as a result of chip wedging between tool rake face and top cover. Work material: AISI 1018, $\psi_{r}=\mathbf{4 5}^{\circ}$. 
The chip-top cover contact mechanism can also be extended to explain the relative decrease in the cutting parameter range for the UCB-R tool. Contextually, the case of the flat rake faced tool (used in AISI 1018 cutting tests only) is also juxtaposed here to help better understand the differences in flow patterns for each tool and the consequent variations in the chip breaking range. Figure 5.9(a) shows the change in the chip breaking range observed for the three tools. The results can be aptly explained by considering the chip flow arrows for each tool as illustrated in Figure 5.9(b). It can be seen that as the maximum inclination angle progressively increases - from $\lambda=0^{0}$ for the flat rake faced tool to $\lambda=19^{0}$ (approx) for the UCB-R tool to $\lambda=38^{0}$ (approx) for the UCB - the chip side flow also increases. It is assumed here, for the sake of illustration, that a linear relationship exists between inclination angle and side flow angle (side flow angle increases with increasing inclination angle) despite the quantitative deviations seen in Stabler's rule. This is a fact corroborated universally by researchers on either side of the Stabler's rule debate. For the case of the UCB with its high inclination angle, the flow direction points closer toward the target location. This is better understood when viewing the direction arrows shown for each tool in the illustration. In the case of the UCB, a direct consequence of the increased side flow angle results in a commensurate decrease in instances of chip jamming, which is evidenced in the larger chip breaking range when compared to the other tools used. In other words, the use of the higher inclination angles on the UCB is justified to achieve effective chip breakage across a wide range of cutting conditions. 


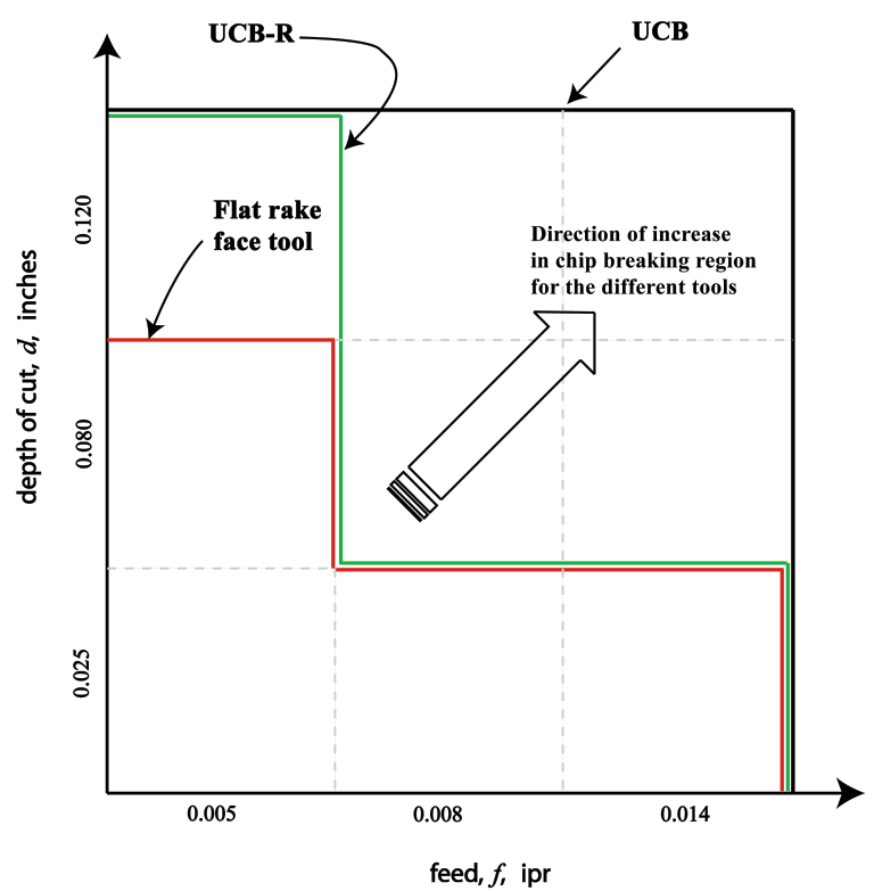

(a)

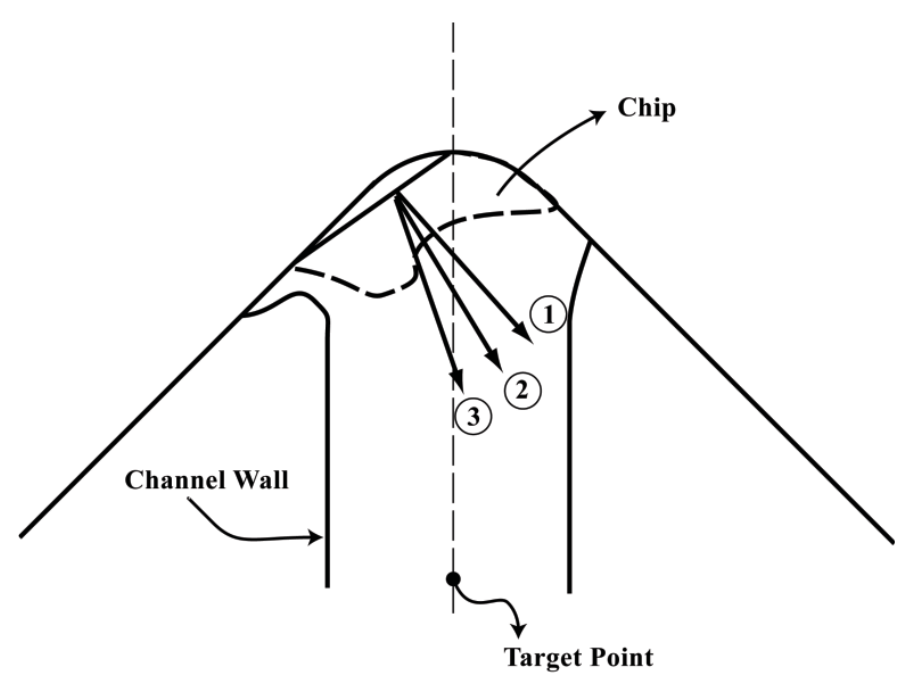

(1) Direction of chip flow in Flat rake faced tool

(2) Direction of chip flow in UCB-R tool

(3) Direction of chip flow in UCB tool

(b)

Figure 5.9: (a) Increase in chip breaking region for different tool geometries (b) Schematic explaining the reason for increase in chip breaking region in different tool geometries. 
Finally, an attempt was made to address the unbroken chip forms at the low range of the feed and depth spectrum. As was mentioned previously, the unbroken chips seen at the low values of cutting parameters is due to the chip twisting rather than bending at the end of the top cover channel. Since the design of the top cover is based on the possibility of eliminating up-curl, the higher clearance of the top cover channel associated with the cut chip thickness at the high depth/feed combination was reduced to $\sim 2 \mathrm{X}$ times the measured chip thickness at the low depth of cut. Replicated tests were again run at the low depth condition, $d=0.025$ inch, with the corresponding three levels of feed. Results of cutting tests using a top cover with lower channel profile are shown in Figure 5.10. As can be seen from the resulting chip images, the lower channel height led to better breakability of the unbroken long helical chip forms previously observed with the higher channel height of the top cover.

Another interesting observation is the apparent shift seen in the chip breaking region

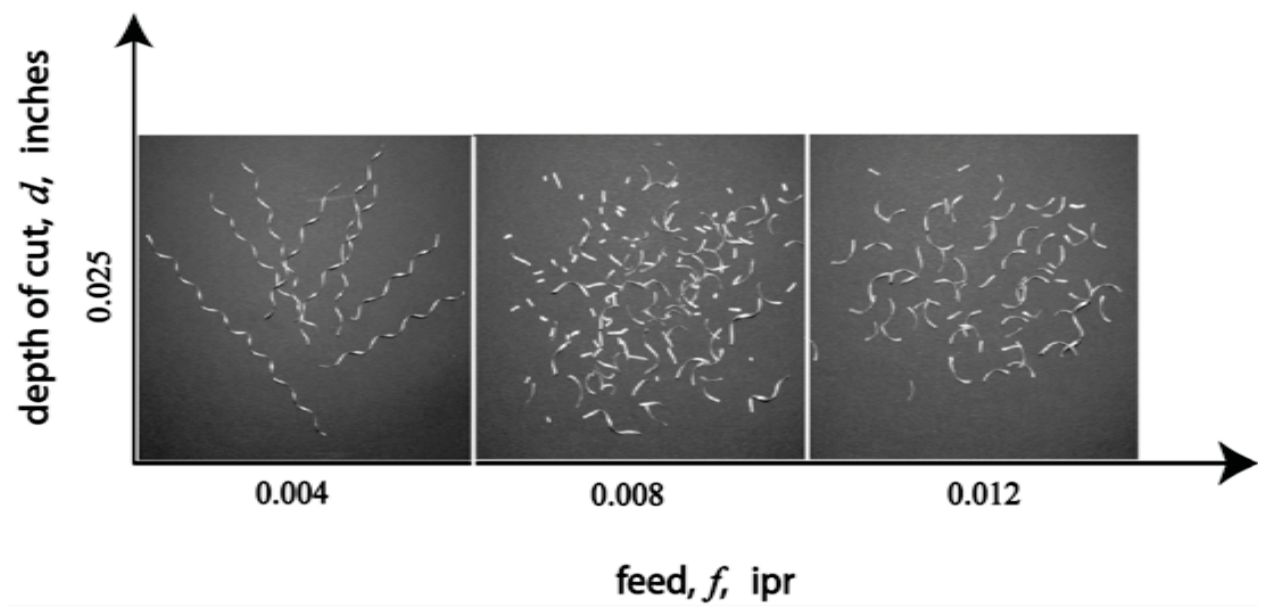

Figure 5.10: Chip forms for lower top cover channel profile;

Work material: AL 2024, $\psi_{r}=4^{\circ}$. 
when compared with the results of the dry cutting tests shown in Figure 5.4. The entire range of feed (for $d=0.025$ inch) appears to be shifted left in the direction of lower feeds. Figure 5.11 shows the shift in the chip breaking region along with the extrapolated chip form one might expect to see at low feeds, i.e., $f<0.004$ ipr. The obvious inference is that there exist critical values for both feed and the top cover geometry below which the chips would remain unbroken. However, recognizing that the issue is more of an optimization of the top cover geometries, it may be concluded that effective chip breakage can be induced even at low feed and depth condition with suitable design changes to the tooling system.

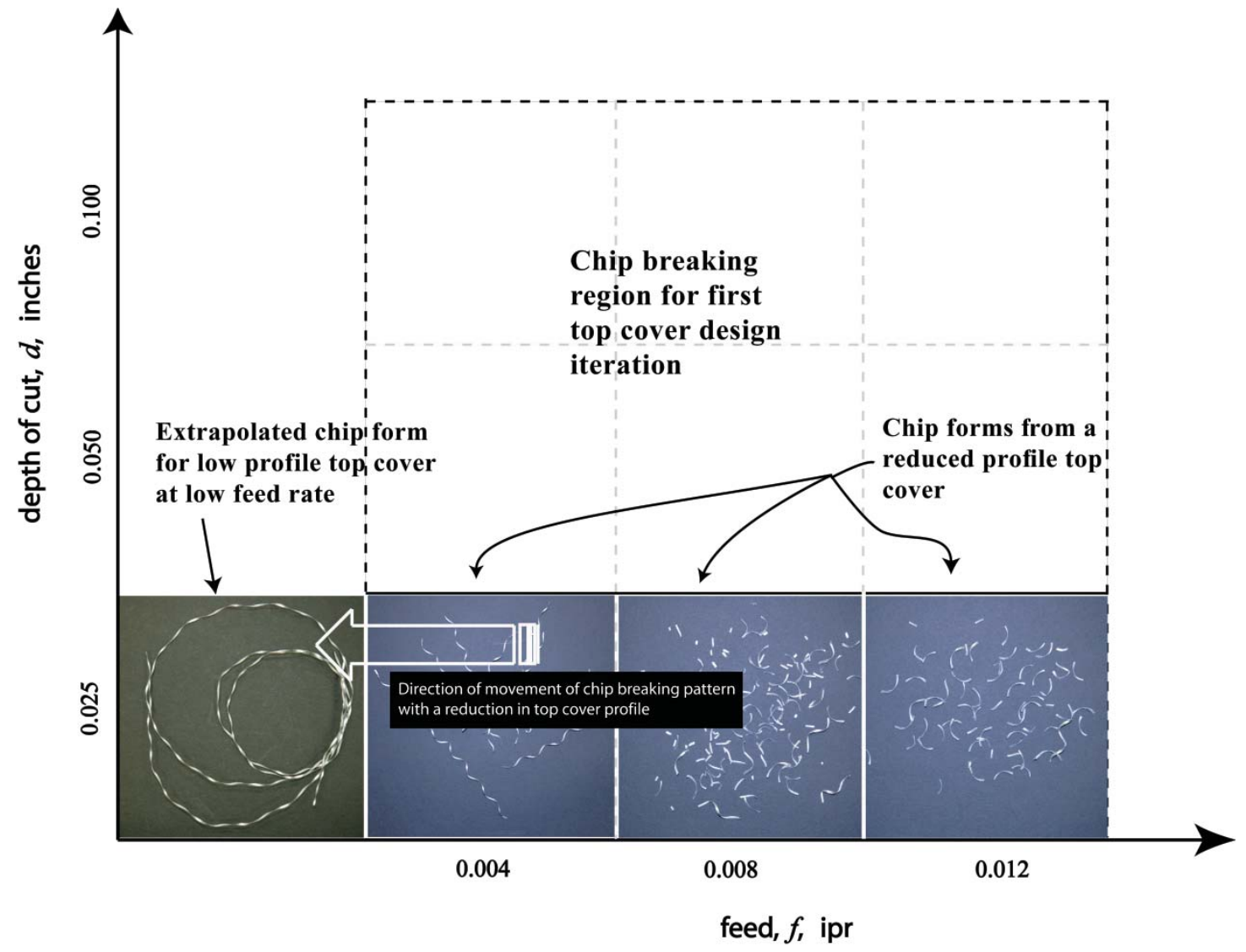

Figure 5.11: Chip chart showing the apparent shift of breaking regions under lower top cover channel; Work material: AL 2024, $\psi_{r}=\mathbf{4 5}^{\circ}$. 


\subsection{Concluding Remarks}

A new tool system design is presented as a means of achieving total chip control in machining. The innovative tooling design achieves chip breaking by backward bending, which so far has not been attempted. Feasibility trials with the proposed design proved that chip breaking by backward bending is possible. From the results, it was shown that chip forms from cutting with the Universal Chip Breaker (UCB) tool exhibit better breakability than existing chip breaker solutions. Several issues concerning the practical application of such a tool were also explored via experimental observations. Some potential reasons inhibiting a broader application of this technique are also identified. 


\section{CHAPTER 6}

\section{CONCLUSIONS AND RECOMMENDATIONS FOR FUTURE WORK}

Significant conclusions from the study on feasibility of a Universal Chip Breaking (UCB) system are presented in this chapter. Several recommendations are made for future work to be accomplished in order to further develop this technique into a robust and viable alternative to the current existing chip breaker technologies.

\subsection{Conclusions and Relevance of the Research}

Based on findings from the two-fold experimental work undertaken - chip flow control and breaking mechanism - the main conclusions are as follows:

Conclusions from chip flow studies

1. Existing chip breaker designs are inadequate to achieve total chip control. The ad hoc designs currently in use greatly limit the use of any single geometry design for achieving comprehensive chip breakage across universal cutting conditions. Based on observations of the chip flow, the geometry of the chip breakers is not fully utilized by the chips even within the recommended cutting range. 
2. Direct use of Stabler's rule in the modeling effort is found inadequate. Stabler's rule was the driving assumption in the model used for the development of a variable tool edge inclination angle to engineer chip flow to a predetermined target location. It was presumed that Stabler's rule would be sufficiently satisfied since it is applied on only small elemental edge segments. Experimental results, however, show considerable deviations from the theoretical values.

3. Deviations of Stabler's rule, seen even under minimal tool chip contact conditions of the restricted rake face geometry, contradicts the common notion of frictional effects being the cause of the deviations. Clearly, factors other than pure geometry and friction are at work to cause the deviation seen in the experimental results.

4. Even though the straight side flow of the chip was the primary control objective to guide the chip to the target location, it is seen that the chip flow pattern for the UCB tool geometry is dominated by the side-curl rather than the straight side flow. While the combined effects of side flow and side-curl enabled the chip to reach the designated target point, it is observed that the result is more due to side-curl of the chip rather than straight flow. Moreover, due the predominant side-curl of the chip flow, the chip end exhibits an orientation of the chip width that was not anticipated.

5. Based on the chip flow pattern observed with the UCB tool geometry, the location of the target is critical. For the chip to consistently reach the target, the target location needs to be positioned as close to the cutting edge as possible.

6. Compared to the current chip breaker geometries, the UCB tool geometry exhibits a consistency of chip flow pattern as well as chip form. This consistency is maintained 
across multiple levels of work materials and cutting conditions, particularly the feed and depth of cut.

Conclusions from the investigations on chip breaking:

1. Chip breaking by backward bending, a technique so far unconsidered in chip breaking research, is a viable means to effectively break chips.

2. As opposed to conventional chip breakers, where a large variation in chip form is observed, the design of the universal chip breaker tooling system consistently breaks the chips into the highly desirable form of small segmented units that can be easily evacuated from the cutting zone.

3. A single universal geometry for the chip guidance design to accommodate an entire range of chip cross sectional areas may not be achievable. Multiple chip guidance geometries may be needed to cater to specific cutting parameter ranges, in particular, for very small depths and feed conditions seen in finish machining conditions. Even so, the only changing element in the tooling system is the low wear top cover and not the high wear insert itself.

4. Use of coolants can substantially improve the performance of the UCB tooling system.

In its contribution to the overall chip control problem, several significant impacts can be identified from this research. They are:

1. It is proved that the concept of backward bending for chip breaking can be applied successfully. As it stands, till date, no recorded work was found to exist on the use of such a technique to break chips. 
2. Using the proposed UCB tooling system, the current iterative method of achieving "acceptable" chip forms can be eliminated and consistent segmented chips can be achieved. This presents a chip control solution which is both robust and at the same time simple to implement and use for the general practitioner.

3. The UCB tooling system significantly reduces the number of chip breaker geometries in use today to only three possible variants; only specific top cover designs need be considered for each of the finish, medium and rough machining conditions with no change in the tool top face geometry. In fact, with suitable design refinements, it may be possible to narrow this down to only two top cover variants - for finish and medium-heavy conditions.

The enormity of the last finding can only be grasped by the following fact: from a cursory count of the number of chip breaker variants for only the S-type turning insert geometry in the most recent tooling catalog of a single vendor (Sandvik, 2009a), a total of 13 chip breaker variants exist for specific levels of work material (P, M, N types only), tool configurations (positive, negative geometries) and cutting conditions (rough, medium, finish machining). By reducing the 13 tool variants to only 2-3 variants of the low wear top cover element effectively reduces the 13 tool variants to 1 choice for the tool geometry.

\subsection{Recommendations for Future Work}

The work presented in this dissertation lays the foundations for addressing the issue of total chip control using the novel and seemingly, so far unexplored technique of chip backward bending. Given the strong exploratory nature of this research, many comple- 
menting areas of machining need to be further investigated for their impact on the idea presented in this dissertation. However, to address some of the immediate gaps, a few recommendations are proposed herein.

\subsubsection{Modeling Efforts}

At a fundamental level, a need exists to develop robust models to better predict chip flow for the UCB geometry. The chip side-curl factor also needs to be included into the predictive model to generate a tool geometry capable of directing the chip to the target location. Such a task is by no means trivial. Several variables such as workpiece diameter (Okoshi and Kawata, 1967), tool chip tribology (Balaji, 2000) as well as the complex geometry of the UCB itself will need due consideration. In all probability, the use of em-

pirical work appears to be the best approach for including the effect of the side-curl on chip flow.

More importantly, the problem of inconsistencies seen in Stabler's rule still needs answers. Since the experimental work eliminated the effect of friction as a cause, mor work is needed in understanding the shear zone behavior. Slip-line or finite element models should help in this regard. Even though not considered in this research, a logical next step would be to also model the cutting forces and their orientation to grasp the intricacies of the chip flow in cutting with the UCB.

\subsubsection{Further Development of the UCB Tooling System}

During the course of this research, the tooling system was identified as one of the main areas in need of further supporting work. 
The size of the UCB tool and its effect on chip flow needs a thorough investigation. In this research, due to the convenience associated with the size and shape, the S-433 insert was selected for the cutting tests. For the 0.5 " (inscribed circle) insert size selected, the target point ends up being relatively close to the cutting edge and allows for the chip to reach the chosen target location. From the results presented in Chapter 3, it is also noted that the chip side-curl starts instantly after leaving the cutting edge with only a minimal straight flow. The singular question that arises from the results pertains to the effect of using a large $i C$ size such as a 0.75 " (S644 insert type) or, in extreme cases, a 1" sized insert. The larger insert size along with the sharp chip side-curl observed could result in the chip flow completely missing the target location or, for that matter, not even reaching the vicinity of the target location. As such, further cutting tests are needed to study this aspect if the UCB tooling is to address the range of rough machining conditions.

Another aspect that deserves attention is the extreme tool top face geometries observed as the tool geometry changes from an S-type to a V-type insert. The highly restricted geometry of the $\mathrm{V}$-type insert results in predicted edge inclinations on the order of $55^{0}$ or greater. To highlight this extreme condition, the model predicted UCB form for a V-type insert is shown in Figure 6.1. Note that the inclination angle for the tool shown in the image is restricted to $50^{\circ}$. In preliminary cutting tests, the extreme inclination of the V-type geometry as a UCB tool showed no signs of failure. However, the cutting conditions used were in the range of finish machine conditions. It then becomes necessary that cutting tests be performed to evaluate whether such geometries are practically 


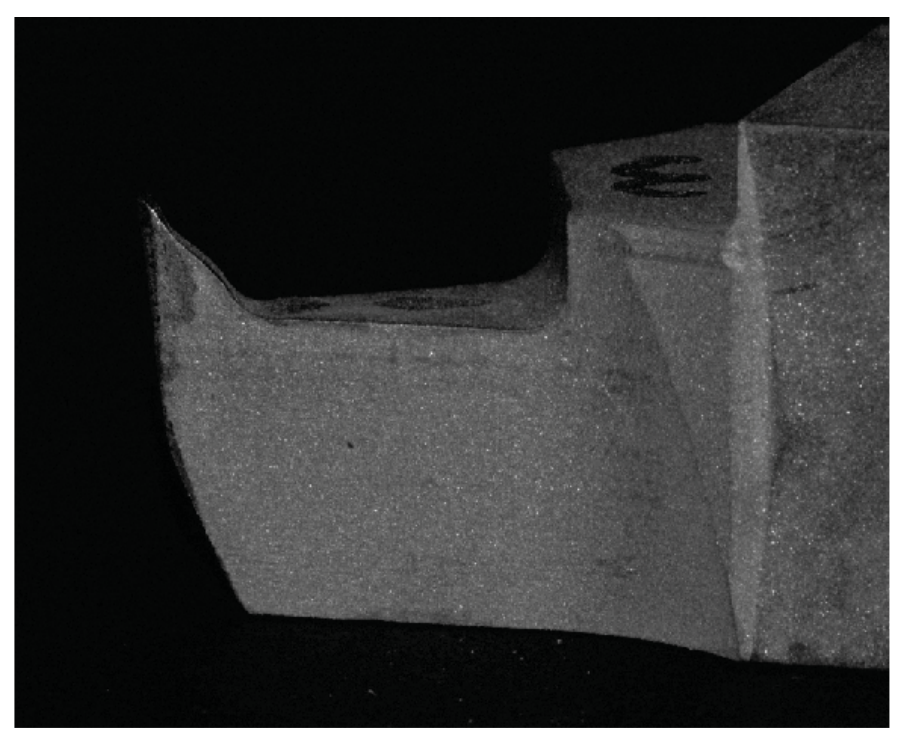

Figure 6.1: Extreme inclinations angle for a V-type insert geometry.

admissible and if so, identifying the range over which such geometries can be used without tool edge failure.

Yet another recommendation on refining the tooling system relates to the development of a true carbide insert with the UCB geometry. Due to the early stage exploratory nature of this work, the UCB tooling system prototype developed was rudimentary and fabricated using A-2 tool steel. The higher toughness of the tool steel ensured no tool failures. However, the low Transverse Rupture Strength (TRS) of the carbide material (Exner and Gurland, 1970) poses formidable challenges in using the UCB geometry in carbide form. It is certainly acknowledged that developing a disposable carbide insert with the UCB geometry is more of a materials issue rather than a pure cutting problem but, given the strong practical implications of the UCB technique, it is nevertheless considered within the scope of future developments. While on the matter of carbide tooling 
development, an interesting aspect of study would definitely be the effect of coatings on the chip flow behavior.

The final note in this section deals with the design of guidance geometries. The present insert guidance system geometry needs further optimization to cater to the two specific issues identified: the cases of chip jamming at large chip cross sections and the unbreakability seen at low feeds. One recommended piece of work is to redesign the top cover so that the seating surface is recessed below the tool top rake face level to prevent the chip from wedging between the two surfaces. This was not accomplished in this work due to insufficient tools available on hand.

\subsubsection{Profile Machining}

A relatively overlooked aspect of the chip control problem is the influence of the tool path on chip control. As with a majority of the research on chip control, the work undertaken in this research pertains only to the case of longitudinal cutting. In reality, machining conditions routinely include both longitudinal cutting as well as profile machining. Profile machining calls for different cutting strategies based on the accessibility of the workpiece geometry (Sandvik, 2010). Further, the effective feed and cutting depth conditions in profile machining constantly change at different points along the contour (Balaji and Jawahir, 2001), presenting a special case of the chip control problem. More recently, Hagiwara et al. (2009) presented a mechanistic model for predicting the changes in instantaneous side flow angle in contour turning of aluminum.

Using a profile geometry similar to that proposed by Balaji and Jawahir (2001), preliminary experiments to study chip flow patterns in commercial inserts (Tungalloy V- 
333, uncoated) and the UCB tool resulted in minimal differences in chip flow between the two tools. No effort was made to machine with a chip guidance/breakage mechanism on the V-type UCB tool. Early indications from the profile machining tests pointedly suggested that the UCB tool may not have an application in certain machining conditions such as profile machining.

Recent developments, however, have indicated that the problem of contour turning is seemingly more complex than is apparent. In a discussion with a major commercial insert vendor ${ }^{4}$, it was indicated that the real crux of chip control in profile machining as seen in the industry is the sudden change in profile seen in machining of corners in large diameter parts. A schematic illustration of such a geometry is shown in Figure 6.2. As such, the exploratory profile tests conducted at small work diameters ( $\phi 2.5 ")$ may have been insufficient to make any conclusions. Based on the new insights gleaned on profile

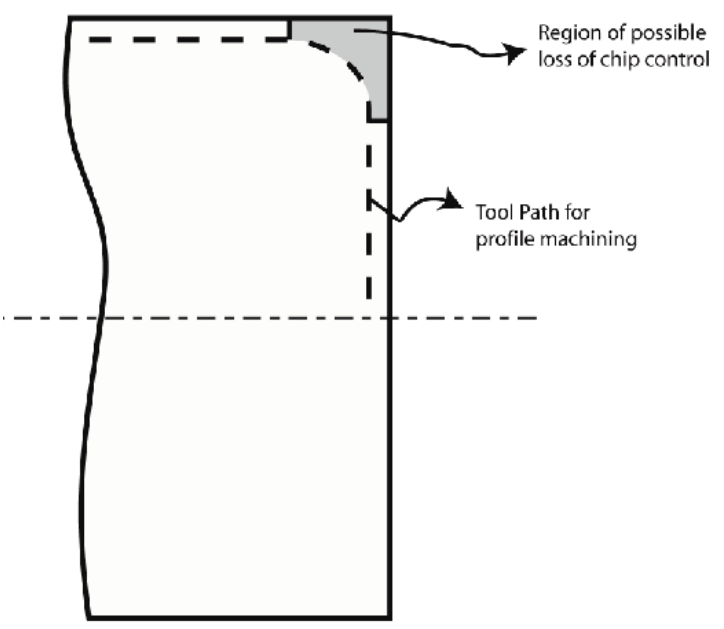

Figure 6.2: Schematic of the profile with a potential for possible loss of chip control.

\footnotetext{
${ }^{4}$ Based on a technical discussion on this matter between Dr. William Endres of EMI and representatives of the Research Division of SECO Tools AB.
} 
machining issues from an industry perspective, further testing is required to study the chip control problem at large diameter start-of-profile machining. It is also required that the complete UCB tooling system with the chip guidance design be used to study the chip breakability under the profile machining conditions mentioned.

\subsubsection{Effect of Cutting Speed}

All the experiments in this research were conducted at very low values of cutting speeds. The low cutting speeds were required since the UCB tool was fabricated out of A-2 tool steel. However, normal cutting speeds for the commonly used carbide inserts are usually substantially higher than those used in the UCB experiments. Some debate exists on the effects of cutting speed on the chip side flow angle. Zorev (1966) presents experimental results showing that the cutting speed has no significant effect on the side flow angle, while other works such as that of Pal and Koenigsberger (1968) indicate that the side flow angle decreases with an increase in cutting speed. If the latter were in fact true, even a marginal straightening of chip flow would result in chip jamming or in the case of wet cutting, reduce the overall range of application. The other potential problem associated with a higher cutting speed is the evacuation of chips from the guidance system unit. A higher cutting speed naturally increases the volume of chip flowing through the top cover unit and as a consequence, chip jamming may result if the breakage and evacuation does not progress at a matching rate. Only a high-speed cutting study with the UCB tooling system - made with carbide - will clarify the exact implications. 


\section{BIBLIOGRAPHY}

Albrecht, P., 1960. "New Developments in the Theory of the Metal Cutting Process. Part 1: The Ploughing Process in Metal Cutting", Journal of Engineering for Industry, V82, pp. 348-357.

Allen, D. K., 1969. Metallurgy Theory and Practice, American Technical Publishers, Inc.

ANSI B212.20 (R-2002), 1980. Carbide Chip Breakers Used with Indexable Inserts for Clamp Type Holders.

Arai, M., 2001. "Study on Chip Control in Metal Machining - On the Sideward Curl of Chip", Journal of the Japanese Society of Precision Engineering, V67, n7, pp. 11641168.

Arrazola, P. J., Ugarte, D., Montoya, J., Villar, A. and Marya, S., 2005. "Finite Element Modeling of Chip Formation Process with Abaqus/Explicit 6.3", $8^{\text {th }}$ International Conference on Computational Plasticity, Barcelona, Spain.

Arsecularatne, J. A., Mathew, P. and Oxley, P. L. B., 1994. "Prediction of Chip Flow Direction and Cutting Forces in Oblique Machining with Nose Radius Tools", Proceedings of the Institution of Mechanical Engineers, V209, pp. 305- 315.

Astakhov, V. P., 2005. "On the Inadequacy of the Single-Shear Plane Model of Chip Formation”, International Journal of Mechanical Sciences, V47, pp. 1649-1672.

Astakhov, V. P., 1999. Metal Cutting Mechanics, CRC Press.

Balaji, A. K., Ghosh, R., Fang, X. D., Stevenson, R. and Jawahir, I. S., 2006. "Performance Based Predictive Models and Optimization Methods for Turning and Applications: Part 2 - Assessment of Chip Forms/ Chip Breakability", Journal of Manufacturing Processes, V8, n2, pp. 144-158.

Balaji, A. K. and Jawahir, I. S., 2003. "Variable Tool-Chip Contact and Chip Morphology in Machining with Grooved Cutting Tools - A Tribological Perspective", Proceedings: Tribology in Environmental Design (TED 2003), Bournemouth, UK, pp. 311-323. 
Balaji, A. K. and Jawahir, I. S., 2001. "A Machining Performance Study in Dry Contour Turning of Aluminum Alloys with Flat Faced and Grooved Diamond Tools", $M a-$ chining Science and Technology, V5, n2, pp. 269-289.

Balaji, A. K., 2000. An Experimental and Theoretical Study of Variable Tool Chip Interfacial Friction and Cyclic Chip Formation in Machining with Grooved Tools, Ph.D. Dissertation, University of Kentucky-Lexington.

Batzer, S. A. and Sutherland, J. W., 2001. "A Geometric Analysis of Semi-Spiral Chip Morphology in Orthogonal Machining", Machining Science and Technology, V5, n1, pp. 63-76.

Batzer, S. A., Sutherland, J. W., and Olson, W. W., 1997. "Chip Morphology and Bending Moment Models for Orthogonal Machining with Flat Faced Tools", Transactions of NAMRI/SME, V25, pp. 231-236.

Bhaktavachalam, B. and Venu Vinod, P. K., 1973. "Side-curl of Chips - Its Importance and Prediction in Metal Cutting", Proceedings of the $6^{\text {th }}$ All Indian Machine Tool Design Research Conference, Bangalore, India, pp. 235-242.

Boothroyd, G. and Knight, W. A., 2006. Fundamentals of Machining and Machine Tools, $3^{\text {rd }}$ Edition, CRC Press.

Brown, R. H. and Armarego, E. J. A ., 1964. "Oblique Machining with Single Cutting Edge”, International Journal of Machine Tool Design and Research, V4, n 1, pp. 924.

Chao, B. T. and Trigger, K. J., 1959. "Controlled Contact Cutting Tools", Transactions of the ASME: Journal of Engineering for Industry, V81, pp. 139-151.

Christopher, J. D., 1990. "The Influence of High Pressure Cryogenic Coolant on Tool Life and Productivity in Turning”, SME Technical Paper MR90-249.

Colwell, L. V., 1954. "Predicting the Angle of Chip Flow for Single Point Cutting Tools", Transactions of the ASME: Journal of Engineering for Industry, V76, n2, pp. 199-204.

Connolly, R. and Rubenstein, C., 1968. "The Mechanics of Continuous Chip Formation in Orthogonal Cutting", International Journal of Machine Tool Design and Research, V8, pp.159-187.

Cook, N. H., Finnie, I. and Shaw, M. C., 1954. "Discontinuous Chip Formation", Transactions of ASME: Journal of Engineering for Industry, V76, pp. 153-162. 
Cook, N. H. and Shaw, M. C., 1951. "Visual Metal Cutting Study", Mechanical Engineering, V73, n11, pp. 922-923.

Danielyan, A. M. and Gritsaenko, Y. A., 1962. "Vibratory Cutting", Machines and Tooling, V33, n6, pp.. 51-52.

DeChiffre, L., 1992. Metal Cutting Mechanics and Applications, D.Sc. Thesis, Technical University of Denmark.

Destefani, J., 2002. "Cutting Tools 101: Geometries", Manufacturing Engineering Magazine, V129, n5.

Dewhurst, P., 1978. "On the Non-Uniqueness of the Machining Process", Proceedings of the Royal Society of London. Series A: Mathematical and Physical Sciences, V360, n1703, pp. 587-610.

Dixit, P. M. and Dixit, U. S., 2008. Modeling of Metal Forming and Machining Processes: by Finite Element and Soft Computing Methods, Springer-Verlag London.

Eastman, M., 1999. "Inserts Show their True Colors", Cutting Tool Engineering, Magazine Issue: April 1999.

Ee, K. C., Balaji, A. K. and Jawahir, I. S., 2003. "Progressive Tool-Wear Mechanisms and their Effects on Chip Curl/Chip-form in Machining with Grooved Tools: An Extended Application of the Equivalent Toolface (ET) Model”, Wear, V255, pp. 14041413.

Endres Machining Innovations LLC, 2005. Internal Report (classified).

Endres, W. J., 2005. Machining Processes: Model Based Planning and Diagnostics, Endres Machining Innovations, LLC.

Endres, W. J., DeVor, R. E. and Kapoor, S. G., 1993. “A Dual Mechanism Approach to the Prediction of Machining Forces: Part 1 - Model Development", Journal of Engineering for Industry, V117, n4, pp. 526-533.

Ernst, H. and Merchant, M.E., 1941. "Chip Formation, Friction and High Quality Machined Surfaces", Transactions of The ASME, V29, pp. 299-378.

Ernst, H., 1938. "Physics of Metal Cutting”, ASM paper on Machining of Metals, Clevelend, $\mathrm{OH}$. 
Exner, H. E. and Gurland, J., 1970. "A Review of Parameters Influencing some Mechanical Properties of Tungsten Carbide-Cobalt Alloys", Powder Metallurgy, V13, pp. 13-31.

Fang, N., Wang, M. and Nedess, C., 1997. "Development of the New-type Indexable Insert with Helical Rake Faces", Proceedings of the Institution of Mechanical Engineers, V211, Part B, pp. 159-164.

Fei, J. and Jawahir, I. S., 1994. "A New Approach for Chip-Form Characterization in Metal Machining”, SME Technical Paper MR94-134.

Finnie, I., 1963. "A Comparison of Stress Strain Behavior in Cutting with that in Other Materials Tests", ASME: International Research in Production Engineering, New York, pp. 76-82.

Finnie, I., 1956. "Review of the Metal Cutting Analyses of the Past Hundred Years", Mechanical Engineering, V78, pp. 715-721.

Fu, H. J., DeVor, R. E. and Kapoor, S. G., 1984. "A Mechanistic Model for the Prediction of Force System in Face Milling Operations", Journal of Engineering for Industry, V106, n1, pp. 81-88.

Ghosh, R., Dillon, O. W. and Jawahir, I. S., 1998. "An Investigation of 3D Curled Chip in Machining. Part 1: A Mechanics Based Analytical Model”, Machining Science and Technology, V2, n1, pp. 91-116.

Grzesik, W., 2008. Advanced Machining Processes of Metallic Materials, Elsevier, Inc.

Grzesik, W. and Kwiatkowska, E., 1997. "An Energy Approach to Chip Breaking when Machining with Grooved Tools", International Journal of Machine Tools and Manufacture, V37, n5, pp. 569-577.

Hagiwara, M., Chen, S. and Jawahir, I. S., 1995. "A Hybrid Predictive Model and Validation for Chip Flow in Contour Finish Turning Operations with Coated Grooved Tools", Journal of Materials Processing Technology, V209, n3, pp. 1417-1427.

Hahn, R. S., 1953. "Some Observations on Chip Curl in the Metal-Cutting Process under Orthogonal Cutting Conditions", Transactions of the ASME, V74, pp. 581-590.

Hardinge Inc, 2010. www.hardingeus.com

Heginbotham, W. B. and Gogia, S. L., 1961. "Metal Cutting and the Built-Up Nose", Proceedings of the Institution of Mechanical Engineers, V175, n18, pp. 892-917. 
Henriksen, E. K., 1955. "Findings and Directions in Chip Breakers Research", SME Technical Paper MR55-104.

Henriksen, E. K., 1954. "Chip Breaking Dimensions are Critical in Taming Chips", American Machinist, V98, n4, pp. 118-124.

Hong, S. Y., Ding, Y. and Ekkens, R. G., 1999. "Improving Low Carbon Steel Breakability by Cryogenic Chip Cooling", International Journal of Machine Tools and Manufacture, V39, pp. 1065-1085.

Hu, R. S., Mathew, P., Oxley, P. L. B. and Young, H. T., 1986. "Allowing for End Cutting Edge Effects in Predicting Forces in Bar Turning with Oblique Machining Conditions", Proceedings of the Institution of Mechanical Engineers, V200, pp. 8999.

ISO 3685:1993 (E), 1993. Tool Life Testing for Single Point Turning Tools, pp. 45-47.

Jawahir, I. S. and Zhang, J. P., 1995. "An Analysis of Chip Curl Development, Chip Deformation and Chip Breaking in Orthogonal Machining", Transactions of NAMRI/SME, V23, pp. 109-114.

Jawahir, I. S. and van Luttervelt, C. A., 1993. "Recent Developments in Chip Control Research and Applications", Annals of the CIRP, V42, n2, pp. 659-685.

Jawahir, I. S., 1993. “Chip Control Literature Database”, Annals of the CIRP, V42, n2, pp. 686-693.

Jawahir, I. S., 1991. “An Investigation of Three-Dimensional Chip Flow in Machining of Steels with Grooved Chip Forming Tool Inserts", Transactions of NAMRI/SME, pp. 222-231.

Jawahir, I. S., 1990. "On the Controllability of Chip Breaking Cycles and Modes of Chip Breaking in Metal Machining”, Annals of the CIRP, V39, n1, pp. 47-51.

Jawahir, I. S. and Oxley, P. L. B., 1988. "New Developments in Chip Control Research Moving Towards Chip Breakability Predictions for Unmanned Manufacture", Proceedings of the International Conference of the ASME, pp. 311-320.

Jawahir, I. S., 1988a. "A Survey and Future Predictions for the Use of Chip Breaking in Unmanned Systems", The International Journal of Advanced Manufacturing Technology, V3, n4, pp. 87- 104.

Jawahir, I. S., 1988b. "The Chip Control Factor in Machinability Assessments: Recent Trends", Journal of Mechanical Working Technology, V17, pp. 213-224. 
Jawahir, I. S., 1988c. "The Tool Restricted Contact Effect as a Major Influencing Factor in Chip Breaking: An Experimental Analysis", Annals of the CIRP, V37, n1, pp. 121126.

Jones, D. G. and Mc Greery, J. F., 1973. "A Study of Preformed Chip Control Devices in Throwaway Carbide Inserts", SME Technical Paper MR73-215.

Katbi, K., 1998. "Top-Form Geometry: The Key to Higher Productivity”, Cutting Tool Engineering, V50, n2.

Kennametal Inc, 2010. Catalog - Turning Tooling 2010.

Kennametal Inc, 1984. "Chip Control Research through High Speed Motion Analysis", The Carbide and Tool Journal, V16, n3, pp. 21-23.

Kharkevich, A. and Venuvinod, P. K., 2002. "Extension of Basic Geometric Analysis of 3-D Chip Forms in Metal Cutting to Chips with Obstacle-induced Deformation”, International Journal of Machine Tools and Manufacture, V42, pp. 201-213.

Kharkevich, A. and Venuvinod, P. K., 1999. "Basic Geometric Analysis of 3-D Chip Forms in Metal Cutting. Part 1: Determining Up-curl and Side-curl Radii”, International Journal of Machine Tools and Manufacture, V39, pp. 751-769.

Kimura, K. and Kawasaki, N., 1981. "Apparatus for Guiding Continuous Chip Formed in Lathing”, US Patent \# 4,271,734.

Klopstock, H., 1925. "Recent Investigations in Turning and Planing and a New Form of Cutting Tool", Transactions of the ASME, V47, pp. 345-377.

Kluft, K., Konig, W., van Luttervelt, C. A., Nakayama, K. and Pekelharing, A. J., 1979. "Present Knowledge of Chip Control", Annals of the CIRP - Keynote Paper, V28, n2, pp. 441-455.

Komanduri, R., 1993. "Machining and Grinding: A Historical Review of Classical Papers", ASME Applied Mechanics Review, V46, n3, pp. 80-132.

Komanduri, R. and Brown, R. H., 1981. "On the Mechanics of Chip Segmentation in Machining", Journal of Engineering for Industry, V103, pp. 33-51.

Korloy Inc, 2010. E-Catalog: Turning, www.korloy.com

Lee, E. H. and Shaffer, B. W., 1951. "Theory of Plasticity Applied to a Problem of Machining", Journal of Applied Mechanics, V18, pp. 405-413. 
Li, F. and Xie, J., 2001. "Metal Cutting Chip Breaking by Lasers", SAE Technical Paper Series, Paper \# 2001-01-0981.

Li, Z. and Rong, Y., 1999. "A Study on Chip Breaking Limits in Machining", Machining Science and Technology, V3, n1, pp. 25-48.

Li, Z., Qu, G., Xu, Y. and Rong, Y., 1997. "Formation and Breaking of Side-Curl Dominated Short-Spiral Chips", Manufacturing Science and Technology, V2, MED Vol 62, pp. 305-312.

Lin, G. C. I. and Oxley, P. L. B., 1972. "Mechanics of Oblique Machining: Predicting Chip Geometry and Cutting Forces from Work Material Properties and Cutting Conditions", Proceedings of the Institution of Mechanical Engineers, V188, pp. 813-820.

Luk, W. K., 1972. "The Direction of Chip Flow in Oblique Cutting”, International Journal of Production Research, V10, n1, pp. 67-76.

Lutov, V. M., 1962. "Selecting the Optimum Size of Chip-breaking Grooves", Machines and Tooling, V33, n7, pp. 27-30.

Machado, A. R. and Wallbank, J., 1994. "The Effect of High Pressure Coolant Jet on Machining", Proceedings of the Institution of Mechanical Engineers. Part B: Journal for Engineering Manufacture, V208, pp. 29-38.

Machinery’s Handbook \#26, 2000. Industrial Press Inc.

Madhavan, V., Chandrasekar, S. and Farris, T. N., 2000. "Machining as a Wedge Indentation", Journal of Applied Mechanics, V67, pp. 128-139.

Marusich, T. D., Brand, C. J. and Thiele, J. D., 2002. “A Methodology for Simulation of Chip Breakage in Turning Processes using an Orthogonal Finite Element Model", Proceedings of the $5^{\text {th }}$ CIRP International Workshop on Modeling of Machining Operation, West Lafayette, USA, pp. 139-148.

Merchant., M. E., 2003. "An Interpretive Review of $20^{\text {th }}$ Century US Machining and Grinding Research", Techsolve Inc, USA.

Merchant, M. E., 1945. "Mechanics of Metal Cutting Process: I. Orthogonal Cutting and a Type 2 Chip", Journal of Applied Physics, V 16, n 5, pp. 267-275.

Merchant, M. E., 1944. "Basic Mechanics of the Metal Cutting Process", Journal of Applied Mechanics, V 11, pp. 168-175. 
Nakatani, S., 1973. "Application Characteristics of Throw Away Insert Tools", SME Technical Paper MR73-910.

Nakayama, K. and Arai, M., 1992. "Comprehensive Chip Form Classification Based on the Cutting Mechanism”, Annals of the CIRP, V41, n1, pp. 71-74.

Nakayama, K., Arai, M., Kondo, T. and Suzuki, H., 1981. "Cutting Tool with Curved Rake Face - A Means for Breaking Thin Chips", Annals of the CIRP, V30, n1, pp. 58.

Nakayama, K. and Arai, M., 1980. "The Breakability of Chip in Metal Machining”, International Conference on Manufacturing Engineering, August, Melbourne, AUS, pp. 6-10.

Nakayama, K. and Ogawa, M., 1978. "Basic Rules on the Form of Chip in Metal Cutting", Annals of the CIRP, V27, n1, pp. 17-21.

Nakayama, K. and Arai, M., 1977. "Roles of Brittleness of Work Material in Metal Cutting", Proceedings of the International Symposium on Influence of Metallurgy on Machinability of Steel, Sept 26-28, Tokyo, Japan, pp. 421-432.

Nakayama, K., 1972. "Origins of the Side-curl in Chip in Metal Machining", Bulletin of the Japanese Society of Precision Engineering, V6, n3, pp. 99-100.

Nakayama, K., 1963. "Pure Bending Test of Chip - An Approach to the Prediction of Cutting Force", Bulletin of the Faculty of Engineering - Yokohama National University, V12, pp. 1-14.

Nakayama, K., 1962. "A Study on Chip-breaker", Bulletin of the Japanese Society of Mechanical Engineering, V5, n17, pp. 142-150.

Nedess, C. and Hintze, W., 1989. "Characteristic Parameters of Chip Control in Turning Operations with Indexable Inserts and Three Dimensionally Shaped Chip Formers", Annals of the CIRP, V38, n1, pp. 75-59.

Okoshi, M. and Kawata, K., 1967. "Effects of Curvature of Work Surface on Metal Cutting", Annals of the CIRP, V15, pp. 393-403.

Okushima, K., Hoshi, T. and Fujinawa, T., 1960. "On the Behaviour of Chip in Steel Cutting (Part 2. In the Case of Parallel Type Chip Breaker)", Bulletin of the Japanese Society of Mechanical Engineers, V3, n10, pp. 199-205.

Okushima, K. and Minato, K., 1959. "On the Behavior of Chip in Steel Turning”, Bulletin of JSME, V2, n5, pp. 58-64. 
Ostwald, P. F., 1967. "Dynamic Chip Breaking - Can it Overcome the Surface Finish Problem", SME Technical Paper MR67-228.

Pal, A. K. and Koenigsberger, F., 1968. "Some Aspects of the Oblique Cutting Process", International Journal of Machine Tool Design and Research, V8, pp. 4557.

Palmer, W. B. and Yeo, R. C. K., 1963. "Metal Flow Near the Tool point During Orthogonal Cutting with a Blunt Tool", Advances in Machine Tool Design and Research: Proceedings of the 4th International M.T.D.R. Conference, the Manchester College of Science \& Technology, September 1963, pp. 61-71.

Palmer, W. B. and Oxley, P. L. B., 1959. "Mechanics of Orthogonal Machining", Proceedings of the Institution of Mechanical Engineers, V173, n24, pp. 623-654.

Pekelharing, A. J., 1964. "Why and How Does the Chip Curl and Break", Annals of the CIRP, V12, n1, pp. 144-147.

Piispanen, V., 1948. "Theory of Formation of Metal Chips", Journal of Applied Physics, V19, pp. 876-881.

Ponkshe, G. R., 1967. "A New Explanation of the Phenomenon of Chip Curling During Machining", Transactions of the ASME: Journal of Engineering for Industry, V89, pp. 376-379.

Rolt., L. T. C., 1965. A Short History of Machine Tools, The MIT Press, Massachussets.

Roth, R. N. and Oxley, P. L. B., 1972. "A Slip Line Field Analysis for Orthogonal Machining Based on Experimental Flow Fields", Journal of Mechanical Engineering Science, V14, pp. 85-97.

Russell, J. K. and Brown, R. H., 1966. "The Measurement of Chip Flow Direction”, International Journal of Machine Tool Design and Research, V6, pp. 129-138.

Sandvik Coromant, 2009a. Main Catalog, AB Sandvik Coromant.

Sandvik Coromant, 2009b. Technical Guide, AB Sandvik Coromant.

Sandvik Coromant, 2005. Technical Guide, AB Sandvik Coromant.

Sandvik Coromant, 1996. Modern Metal Cutting, AB Sandvik Coromant.

Schneider, G., 2002. Cutting Tool Applications, GMRS Associates. 
SECO Tools, 2010. Web Catalog: Turning. http://ecat.secotools.com/

Seethaler, R. J. and Yellowley, I., 1996. "An Upper Bound Cutting Model for Oblique Cutting Tools with a Nose Radius", International Journal of Machine Tools and Manufacture, V37, n2, pp. 119-134.

Sekhon, G. S. and Chenot, J. L., 1993. "Numerical Simulation of Continuous Chip Formation during Non-Steady Orthogonal Cutting", Engineering Computations, V10, n1, pp.31- 48 .

Shaw, M. C., 2005. Metal Cutting Principles, $2^{\text {nd }}$ Edition, Oxford University Press.

Shaw, M. C., Cook, N. H. and Smith, P. A., 1952. "The Mechanics of ThreeDimensional Cutting Operations", Transactions of the ASME, V74, n6, pp. 10551064.

Shinozaki, N. and Hirota, H., 1970. "Chip Breaking by Rolling Tool”, SME Technical Paper MR70-250.

Sikdar, C., Babu, S. S., Chattopadhyay, A. B. and Venkatesh, V. C., 1991. "Effect of Bevelling of Carbide Turning Inserts on Chip Formation and Cutting Forces", Journal of Materials Processing Technology, V28, pp. 15-24.

Skelton, R. C. and Tobias, S. A., 1962. "Survey of Research on Cutting with Oscillating Tools", Proceedings of the $3^{\text {rd }}$ International Conference on Machine Tool Design and Research, Birmingham, UK, pp. 5-16.

SME Video, 2001. Fundamental Manufacturing Processes (Electronic Resources), DVD Disc 8.

SME, 1996. Tool and Manufacturing Engineer's Handbook, Vol-1.

Smith, S., Woody, B., Barkman, W. and Tursky, D., 2009. "Temperature Control and Machine Dynamics in Chip Breaking using CNC Toolpaths", Annals of the CIRP, V58, pp. 97-100.

Spaans, C. and Hovinga, H. J., 1971. "Chip Breaking in Finish Turning of Varying Feed with High Surface Quality Maintained During Entire Tool Life", SME Technical Paper MR71-904.

Spaans, C. and van Geel, P. F. H. C., 1970. "Break Mechanisms in Cutting with a Chipbreaker", Annals of the CIRP, V18, pp. 87-92. 
Spaans, C., 1970. "A Systematic Approach to Three Dimensional Chip Curl, Chip Breaking and Chip Control", SME Technical Paper, MR70-241.

Stabler, G. V., 1964. "The Chip Flow Law and its Consequences", Proceedings of Fifth International Machine Tool Design and Research Conference, Birmingham, UK, pp. 243-251.

Stabler, G. V., 1951. "The Fundamental Geometry of Cutting Tools", Proceedings of the Institution of Mechanical Engineers, V165, pp. 14-26.

Stephenson, D. A. and Agapiou, J. S., 2006. Metal Cutting Theory and Practice, CRC Taylor and Francis.

Strenkowski, J. S. and Moon, K. J., 1990. "Finite Element Prediction of Chip Geometry and Tool/Workpiece Temperature Distributions in Orthogonal Metal Cutting", ASME Journal of Engineering for Industry, V112, pp. 313-318.

Strenkowski, J. S. and Carroll, J. T., 1985. "A Finite Element Model of Orthogonal Metal Cutting", ASME Journal of Engineering for Industry, V107, pp. 346-354.

Subbiah, S. and Melkote, S. N., 2007. "Evidence of Ductile Tearing Ahead of the Cutting Tool and Modeling the Energy Consumed in Material Separation in MicroCutting”, Journal of Engineering Materials and Technology, V129, n2, pp. 321-331.

Subramani, G., Suvada, R., Kapoor, S. G., DeVor, R. E. and Meingast, W., 1987. “A Model for Prediction of Force System for Cylinder Boring Process", Proceedings of the $15^{\text {th }}$ NAMRC, Bethlehem, PA, pp. 439-446.

Takeyama, H., and Usui, E., 1958. "The Effect of Tool-Chip Contact Area in Metal Machining", Transactions of the ASME, V80, pp. 1089-1096.

Tungalloy Corp, 2010. Web Catalog: www.tungaloy.com

Usui, E. and Hirota, A., 1978. "Analytical Prediction of Three Dimensional Cutting Process. Part 2: Chip Formation and Cutting Force with Conventional Single-Point Tool”, Transactions of ASME: Journal of Engineering for Industry, V100, n5, pp. 229-235.

Usui, E. and Takeyama, H., 1960. "A Photoelastic Analysis of Machining Stresses", ASME Transactions: Journal of Engineering for Industry, V82, n4, pp. 303-308.

van Luttervelt, C. A., 1976. "Chip Formation in Machining Operation at Small Diameter", Annals of the CIRP, V25, n1, pp. 71-76. 
Venkatesh, V. C., Yee, C., Quinto, D. T. and Hoy, D. E. P., 1991. "Performance studies of Uncoated, CVD Coated and PVD Coated Carbides in Turning and Milling", Annals of the CIRP, V40, n1, pp. 545-550.

Venuvinod, P. K. and Lau, W. S., 1977. "On the Estimation of Chip Flow Angle in Oblique Cutting”, Microtecnic 2, pp. 32-35.

von Turkovich, B. F., 1981. "Metallurgy - A Foundation for Understanding the Art of Machining", Proceedings of the Winter Annual Meeting of the ASME, Washington D C, USA, PED-Vol 3, pp. 1-9.

Wallace, P. W. and Boothroyd, G., 1964. "Tool Forces and Tool Chip Friction in Orthogonal Machining”, Journal of Mechanical Engineering Science, V6, n1, pp. 7487.

Wang, J. and Mathew, P., 1995. "Development of a General Tool Model for Turning Operations Based on a Variable Flow Stress Theory", International Journal of Machine Tool Manufacture, V35, n1, pp. 71-90.

Wirfelt, S., 1969. "What Form for Chipbreakers", Metalworking Production, Magazine Issue March 5, 1969, pp. 39-40.

Worthington, B., 1975. "The Effect of Rake Face Configuration on the Curvature of the Chip in Metal Machining", International Journal of Machine Tool Design and Research, V15, pp. 223-229.

Worthington, B. and Redford, A. H., 1973. "Chip Curl and the Action of the Groove Type Chip Former", International Journal of Machine Tool Design and Research, V13, pp. 257-270.

Young, H. T., Mathew, P. and Oxley, P. L. B., 1987. "Allowing for Nose Radius Effects in Predicting the Chip Flow Direction and Cutting Forces in Bar Turning", Proceedings of the Institution of Mechanical Engineers, V201, pp. 213- 226.

Zhang, X. D., Lee, L. C. and Seah, K. W. H., 1995. "Knowledge Base for Chip Management System", Journal of Materials Processing Technology, V48, pp. 215-221.

Zorev, N.N., 1966. Metal Cutting Mechanics, Pergamon Press. 


\section{APPENDIX A}

\section{CUTTING KINEMATICS AND TOOL GEOMETRY}

In general, most representations of the cutting process include a simplified two dimensional (2D) representation as shown in Figure 2.1. With the exclusion of a few processes, e.g., planing, shaping and broaching, the vast majority of the cutting processes are carried out using complex three dimensional (3D) tool geometries. Figure A.1 shows the elements of tool edge geometry.

The idealized 2D representation of the cutting process corresponds to events confined to only a small segment of the cutting edge but nevertheless, accurately captures the physics of the entire process at a fundamental level. The 3D representation of both the orthogonal and oblique cutting is shown in Figure A.2. To account for the many different tool orientations that are possible, in practice, standardized coordinate systems are used to define all the angles of the cutting tool. A good account of the geometric foundations of tool design can be found in Boothroyd and Knight (2006) and the SME: Tool and Manufacturing Engineer's Handbook (1996). In keeping with the context of this research, the inclined geometry of the cutting edge is illustrated for visualization in Figure A.3. A more comprehensive reference can be found elsewhere (Endres, 2005). 


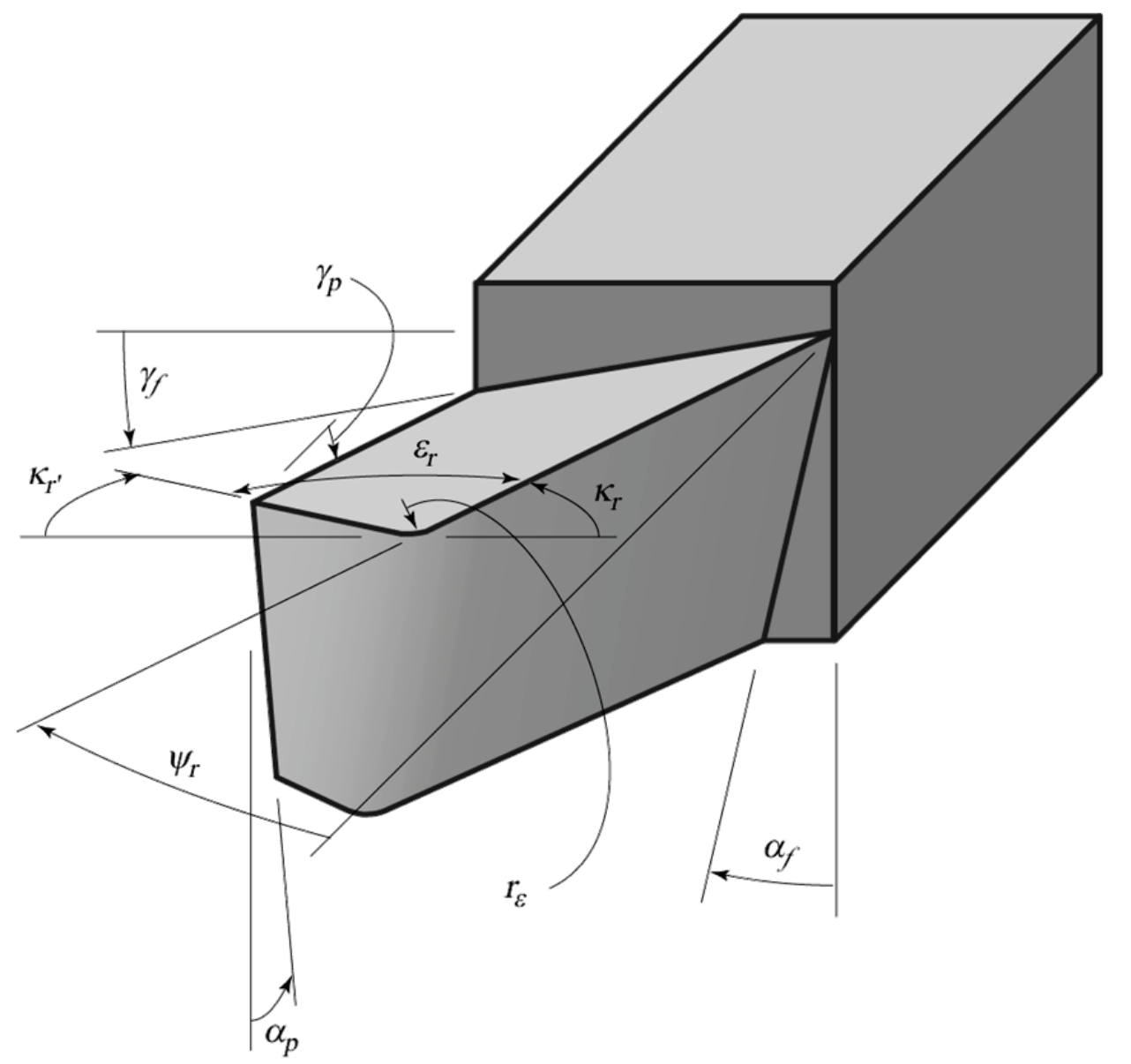

$\gamma_{p}$ - Side rake angle

$\gamma_{f}$ - Back rake angle

$r_{\varepsilon}$ - Tool corner radius

$\kappa_{r}$ - Major cutting edge angle

$\kappa_{r}$, - Minor cutting edge angle

$$
\begin{gathered}
\psi_{r}-\text { Tool lead angle } \\
\alpha_{p}-\text { Minor edge flank clearance angle } \\
\alpha_{f} \text { - Major edge flank clearance angle } \\
\varepsilon_{r}-\text { Tool corner angle }
\end{gathered}
$$

Figure A.1: Tool geometry nomenclature. (Endres, 2005)

Cutting tools used in practice are also designed with a corner radius blending the major and minor cutting edges. The primary function of the corner radius is to provide edge strength. Generally, the corner radius as a cutting variable can either be ignored for pur- 
poses of idealization or included in the study of chip control. Some researchers (Zorev, 1966; Astakhov, 1999) have classified the cutting process as free (without a corner radius involved in cutting) or non-free (with the corner radius involved in cutting) cutting. Note that the free or non-free configuration as shown in Figure A.4 can be used in the context of both orthogonal and oblique cutting. The main difference between free and non-free cutting, especially when applied to chip flow problems, is the resulting projected chip area shown in Figure A.4. The inclusion of the corner radius results in a thinner chip at the tool corner radius region, due to which, the orientation of the resultant cutting force is changed. Consequently, the chip flow angle is also altered. The kinematics of the free and non-free cutting process and its effects on chip flow angle has been very well explained by Astakhov (1999).

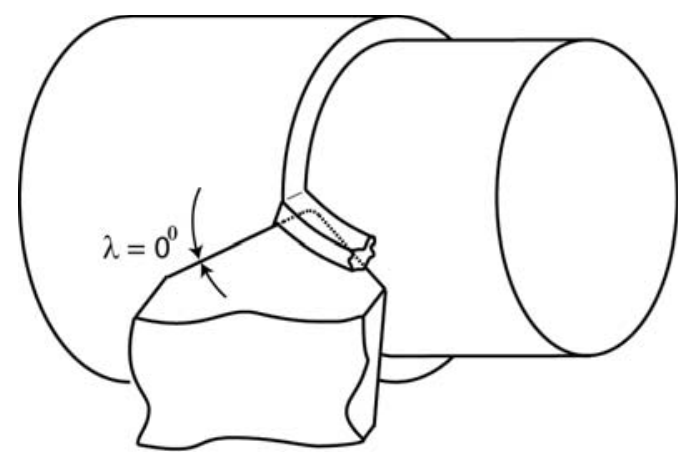

(a)

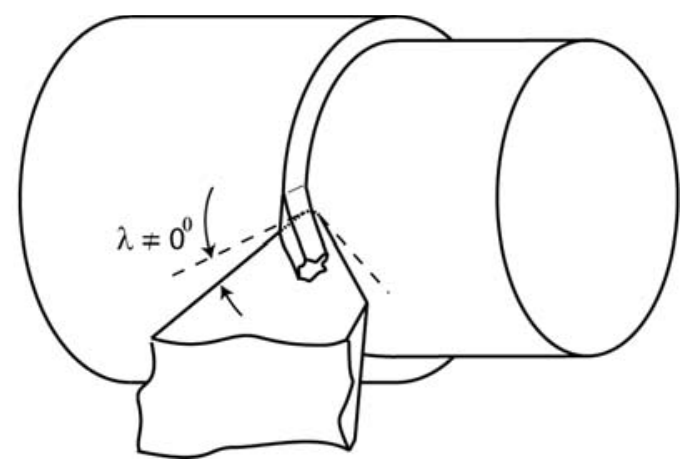

(b)

Figure A.2: 3D representation of (a) Orthogonal cutting (b) Oblique cutting. 

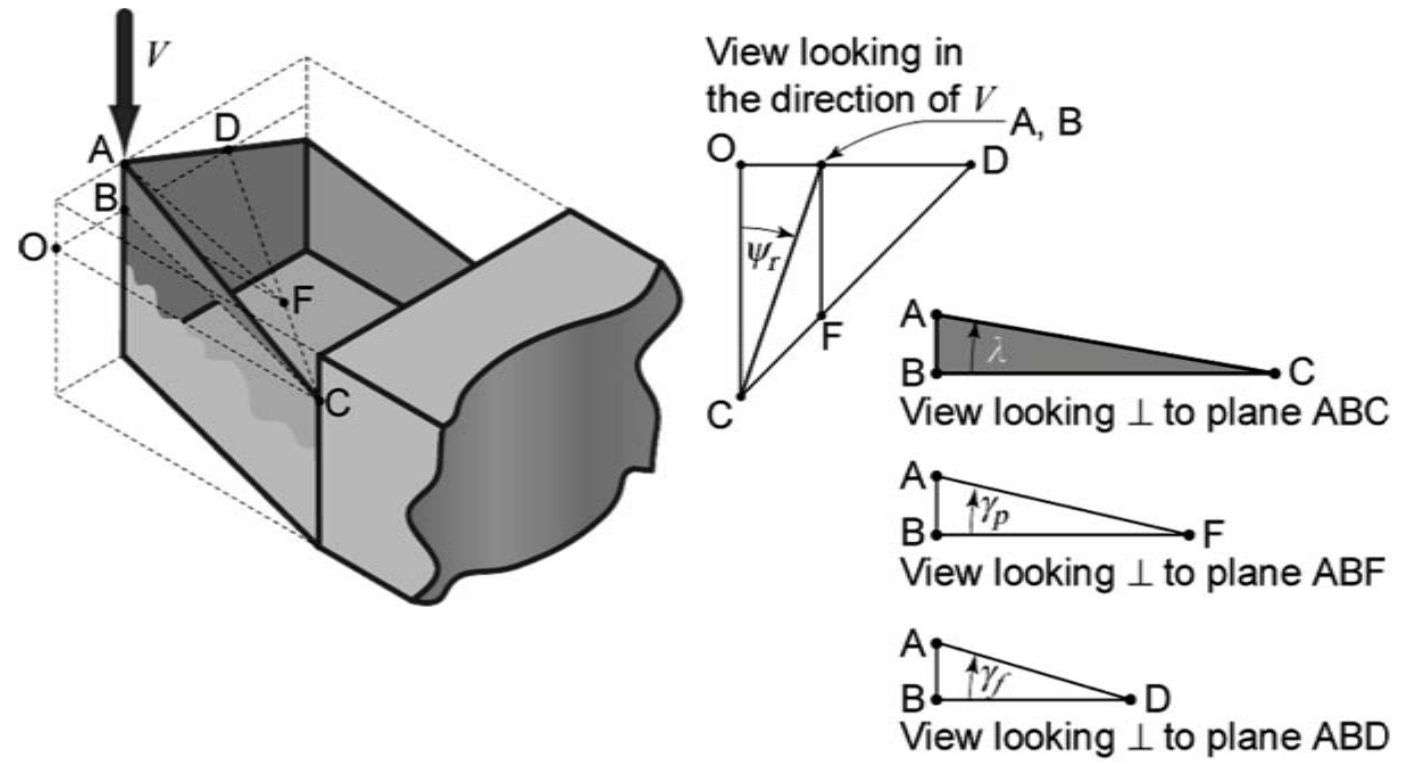

Figure A.3: Visualization of the inclination angle. (Endres, 2005)
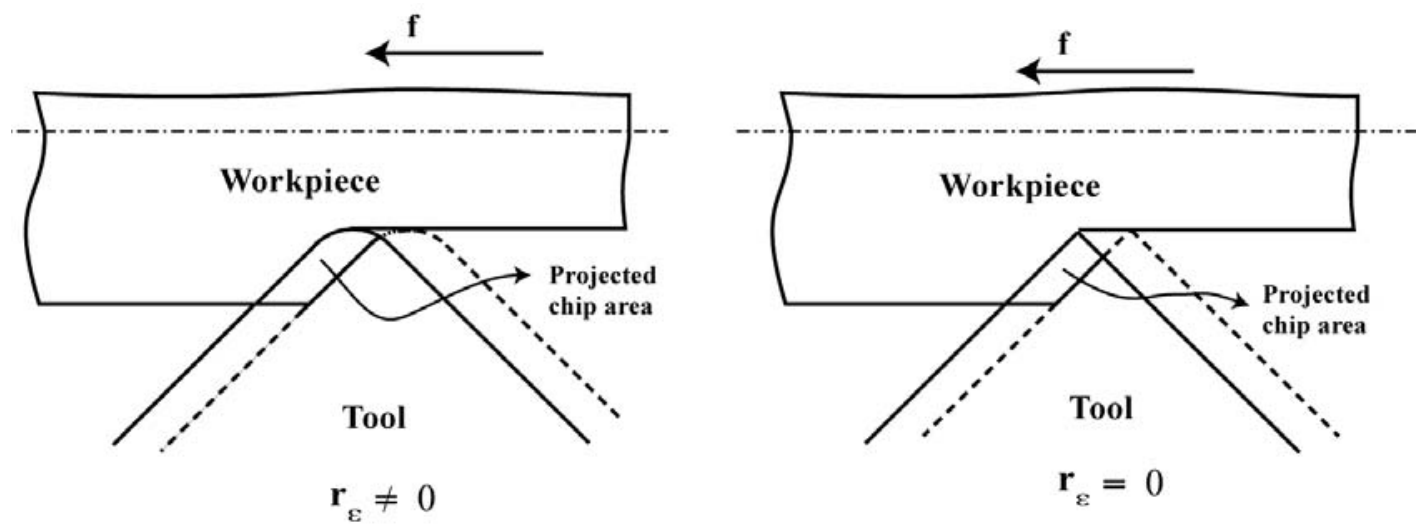

Figure A.4: Illustrative definition of (left) Free cutting (right) Non-free cutting. 


\section{APPENDIX B}

\section{CHARACTERIZATION OF THE CUTTING TOOL}

\section{EDGE}

In Chapter 3, a methodology was presented on the development of a working prototype using the model. To validate the tool geometry with the model predicted values, the developed prototype tool was characterized by measuring the various tool geometries. The measurements were made based on photographs of the different tool geometries.

1. The tool top face was measured by photographing the rake face using the Nikon D70 camera. Both the horizontal and vertical dimensions of the photographic image were calibrated using a machinists scale which was also pictured at the same focal distance as the tool rake face. The method used for calibration of the photographic images is shown in Figure B.1. Based on the images it is observed that the actual corner radius is smaller than that of the chosen insert size (S433 or S432). This is due to the material removed on the rake face combined with the flank angle of the tool. Actual measurements obtained by the calibration process for both the S433 and S432 inserts are shown in Figures B.2 - B.3.

2. Measurement of the varying edge inclination was also done in a similar fashion. Since the model generated coordinates were mapped in the insert coordinate system, the inclination angle was also measured as a series of $\left(Y_{i}, Z_{i}\right)$ coordinates (see Chapter 
3 for details) in the same insert coordinate system. The measurements were based on images of the tool edge obtained from a standard tool makers microscope (TMM) equipped with a camera and axis readouts. The $Y_{i}$ coordinates used were taken directly from the model predicted values and the corresponding $Z_{i}$ was measured. One such measurement is shown in Figure B.4. The lengths of the red lines correspond to the image calibrated value of the model generated $\left(Y_{i}, Z_{i}\right)$ coordinates. It can be seen that the $Z_{i}$ values as measured are very close to the model values indicating that the edge inclination obtained from the tool making process matched well with that predicted theoretically. In general, the error on the $Z_{i}$ values ranged between $0.003-0.010$ ". It is presumed that the error could be attributed to either the machining process (tool deflection) or from the measurement process itself.

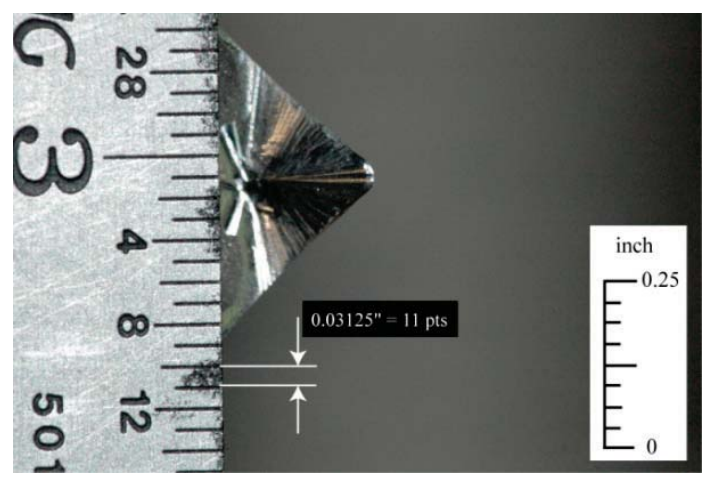

(a)

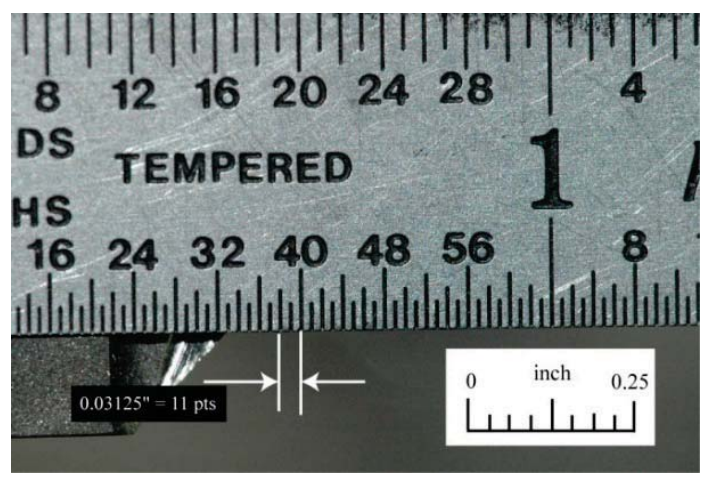

(b)

Figure B.1: Method for photographic calibration (a) Horizontal direction (b) Vertical direction. 


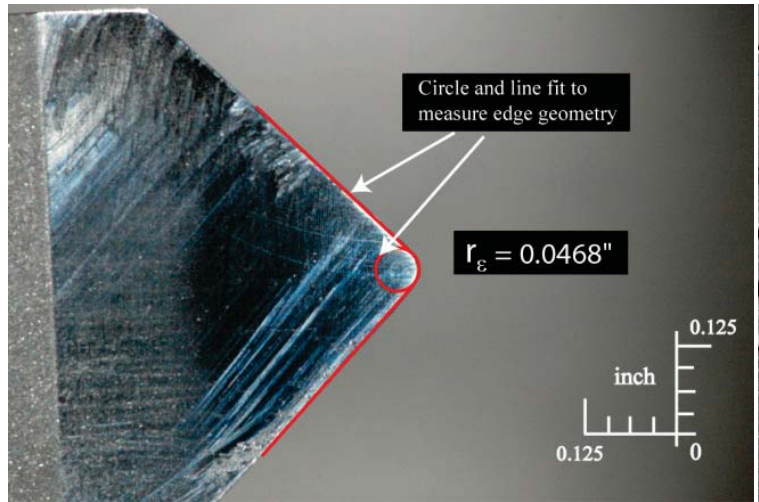

(a)

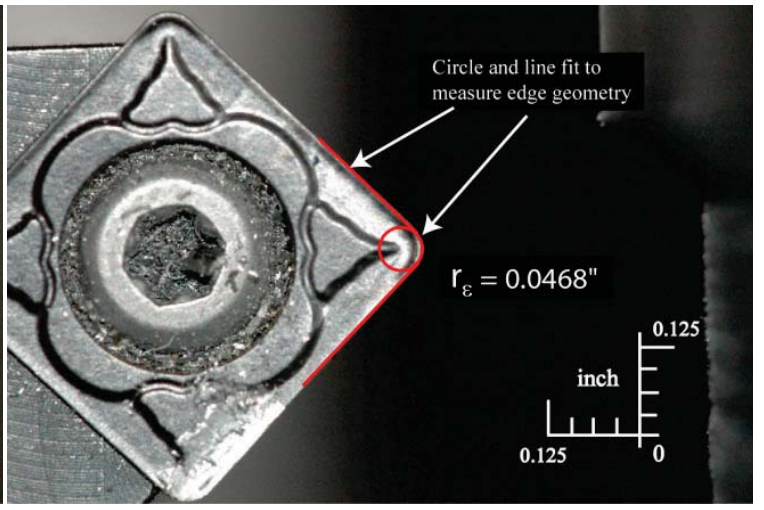

(b)

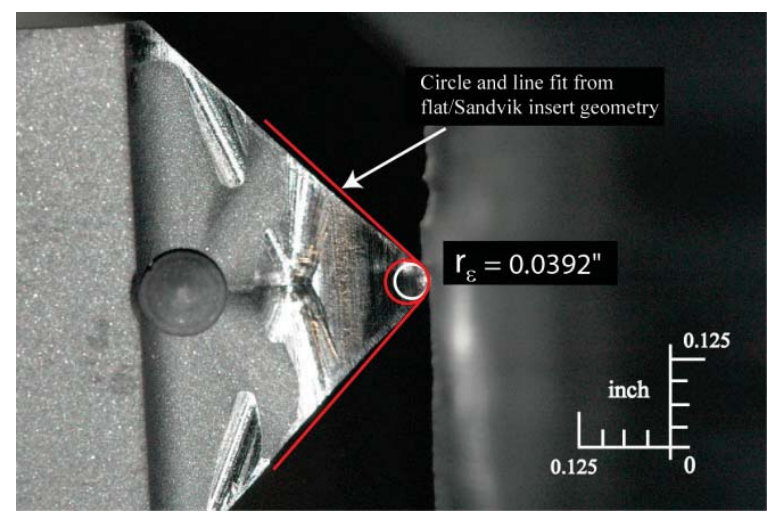

(c)

Figure B.2: Tool corner radius measurement for (a) Flat tool with S-433 configuration (b) Sandvik S-433 insert (c) UCB tool with S-433 configuration. 


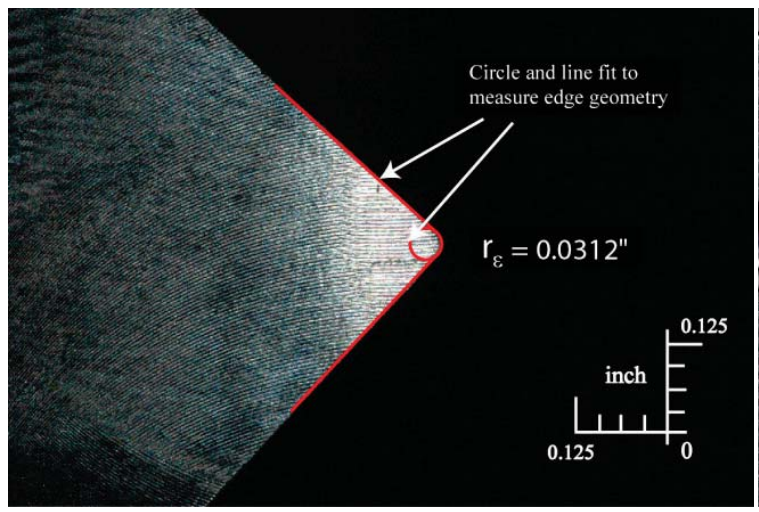

(a)

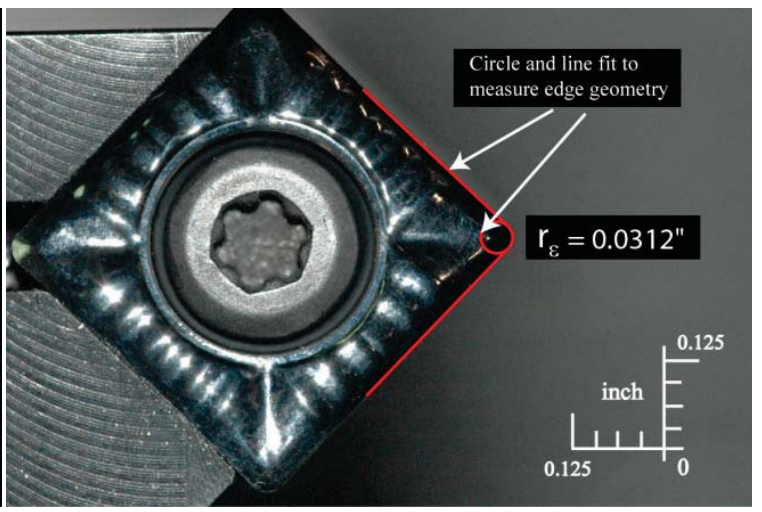

(b)

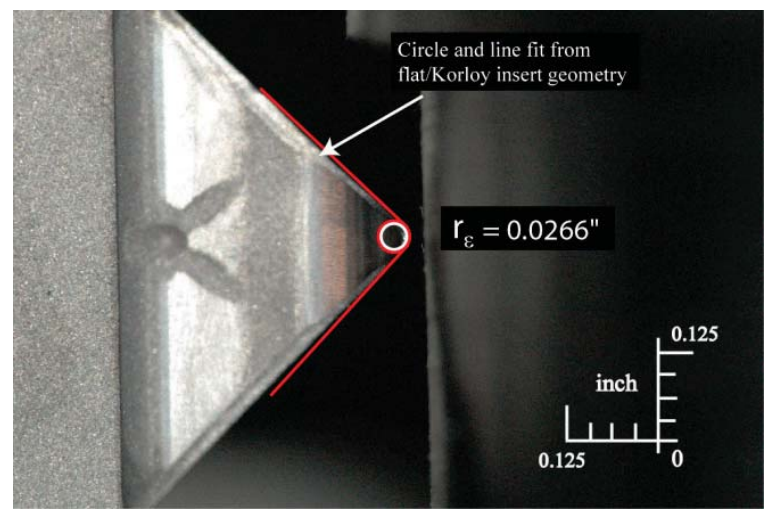

(c)

Figure B.3: Tool corner radius measurement for (a) Flat tool with S-432 configuration (b) Korloy S-432 insert (c) UCB tool with S-432 configuration. 

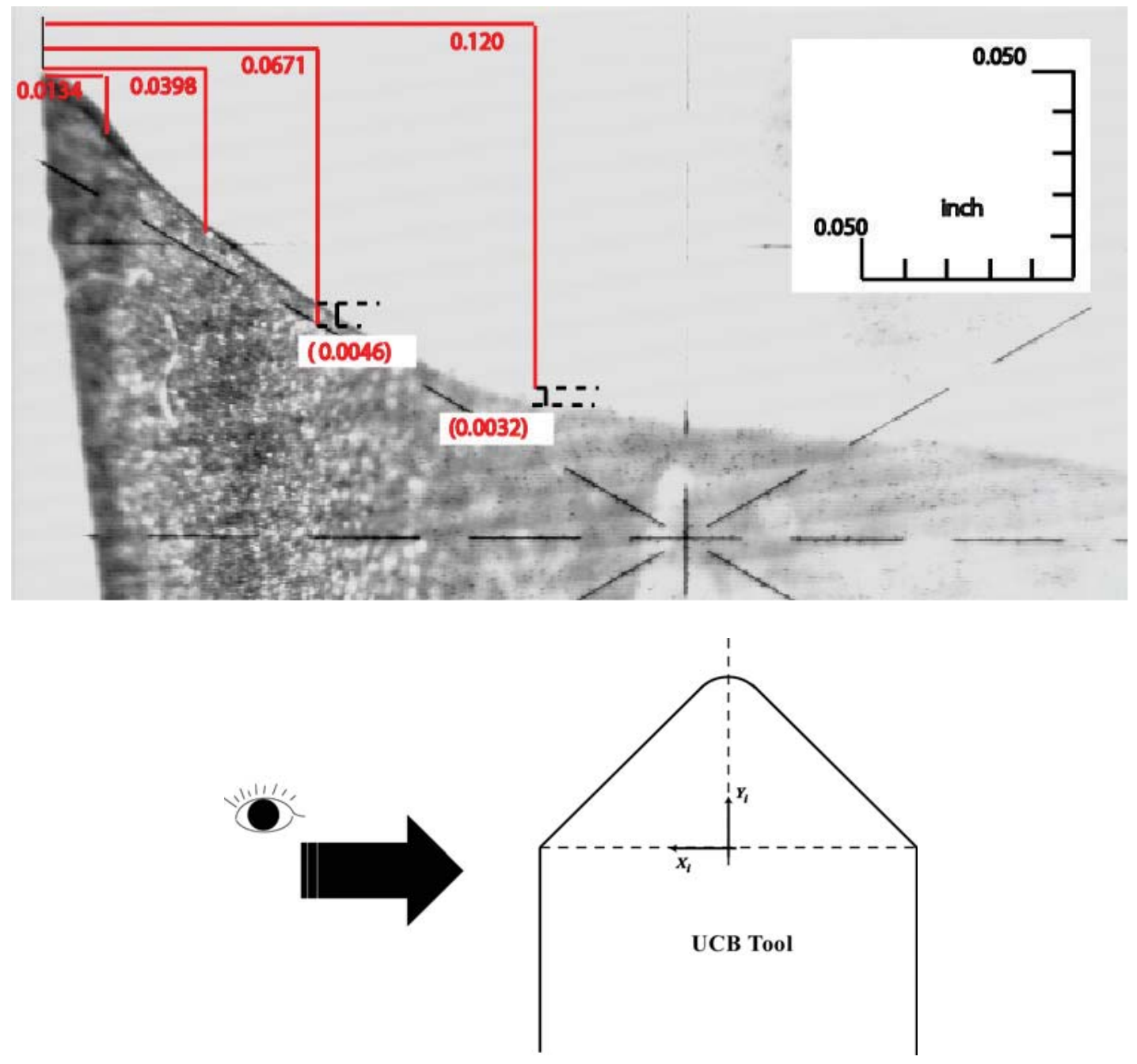

Figure B.4: (top) Characterization of the edge inclination with (bottom) Schematic of the direction of viewing. 


\section{APPENDIX C}

\section{DETAILS OF EXPERIMENTAL RUN ORDER}

(this page intentionally left blank) 
Table C.1: Run order for cutting experiments. Work material: AISI 1018, $\psi_{r}=4^{\circ}$.

\begin{tabular}{|c|c|c|c|c|c|}
\hline $\begin{array}{l}\text { Material: } \\
\text { Lead Angle, } \psi \text { (deg): } \\
\text { Cutting Speed (sfm): }\end{array}$ & $\begin{array}{l}\text { AISI } 1018 \\
45 \\
110\end{array}$ & & & & \\
\hline \multirow{2}{*}{ Tool Type } & \multirow{2}{*}{$\begin{array}{l}\text { Depth of Cut } \\
\text { (inch) }\end{array}$} & \multirow{2}{*}{$\begin{array}{l}\text { Feed } \\
\text { (ipr) }\end{array}$} & \multicolumn{3}{|c|}{ Test Sequence } \\
\hline & & & Replication \# 1 & Replication \# 2 & Replication \# 3 \\
\hline \multirow{9}{*}{ Flat Rake Face } & \multirow{3}{*}{0.025} & 0.005 & 25 & 7 & 17 \\
\hline & & 0.008 & 20 & 2 & 18 \\
\hline & & 0.014 & 23 & 5 & 16 \\
\hline & \multirow{3}{*}{0.08} & 0.005 & 17 & 10 & 5 \\
\hline & & 0.008 & 13 & 11 & 8 \\
\hline & & 0.014 & 10 & 12 & 3 \\
\hline & \multirow{3}{*}{0.12} & 0.005 & 6 & 23 & 26 \\
\hline & & 0.008 & 1 & 27 & 25 \\
\hline & & 0.014 & 9 & 20 & 27 \\
\hline \multirow{9}{*}{ Sandvik } & \multirow{3}{*}{0.025} & 0.005 & 19 & 3 & 10 \\
\hline & & 0.008 & 21 & 1 & 14 \\
\hline & & 0.014 & 24 & 6 & 12 \\
\hline & \multirow{3}{*}{0.08} & 0.005 & 15 & 16 & 7 \\
\hline & & 0.008 & 11 & 17 & 1 \\
\hline & & 0.014 & 16 & 18 & 6 \\
\hline & \multirow{3}{*}{0.12} & 0.005 & 4 & 24 & 22 \\
\hline & & 0.008 & 8 & 19 & 24 \\
\hline & & 0.014 & 2 & 26 & 19 \\
\hline \multirow{9}{*}{$\mathrm{UCB}$} & \multirow{3}{*}{0.025} & 0.005 & 27 & 9 & 11 \\
\hline & & 0.008 & 26 & 8 & 13 \\
\hline & & 0.014 & 22 & 4 & 15 \\
\hline & \multirow{3}{*}{0.08} & 0.005 & 14 & 13 & 2 \\
\hline & & 0.008 & 12 & 14 & 9 \\
\hline & & 0.014 & 18 & 15 & 4 \\
\hline & \multirow{3}{*}{0.12} & 0.005 & 3 & 25 & 20 \\
\hline & & 0.008 & 7 & 21 & 23 \\
\hline & & 0.014 & 5 & 22 & 21 \\
\hline
\end{tabular}

NOTE: Strike through numbers in Replication \#3 indicate tool breakage conditions. 
Table C.2: Run order for cutting experiments. Work material: AISI 1018, $\psi_{r}=1^{\circ}$.

\begin{tabular}{|c|c|c|c|c|c|}
\hline $\begin{array}{l}\text { Material: } \\
\text { Lead Angle, } \psi \text { (deg): } \\
\text { Cutting Speed (sfm): }\end{array}$ & $\begin{array}{l}\text { AISI } 1018 \\
15 \\
110\end{array}$ & & & & \\
\hline \multirow{2}{*}{ Tool Type } & \multirow{2}{*}{$\begin{array}{l}\text { Depth of Cut } \\
\text { (inch) }\end{array}$} & \multirow{2}{*}{$\begin{array}{l}\text { Feed } \\
\text { (ipr) }\end{array}$} & \multicolumn{3}{|c|}{ Test Sequence } \\
\hline & & & Replication \# 1 & Replication \# 2 & Replication \# 3 \\
\hline \multirow{9}{*}{ Flat Rake Face } & \multirow{3}{*}{0.025} & 0.005 & 14 & 6 & - \\
\hline & & 0.008 & 18 & 7 & - \\
\hline & & 0.014 & 10 & 4 & - \\
\hline & \multirow{3}{*}{0.08} & 0.005 & 2 & 13 & - \\
\hline & & 0.008 & 7 & 16 & - \\
\hline & & 0.014 & 5 & 15 & - \\
\hline & \multirow{3}{*}{0.12} & 0.005 & 24 & - & - \\
\hline & & 0.008 & 27 & - & - \\
\hline & & 0.014 & 25 & - & - \\
\hline \multirow{9}{*}{ Sandvik } & \multirow{3}{*}{0.025} & 0.005 & 11 & 9 & 18 \\
\hline & & 0.008 & 15 & 2 & 13 \\
\hline & & 0.014 & 16 & 3 & 16 \\
\hline & \multirow{3}{*}{0.08} & 0.005 & 3 & 10 & 11 \\
\hline & & 0.008 & 8 & 18 & 8 \\
\hline & & 0.014 & 4 & 14 & 10 \\
\hline & \multirow{3}{*}{0.12} & 0.005 & 19 & 23 & 2 \\
\hline & & 0.008 & 22 & 19 & 5 \\
\hline & & 0.014 & 20 & 22 & 1 \\
\hline \multirow{9}{*}{$\mathrm{UCB}$} & \multirow{3}{*}{0.025} & 0.005 & 13 & 1 & 14 \\
\hline & & 0.008 & 17 & 8 & 17 \\
\hline & & 0.014 & 12 & 5 & 15 \\
\hline & \multirow{3}{*}{0.08} & 0.005 & 1 & 12 & 7 \\
\hline & & 0.008 & 9 & 17 & 12 \\
\hline & & 0.014 & 6 & 11 & 9 \\
\hline & \multirow{3}{*}{0.12} & 0.005 & 23 & 20 & 4 \\
\hline & & 0.008 & 21 & 21 & 3 \\
\hline & & 0.014 & 26 & 24 & 6 \\
\hline
\end{tabular}

NOTE: Partial/No data for Flat insert due to tool breakage. 
Table C.3: Run order for cutting experiments. Work material: AL 2024, $\psi_{r}=4^{\circ}$.

\begin{tabular}{|c|c|c|c|c|c|}
\hline \multirow{3}{*}{$\begin{array}{l}\text { Material: } \\
\text { Lead Angle, } \psi(\mathrm{deg}) \text { : } \\
\text { Cutting Speed (sfm): }\end{array}$} & \multicolumn{5}{|l|}{ AL 2024} \\
\hline & \multicolumn{5}{|l|}{45} \\
\hline & \multicolumn{5}{|l|}{225} \\
\hline \multirow{2}{*}{ Tool Type } & \multirow{2}{*}{$\begin{array}{l}\text { Depth of Cut } \\
\text { (inch) }\end{array}$} & \multirow{2}{*}{$\begin{array}{l}\text { Feed } \\
\text { (ipr) }\end{array}$} & \multicolumn{3}{|c|}{ Test Sequence } \\
\hline & & & Replication \# 1 & Replication \# 2 & Replication \# 3 \\
\hline \multirow{9}{*}{ Flat Rake Face } & \multirow{3}{*}{0.025} & 0.004 & - & - & - \\
\hline & & 0.008 & - & - & - \\
\hline & & 0.012 & - & - & - \\
\hline & \multirow{3}{*}{0.05} & 0.004 & - & - & - \\
\hline & & 0.008 & - & - & - \\
\hline & & 0.012 & - & - & - \\
\hline & \multirow{3}{*}{0.1} & 0.004 & - & - & - \\
\hline & & 0.008 & - & - & - \\
\hline & & 0.012 & - & - & - \\
\hline \multirow{9}{*}{ Korloy } & \multirow{3}{*}{0.025} & 0.004 & 2 & 12 & 13 \\
\hline & & 0.008 & 5 & 9 & 17 \\
\hline & & 0.012 & 6 & 11 & 15 \\
\hline & \multirow{3}{*}{0.05} & 0.004 & 8 & 4 & 9 \\
\hline & & 0.008 & 11 & 1 & 7 \\
\hline & & 0.012 & 9 & 6 & 11 \\
\hline & \multirow{3}{*}{0.1} & 0.004 & 14 & 17 & 3 \\
\hline & & 0.008 & 17 & 15 & 6 \\
\hline & & 0.012 & 15 & 14 & 2 \\
\hline \multirow{9}{*}{ UCB } & \multirow{3}{*}{0.025} & 0.004 & 1 & 7 & 16 \\
\hline & & 0.008 & 4 & 10 & 14 \\
\hline & & 0.012 & 3 & 8 & 18 \\
\hline & \multirow{3}{*}{0.05} & 0.004 & 12 & 5 & 10 \\
\hline & & 0.008 & 7 & 2 & 8 \\
\hline & & 0.012 & 10 & 3 & 12 \\
\hline & \multirow{3}{*}{0.1} & 0.004 & 18 & 13 & 1 \\
\hline & & 0.008 & 16 & 16 & 5 \\
\hline & & 0.012 & 13 & 18 & 4 \\
\hline
\end{tabular}

NOTE: No data for Flat insert due to tool breakage. 
Table C.4: Run order for cutting experiments. Work material: AL 2024, $\psi_{r}=15^{0}$.

\begin{tabular}{|c|c|c|c|c|c|}
\hline $\begin{array}{l}\text { Material: } \\
\text { Lead Angle, } \psi \text { (deg): } \\
\text { Cutting Speed (sfm): }\end{array}$ & $\begin{array}{l}\text { AL } 2024 \\
15 \\
225\end{array}$ & & & & \\
\hline \multirow{2}{*}{ Tool Type } & \multirow{2}{*}{$\begin{array}{l}\text { Depth of Cut } \\
\text { (inch) }\end{array}$} & \multirow{2}{*}{$\begin{array}{l}\text { Feed } \\
\text { (ipr) }\end{array}$} & \multicolumn{3}{|c|}{ Test Sequence } \\
\hline & & & Replication \# 1 & Replication \# 2 & Replication \# 3 \\
\hline \multirow{9}{*}{ Flat Rake Face } & \multirow{3}{*}{0.025} & 0.004 & - & - & - \\
\hline & & 0.008 & - & - & - \\
\hline & & 0.012 & - & - & - \\
\hline & \multirow{3}{*}{0.05} & 0.004 & - & - & - \\
\hline & & 0.008 & - & - & - \\
\hline & & 0.012 & - & - & - \\
\hline & \multirow{3}{*}{0.1} & 0.004 & - & - & - \\
\hline & & 0.008 & - & - & - \\
\hline & & 0.012 & - & - & - \\
\hline \multirow{9}{*}{ Korloy } & \multirow{3}{*}{0.025} & 0.004 & 1 & 18 & 4 \\
\hline & & 0.008 & 4 & 16 & 5 \\
\hline & & 0.012 & 2 & 15 & 2 \\
\hline & \multirow{3}{*}{0.05} & 0.004 & 12 & 10 & 11 \\
\hline & & 0.008 & 8 & 11 & 8 \\
\hline & & 0.012 & 11 & 7 & 9 \\
\hline & \multirow{3}{*}{0.1} & 0.004 & 16 & 4 & 13 \\
\hline & & 0.008 & 15 & 5 & 17 \\
\hline & & 0.012 & 18 & 2 & 14 \\
\hline \multirow{9}{*}{$\mathrm{UCB}$} & \multirow{3}{*}{0.025} & 0.004 & 5 & 13 & 1 \\
\hline & & 0.008 & 3 & 17 & 6 \\
\hline & & 0.012 & 6 & 14 & 3 \\
\hline & \multirow{3}{*}{0.05} & 0.004 & 9 & 12 & 12 \\
\hline & & 0.008 & 7 & 8 & 10 \\
\hline & & 0.012 & 10 & 9 & 7 \\
\hline & \multirow{3}{*}{0.1} & 0.004 & 13 & 3 & 16 \\
\hline & & 0.008 & 14 & 6 & 15 \\
\hline & & 0.012 & 17 & 1 & 18 \\
\hline
\end{tabular}

NOTE: No data for Flat insert due to tool breakage. 


\section{APPENDIX D}

\section{MEASURED VALUES OF CHIP SIDE FLOW ANGLE}

(this page intentionally left blank) 
Table D.1: Measured values for chip side flow angle, $\eta_{\mathrm{c}}$. Work material: AISI 1018, $\psi_{r}=\mathbf{4 5}^{\mathbf{0}}$.

Material: AISI 1018 Steel

Cutting Speed (V): $110 \mathrm{sfm}$

Lead Angle ( $\psi$ ): $45^{\circ}$

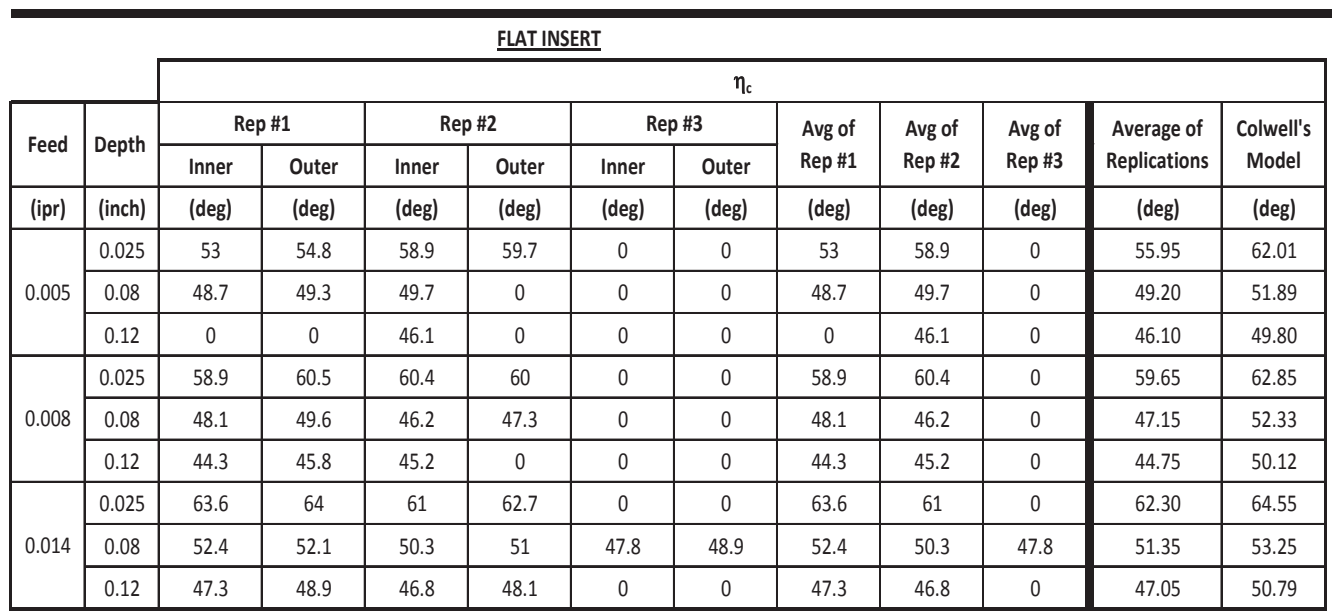

SANDVIK

\begin{tabular}{|c|c|c|c|c|c|c|c|c|c|c|c|c|}
\hline & \multirow{3}{*}{ Depth } & \multicolumn{11}{|c|}{$\eta_{c}$} \\
\hline \multirow{2}{*}{ Feed } & & \multicolumn{2}{|c|}{ Rep \#1 } & \multicolumn{2}{|c|}{ Rep \#2 } & \multicolumn{2}{|c|}{ Rep \#3 } & \multirow{2}{*}{$\begin{array}{l}\text { Avg of } \\
\text { Rep \#1 }\end{array}$} & \multirow{2}{*}{$\begin{array}{l}\text { Avg of } \\
\text { Rep \#2 }\end{array}$} & \multirow{2}{*}{$\begin{array}{l}\text { Avg of } \\
\text { Rep \#3 }\end{array}$} & \multirow{2}{*}{$\begin{array}{l}\text { Average of } \\
\text { Replications }\end{array}$} & \multirow{2}{*}{$\begin{array}{l}\text { Colwell's } \\
\text { Model }\end{array}$} \\
\hline & & Inner & Outer & Inner & Outer & Inner & Outer & & & & & \\
\hline (ipr) & (inch) & (deg) & (deg) & (deg) & (deg) & (deg) & (deg) & (deg) & (deg) & (deg) & (deg) & (deg) \\
\hline \multirow{3}{*}{0.005} & 0.025 & 51 & 0 & 56.2 & 57.4 & 53.4 & 55.2 & 51 & 56.2 & 53.4 & 53.53 & 62.01 \\
\hline & 0.08 & 50.3 & 0 & 47.2 & 0 & 48.2 & 0 & 50.3 & 47.2 & 48.2 & 48.57 & 51.89 \\
\hline & 0.12 & 45.9 & 0 & 48.1 & 0 & 50.7 & 52.4 & 45.9 & 48.1 & 50.7 & 48.23 & 49.80 \\
\hline \multirow{3}{*}{0.008} & 0.025 & 54 & 0 & 59.1 & 61 & 60.6 & 62.1 & 54 & 59.1 & 60.6 & 57.90 & 62.85 \\
\hline & 0.08 & 52.6 & 0 & 49.3 & 0 & 52.1 & 0 & 52.6 & 49.3 & 52.1 & 51.33 & 52.33 \\
\hline & 0.12 & 47.9 & 0 & 47.1 & 0 & 49.6 & 0 & 47.9 & 47.1 & 49.6 & 48.20 & 50.12 \\
\hline \multirow{3}{*}{0.014} & 0.025 & 56 & 0 & 57.4 & 0 & 56.4 & 0 & 56 & 57.4 & 56.4 & 56.60 & 64.55 \\
\hline & 0.08 & 56.7 & 0 & 56.3 & 0 & 55.9 & 56.8 & 56.7 & 56.3 & 55.9 & 56.30 & 53.25 \\
\hline & 0.12 & 51.2 & 51.4 & 50.7 & 0 & 50.1 & 51.2 & 51.2 & 50.7 & 50.1 & 50.67 & 50.79 \\
\hline
\end{tabular}

UNIVERSAL CHIP BREAKER

\begin{tabular}{|c|c|c|c|c|c|c|c|c|c|c|c|c|c|}
\hline \multirow{3}{*}{ Feed } & \multirow{3}{*}{ Depth } & \multicolumn{12}{|c|}{$\eta_{c}$} \\
\hline & & \multicolumn{2}{|c|}{ Rep \#1 } & \multicolumn{2}{|c|}{ Rep \#2 } & \multicolumn{2}{|c|}{ Rep \#3 } & \multirow{2}{*}{$\begin{array}{l}\text { Avg of } \\
\text { Rep \#1 }\end{array}$} & \multirow{2}{*}{$\begin{array}{l}\text { Avg of } \\
\text { Rep \#2 }\end{array}$} & \multirow{2}{*}{$\begin{array}{l}\text { Avg of } \\
\text { Rep \#3 }\end{array}$} & \multirow{2}{*}{$\begin{array}{l}\text { Average of } \\
\text { Replications }\end{array}$} & \multirow{2}{*}{$\begin{array}{l}\text { Colwell's } \\
\text { Model }\end{array}$} & \multirow{2}{*}{$\begin{array}{c}\text { Theoretically } \\
\text { Required Side } \\
\text { Flow Angle }\end{array}$} \\
\hline & & Inner & Outer & Inner & Outer & Inner & Outer & & & & & & \\
\hline (ipr) & (inch) & (deg) & (deg) & (deg) & (deg) & (deg) & (deg) & (deg) & (deg) & (deg) & (deg) & (deg) & (deg) \\
\hline \multirow{3}{*}{0.005} & 0.025 & 72 & 74 & 69.5 & 71.2 & 68.6 & 69.1 & 73 & 70.35 & 68.85 & 70.73 & 62.01 & 86.27 \\
\hline & 0.08 & 61.3 & 61 & 53.2 & 53.4 & 56.1 & 57.3 & 61.15 & 53.3 & 56.7 & 57.05 & 51.89 & 80.64 \\
\hline & 0.12 & 56.1 & 57.4 & 55.2 & 57.1 & 54.4 & 57.9 & 56.75 & 56.15 & 56.15 & 56.35 & 49.80 & 75.98 \\
\hline \multirow{3}{*}{0.008} & 0.025 & 71 & 71.2 & 67.4 & 70.3 & 59 & 60.7 & 71.1 & 68.85 & 59.85 & 66.60 & 62.85 & 86.40 \\
\hline & 0.08 & 56.6 & 56 & 57.7 & 58 & 58.6 & 62.3 & 56.3 & 57.85 & 60.45 & 58.20 & 52.33 & 80.78 \\
\hline & 0.12 & 48 & 51.1 & 52.9 & 53.3 & 50.2 & 53.1 & 49.55 & 53.1 & 51.65 & 51.43 & 50.12 & 76.12 \\
\hline \multirow{3}{*}{0.014} & \begin{tabular}{|l}
0.025 \\
\end{tabular} & 78 & 79 & 79.2 & 79 & 81.1 & 85 & 78.5 & 79.1 & 83.05 & 80.22 & 64.55 & 86.67 \\
\hline & 0.08 & 60.2 & 63.3 & 59.1 & 60 & 56 & 65 & 61.75 & 59.55 & 60.5 & 60.60 & 53.25 & 81.06 \\
\hline & 0.12 & 56.7 & 58.5 & 57.8 & 60.2 & 58.7 & 64 & 57.6 & 59 & 61.35 & 59.32 & 50.79 & 76.41 \\
\hline
\end{tabular}


Table D.2: Measured values for chip side flow angle, $\eta_{\mathrm{c}}$. Work material: AISI 1018, $\psi_{r}=\mathbf{1 5}^{\mathbf{0}}$.

Material: $\quad$ AISI 1018 Steel

Cutting Speed (V): $110 \mathrm{sfm}$

Lead Angle ( $\psi): 15^{\circ}$

\begin{tabular}{|c|c|c|c|c|c|c|c|c|c|c|c|c|}
\hline \multicolumn{13}{|c|}{ FLAT INSERT } \\
\hline & & & & & & & & $c$ & & & & \\
\hline \multirow[b]{2}{*}{ Feed } & \multirow[b]{2}{*}{ Depth } & \multicolumn{2}{|c|}{ Rep \#1 } & \multicolumn{2}{|c|}{ Rep \#2 } & \multicolumn{2}{|c|}{ Rep \#3 } & \multirow{2}{*}{$\begin{array}{l}\text { Avg of } \\
\text { Rep \#1 }\end{array}$} & \multirow{2}{*}{$\begin{array}{l}\text { Avg of } \\
\text { Rep \#2 }\end{array}$} & \multirow{2}{*}{$\begin{array}{l}\text { Avg of } \\
\text { Rep \#3 }\end{array}$} & \multirow{2}{*}{$\begin{array}{l}\text { Average of } \\
\text { Replications }\end{array}$} & \multirow{2}{*}{ Colwell's Model } \\
\hline & & Inner & Outer & Inner & Outer & Inner & Outer & & & & & \\
\hline (ipr) & (inch) & (deg) & (deg) & (deg) & (deg) & (deg) & (deg) & (deg) & (deg) & (deg) & (deg) & (deg) \\
\hline \multirow{3}{*}{0.005} & 0.025 & 58.1 & 58.7 & 60.1 & 60 & 0 & 0 & 58.1 & 60.1 & 0 & 59.10 & 60.44 \\
\hline & 0.08 & 46.7 & 46 & 0 & 0 & 0 & 0 & 46.7 & 0 & 0 & 46.70 & 36.85 \\
\hline & 0.12 & 32.1 & 0 & 0 & 0 & 0 & 0 & 32.1 & 0 & 0 & 32.10 & 30.49 \\
\hline \multirow{3}{*}{0.008} & 0.025 & 63.1 & 64 & 65.4 & 67 & 0 & 0 & 63.1 & 65.4 & 0 & 64.25 & 61.36 \\
\hline & 0.08 & 30.4 & 30 & 28.7 & 28.5 & 0 & 0 & 30.4 & 28.7 & 0 & 29.55 & 37.57 \\
\hline & 0.12 & 0 & 0 & 0 & 0 & 0 & 0 & 0 & 0 & 0 & 0.00 & 31.04 \\
\hline \multirow{3}{*}{0.014} & 0.025 & 65.2 & 67 & 59.3 & 63.1 & 0 & 0 & 65.2 & 59.3 & 0 & 62.25 & 63.20 \\
\hline & 0.08 & 28.9 & 29.4 & 33.4 & 33.2 & 0 & 0 & 28.9 & 33.4 & 0 & 31.15 & 39.02 \\
\hline & 0.12 & 26.1 & 26 & 0 & 0 & 0 & 0 & 26.1 & 0 & 0 & 26.10 & 32.16 \\
\hline
\end{tabular}

SANDVIK

\begin{tabular}{|c|c|c|c|c|c|c|c|c|c|c|c|c|}
\hline \multirow{3}{*}{ Feed } & & \multicolumn{11}{|c|}{$\eta_{c}$} \\
\hline & \multirow{2}{*}{ Depth } & \multicolumn{2}{|c|}{ Rep \#1 } & \multicolumn{2}{|c|}{ Rep \#2 } & \multicolumn{2}{|c|}{ Rep \#3 } & \multirow{2}{*}{$\begin{array}{l}\text { Avg of } \\
\text { Rep \#1 }\end{array}$} & \multirow{2}{*}{$\begin{array}{l}\text { Avg of } \\
\text { Rep \#2 }\end{array}$} & \multirow{2}{*}{$\begin{array}{l}\text { Avg of } \\
\text { Rep \#3 }\end{array}$} & \multirow{2}{*}{$\begin{array}{l}\text { Average of } \\
\text { Replications }\end{array}$} & \multirow{2}{*}{ Colwell's Mode } \\
\hline & & Inner & Outer & Inner & Outer & Inner & Outer & & & & & \\
\hline (ipr) & (inch) & (deg) & (deg) & (deg) & (deg) & (deg) & (deg) & (deg) & (deg) & (deg) & (deg) & (deg) \\
\hline \multirow{3}{*}{0.005} & 0.025 & 57 & 59.3 & 59.2 & 59 & 62 & 62.2 & 57 & 59.2 & 62 & 59.40 & 60.44 \\
\hline & 0.08 & 26.2 & 0 & 32.1 & 0 & 28.2 & 0 & 26.2 & 32.1 & 28.2 & 28.83 & 36.85 \\
\hline & 0.12 & 28.4 & 0 & 28.9 & 0 & 26.9 & 0 & 28.4 & 28.9 & 26.9 & 28.07 & 30.49 \\
\hline \multirow{3}{*}{0.008} & 0.025 & 58.6 & 59.1 & 61.3 & 63.8 & 61.4 & 64.1 & 58.6 & 61.3 & 61.4 & 60.43 & 61.36 \\
\hline & 0.08 & 25.9 & 26.1 & 30.8 & 32.1 & 31.4 & 32.4 & 25.9 & 30.8 & 31.4 & 29.37 & 37.57 \\
\hline & 0.12 & 30.1 & 0 & 29.1 & 30 & 28.1 & 29 & 30.1 & 29.1 & 28.1 & 29.10 & 31.04 \\
\hline \multirow{3}{*}{0.014} & 0.025 & 42.7 & 0 & 61.1 & 63.9 & 62.1 & 63.7 & 42.7 & 61.1 & 62.1 & 55.30 & 63.20 \\
\hline & 0.08 & 29.1 & 30 & 30.1 & 32 & 30 & 30.1 & 29.1 & 30.1 & 30 & 29.73 & 39.02 \\
\hline & 0.12 & 24.7 & 25.8 & 31.4 & 33.2 & 26.4 & 27.4 & 24.7 & 31.4 & 26.4 & 27.50 & 32.16 \\
\hline
\end{tabular}

UNIVERSAL CHIP BREAKER

\begin{tabular}{|c|c|c|c|c|c|c|c|c|c|c|c|c|c|}
\hline \multirow[b]{3}{*}{ Feed } & \multirow[b]{3}{*}{ Depth } & \multicolumn{12}{|c|}{$\eta_{\mathrm{c}}$} \\
\hline & & \multicolumn{2}{|c|}{ Rep \#1 } & \multicolumn{2}{|c|}{ Rep \#2 } & \multicolumn{2}{|c|}{ Rep \#3 } & \multirow{2}{*}{$\begin{array}{l}\text { Avg of } \\
\text { Rep \#1 }\end{array}$} & \multirow{2}{*}{$\begin{array}{l}\text { Avg of } \\
\text { Rep \#2 }\end{array}$} & \multirow{2}{*}{$\begin{array}{l}\text { Avg of } \\
\text { Rep \#3 }\end{array}$} & \multirow{2}{*}{$\begin{array}{l}\text { Average of } \\
\text { Replications }\end{array}$} & \multirow[b]{2}{*}{ Colwell's Model } & \multirow{2}{*}{$\begin{array}{c}\text { Theoretically } \\
\text { Required Side Flow } \\
\text { Angle }\end{array}$} \\
\hline & & Inner & Outer & Inner & Outer & Inner & Outer & & & & & & \\
\hline (ipr) & (inch) & (deg) & (deg) & (deg) & (deg) & (deg) & (deg) & (deg) & (deg) & (deg) & (deg) & (deg) & (deg) \\
\hline \multirow{2}{*}{0.005} & 0.025 & 44.3 & 45.1 & 45.2 & 46 & 45.7 & 46.1 & 44.7 & 45.6 & 45.9 & 45.40 & 60.44 & 58.55 \\
\hline & 0.12 & 24.2 & 25.7 & 25.9 & 25.7 & 24.6 & 24.4 & 24.95 & 25.8 & 24.5 & 25.08 & 30.49 & 52.62 \\
\hline \multirow{3}{*}{0.008} & 0.025 & 43.8 & 44.6 & 48.5 & 49 & 43.3 & 45.7 & 44.2 & 48.75 & 44.5 & 45.82 & 61.36 & 58.46 \\
\hline & 0.08 & 26.1 & 26.4 & 34.1 & 34 & 26.3 & 26.9 & 26.25 & 34.05 & 26.6 & 28.97 & 37.57 & 54.88 \\
\hline & 0.12 & 27.3 & 28 & 27.6 & 28.3 & 26.2 & 27.2 & 27.65 & 27.95 & 26.7 & 27.43 & 31.04 & 52.53 \\
\hline 0.014 & 0.025 & 49.6 & 49.2 & 50.2 & 51.9 & 50 & 49.8 & 49.4 & 51.05 & 49.9 & 50.12 & 63.20 & 58.28 \\
\hline
\end{tabular}


Table D.3: Measured values for chip side flow angle, $\eta_{\mathrm{c}}$. Work material: AL 2024, $\psi_{r}=4^{\circ}$.

Material: AL 2024

Cutting Speed (V): $225 \mathrm{sfm}$

Lead Angle $(\psi): 45^{\circ}$

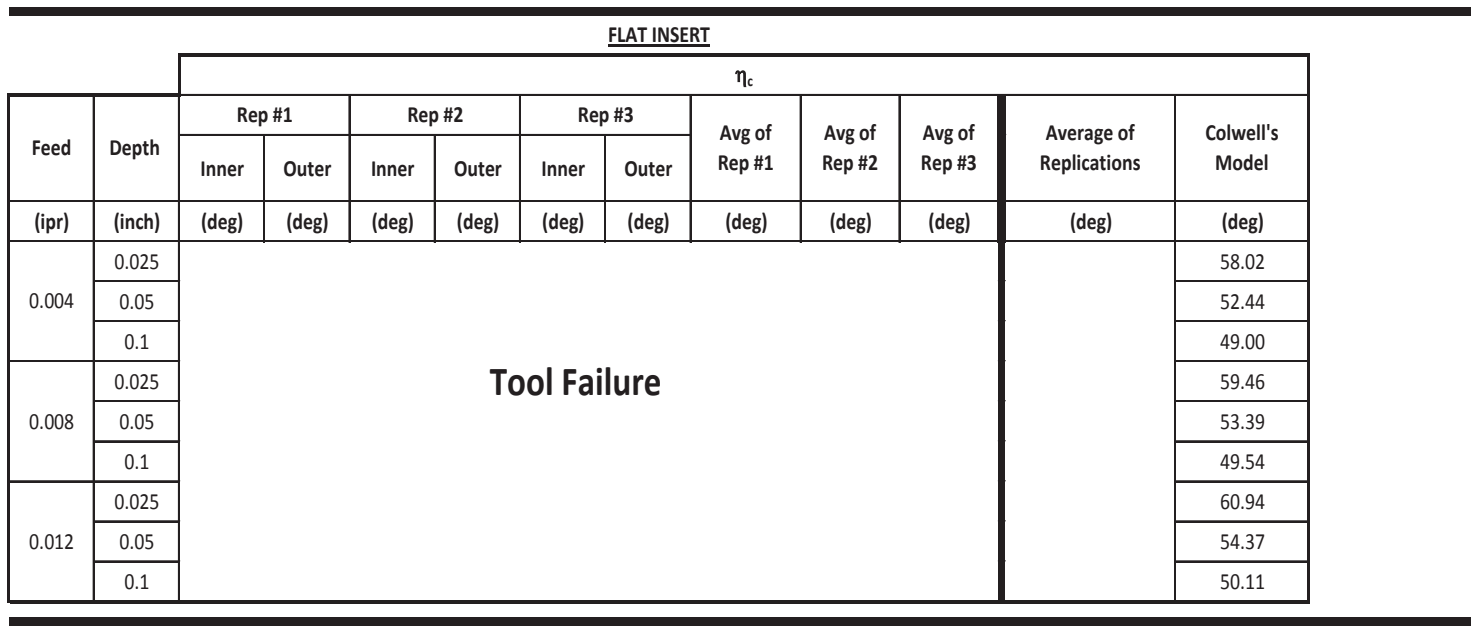

Korloy

\begin{tabular}{|c|c|c|c|c|c|c|c|c|c|c|c|c|}
\hline & & & & & & & & $\eta_{\mathrm{c}}$ & & & & \\
\hline \multirow{2}{*}{ Feed } & \multirow{2}{*}{ Depth } & \multicolumn{2}{|c|}{ Rep \#1 } & \multicolumn{2}{|c|}{ Rep \#2 } & \multicolumn{2}{|c|}{ Rep \#3 } & \multirow{2}{*}{$\begin{array}{l}\text { Avg of } \\
\text { Rep \#1 }\end{array}$} & \multirow{2}{*}{$\begin{array}{l}\text { Avg of } \\
\text { Rep \#2 }\end{array}$} & \multirow{2}{*}{$\begin{array}{l}\text { Avg of } \\
\text { Rep \#3 }\end{array}$} & \multirow{2}{*}{$\begin{array}{l}\text { Average of } \\
\text { Replications }\end{array}$} & \multirow{2}{*}{$\begin{array}{c}\text { Colwell's } \\
\text { Model }\end{array}$} \\
\hline & & Inner & Outer & Inner & Outer & Inner & Outer & & & & & \\
\hline (ipr) & (inch) & (deg) & (deg) & (deg) & (deg) & (deg) & (deg) & (deg) & (deg) & (deg) & (deg) & (deg) \\
\hline \multirow{3}{*}{0.004} & 0.025 & 68.7 & 68.5 & 58.4 & 0 & 71.3 & 71 & 68.7 & 58.4 & 71.3 & 66.13 & 58.02 \\
\hline & 0.05 & 47.1 & 0 & 58 & 0 & 51.3 & 52 & 47.1 & 58 & 51.3 & 52.13 & 52.44 \\
\hline & 0.1 & 46.3 & 0 & 52.6 & 0 & 45.1 & 0 & 46.3 & 52.6 & 45.1 & 48.00 & 49.00 \\
\hline \multirow{3}{*}{0.008} & 0.025 & 71.2 & 71.1 & 70 & 70.6 & 70.2 & 0 & 71.2 & 70 & 70.2 & 70.47 & 59.46 \\
\hline & 0.05 & 53 & 53.2 & 58.4 & 0 & 50.9 & 51 & 53 & 58.4 & 50.9 & 54.10 & 53.39 \\
\hline & 0.1 & 47.1 & 0 & 53.8 & 0 & 46.3 & 0 & 47.1 & 53.8 & 46.3 & 49.07 & 49.54 \\
\hline \multirow{3}{*}{0.012} & 0.025 & 53.1 & 0 & 57.3 & 57.5 & 72.1 & 0 & 53.1 & 57.3 & 72.1 & 60.83 & 60.94 \\
\hline & 0.05 & 55.7 & 0 & 57.9 & 0 & 51 & 0 & 55.7 & 57.9 & 51 & 54.87 & 54.37 \\
\hline & 0.1 & 54.3 & 0 & 54.1 & 0 & 47 & 0 & 54.3 & 54.1 & 47 & 51.80 & 50.11 \\
\hline
\end{tabular}

UNIVERSAL CHIP BREAKER

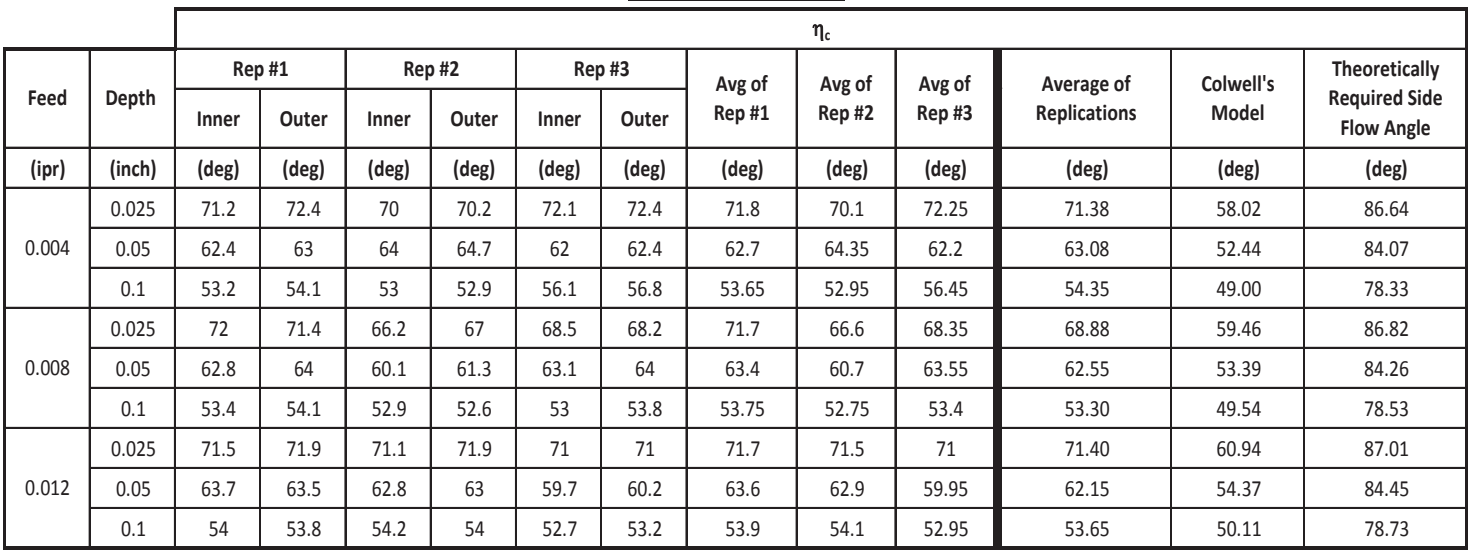


Table D.4: Measured values for chip side flow angle, $\eta_{\mathrm{c}}$. Work material: AL 2024, $\psi_{r}=\mathbf{1 5}^{\mathbf{0}}$.

Material: AL 2024

Cutting Speed (V): $225 \mathrm{sfm}$

Lead Angle $(\psi): 15^{\circ}$

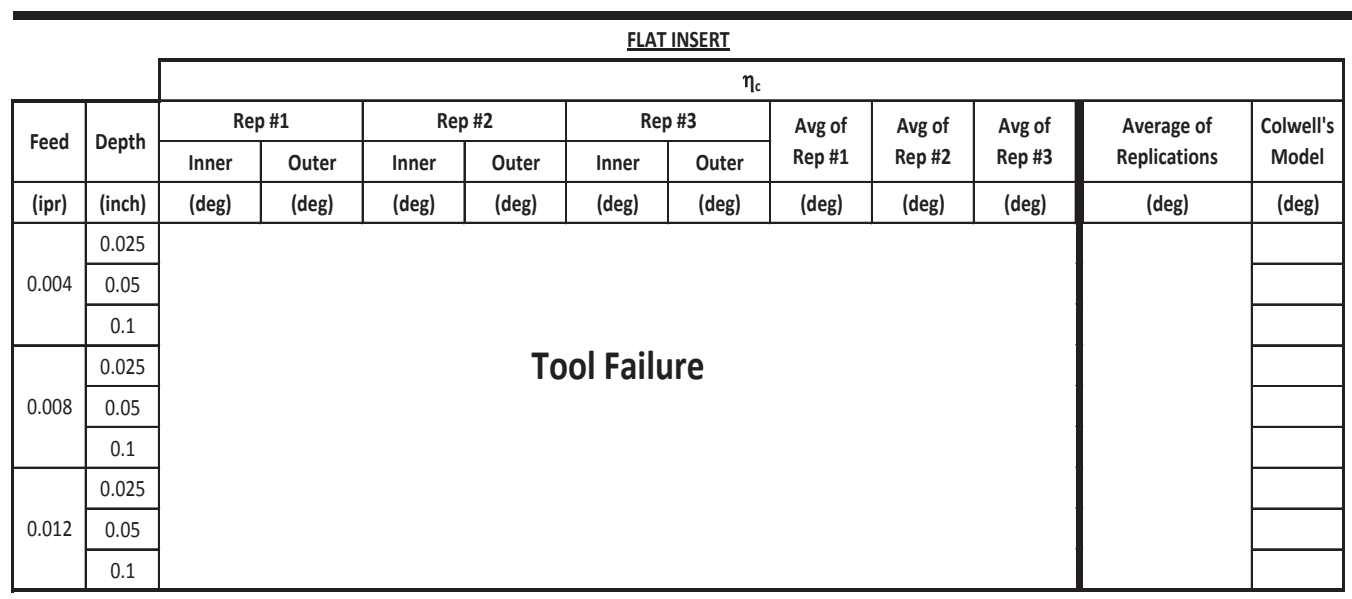

\begin{tabular}{|c|c|c|c|c|c|c|c|c|c|c|c|c|}
\hline \multirow{4}{*}{ Feed } & \multirow{4}{*}{ Depth } & \multicolumn{11}{|c|}{ Korloy } \\
\hline & & \multicolumn{11}{|c|}{$\eta_{c}$} \\
\hline & & \multicolumn{2}{|c|}{ Rep \#1 } & \multicolumn{2}{|c|}{ Rep \#2 } & \multicolumn{2}{|c|}{ Rep \#3 } & \multirow{2}{*}{$\begin{array}{l}\text { Avg of } \\
\text { Rep \#1 }\end{array}$} & \multirow{2}{*}{$\begin{array}{l}\text { Avg of } \\
\text { Rep \#2 }\end{array}$} & \multirow{2}{*}{$\begin{array}{l}\text { Avg of } \\
\text { Rep \#3 }\end{array}$} & \multirow{2}{*}{$\begin{array}{l}\text { Average of } \\
\text { Replications }\end{array}$} & \multirow{2}{*}{$\begin{array}{c}\text { Colwell's } \\
\text { Model }\end{array}$} \\
\hline & & Inner & Outer & Inner & Outer & Inner & Outer & & & & & \\
\hline (ipr) & (inch) & (deg) & (deg) & (deg) & (deg) & (deg) & (deg) & (deg) & (deg) & (deg) & (deg) & (deg) \\
\hline \multirow{3}{*}{0.004} & 0.025 & 44.3 & 42.1 & 41.2 & 0 & 45.1 & 46.9 & 43.2 & 41.2 & 46 & 43.47 & 52.65 \\
\hline & 0.05 & 39 & 38 & 37.4 & 38.7 & 34.7 & 36.2 & 38.5 & 38.05 & 35.45 & 37.33 & 38.26 \\
\hline & 0.1 & 24.1 & 0 & 25.7 & 26.4 & 22.9 & 0 & 24.1 & 26.05 & 22.9 & 24.35 & 27.84 \\
\hline \multirow{3}{*}{0.008} & 0.025 & 54.2 & 55.4 & 52 & 56.1 & 52.2 & 53.8 & 54.8 & 54.05 & 53 & 53.95 & 54.49 \\
\hline & 0.05 & 36.5 & 37 & 36.6 & 37.5 & 32.7 & 34.1 & 36.75 & 37.05 & 33.4 & 35.73 & 39.75 \\
\hline & 0.1 & 28 & 0 & 29.1 & 0 & 23 & 0 & 28 & 29.1 & 23 & 26.70 & 28.77 \\
\hline \multirow{3}{*}{0.012} & 0.025 & 53.7 & 54.8 & 46.8 & 48 & 43.1 & 44.7 & 54.25 & 47.4 & 43.9 & 48.52 & 56.35 \\
\hline & 0.05 & 37.4 & 38.3 & 34.3 & 0 & 31.9 & 32.3 & 37.85 & 34.3 & 32.1 & 34.75 & 41.27 \\
\hline & 0.1 & 21.8 & 0 & 23.1 & 0 & 19 & 0 & 21.8 & 23.1 & 19 & 21.30 & 29.73 \\
\hline
\end{tabular}

\begin{tabular}{|c|c|c|c|c|c|c|c|c|c|c|c|c|c|}
\hline \multicolumn{14}{|c|}{ UNIVERSAL CHIP BREAKER } \\
\hline & & & & & & & & $\eta_{c}$ & & & & & \\
\hline \multirow{2}{*}{ Feed } & \multirow[b]{2}{*}{ Depth } & \multicolumn{2}{|c|}{ Rep \#1 } & \multicolumn{2}{|c|}{ Rep \#2 } & \multicolumn{2}{|c|}{ Rep \#3 } & \multirow{2}{*}{$\begin{array}{l}\text { Avg of } \\
\text { Rep \#1 }\end{array}$} & \multirow{2}{*}{$\begin{array}{l}\text { Avg of } \\
\text { Rep \#2 }\end{array}$} & \multirow{2}{*}{$\begin{array}{l}\text { Avg of } \\
\text { Rep \#3 }\end{array}$} & \multirow{2}{*}{$\begin{array}{l}\text { Average of } \\
\text { Replications }\end{array}$} & \multirow{2}{*}{$\begin{array}{c}\text { Colwell's } \\
\text { Model }\end{array}$} & \multirow{2}{*}{$\begin{array}{c}\text { Theoretically } \\
\text { Required Side } \\
\text { Flow Angle }\end{array}$} \\
\hline & & Inner & Outer & Inner & Outer & Inner & Outer & & & & & & \\
\hline (ipr) & (inch) & (deg) & (deg) & (deg) & (deg) & (deg) & (deg) & (deg) & (deg) & (deg) & (deg) & (deg) & (deg) \\
\hline \multirow{3}{*}{0.004} & 0.025 & 37 & 39.2 & 36.2 & 39 & 37.8 & 40.1 & 38.1 & 37.6 & 38.95 & 38.22 & 52.65 & 55.40 \\
\hline & 0.05 & 32.4 & 34.7 & 32 & 34 & 34 & 36.2 & 33.55 & 33 & 35.1 & 33.88 & 38.26 & 53.80 \\
\hline & 0.1 & 30.6 & 32.8 & 29.5 & 30 & 26.8 & 28 & 31.7 & 29.75 & 27.4 & 29.62 & 27.84 & 50.92 \\
\hline \multirow{3}{*}{0.008} & 0.025 & 36.2 & 37.5 & 37.6 & 38.1 & 34.2 & 35.4 & 36.85 & 37.85 & 34.8 & 36.50 & 54.49 & 55.27 \\
\hline & 0.05 & 34.8 & 36.7 & 32.8 & 33.6 & 32.6 & 33.9 & 35.75 & 33.2 & 33.25 & 34.07 & 39.75 & 53.68 \\
\hline & 0.1 & 29.1 & 30 & 27.6 & 28.2 & 27.1 & 29.2 & 29.55 & 27.9 & 28.15 & 28.53 & 28.77 & 50.79 \\
\hline \multirow{3}{*}{0.012} & 0.025 & 41 & 43.2 & 37.8 & 38 & 37.3 & 38.1 & 42.1 & 37.9 & 37.7 & 39.23 & 56.35 & 55.13 \\
\hline & 0.05 & 32 & 34.7 & 33 & 35.2 & 34.1 & 36.3 & 33.35 & 34.1 & 35.2 & 34.22 & 41.27 & 53.54 \\
\hline & 0.1 & 28.4 & 29.4 & 28.2 & 28 & 29.2 & 31.8 & 28.9 & 28.1 & 30.5 & 29.17 & 29.73 & 50.65 \\
\hline
\end{tabular}




\section{APPENDIX E}

\section{REPLICATED RESULTS OF CHIP FLOW TESTS}

(this page intentionally left blank) 


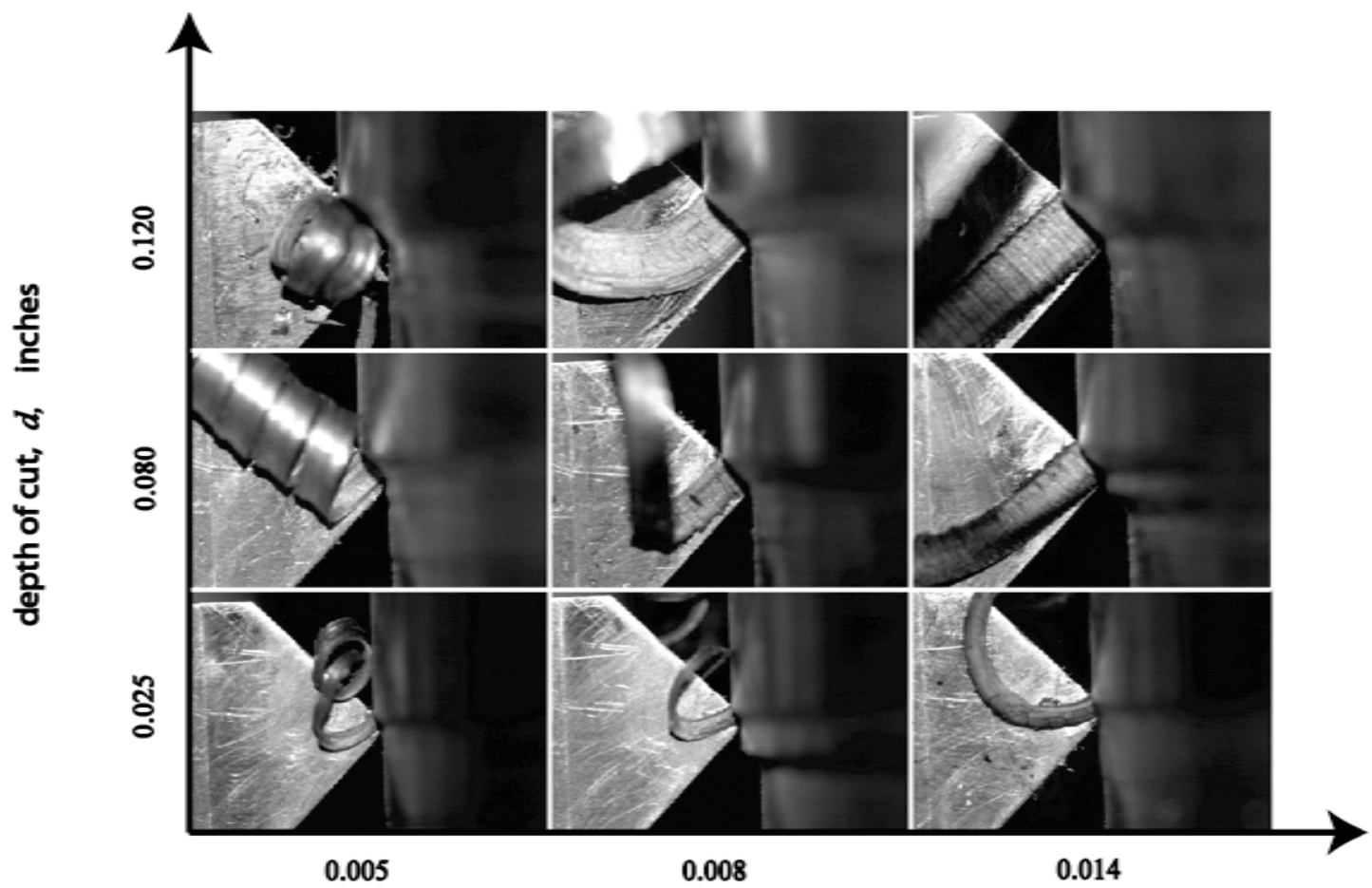

feed, $f$, ipr

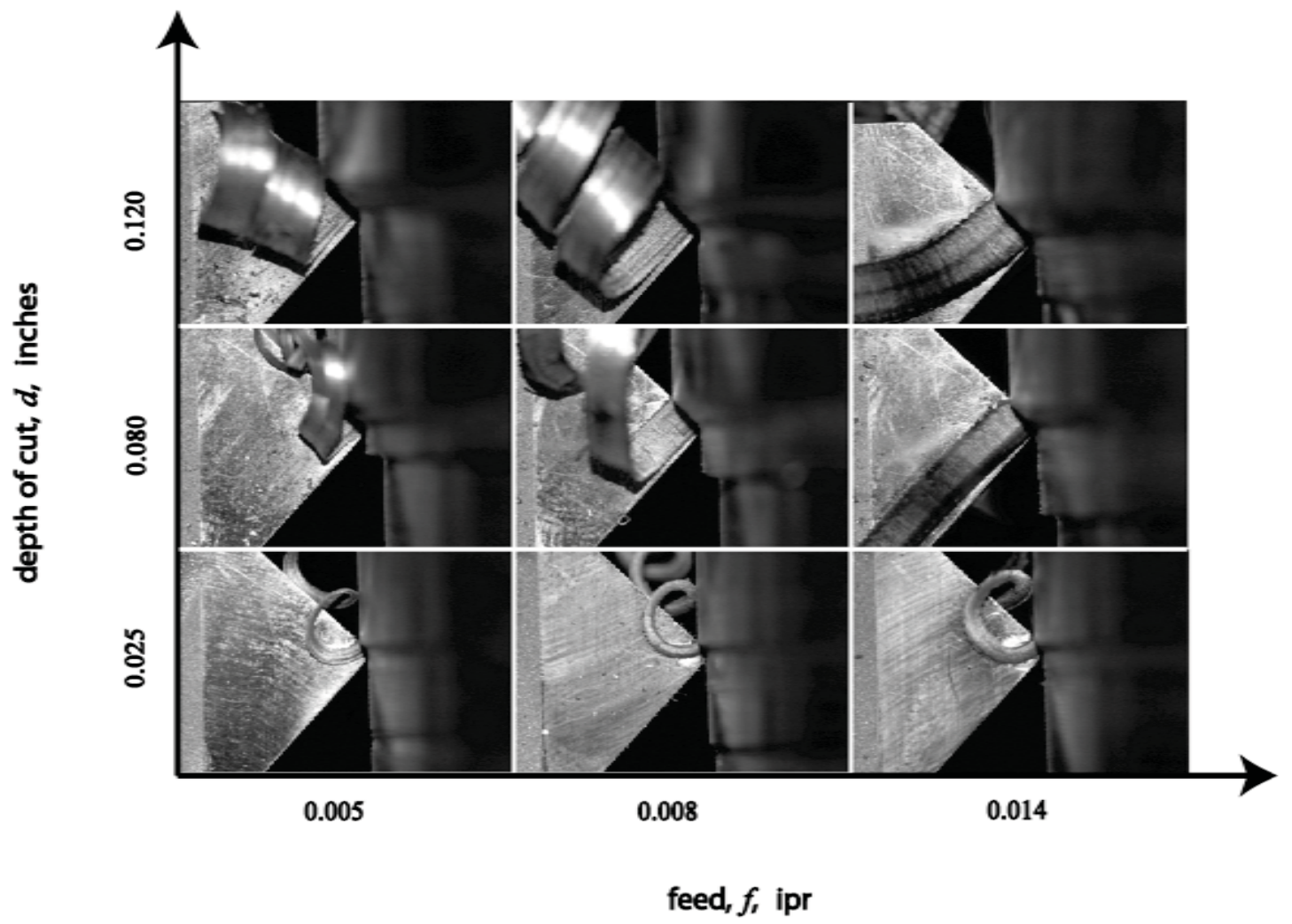




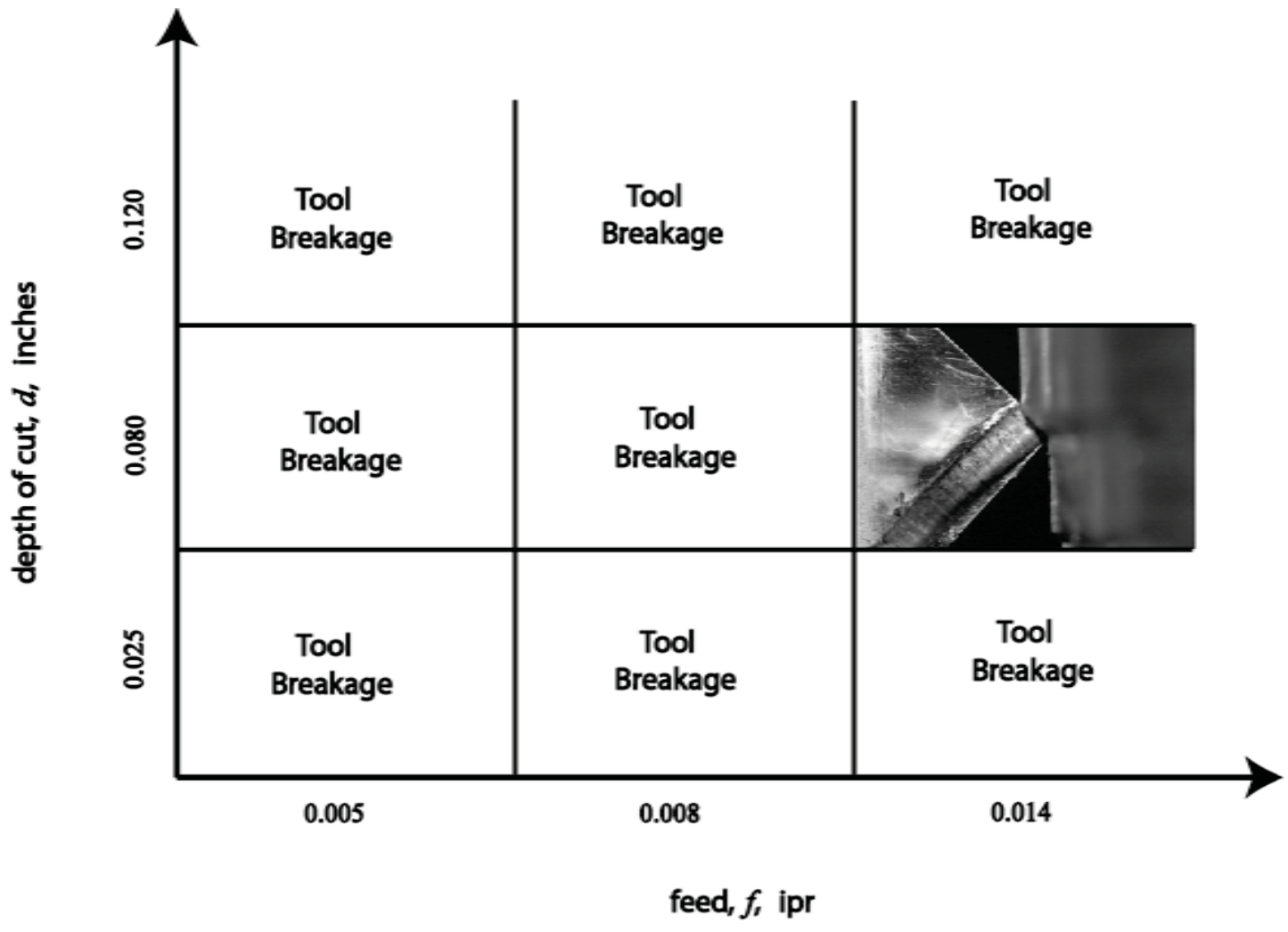

Figure E.1: Chip flow patterns for Flat tool (Previous page top) Replication \#1 (Previous page bottom) Replication \# 2 (This page top) Replication \#3; Work material: AISI 1018, $\psi_{r}=4^{\circ}$. 


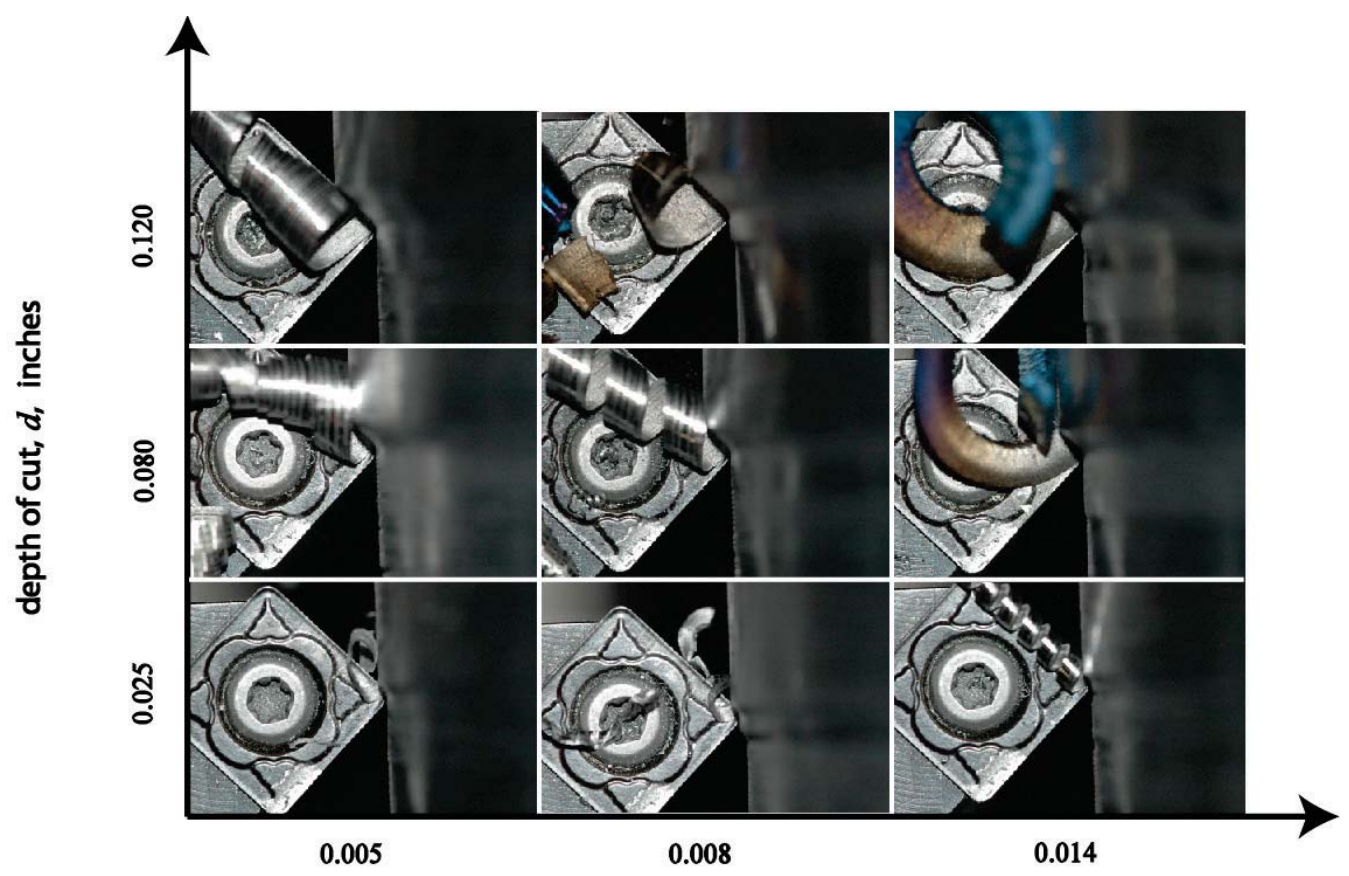

feed, $f$, ipr

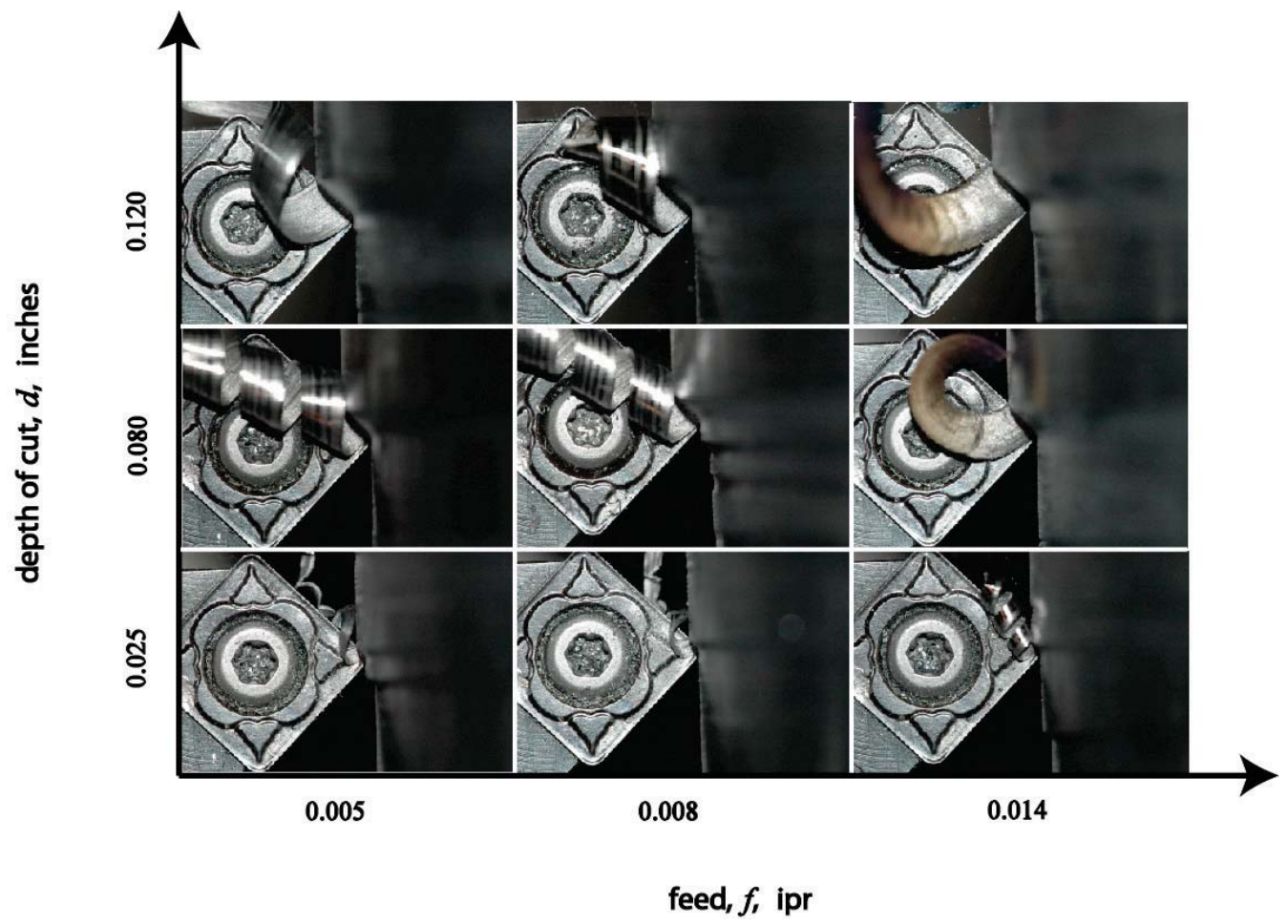

Figure E.2: Chip flow patterns for Sandvik S-433 insert (top) Replication \#2 (bottom) Replication \#3; Work material: AISI 1018, $\psi_{r}=\mathbf{4 5}^{\mathbf{0}}$. 


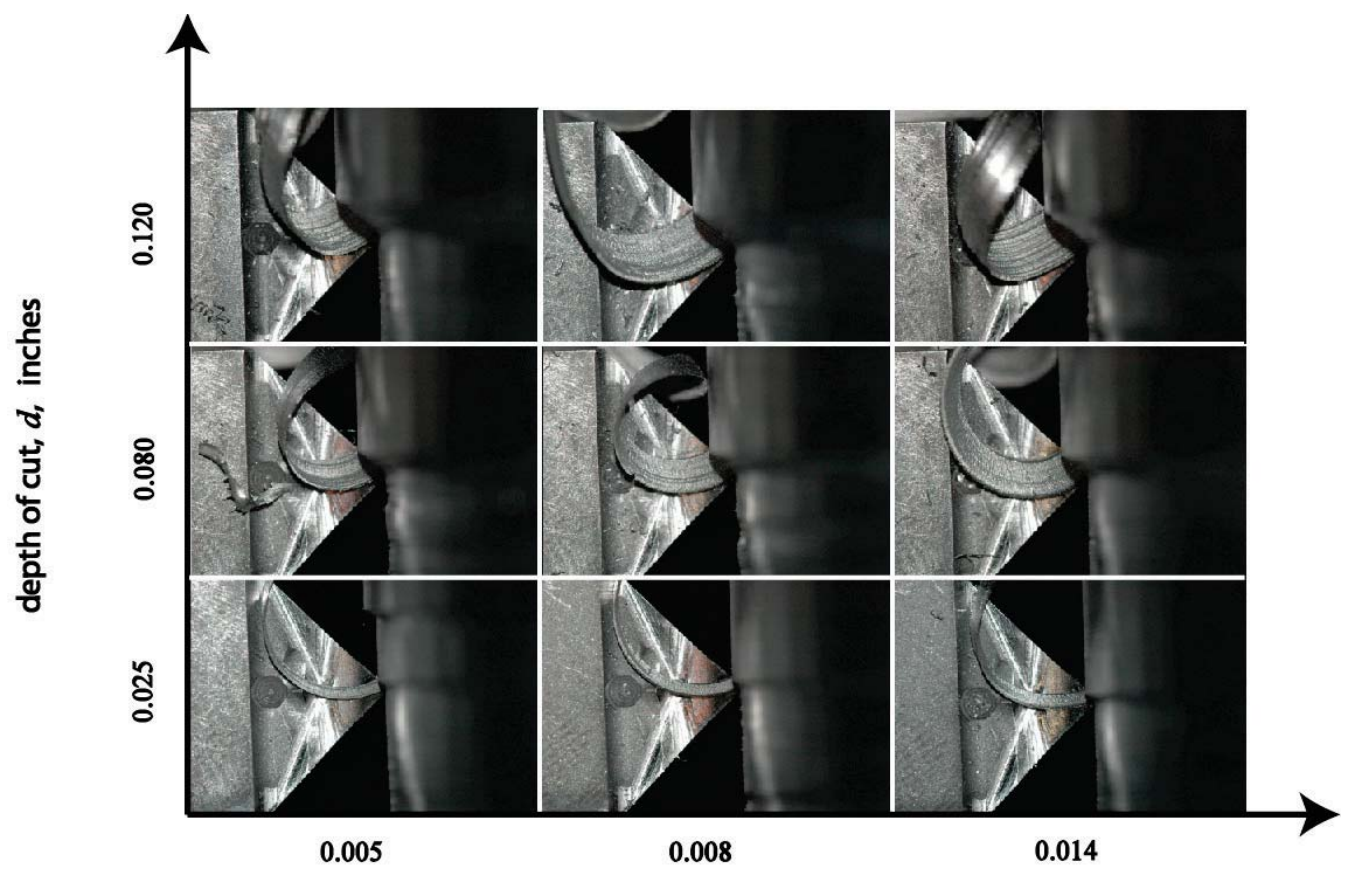

feed, $f$, ipr

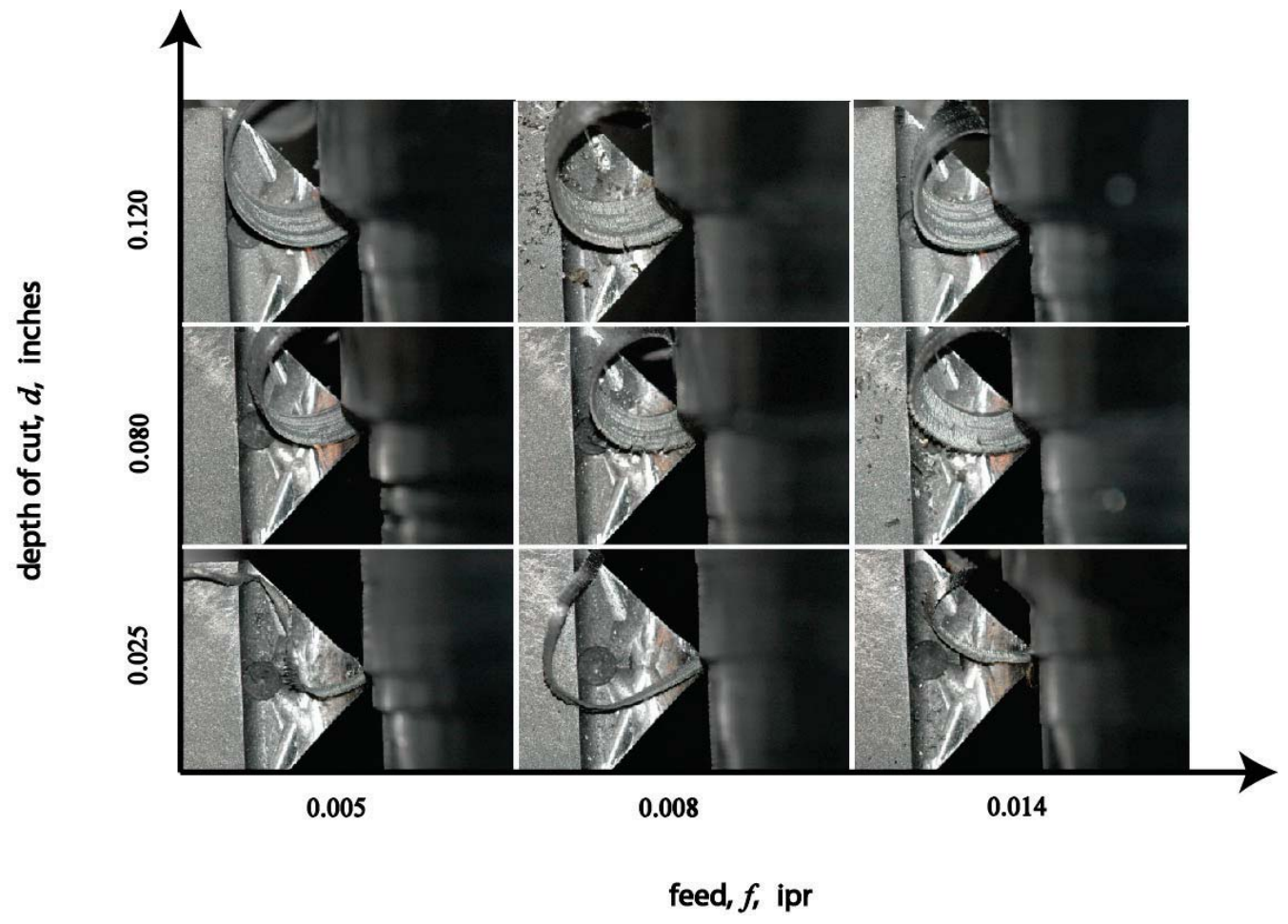

Figure E.3: Chip flow patterns for UCB tool (top) Replication \#2 (bottom) Replication \# 3; Work material: AISI 1018, $\psi_{r}=\mathbf{4 5}^{\circ}$. 


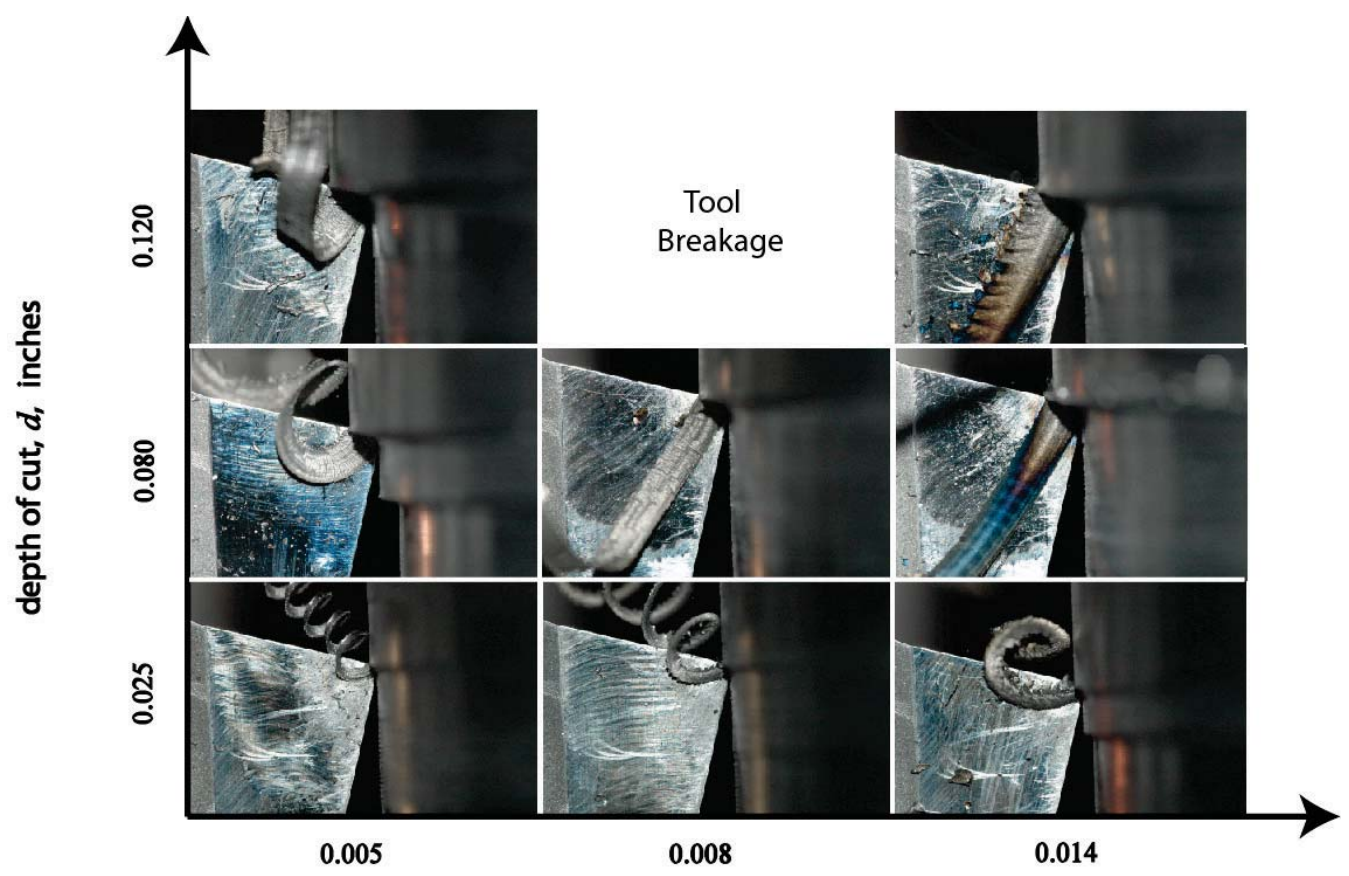

feed, $f$, ipr

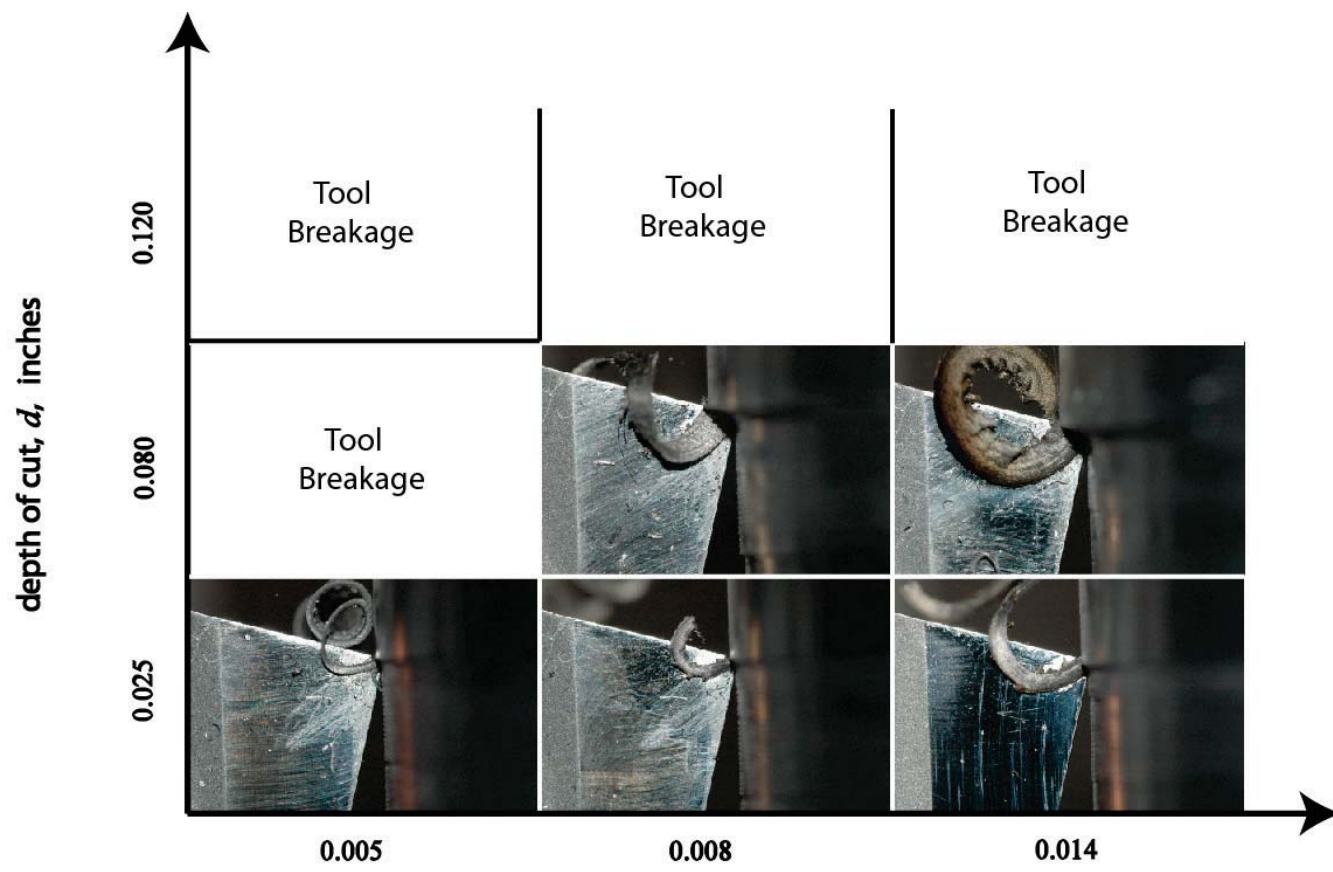

feed, $f$, ipr

Figure E.4: Chip flow patterns for Flat tool (top) Replication \#1 (bottom) Replication \#2; Work material: AISI 1018, $\psi_{r}=15^{\circ}$. NOTE: No data available for Replication \#3 due to tool failure. 


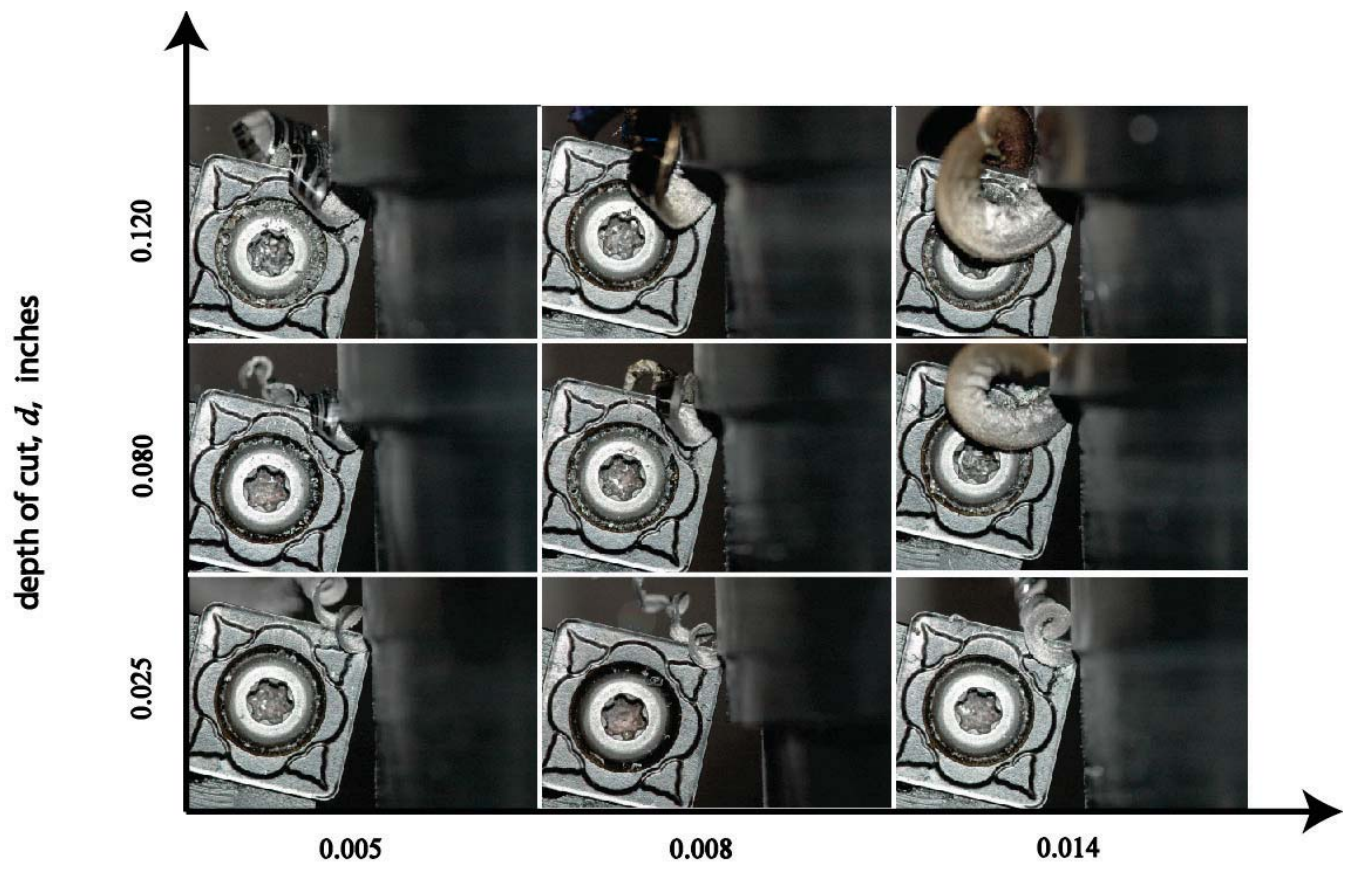

feed, $f$, ipr

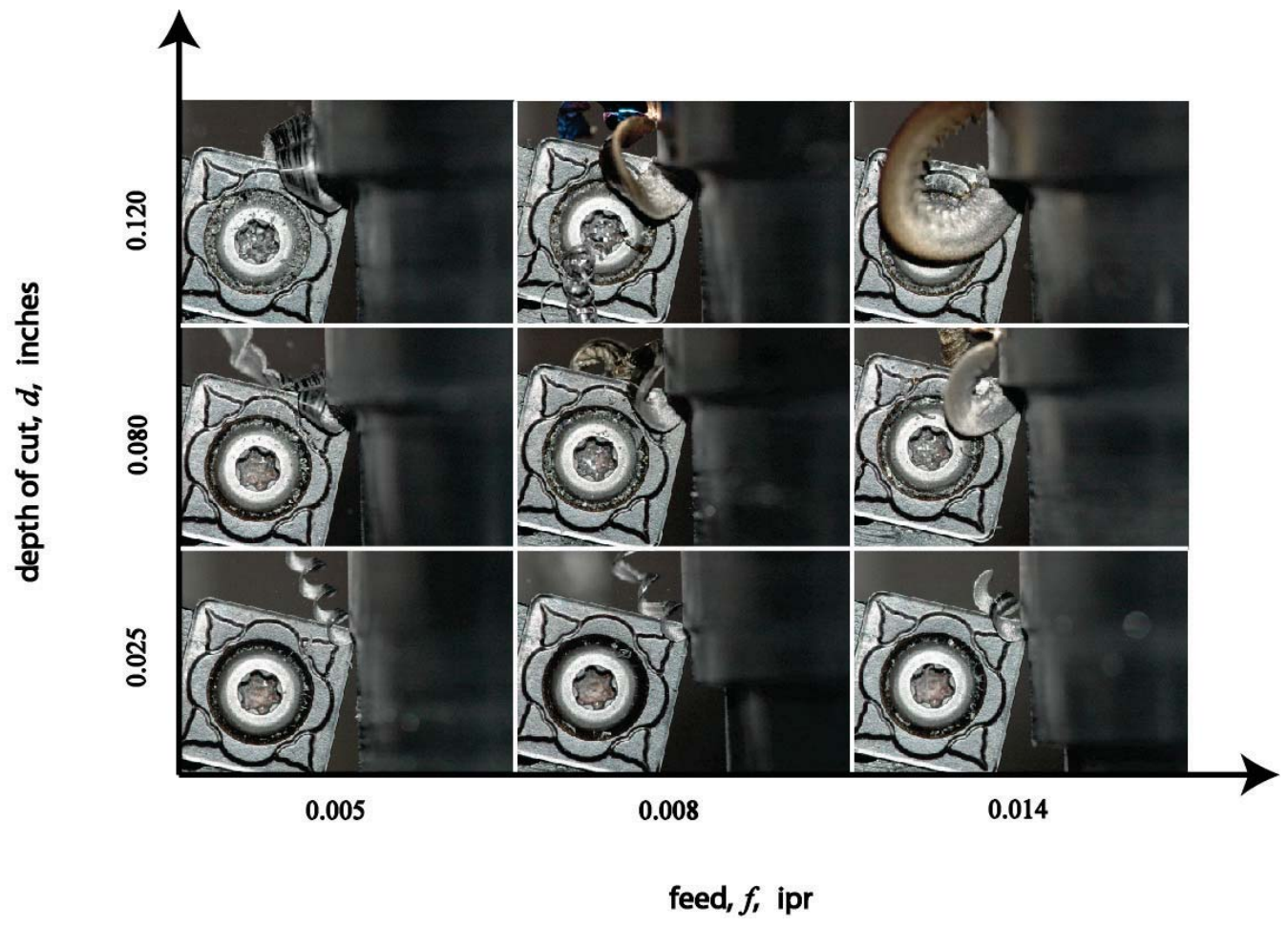

Figure E.5: Chip flow patterns for Sandvik S-433 insert (top) Replication \#2 (bottom) Replication \# 3; Work material: AISI 1018, $\psi_{r}=\mathbf{1 5}^{\mathbf{0}}$. 


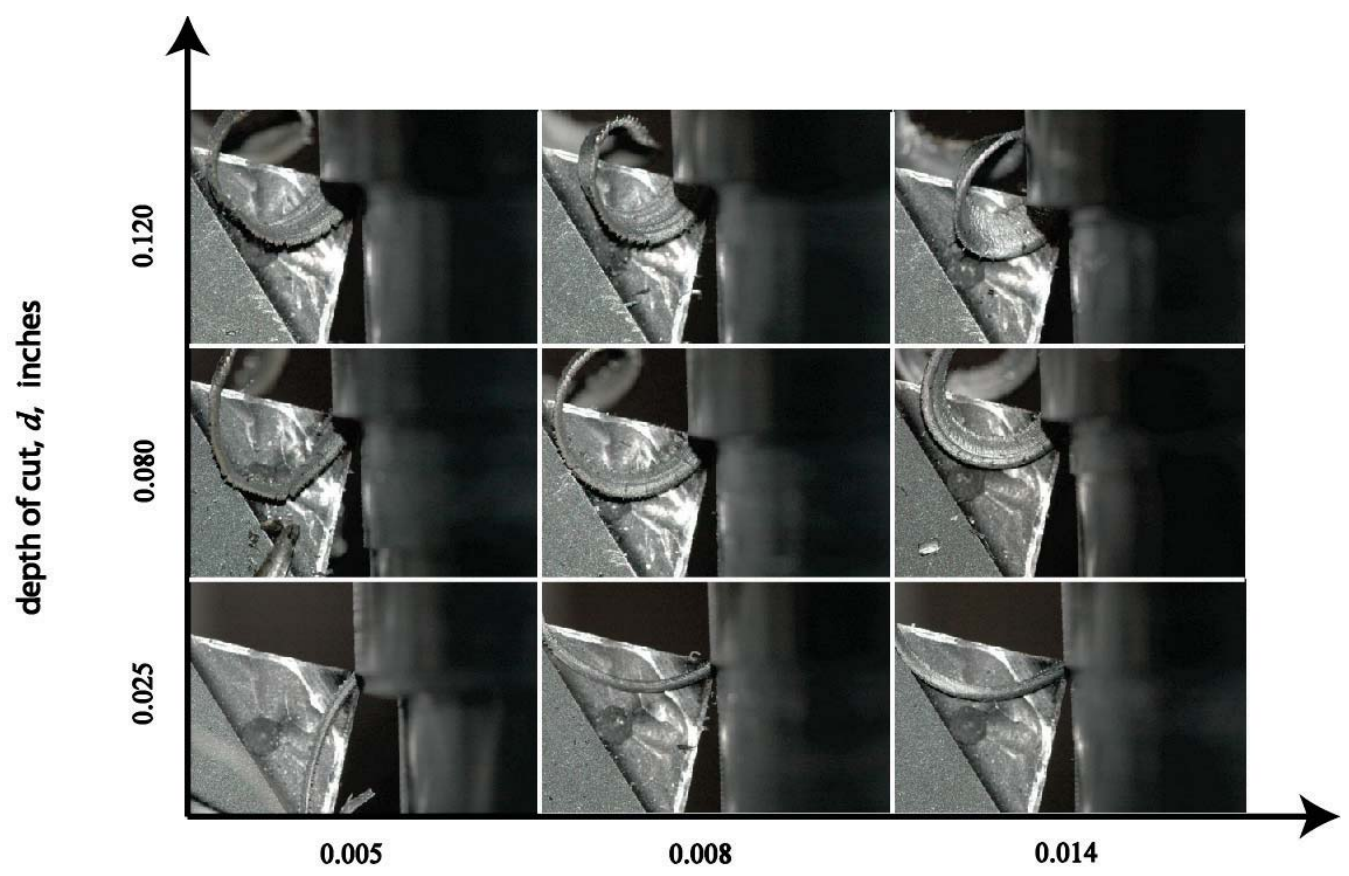

feed, $f$, ipr

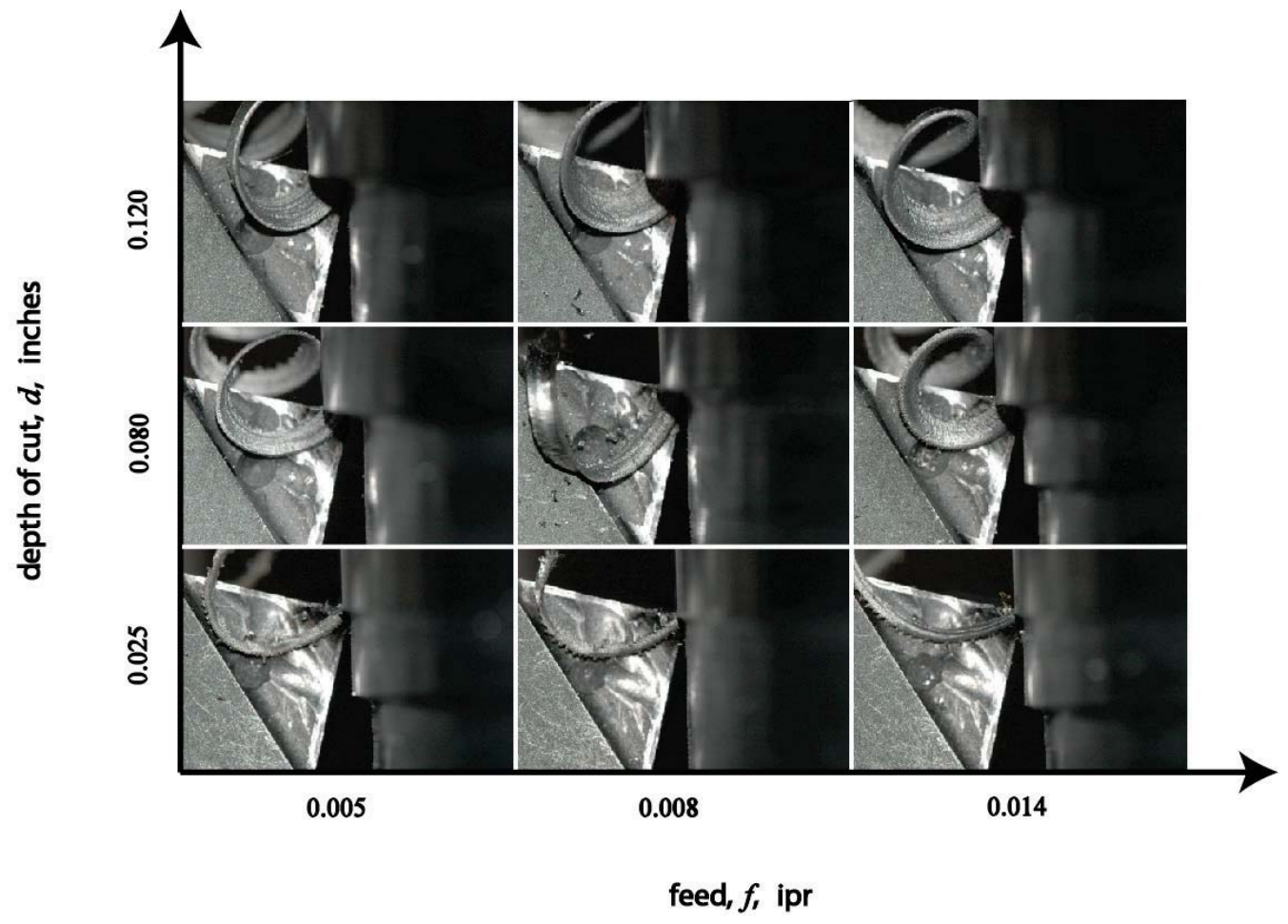

Figure E.6: Chip flow patterns for UCB tool (top) Replication \#2 (bottom) Replication \# 3; Work material: AISI 1018, $\psi_{r}=\mathbf{1 5}^{\circ}$. 


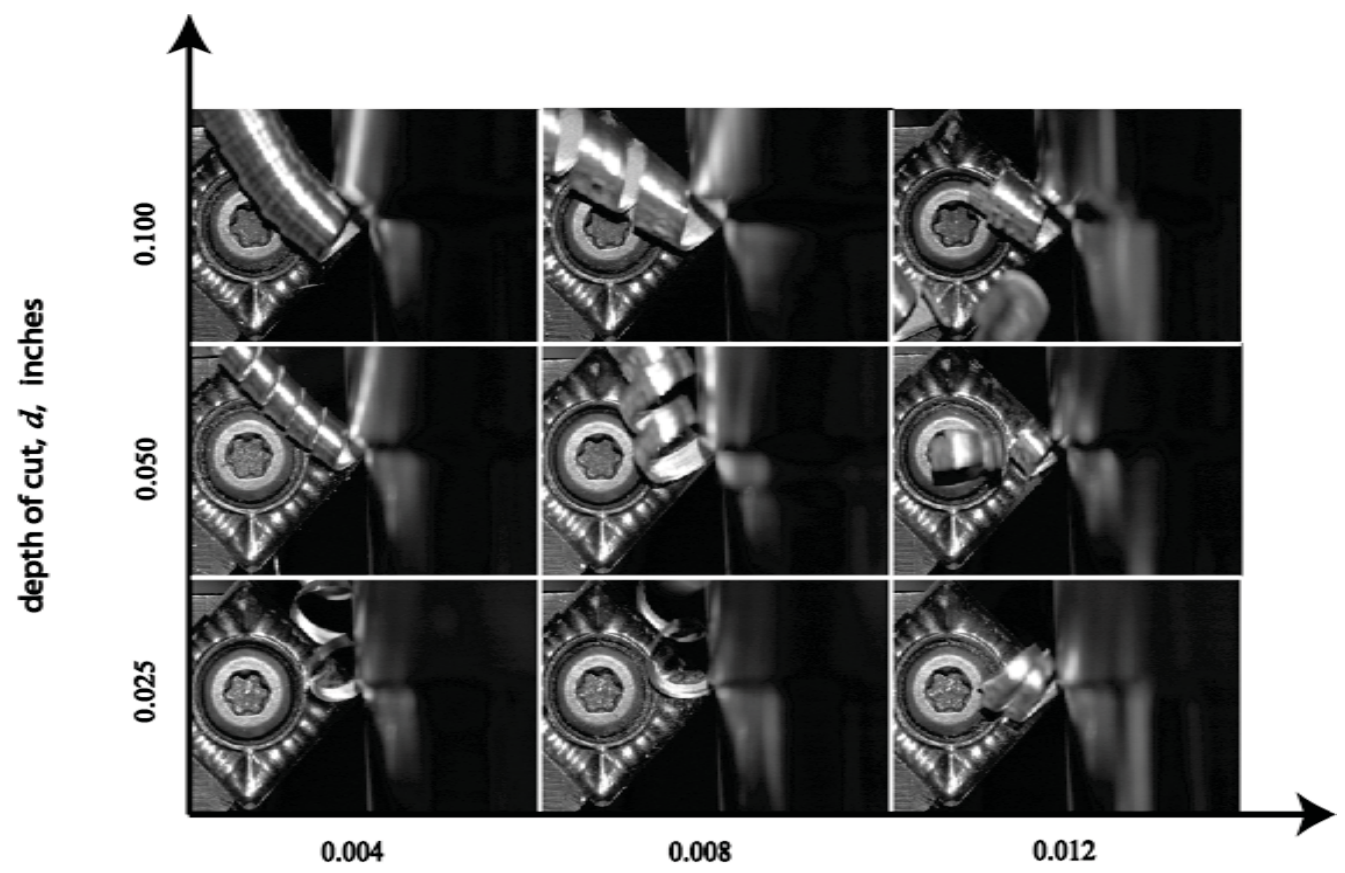

feed, $f$, ipr

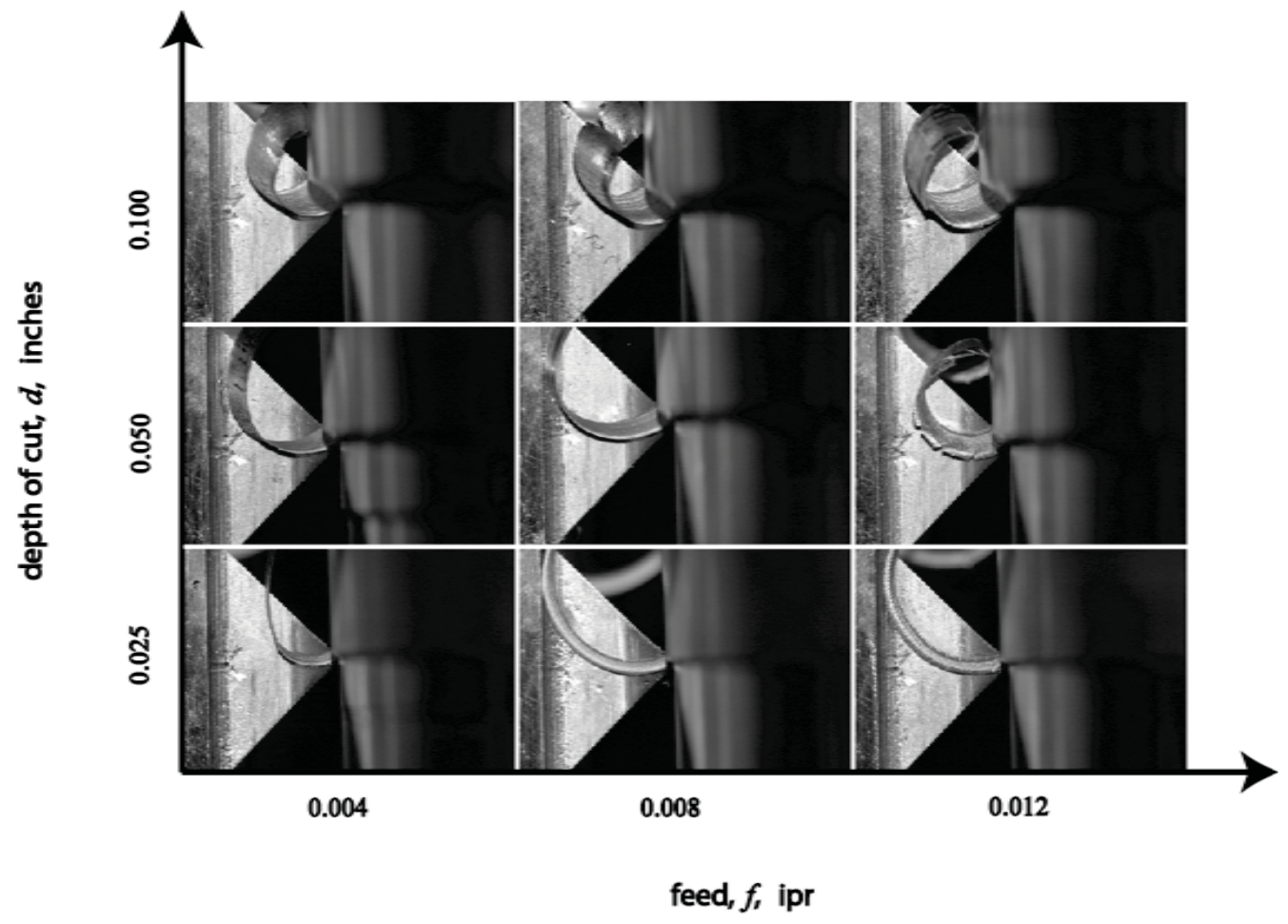

Figure E.7: Chip flow patterns for: (top) Replication \#1 - Korloy S-432 (bottom) Replication \#1 UCB tool; Work material: AL 2024, $\psi_{r}=\mathbf{4 5}^{\circ}$. 


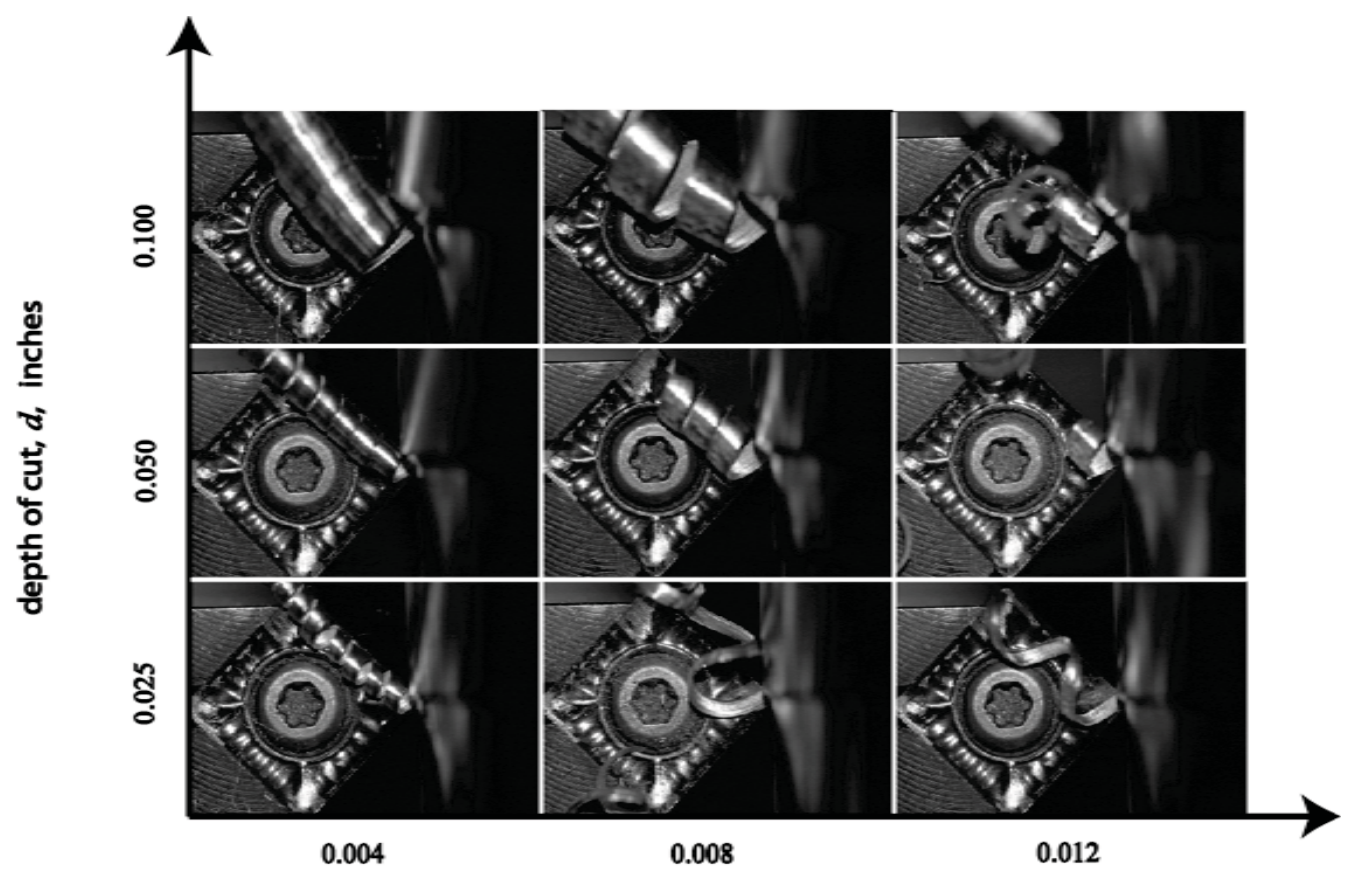

feed, $f$, ipr

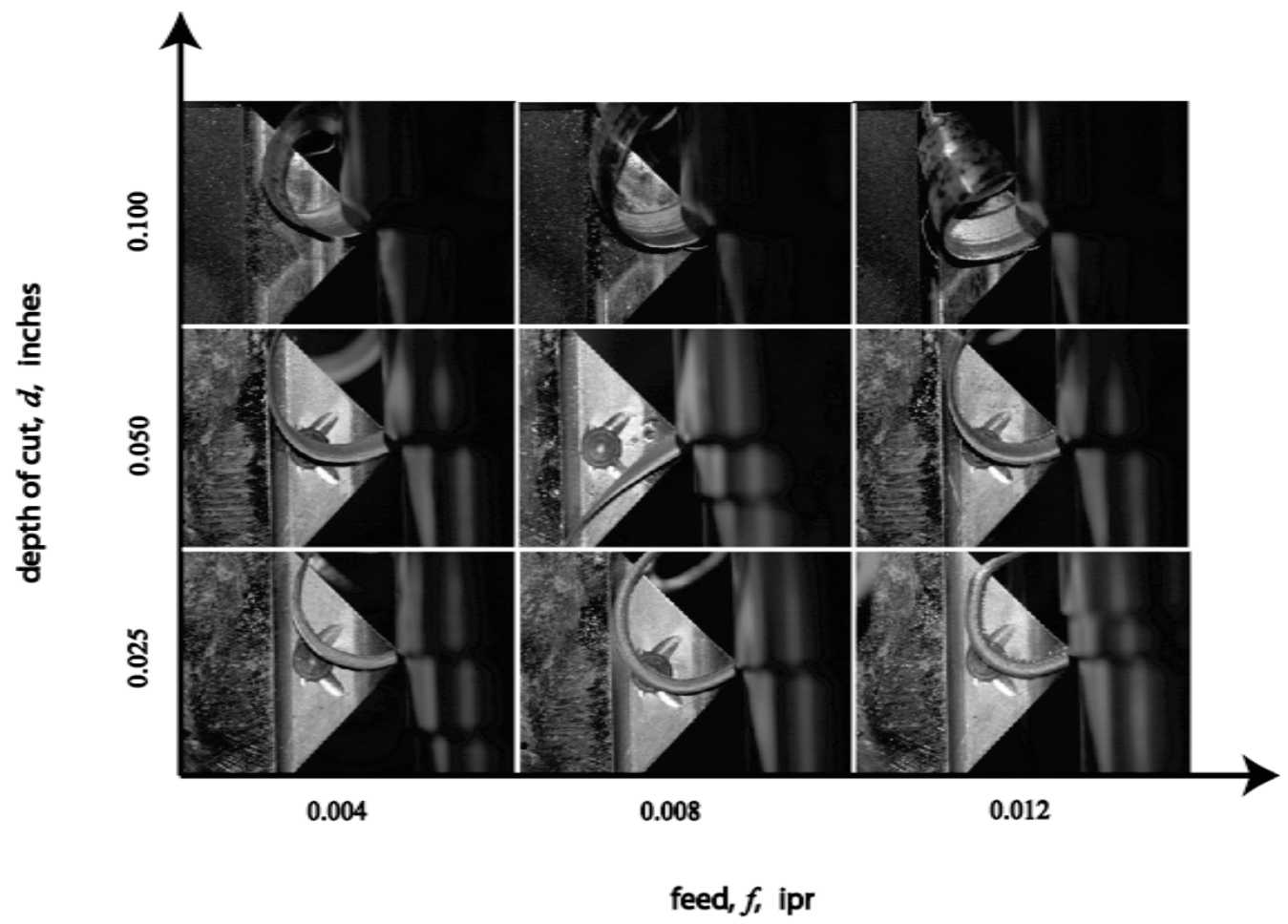

Figure E.8: Chip flow patterns for: (top) Replication \#2 - Korloy S-432 (bottom) Replication \#2 UCB tool; Work material: AL 2024, $\psi_{r}=4^{\circ}$. 


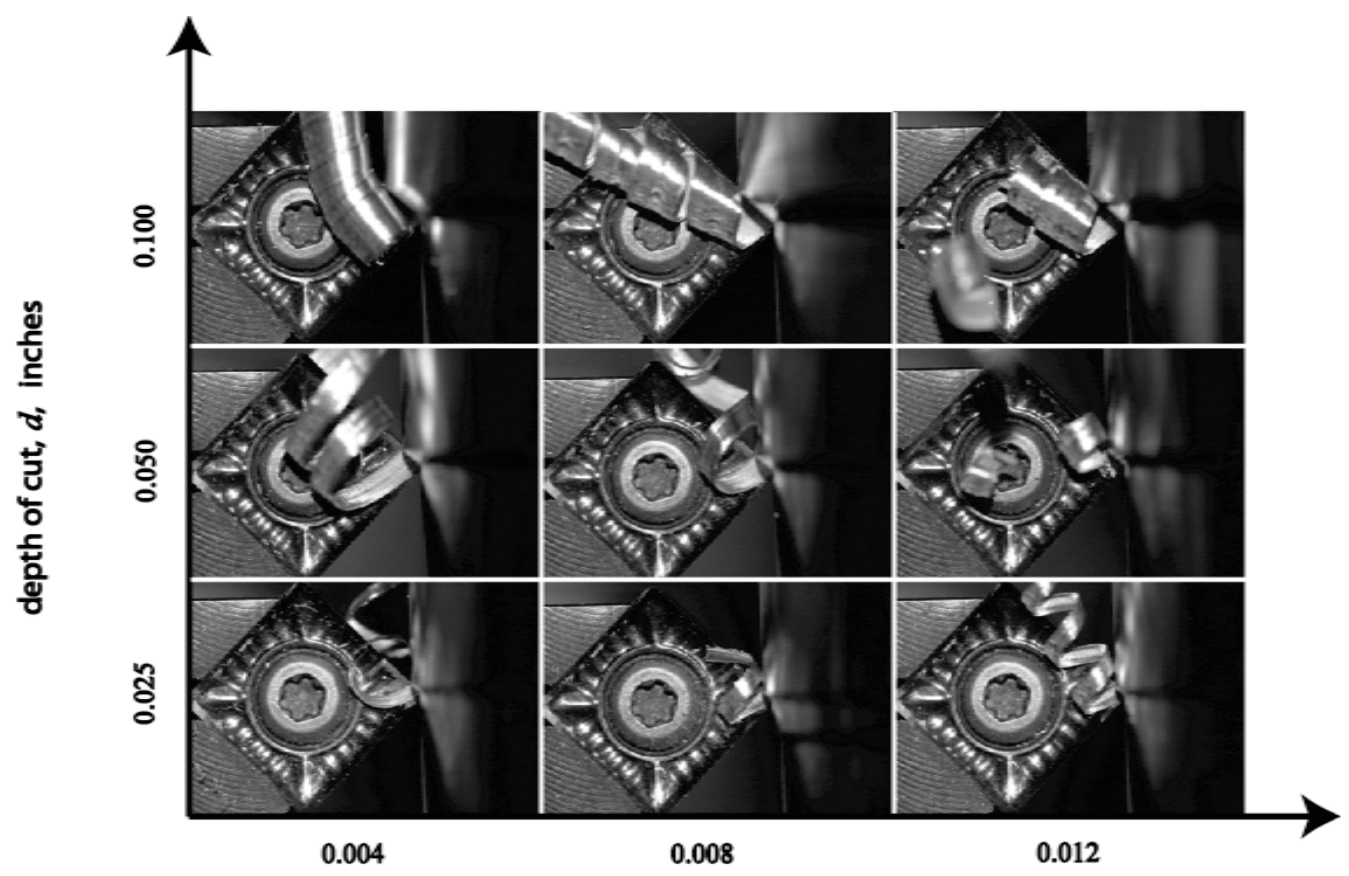

feed, $f$, ipr

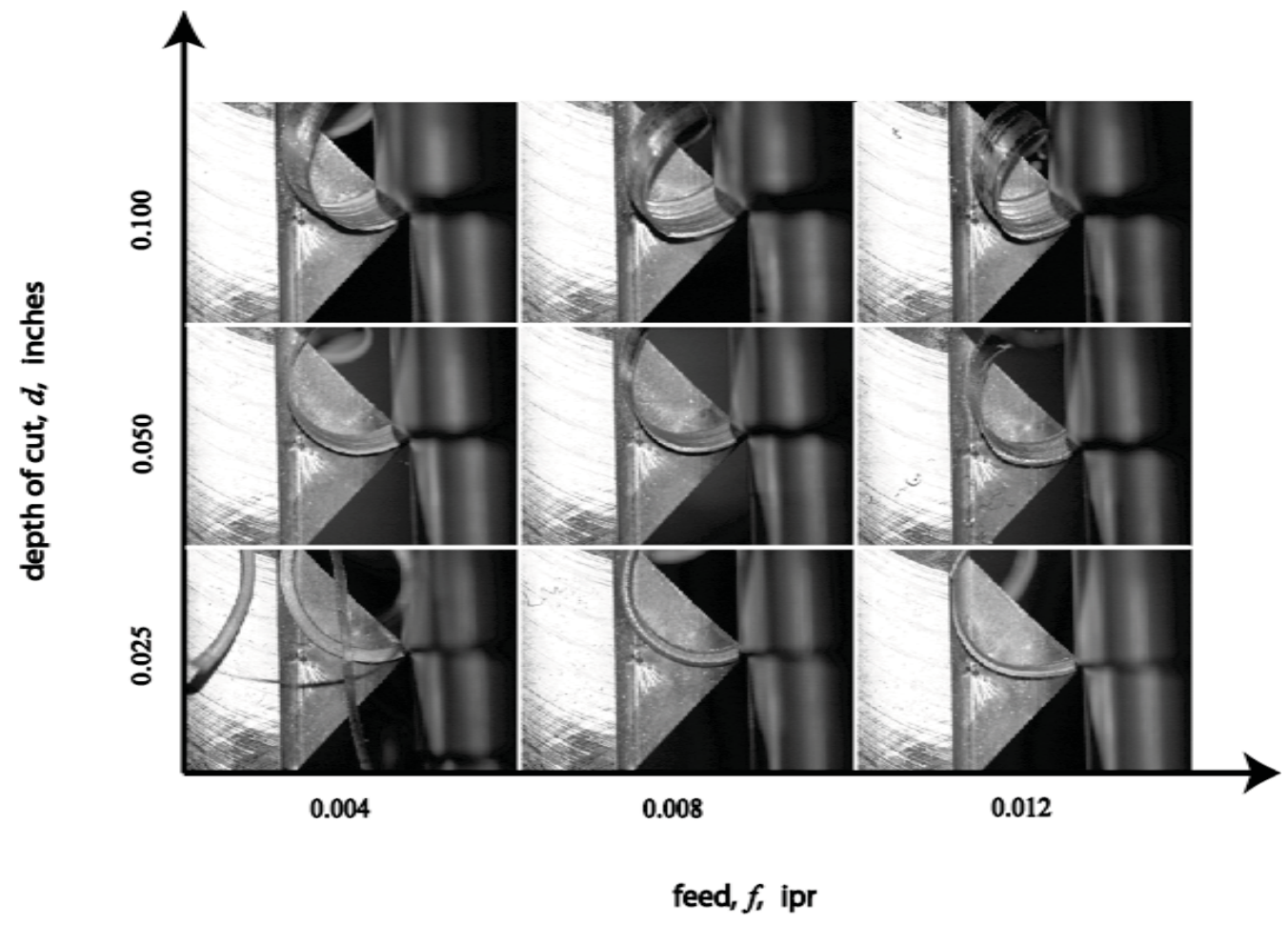

Figure E.9: Chip flow patterns for: (top) Replication \#3 - Korloy S-432 (bottom) Replication \#3 UCB tool; Work material: AL 2024, $\psi_{r}=\mathbf{4 5}^{\circ}$. 


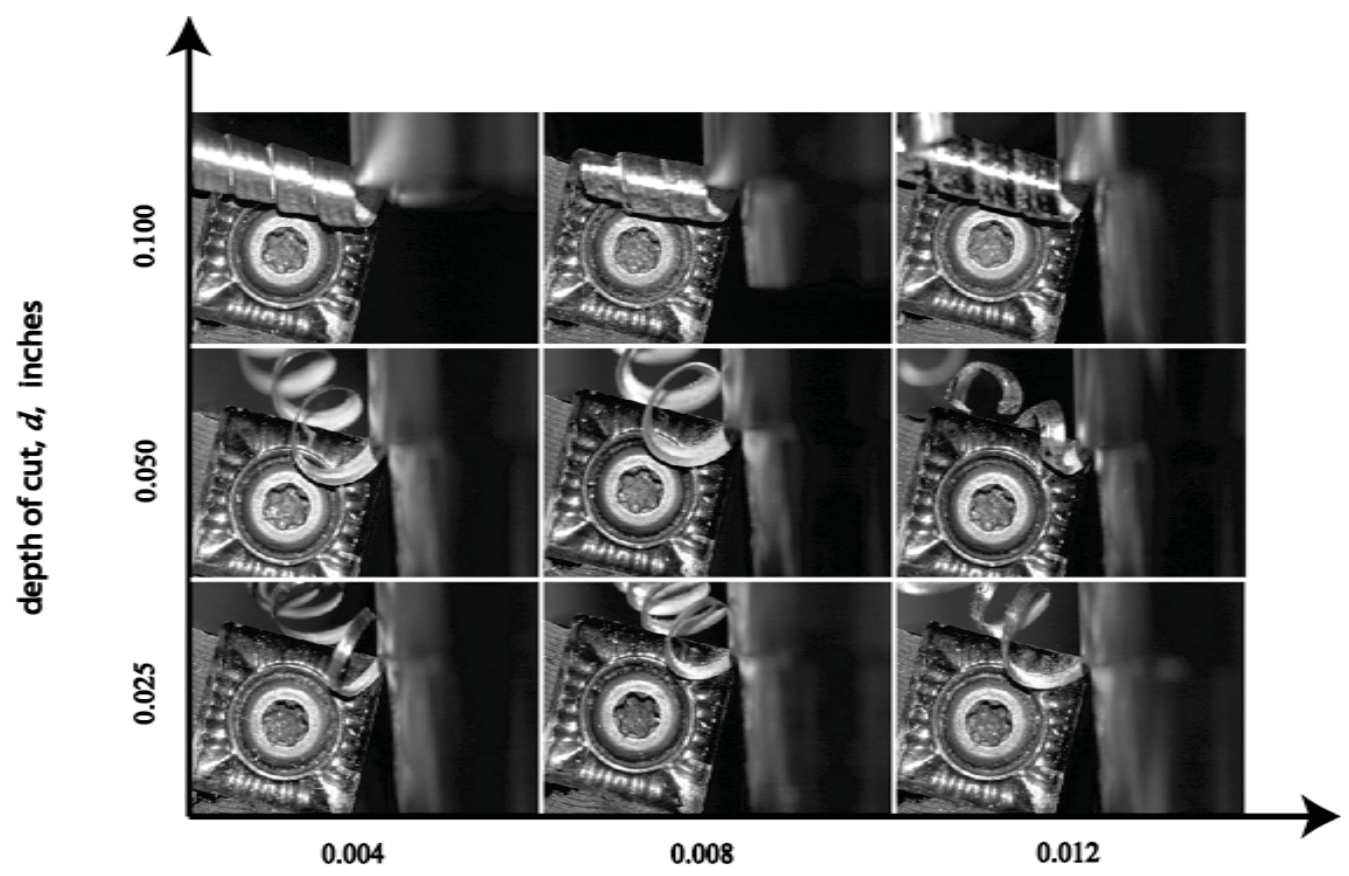

feed, $f$, ipr

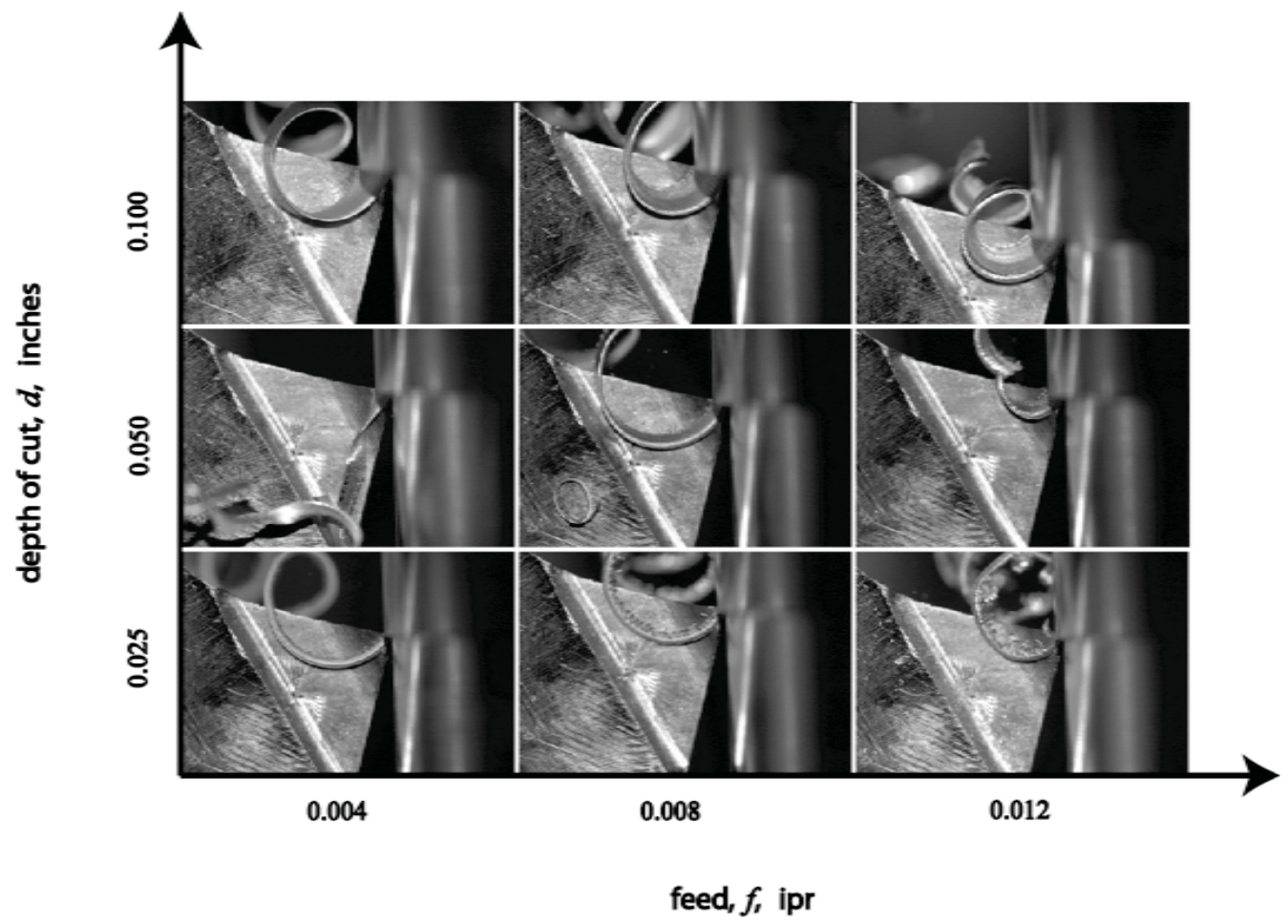

Figure E.10: Chip flow patterns for: (top) Replication \#1 - Korloy S-432 (bottom) Replication \#1 UCB tool; Work material: AL 2024, $\psi_{r}=15^{\circ}$. 


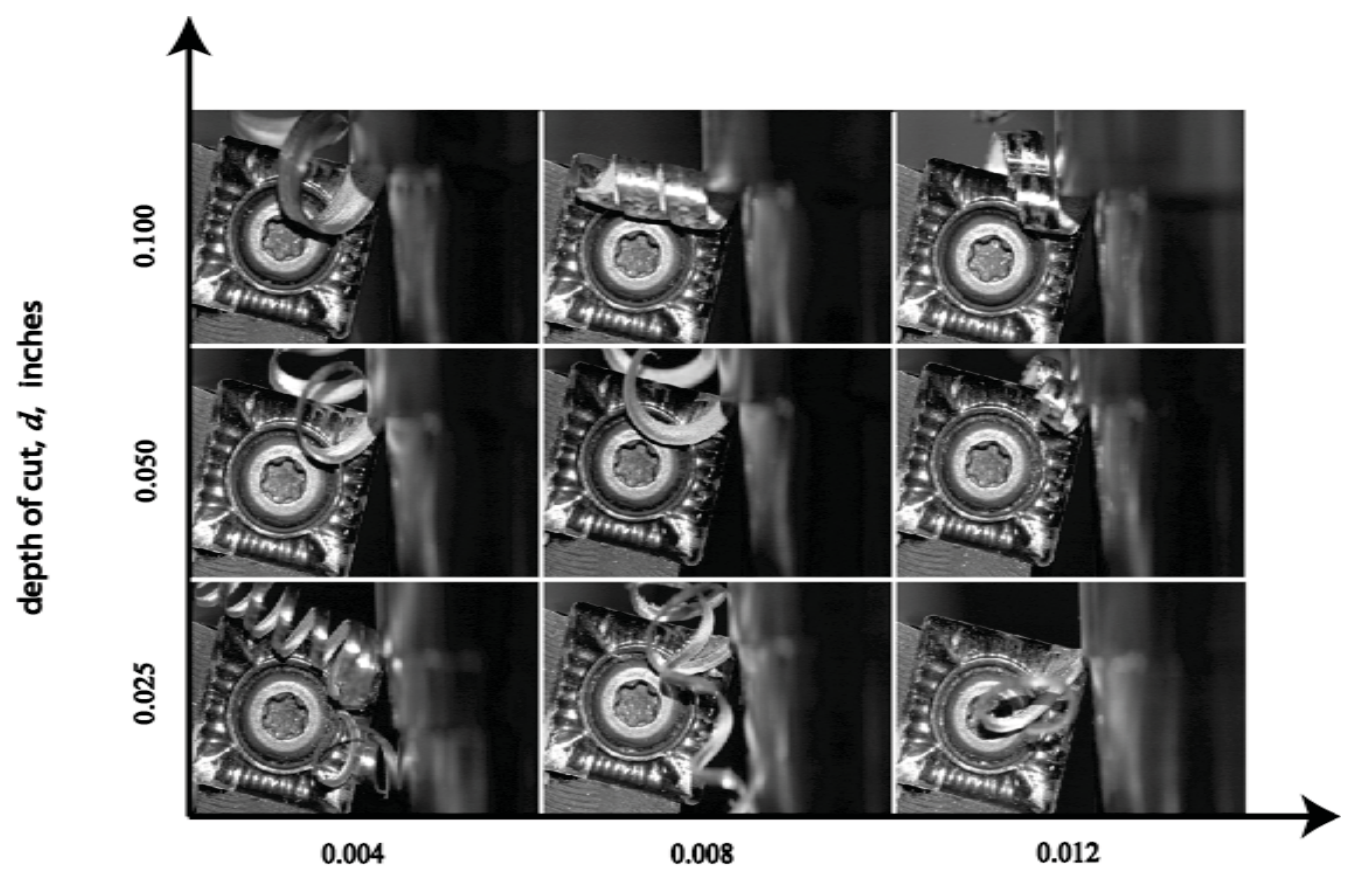

feed, $f$, ipr

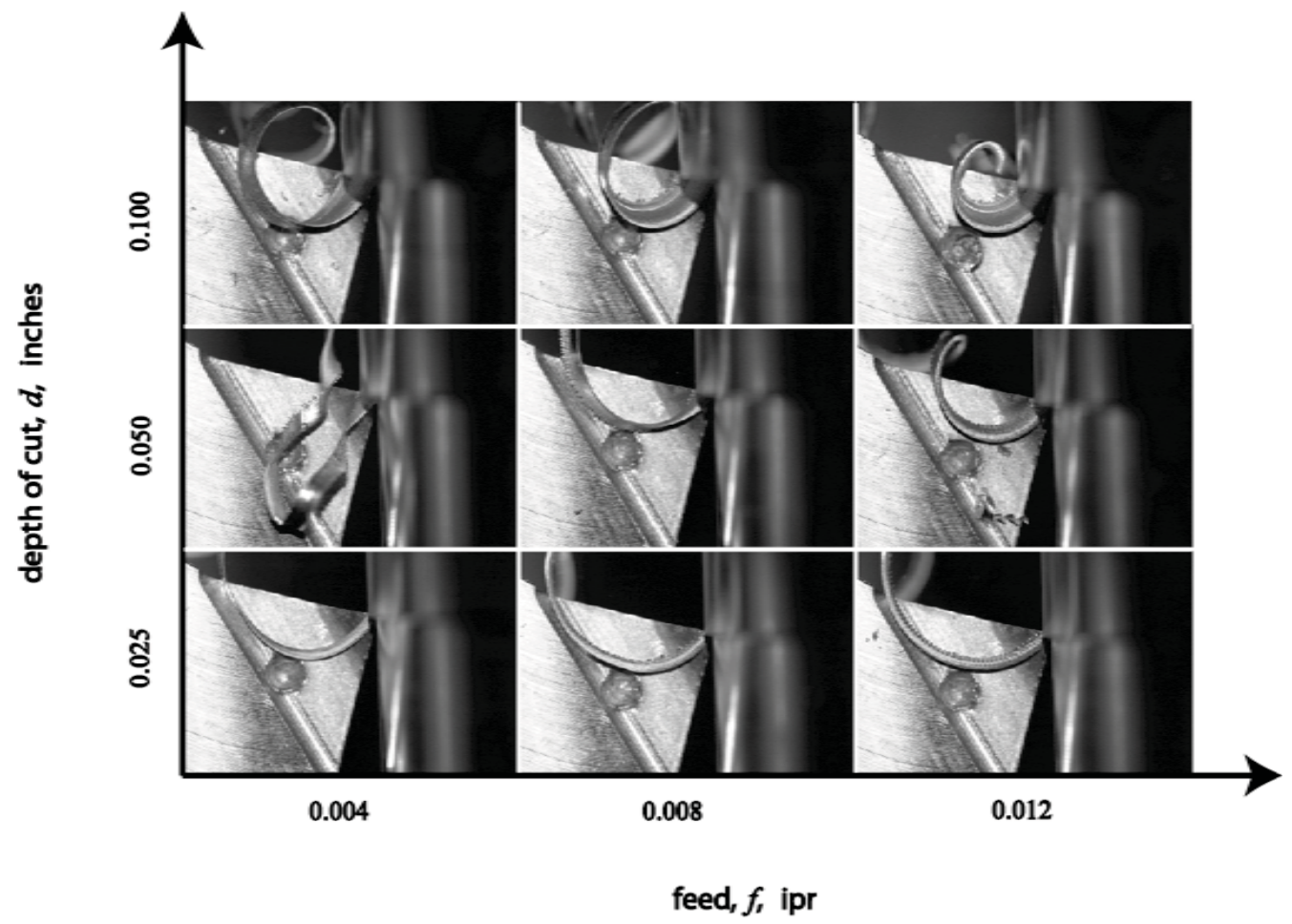

Figure E.11: Chip flow patterns for: (top) Replication \#2 - Korloy S-432 (bottom) Replication \#2 UCB tool; Work material: AL 2024, $\psi_{r}=15^{\circ}$. 


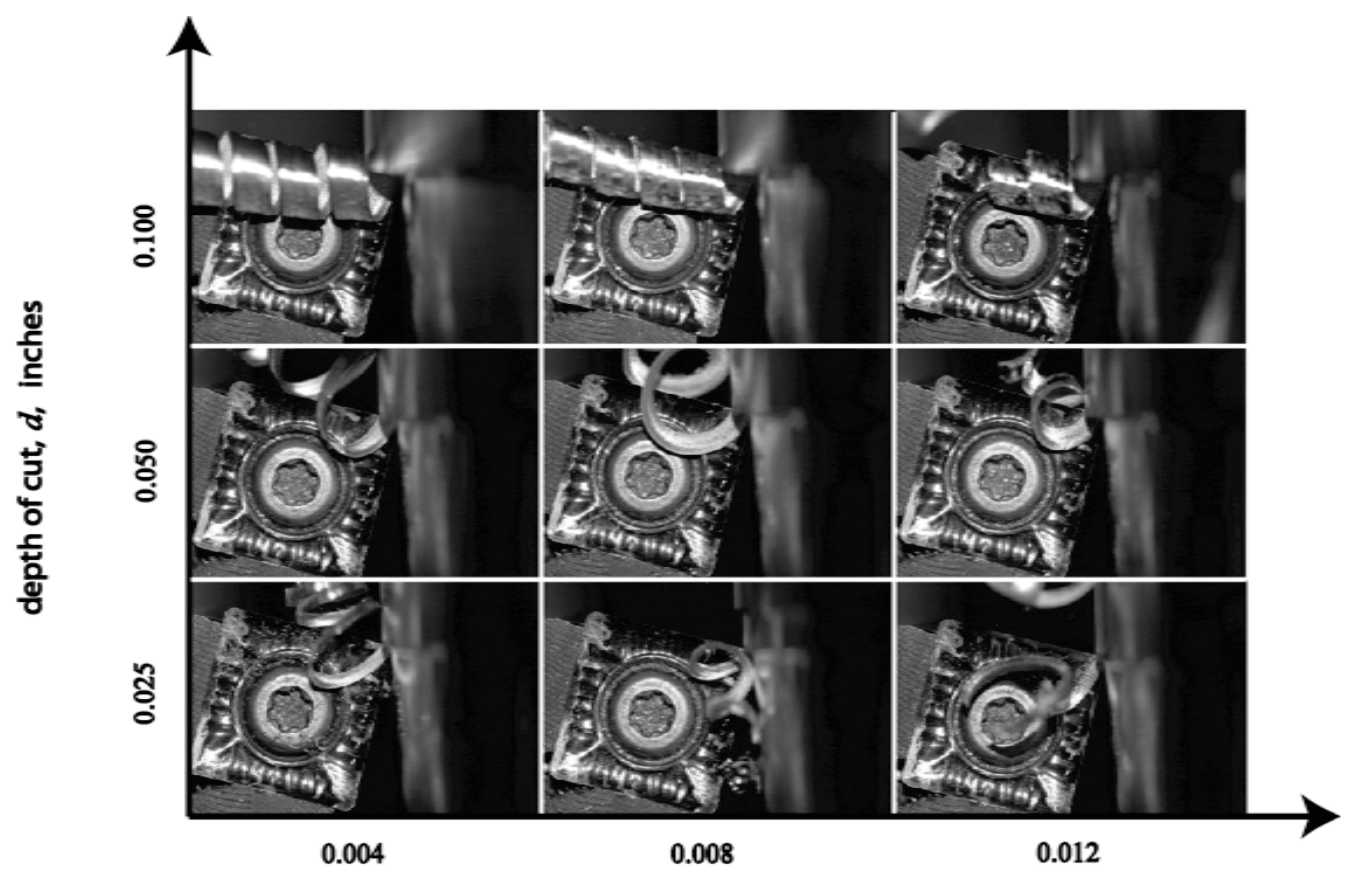

feed, $f$, ipr

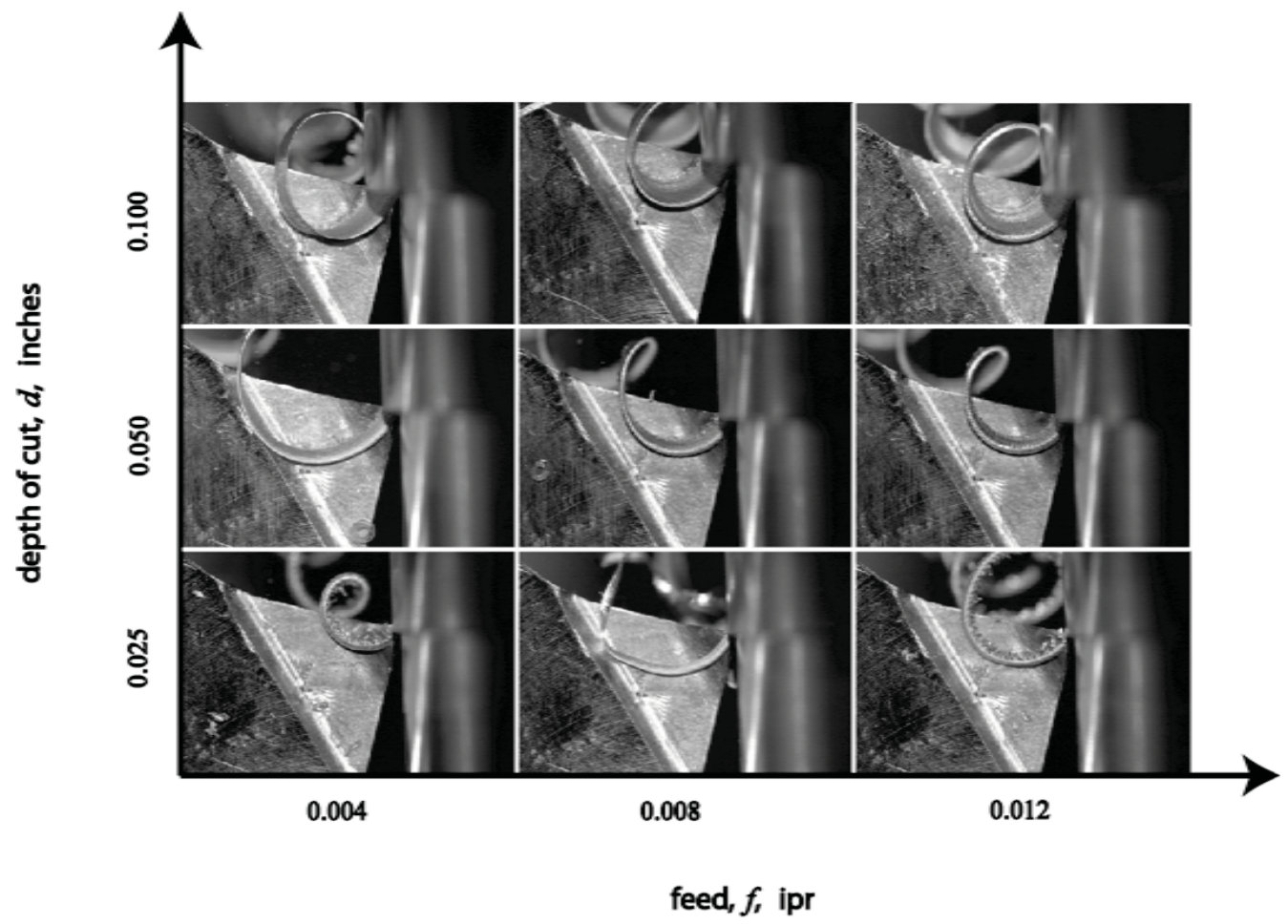

Figure E.12: Chip flow patterns for: (top) Replication \#3 - Korloy S-432 (bottom) Replication \#3 UCB tool; Work material: AL 2024, $\psi_{r}=\mathbf{1 5}^{0}$. 


\section{APPENDIX F}

\section{DESIGN OF TOP COVER FOR UCB TOOL}

Considerations for the design of the chip guiding channel in the UCB top cover was based on the actual chip cross sectional area produced by machining with the UCB tool. Chips produced by both AISI 1018 and AL2024 at $\psi_{r}=45^{\circ}$ and $\psi_{r}=15^{0}$ were measured for the thickness (measured at two edges of the chip) dimensions. Three chips for each of the depth of cut condition (all at the highest uncut chip thickness) were measured and the values tabulated as shown in Table F.1. The final width of the channel cross section was approximately $2 \mathrm{X}$ the chip width at the highest depth, $d=0.120$ inches $(\sim 0.250$ inches). The height of the channel was set at $3 X(\sim 0.120$ inches $)$ the thickest cut chip measurement of 0.0409 inches for the AISI 1018 steel at $\psi_{r}=15^{0}$. The channel top surface was provided the same shape as the tool rake face which then leads into a straight channel section. Adequate clearances were provided by machining reliefs at the front of the top cover and thereby preventing any interference of the top cover with the workpiece during machining. The channel ends in a spherical bowl, which is intended to bend the chip backwards into the hole provided on the UCB tool rake face. Figure F.1 shows details of the top cover design. 
Table F.1: Measurements of chip thickness for UCB tool.

\begin{tabular}{|c|c|c|c|c|c|c|c|}
\hline \multicolumn{8}{|c|}{$\begin{array}{l}\text { Material: AISI } 1018 \\
\psi=45 \mathrm{deg} \\
\mathrm{f}=0.014 \mathrm{ipr}\end{array}$} \\
\hline \multirow{3}{*}{$\begin{array}{l}\text { Depth of cut } \\
\quad \text { (inch) }\end{array}$} & \multicolumn{7}{|c|}{ Measured Chip Thickness (inch) } \\
\hline & \multicolumn{2}{|c|}{ Chip 1} & \multicolumn{2}{|c|}{ Chip 2} & \multicolumn{2}{|c|}{ Chip 3} & \multirow{2}{*}{$\begin{array}{l}\text { Average Chip } \\
\text { Thickness (inch) }\end{array}$} \\
\hline & Side 1 & Side 2 & Side 1 & Side 2 & Side 1 & Side 2 & \\
\hline 0.025 & 0.0269 & 0.0281 & 0.0276 & 0.0288 & 0.0259 & 0.0271 & 0.0274 \\
\hline 0.08 & 0.0278 & 0.035 & 0.031 & 0.034 & 0.029 & 0.035 & 0.0320 \\
\hline 0.12 & 0.038 & 0.04 & 0.036 & 0.041 & 0.034 & 0.037 & 0.0377 \\
\hline \multicolumn{8}{|c|}{$\begin{array}{l}\text { Material: AISI } 1 \\
\psi=15 \mathrm{deg} \\
\mathrm{f}=0.014 \mathrm{ipr}\end{array}$} \\
\hline \multirow{3}{*}{$\begin{array}{l}\text { Depth of cut } \\
\quad \text { (inch) }\end{array}$} & \multicolumn{7}{|c|}{ Measured Chip Thickness (inch) } \\
\hline & \multicolumn{2}{|c|}{ Chip 1} & \multicolumn{2}{|c|}{ Chip 2} & \multicolumn{2}{|c|}{ Chip 3} & \multirow{2}{*}{$\begin{array}{l}\text { Average Chip } \\
\text { Thickness (inch) }\end{array}$} \\
\hline & Side 1 & Side 2 & Side 1 & Side 2 & Side 1 & Side 2 & \\
\hline 0.025 & 0.0274 & 0.0299 & 0.027 & 0.0282 & 0.0241 & 0.0269 & 0.0273 \\
\hline 0.08 & 0.0308 & 0.0312 & 0.0292 & 0.0324 & 0.0261 & 0.0298 & 0.0299 \\
\hline 0.12 & 0.0415 & 0.0423 & 0.0356 & 0.04313 & 0.0386 & 0.0441 & 0.0409 \\
\hline \multicolumn{8}{|c|}{$\begin{array}{l}\text { Material: AL } 20 \\
\psi=45 \mathrm{deg} \\
\mathrm{f}=0.012 \mathrm{ipr}\end{array}$} \\
\hline \multirow{3}{*}{$\begin{array}{l}\text { Depth of cut } \\
\quad \text { (inch) }\end{array}$} & \multicolumn{7}{|c|}{ Measured Chip Thickness (inch) } \\
\hline & \multicolumn{2}{|c|}{ Chip 1} & \multicolumn{2}{|c|}{ Chip 2} & \multicolumn{2}{|c|}{ Chip 3} & \multirow{2}{*}{$\begin{array}{l}\text { Average Chip } \\
\text { Thickness (inch) }\end{array}$} \\
\hline & Side 1 & Side 2 & Side 1 & Side 2 & Side 1 & Side 2 & \\
\hline 0.025 & 0.0157 & 0.0163 & 0.0142 & 0.0151 & 0.0141 & 0.0158 & 0.0152 \\
\hline 0.05 & 0.0159 & 0.0161 & 0.015 & 0.0156 & 0.0153 & 0.0171 & 0.0158 \\
\hline 0.1 & 0.0191 & 0.0241 & 0.0168 & 0.0183 & 0.0186 & 0.0194 & 0.0194 \\
\hline \multicolumn{8}{|c|}{$\begin{array}{l}\text { Material: AL } 20 \\
\psi=15 \mathrm{deg} \\
\mathrm{f}=0.012 \mathrm{ipr}\end{array}$} \\
\hline \multirow{3}{*}{$\begin{array}{l}\text { Depth of cut } \\
\quad \text { (inch) }\end{array}$} & \multicolumn{7}{|c|}{ Measured Chip Thickness (inch) } \\
\hline & \multicolumn{2}{|c|}{ Chip 1} & \multicolumn{2}{|c|}{ Chip 2} & \multicolumn{2}{|c|}{ Chip 3} & \multirow{2}{*}{$\begin{array}{l}\text { Average Chip } \\
\text { Thickness (inch) }\end{array}$} \\
\hline & Side 1 & Side 2 & Side 1 & Side 2 & Side 1 & Side 2 & \\
\hline 0.025 & 0.0178 & 0.0181 & 0.0166 & 0.0161 & 0.0157 & 0.0171 & 0.0169 \\
\hline 0.05 & 0.0187 & 0.0194 & 0.0178 & 0.0191 & 0.0196 & 0.0206 & 0.0192 \\
\hline 0.1 & 0.0208 & 0.021 & 0.0217 & 0.0223 & 0.0198 & 0.0209 & 0.0211 \\
\hline
\end{tabular}



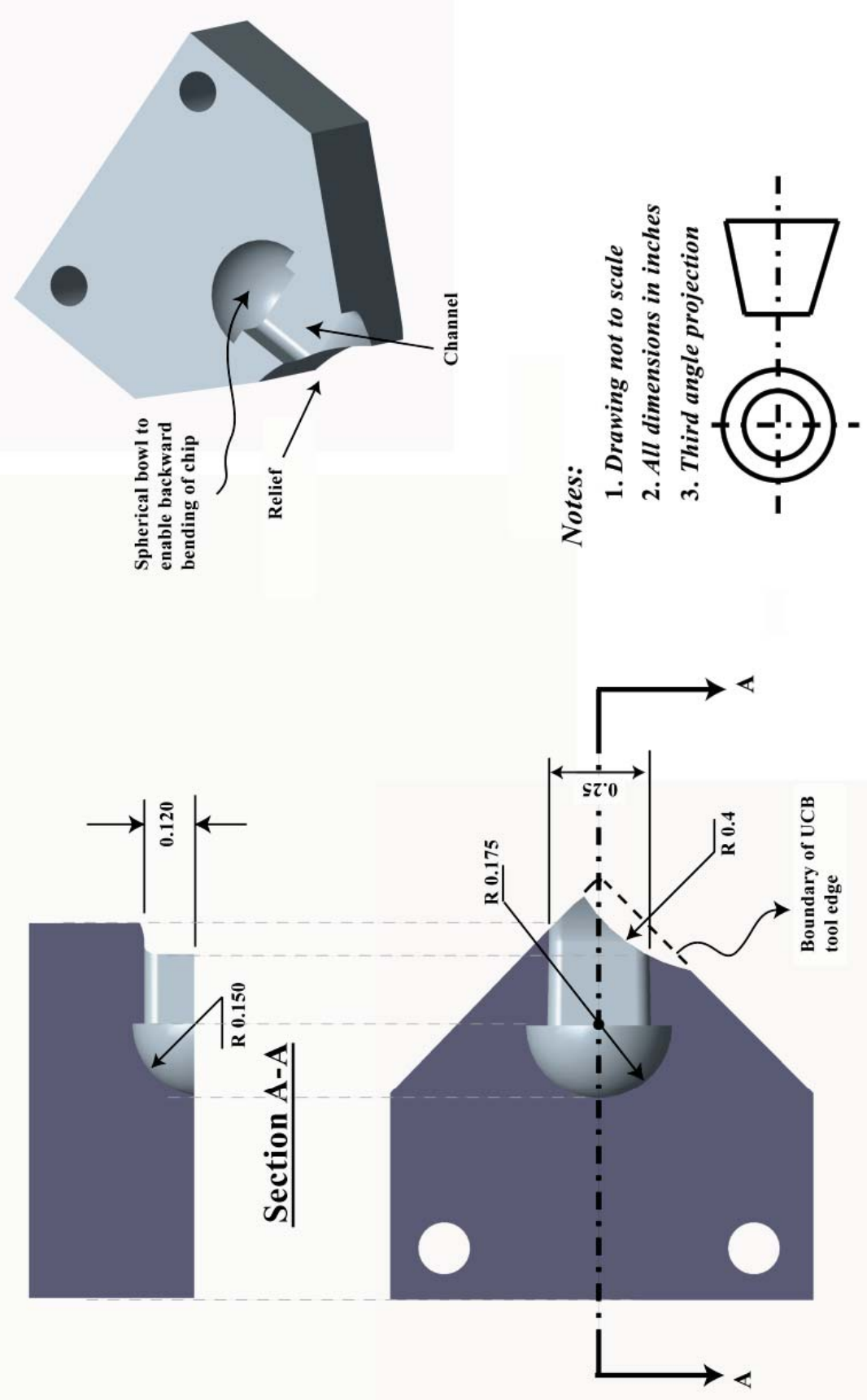

Figure F.1: Detailed drawing of the UCB top cover. 


\section{APPENDIX G}

\section{REPLICATED RESULTS OF CHIP BREAKING TESTS}

(this page intentionally left blank) 

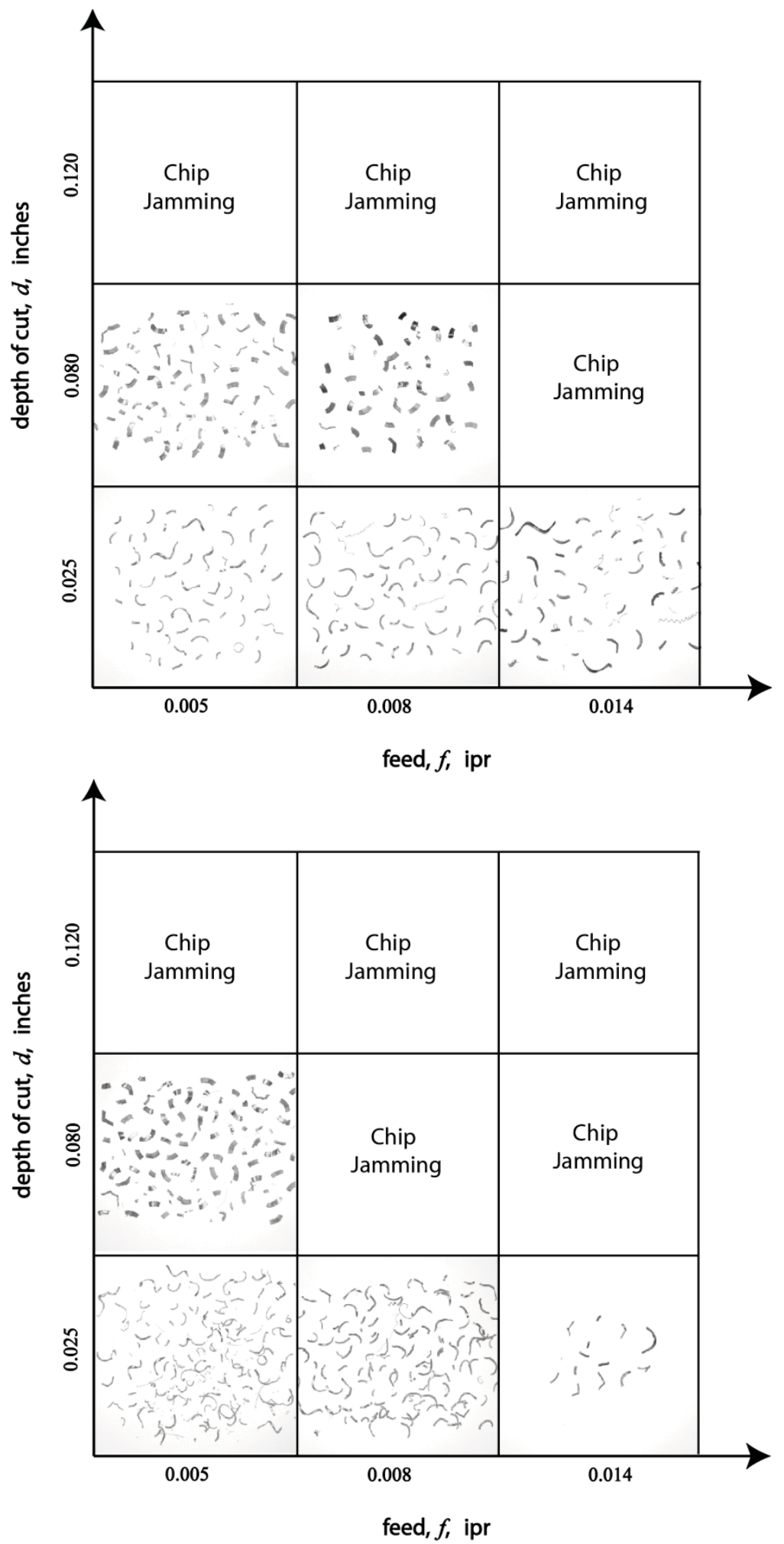


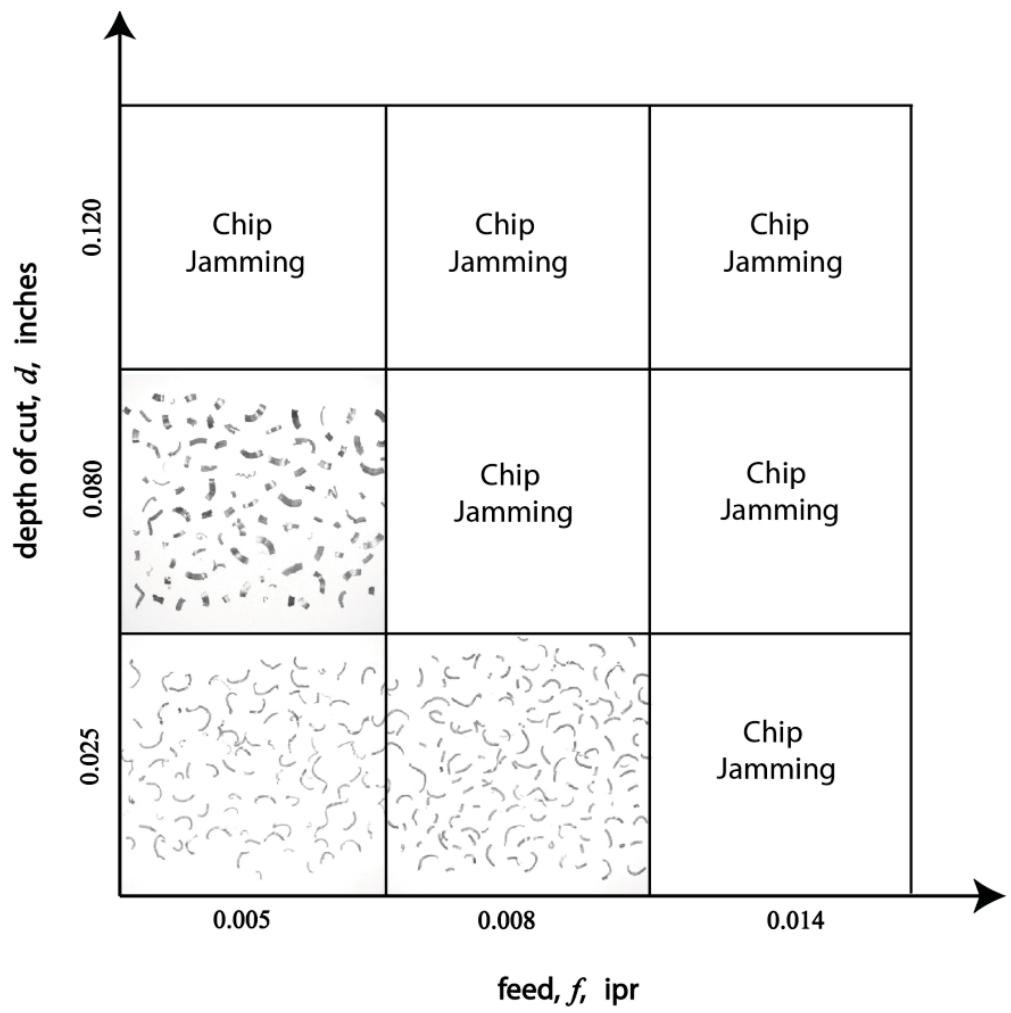

Figure G.1: Chip charts for Flat tool with the top guidance system (Previous page top) Replication \#1 ( Previous page bottom) Replication \# 2 (This page) Replication \#3;

Work material: AISI 1018. 

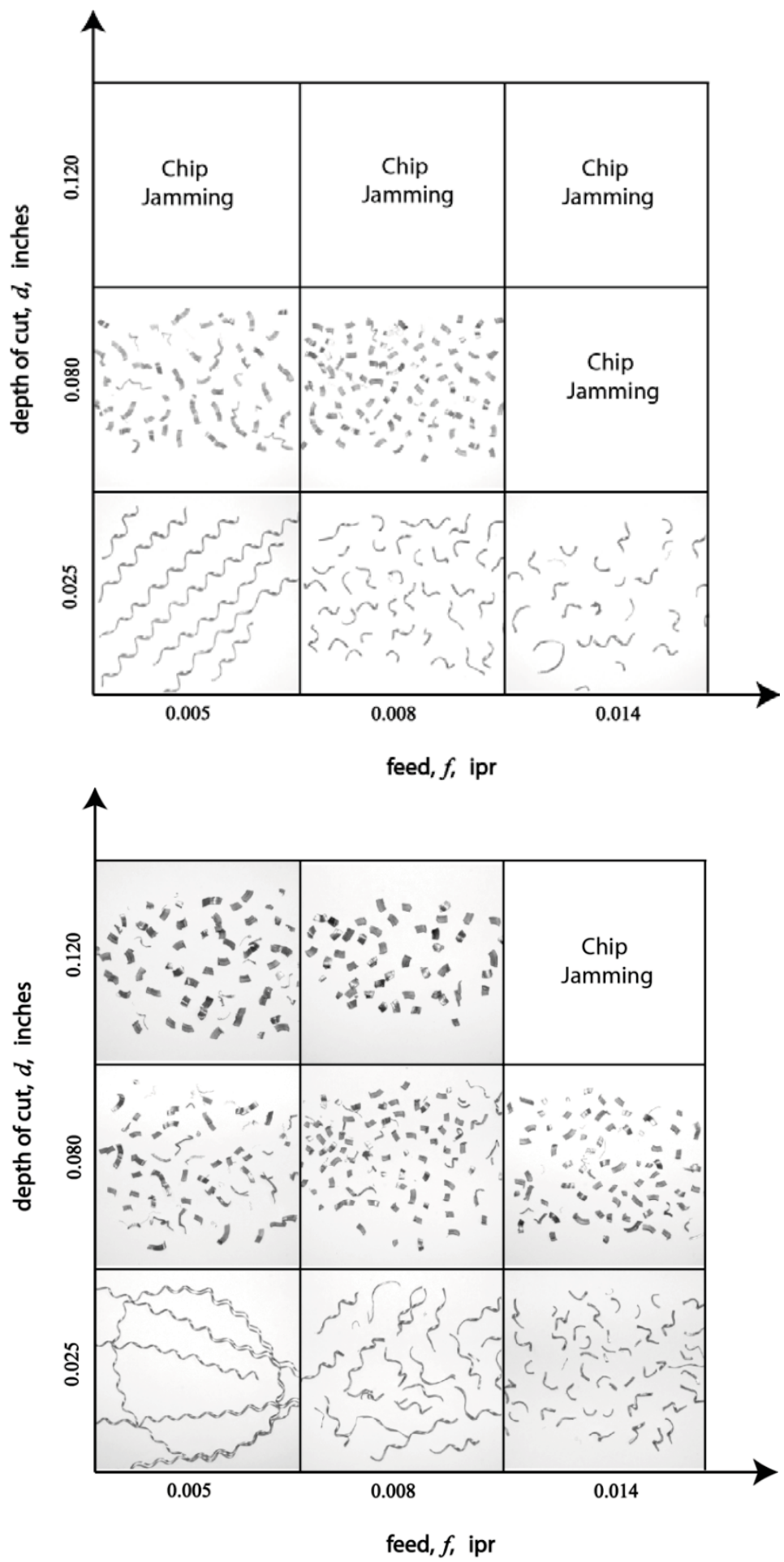

Figure G.2: Chip charts with the top guidance system for: (top) Replication \#2 - UCB (R) (bottom) Replication \#2 - UCB tool; Work material: AISI 1018. 

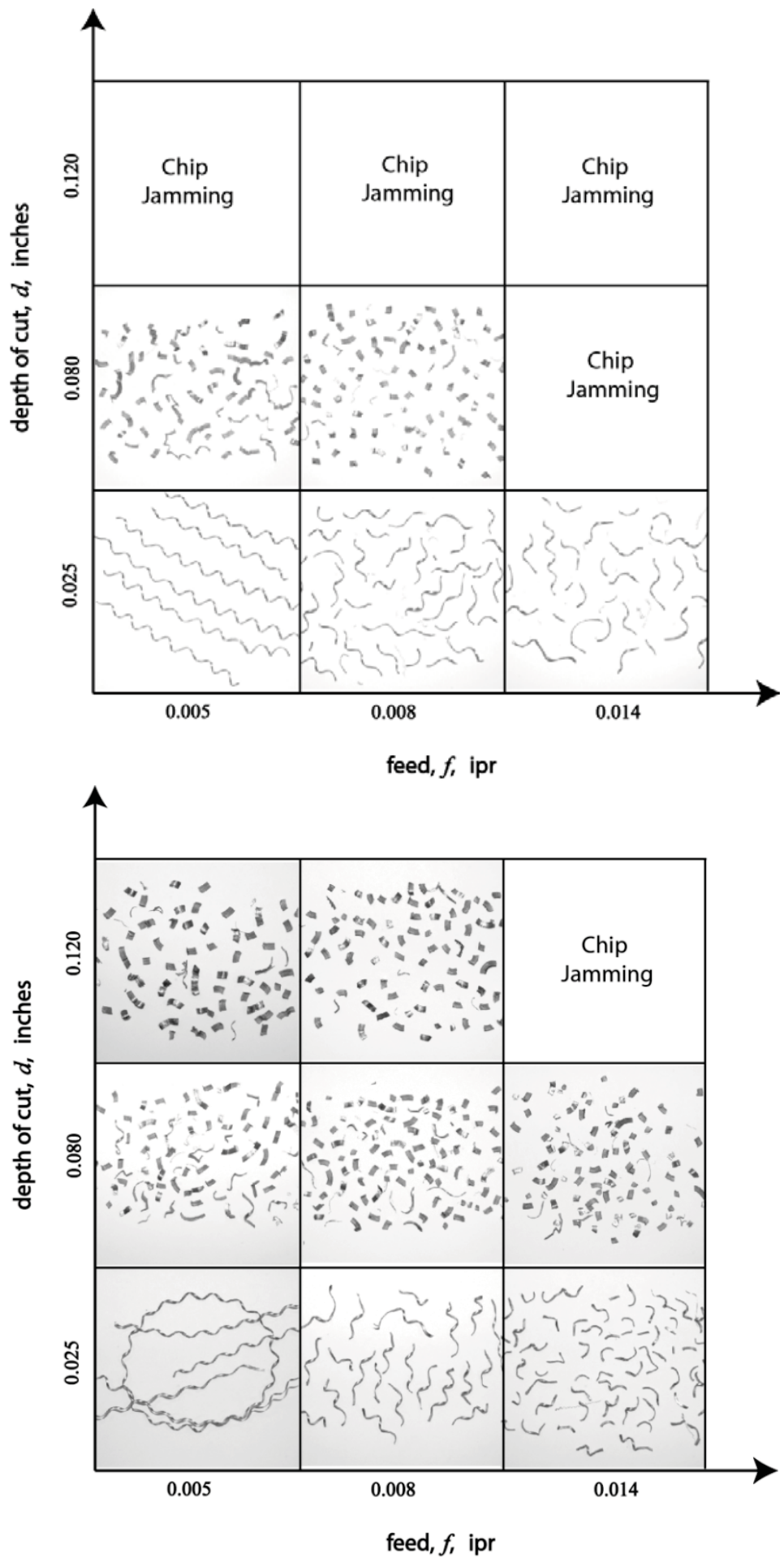

Figure G.3: Chip charts with the top guidance system for: (top) Replication \#3 - UCB (R) (bottom) Replication \#3 - UCB tool; Work material: AISI 1018. 

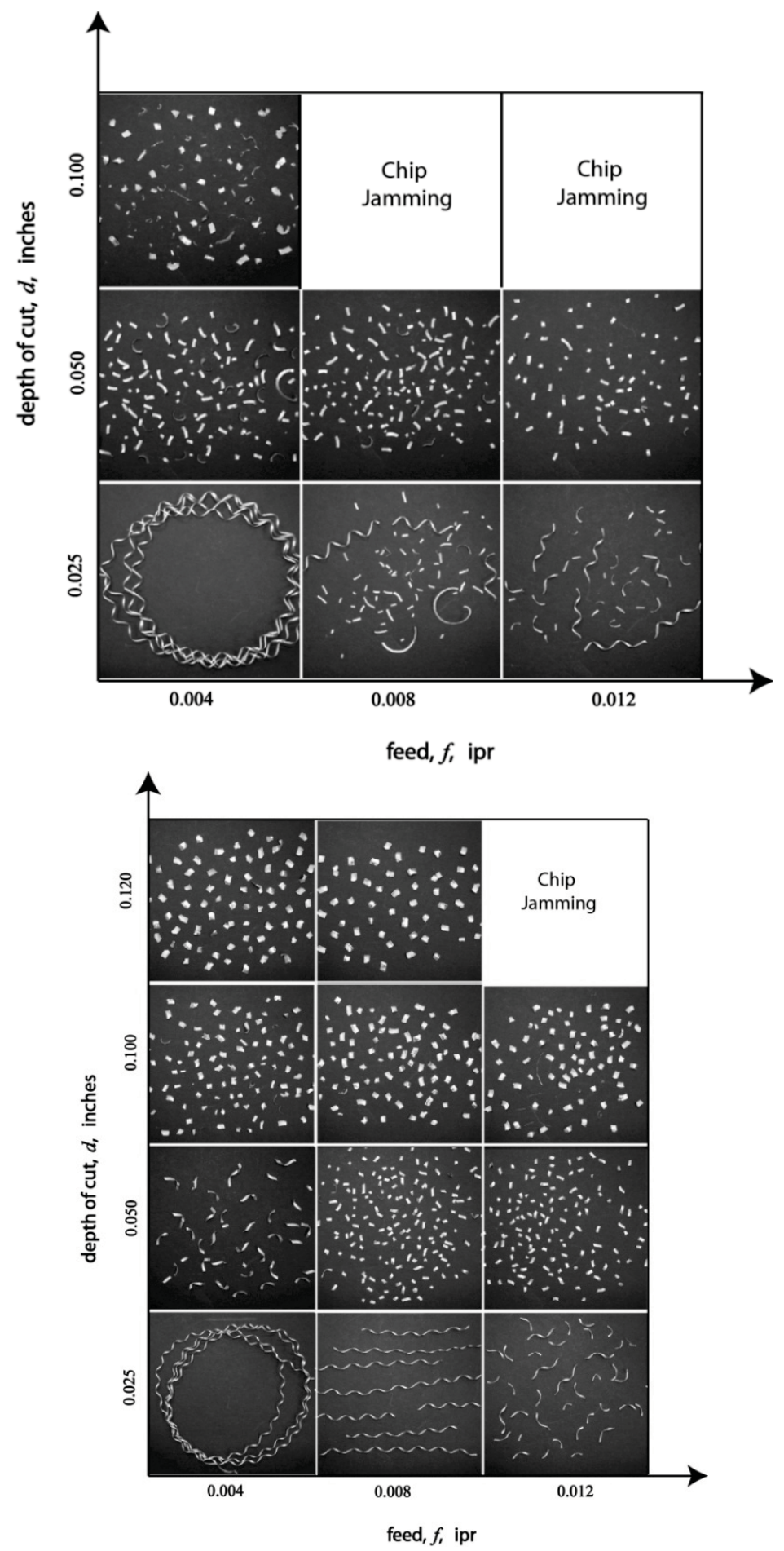

Figure G.4: Chip charts with the top guidance system for: (top) Replication \#2 - UCB (R) (bottom) Replication \#2 - UCB tool; Work material: AL 2024. 

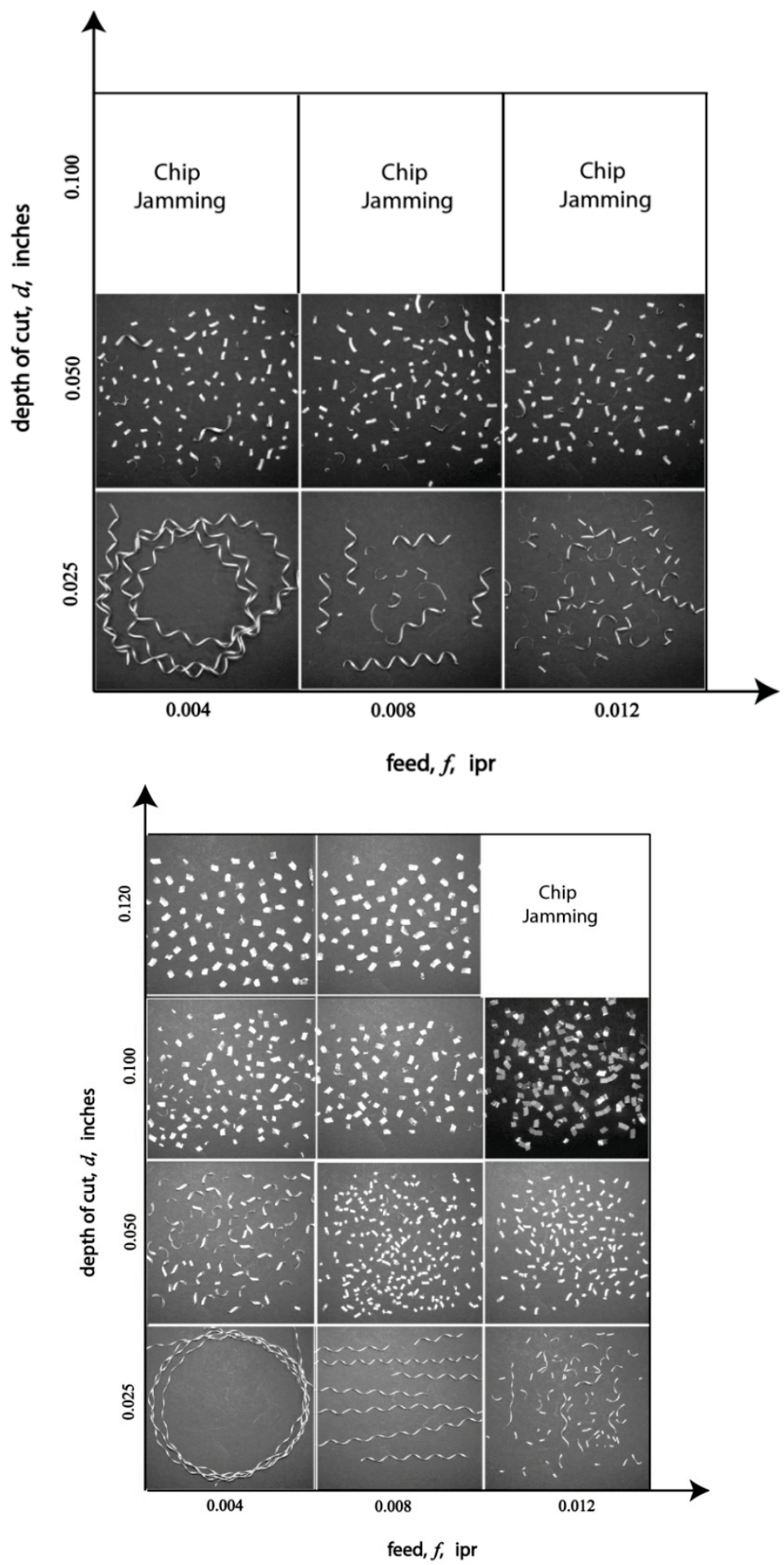

Figure G.5: Chip charts with the top guidance system for: (top) Replication \#3 - UCB (R) (bottom) Replication \#3 - UCB tool; Work material: AL 2024. 


\section{APPENDIX H}

\section{REPLICATED RESULTS OF CHIP BREAKING TESTS \\ WITH COOLANT APPLICATION}

(this page intentionally left blank) 

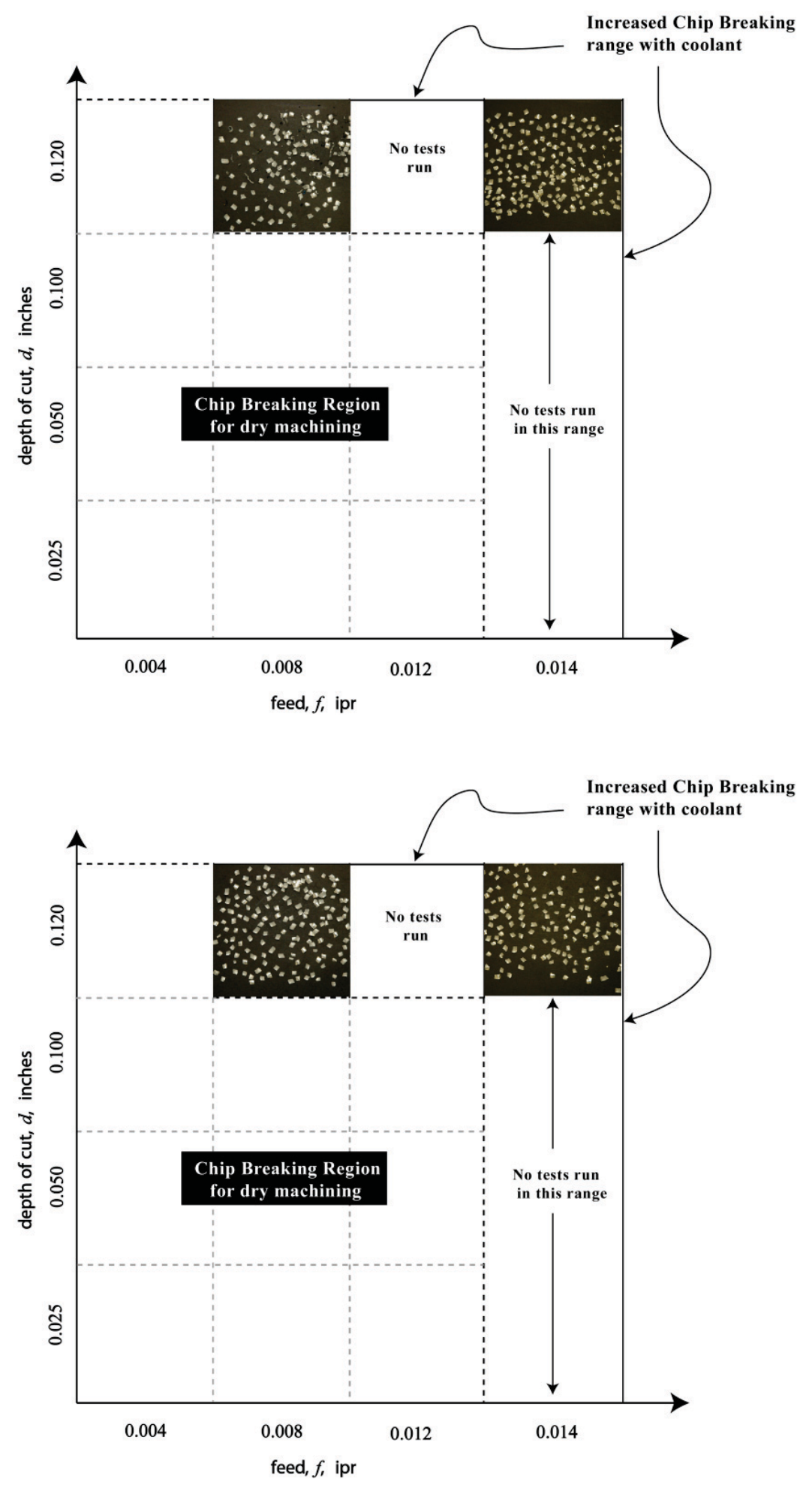

Figure H.1: UCB chip breaking tests with coolant (top) Replication \#2 (bottom) Replication \#3. 

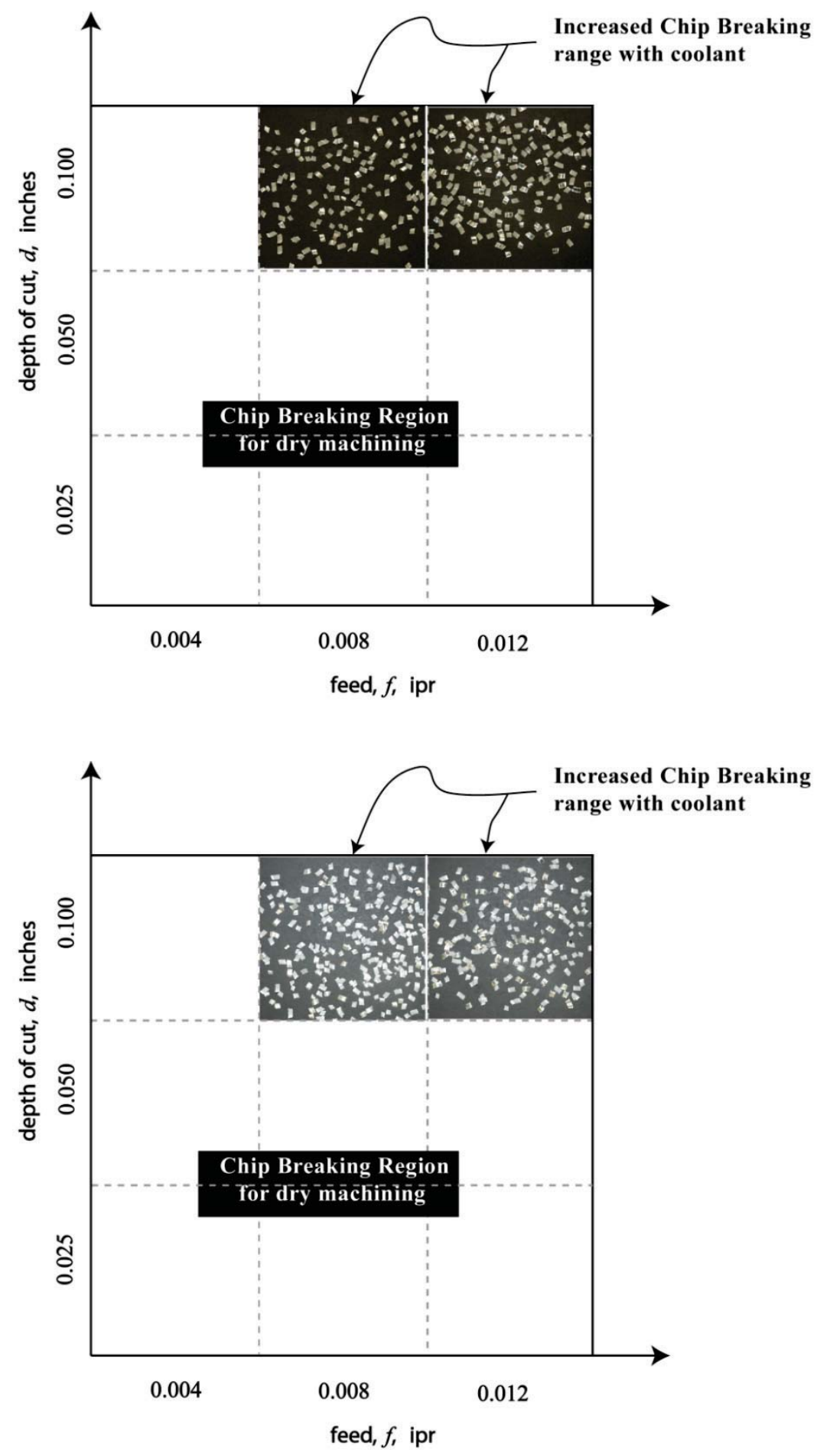

Figure H.2: UCB-R chip breaking tests with coolant (top) Replication \#2 (bottom) Replication \#3. 


\section{APPENDIX I}

\section{COPYRIGHT PERMISSIONS}

The images used in this dissertation appear with permission and courtesy of the individual copyright holders and the publishing entity. Permissions obtained for reproducing these images are listed below for each of the Figures from the dissertation document. No part of any material republished in this dissertation may be copied or reproduced for any purpose without the explicit written permission of the stated copyright holders. Permissions may be obtained for all images either via http://www.copyright.com (Copyright Clearance Center) or by writing to individual copyright holders.

Figure 2.1: (a) Orthogonal cutting model (b) Oblique cutting model (c) General representation of the variables of the cutting process. (Reproduced with permission from Boothroyd and Knight, 2006, (C) 2006 Taylor and Francis Group).

Fundamentals of machining and machine tools Order detail ID: 47645664

ISBN: 978-1-57444-659-3

Publication Year: 2006

Publication Type: Book

Publisher: TAYLOR \& FRANCIS GROUP LLC Rightsholder: TAYLOR \& FRANCIS GROUP LLC - BOOKS

Author/Editor: Boothroyd G and Knight W

A

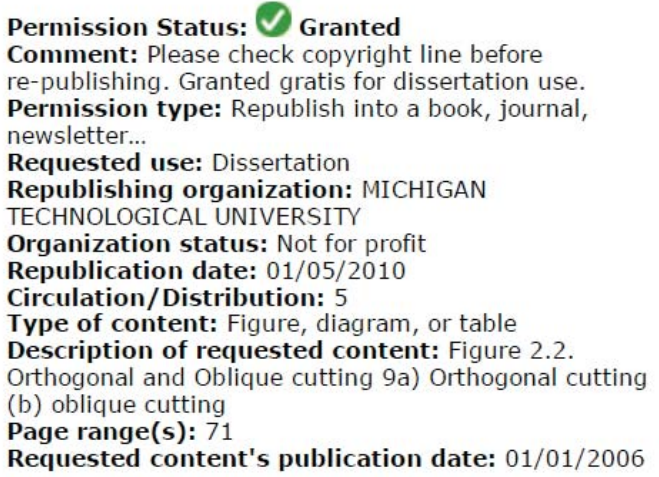

Figure 2.6: Cook's plate analogy for chip formation. (Cook et al., 1954)

Figure 3.1: Finnie's demonstration of chip flow changes with a minor disturbance.

(Finnie, 1963) 
Figure 3.2: Bending moment induced in a chip loaded under tensile and compressive loading. (Nakayama, 1963)

All images reproduced with permission, (C) 1954, 1963, The American Society of Mechanical Engineers.

REVISED: ASME PUBLICATIONS PERMISSION

Friday, November 12, 2010 10:42:06

REQUEST

From: DarchiB@asmestaff.org

To: tgkumber@mtu.edu

Dear Mr. Kumbera Ganapathi:

$\mathrm{i}$ have revised this permission to reflect all requests. It is our pleasure to grant you permission to use the following ASME materials:

- Figure 10 from "Discontinuous Chip Formation," by Cook, N. H., Finnie, I. and Shaw M.C., Transactions of the ASME, Vol. 76, 1954

- Figure 8 from "A comparison of stress strain behavior in cutting with that in other materials tests," by Finnie I, ASME International Research in Production Engineering, 1963

- Figure 6 from "Mechanical properties of chips produced in metal cutting," by Nakayama K, ASME International Research in Production Engineering, 1963

cited in your letter for inclusion in a Doctoral Thesis entitled Feasibility of a Universal Chip Breaking Mechanism to be published by Michigan Technological University.

As is customary, we ask that you ensure full acknowledgment of this material, the author(s), source and ASME as original publisher on all printed copies being distributed.

Figure 2.7: Material flow around the tool tip. (Reproduced with permission, from Connoly and Rubenstien, 1968, (C) Elsevier Science and Technology Journals).

International journal of machine tool design \& research Order detail ID: 47494164

ISSN: 0020-7357

Publication Year: 1968

Publication Type: Journal

Publisher: PERGAMON PRESS.

Permission Status: $\checkmark$ Granted

Rightsholder: ELSEVIER SCIENCE \&

TECHNOLOGY JOURNALS

Author/Editor: Connoly R. and Rubenstein

C.

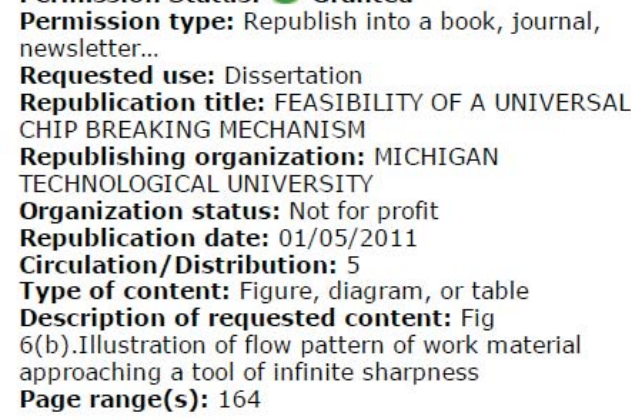

Figure 2.15: Up-curl radius as a function of the contact length. (Reproduced with permission, from van Luttervelt, 1976, (C) 1976 Elsevier Science and Technology Journals). 
ISSN: 0007-8506

Publication Year: 1976

Publication Type: Journal

Publisher: EDITION COLIBRI AG

Rightsholder: ELSEVIER SCIENCE \&

TECHNOLOGY JOURNALS

Author/Editor: vsn Luttervelt C. A
Permission Status: $\mathcal{V}$ Granted

Permission type: Republish into a book, journal,

newsletter...

Requested use: Dissertation

Republishing organization: MICHIGAN

TECHNOLOGICAL UNIVERSITY

Organization status: Not for profit

Republication date: 01/05/2011

Circulation/Distribution: 5

Type of content: Figure, diagram, or table

Description of requested content: Fig 6

Page range(s): 72

Requested content's publication date: 02/25/1976
Billing Status: Not Billed

Figure 2.16: The 3D chip flow (a) Individual components (b) Combined helix form. (Reproduced with permission, from Nakayama and Arai, 1992, (C) $1992 \mathrm{El}$ sevier Science and Technology Journals).

ISSN: $0007-8506$

Publication Year: 1992

Publication Type: Journal

Publisher: EDITION COLIBRI AG

Rightsholder: ELSEVIER SCIENCE \&

TECHNOLOGY JOURNALS

Author/Editor: Nakayama K. and Arai M

\author{
Permission Status: $\mathcal{V}$ Granted \\ Permission type: Republish into a book, journal \\ newsletter.. \\ Requested use: Dissertation \\ Republishing organization: MICHIGAN \\ TECHNOLOGICAL UNIVERSITY \\ Organization status: Not for profit \\ Republication date: 05/01/2011 \\ Circulation/Distribution: 5 \\ Type of content: Figure, diagram, or table \\ Description of requested content: Fig 2. Helix as \\ the compound of two orthogonal circular arcs \\ Page range(s): 72 \\ Requested content's publication date: 01/06/1992
}

Figure 2.17: The concept of total chip control. (Reproduced with permission, from Jawahir et al., 1993, (C) Elsevier Science and Technology Journals).

Rightsholder: ELSEVIER SCIENCE \& TECHNOLOGY JOURNALS

Author/Editor: Jawahir I S and van Luttervelt C A

\author{
Republishing organization: MICHIGAN \\ TECHNOLOGICAL UNIVERSITY \\ Organization status: Not for profit \\ Republication date: 01/05/2011 \\ Circulation/Distribution: 5 \\ Type of content: Figure, diagram, or table \\ Description of requested content: Fig 1 . The total \\ chip control system \\ Page range(s): 661 \\ Requested content's publication date: 01/06/1993
}

Figure 2.18: Work material considerations of chip breakage. (Reproduced with permission, from Jawahir et al., 1993, (C) Elsevier Science and Technology Journals). 
CIRP annals ... manufacturing technology

Order detail ID: 47497164

ISSN: 0007-8506

Publication Year: 1993

Publication Type: Journal

Publisher: EDITION COLIBRI AG

Rightsholder: ELSEVIER SCIENCE \&

TECHNOLOGY JOURNALS

Author/Editor: Jawahir I S and van

Permission Status: $\mathcal{V}$ Granted

Permission type: Republish into a book, journal,

newsletter..

Requested use: Dissertation

Republishing organization: MICHIGAN

TECHNOLOGICAL UNIVERSITY

Organization status: Not for profit

Republication date: 05/01/2011

Circulation/Distribution: 5

Type of content: Figure, diagram, or table

Description of requested content: Fig 37. Work

Material, Shear Angle and tool wear effects on chip

breakability

Page range(s): 680

Requested content's publication date: 01/06/1993
Billing Status: Not Billed

Figure 2.19: Different modes of chip breaking proposed by Nakayama. (Reproduced with permission, from Worthington and Redford, 1973, ( 1973 Elsevier Science and Technology Journals).

International journal of machine tool design \& research Order detail ID: 47966224

ISSN: $0020-7357$

Publication Year: 1973

Publication Type: Journal

Publisher: PERGAMON PRESS.

Rightsholder: ELSEVIER SCIENCE \&

TECHNOLOGY JOURNALS

Author/Editor: Worthington $\mathrm{B}$ and

Redford A H
Permission Status: $\mathcal{V}$ Granted

Permission type: Republish into a book, journal, newsletter..

Requested use: Dissertation

Republishing organization: MICHIGAN

TECHNOLOGICAL UNIVERSITY

Organization status: Not for profit

Republication date: 01/05/2011

Circulation/Distribution: 5

Type of content: Figure, diagram, or table

Description of requested content: Fig 4. Types of

Chip Breaking (after Nakayama)

Page range(s): 261

Requested content's publication date: 06/11/1973
Billing Status: Not Billed

Figure 2.20: Chip breaker action shown for a threading operation.

(Sandvik Coromant, 1996)

Figure 2.21: Chip breaker designs. (Sandvik Coromant, 1996)

Figure 2.22: Chip form as a function of feed and depth of cut. (Sandvik, 1996)

All images reproduced with permission, from Sandvik Coromant, (C) 1996 Sandvik

Coromant. 
Dear Mr. Kumbera:

With this e-mail Sandvik Coromant Company hereby grants you permission to use the images referenced in your request for your intended purpose. Kindly give credit within the body of your work to Sandvik Coromant Company for your use of the images.

With best regards,

Robert Kogan

Team Leader - Creative Services

Communications Dept.

Sandvik Coromant Co.

1702 Nevins Road

Fair Lawn, NJ 07410

Figure 2.23: Kluft's recommendation for acceptable chip forms. (Reproduced with permission, from Kluft et al., 1979, (C) 1979 Elsevier Science and Technology Journals).

CIRP annals ... manufacturing technology

Order detail ID: 47497167

ISSN: 0007-8506

Publication Year: 1979

Publication Type: Journal

Publisher: EDITION COLIBRI AG

Rightsholder: ELSEVIER SCIENCE \&

TECHNOLOGY JOURNALS

Author/Editor: Kluft W, Konig W, van

Luttervelt C A, Nakayama K, Pekelharing A ]
Permission Status: $\checkmark$ Granted

Permission type: Republish into a book, journal, newsletter...

Requested use: Dissertation

Republishing organization: MICHIGAN

TECHNOLOGICAL UNIVERSITY

Organization status: Not for profit

Republication date: $05 / 01 / 2011$

Circulation/Distribution: 5

Type of content: Figure, diagram, or table

Description of requested content: Fig 2. Chip form

classification

Page range(s): 442

Requested content's publication date: 01/06/1979
Billing Status: Not Billed

Figure 2.24: Tabulated chip packing density index. (Reproduced with permission, from Zhang, X. D et al., 1995, (C) 1995 Elsevier Science and Technology Journals ).

Journal of materials processing technology

Order detail ID: 47497170

ISSN: 0924-0136

Publication Year: 1995

Publication Type: Journa

Publisher: ELSEVIER S.A.

Rightsholder: ELSEVIER SCIENCE \&

TECHNOLOGY JOURNALS

Author/Editor: Zhang, X D, Lee, L C and

Seah K W H

Permission Status: $V_{\text {Granted }}$
Permission type: Republish into a book, journal,
newsletter...
Requested use: Dissertation
Republishing organization: MICHIGAN
TECHNOLOGICAL UNIVERSITY
Organization status: Not for profit
Republication date: $01 / 05 / 2011$
Circulation/Distribution: 5
Type of content: Figure, diagram, or table
Description of requested content: Table1. Chip
Packing Density Index
Page range(s): 216
Requested content's publication date: $01 / 05 / 1995$

Figure 3.4: Two possible cases that can result in backward bending. (Reproduced with permission, from Henriksen, 1955, () 1955 Society of Manufacturing Engi-

neers). 
FINDINGS AND DIRECTIONS IN CHIP BREAKERS RESEARCH

Order detail ID: 47494684

OCLC Number: 473710709

Publication Year: 1955

Publication Type: Book

Publisher: SOC OF MANUFACTURING

ENGRS

Rightsholder: SOCIETY OF

MANUFACTURING ENGINEERS (S M E)

Author/Editor: Henriksen E. K.

\author{
Permission Status: $V$ Granted \\ Permission type: Republish into a book, journal, \\ newsletter... \\ Requested use: Dissertation \\ Republishing organization: MICHIGAN \\ TECHNOLOGICAL UNIVERSITY \\ Organization status: Not for profit \\ Republication date: 01/05/2011 \\ Circulation/Distribution: 5 \\ Type of content: Figure, diagram, or table \\ Description of requested content: Disastrous effect \\ of incorrect assembly of clamped chip breaker: (A) \\ start of cut; (B) end of chip trapped under breaker \\ block; (C) chip breaker crowded; (D) chip breaker \\ destroyed. \\ Page range(s): 18
}

Figure 3.5: (a)-(b) Illustration of the backward bending feasiblity tests (c) Picture of mock up tooling. (EMI, 2005)

Figure 3.6: Chips obtained from (left) CNMG432 benchmark insert (right) backward bending tooling. (EMI, 2005)

Figure 3.7: Change in chip forms. (EMI, 2005)

All images reproduced with permission, from EMI LLC, 2005, ( 2005 EMI.

Re: permission for using EMI/Bill Endres copyright $\quad$ Sunday, December 05, 2010 10:18:48 material.

From: wjendres@endresmachining.com

To: tgkumber@mtu.edu

Sender: wjendres@gmail.com

Tim,

On behalf of the copyright holder on both articles noted. Endres Machining Innovations, LLC, by way of this email I grant permission for the use of all images requested.

Regards,

Bill Endres

William J. Endres, Ph.D

President

Endres Machining Innovations, LLC

1402 E. Sharon Ave., Suite 1001

Houghton, MI 49931

www.endresmachining.com

Technology at the cutting edge.

Figure 4.8: Tool-chip contact regions with variation in cutting parameters. (Reproduced with permission, from Nedess and Hintze, 1989, ( 1989 Elsevier Science and Technology Journals). 
CIRP annals ... manufacturing technology

Order detail ID: 47660684

ISSN: 0007-8506

Publication Year: 1989

Publication Type: Journal

Publisher: EDITION COLIBRI AG

Rightsholder: ELSEVIER SCIENCE \&

TECHNOLOGY JOURNALS

Author/Editor: Nedess, C and Hintze W

Permission Status: $\mathcal{V}$ Granted

Permission type: Republish into a book, journal,

newsletter..

Requested use: Dissertation

Republishing organization: MICHIGAN

TECHNOLOGICAL UNIVERSITY

Organization status: Not for profit

Republication date: $01 / 05 / 2010$

Circulation/Distribution: 5

Type of content: Figure, diagram, or table

Description of requested content: Fig 2.

Characteristic parameters of chip control in turning

operations with indexable inserts and three

dimensionally shaped chip formers

Page range(s): 76

Requested content's publication date: 01/01/1989
Billing Status:

Not Billed

Figure 4.10: Deviations of Stabler's rule. (Reproduced with permission, from Brown and Armarego, 1964, ( 1964 Elsevier Science and Technology Journals).

International journal of machine tool design \& research

Order detail ID: 47646165

ISSN: $0020-7357$

Publication Year: 1964

Publication Type: Journal

Publisher: PERGAMON PRESS

Rightsholder: ELSEVIER SCIENCE \&

TECHNOLOGY JOURNALS

Author/Editor: Brown R H and Armarego

E J A

Permission Status: $\checkmark$ Granted

Permission type: Republish into a book, journal,

newsletter..

Requested use: Dissertation

Republishing organization: MICHIGAN

TECHNOLOGICAL UNIVERSITY

Organization status: Not for profit

Republication date: 01/05/2010

Circulation/Distribution: 5

Type of content: Figure, diagram, or table

Description of requested content: Fig 6 . Chip flow

angle from force measurement against agnle fo

obliquity (removing estimated tool edge forces)

Page range(s): 18

Figure 4.11: The equivalent cutting edge for oblique cutting. (Reproduced with permission, from Hu et al., 1986, (C) 1986 Sage Publications Ltd). 
RE: Republication Permission Request Form

From: valerie.bernard@sagepub.co.uk

To: tgkumber@mtu.edu

Dear Thimmaiah Kumbera Ganapathi,

Thank you very much for your request (copied below).

You are very welcome to use the material as per below in your $\mathrm{PhD}$ dissertation.

Please consider this email as written permission and make sure to give full academic referencing to the original material.

Kind regards,

Valérie Bernard

Permissions Assistant

SAGE Publications Ltd

1 Oliver's Yard, 55 City Road

London, EC1Y 1SP

UK

Figure 4.13: Seethaler and Yellowley's numerical model showing deviations of Stabler's rule due to friction. (Reproduced with permission, from Seethaler and Yellowley, 1996, (C) 1996 Elsevier Science and Technology Journals).

International journal of machine tools \& manufacture Order detail ID: 47646164

ISSN: $0890-6955$

Publication Year: 1997

Publication Type: Journal

Publisher: ELSEVIER INC.

Rightsholder: ELSEVIER SCIENCE \&

TECHNOLOGY JOURNALS

Author/Editor: Seethaler R J and

Yellowley I

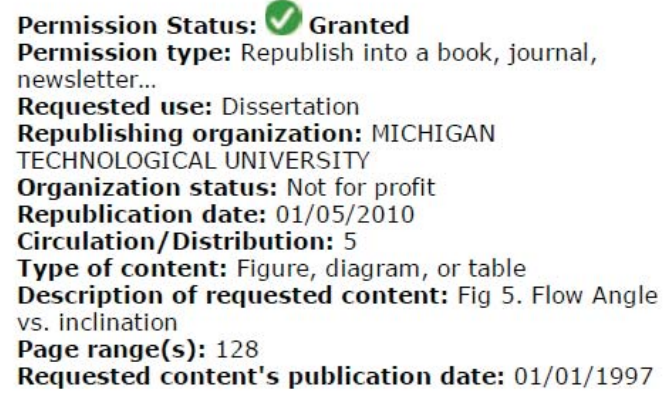

Requested content's publication date: 01/01/1997

Billing Status: Not Billed

Figure 5.1: Chip form classification based on the ISO3685:1993 standard. (Reproduced with permission, from ISO 3685:1993, (C) 1993 ISO/ANSI). 
Dear Thimmaiah,

We are pleased to grant you permission to include Figure G.1, "Chip

Forums" from ISO 3685:1993 in your Ph.D dissertation paper you are

writing. The figure must be cited as follows:

This material is reproduced from Iso 3685:1993 with permission of the

American National Standards Institute (ANSI) on behalf of the

International organization for Standardization (ISO). No part of this

material may be copied or reproduced in any form, electronic retrieval

system or otherwise or made available on the Internet, a public network, by satellite or otherwise without the prior written consent of the ANSI. Copies of this standard may be purchased from the ANSI, 25 West 43rd

Street, New York, NY 10036, (212) 642-4900, http://webstore.ansi.org"

ANSI wishes you success with the completion Ph.D dissertation and in your future endeavors.

Regards,

Tim Dovan

Customer Service Manager

ANSI 